\title{
Exploring psychosis and multidirectional violence
}

Citation for published version (APA):

Honings, S. T. H. (2017). Exploring psychosis and multidirectional violence: a prospective study in the general population. [Doctoral Thesis, Maastricht University]. Datawyse / Universitaire Pers Maastricht. https://doi.org/10.26481/dis.20171222sh

Document status and date:

Published: 01/01/2017

DOI:

10.26481/dis.20171222sh

Document Version:

Publisher's PDF, also known as Version of record

\section{Please check the document version of this publication:}

- A submitted manuscript is the version of the article upon submission and before peer-review. There can be important differences between the submitted version and the official published version of record.

People interested in the research are advised to contact the author for the final version of the publication, or visit the DOI to the publisher's website.

- The final author version and the galley proof are versions of the publication after peer review.

- The final published version features the final layout of the paper including the volume, issue and page numbers.

Link to publication

\footnotetext{
General rights rights.

- You may freely distribute the URL identifying the publication in the public portal. please follow below link for the End User Agreement:

www.umlib.nl/taverne-license

Take down policy

If you believe that this document breaches copyright please contact us at:

repository@maastrichtuniversity.nl

providing details and we will investigate your claim.
}

Copyright and moral rights for the publications made accessible in the public portal are retained by the authors and/or other copyright owners and it is a condition of accessing publications that users recognise and abide by the legal requirements associated with these

- Users may download and print one copy of any publication from the public portal for the purpose of private study or research.

- You may not further distribute the material or use it for any profit-making activity or commercial gain

If the publication is distributed under the terms of Article $25 \mathrm{fa}$ of the Dutch Copyright Act, indicated by the "Taverne" license above, 
(C) copyright Steven Honings, Maastricht 2017

Printing: Datawyse | Universitaire Pers Maastricht

Cover illustration: Glory (2014), (c) copyright Cleon Peterson. Used with permission of the artist.

ISBN 9789461597915 


\title{
Exploring psychosis and multidirectional violence:
}

\section{a prospective study in the general population}

\author{
PROEFSCHRIFT \\ Ter verkrijging van de graad van doctor aan de Universiteit Maastricht, \\ op gezag van de Rector Magnificus, prof. dr. Rianne M. Letschert, \\ volgens het besluit van het College van Decanen, \\ in het openbaar te verdedigen op vrijdag 22 december 2017 om 14.00 uur \\ door
}

Steven Thomas Hubertina Honings 


\section{Promotor:}

Prof. dr. Jim van os

\section{Copromotor:}

Dr. Marjan Drukker

Beoordelingscommissie:

Prof. dr. Ph. Delespaul (voorzitter)

Dr. U. Reininghausen

Prof. dr. J.P.C.J. Selten

Dr. W. Veling (Universitair Medisch Centrum Groningen)

Prof. dr. R.J. Verkes (Radboud Universiteit Nijmegen) 
Paranimfen:

Henri Boersma

Martijn Dremmen 
"Run to the bedroom,

In the suitcase on the left

You'll find my favourite axe.

Don't look so frightened

This is just a passing phase,

One of my bad days.

Would you like to watch T.V.?

Or get between the sheets?

Or contemplate the silent freeway?

Would you like something to eat?

Would you like to learn to fly?

Would you like to see me try?

Would you like to call the cops?

Do you think it's time I stopped?

Why are you running away?"

Roger Waters (1979). One of my turns (Recorded by Pink Floyd). On The Wall. 


\section{Contents}

Chapter 1 General introduction and outline of the thesis

Chapter 2 Psychotic experiences and risk of self-injurious behaviour in the general population: a systematic review and meta-analysis

Chapter 3 Psychotic experiences and incident suicidal ideation and behaviour: disentangling the longitudinal associations from connected psychopathology

Chapter 4 Psychotic experiences and risk of violence perpetration and arrest in the general population: a prospective study

Chapter 5 The interplay of psychosis and victimisation across the life course: a prospective study in the general population

Chapter 6 Discussion

Valorisation

Summary

Samenvatting

Dankwoord

Curriculum vitae

List of publications 

Chapter

General introduction and outline of the thesis 

Psychosis and violence are both prevalent in the general population and are often associated with each other, both in popular media (Owen 2012) and in scientific literature (Fazel et al. 2009). According to the definition of violence by the World Health Organization, different types of violence exist, and violence can be either self-directed or directed towards others (Krug et al. 2002). In other words, violence is a multidirectional phenomenon. In popular media, individuals with psychotic disorder are often depicted as violent, both towards themselves and towards others (Owen 2012). Scientific literature partly supports this common stereotype by showing that individuals with psychotic disorder are at increased risk of violence perpetration (Douglas et al. 2009; Fazel et al. 2009; Large and Nielssen 2011; Nielssen and Large 2010; Reagu et al. 2013) and suicidal behaviour (Hor and Taylor 2010). However, studies show that violence victimisation is prevalent among individuals with psychotic disorder as well (Morgan et al. 2016; de Mooij et al. 2015; Hart et al. 2012; Kamperman et al. 2014), and that individuals with psychotic disorder are more likely to be victims of violence than perpetrators (ten Have et al. 2014; Choe et al. 2008; Brekke et al. 2001). Moreover, although mental disorders are statistically linked to violence perpetration, only $3-5 \%$ of community violence is attributable to mental disorders (Swanson 1994), thus showing that most violence is perpetrated by individuals without psychotic disorder.

Although the link between full-blown psychotic disorder and violence is established in the literature, less is known about the associations between psychotic experiences and multidirectional violence in the general population. Psychotic experiences are hallucinations and delusions below the threshold of a clinical diagnosis of psychotic disorder (Linscott and van Os 2013). There is accumulating evidence that psychotic experiences are part of an extended psychosis phenotype, and thus share demographical, environmental, familial and psychopathological correlates with full-blown psychotic disorder (van Os and Linscott 2012; van Os and Reininghaus 2016). Therefore, it could be hypothesized that analogous to the associations between full-blown psychotic disorder and different types of violence, psychotic experiences are associated with multidirectional violence as well. However, longitudinal studies on the associations between psychotic experiences and violence are scarce.

In the present thesis, the associations between psychotic experiences and multidirectional violence are examined in the general population, using data from three prospective samples: the Early Developmental Stages of Psychopathology study (EDSP) and the first and second Netherlands Mental Health Survey and Incidence Studies (NEMESIS and NEMESIS-2). The thesis starts with a general introduction, providing background information about psychosis and violence, the two main concepts of this thesis. 


\section{A historical overview of psychosis}

In modern psychiatry, the term psychosis is commonly used to refer to hallucinations and delusions. The occurrence of these phenomena is likely to be as old as mankind. However, throughout history, the attitude towards individuals with psychosis has varied greatly. According to Foucault, up until the Renaissance, madness was an experience that was integrated into society, and individuals with hallucinations and delusions were often regarded as individuals possessing some sort of wisdom. However, from the $17^{\text {th }}$ century onwards, driven by the Enlightenment, attitudes towards individuals with hallucinations and delusions shifted and these phenomena started to be regarded as signs of mental illness, requiring social exclusion and diagnostic language to describe the diseases (Foucault 2013).

The word psychosis was first presented in the literature in 1841 by Carl Friedrich Canstatt (1841). In that time period, the word neurosis was used to refer to all diseases of the nervous system, including both psychiatric and neurological disorders (Berrios 1987; Knoff 1970). According to Canstatt, psychosis was a psychic neurosis, in other words, a psychic manifestation of a disease of the nervous system. This broad definition was supported throughout the $19^{\text {th }}$ century by other authors, and psychosis became a synonym for insanity, mental illness and mental disorder (Burgy 2008).

By the end of the $19^{\text {th }}$ and the beginning of the $20^{\text {th }}$ century, advancements were made in the description and specification of the previously heterogeneous concept of psychosis. The German psychiatrist Emil Kraepelin played an important role in this advancement by introducing the concepts dementia praecox and manic-depressive psychosis (Kraepelin 1899), the precursors of what would later become schizophrenia and bipolar disorder. Furthermore, the upcoming of the psycho-analytical movement, which focussed on the psychological rather than the organic origins of mental diseases, caused a conceptual inversion of the term neurosis (Burgy 2008). Whereas the word neurosis was used to refer to all diseases of the nervous system in the $19^{\text {th }}$ century (Berrios 1987), by the beginning of the $20^{\text {th }}$ century, neuroses were defined as mental disorders with psychological or biographical causes. Thus, neuroses were regarded as developments on a continuum with health. Psychoses, on the other hand, were defined as mental disorders resulting from somatic illness and were, therefore, not on a continuum with health (Jaspers 1913). This dichotomy between psychosis and neurosis became the foundation of psychiatric nosology.

The further development of the concept of psychosis throughout the $20^{\text {th }}$ century, is reflected by the definitions of psychosis in de various editions of the Diagnostic and Statistical Manual of Mental Disorders (DSM). In the first edition of the DSM, published in 1952, psychotic disorders were described as disorders "characterized by a varying degree of personality disintegration and failure to test and evaluate correctly external reality in various spheres" (American Psychiatric Association 1952). Therefore, in the DSM-I, psychosis was considered a disorder affecting the complete personality of the 
person. In the DSM-II, the definition of psychosis was broadened, and patients were described as psychotic when "their mental functioning is sufficiently impaired to interfere grossly with their capacity to meet the ordinary demands of life" (American Psychiatric Association 1968). In this definition, the focus is on the severity of functional impairment. Hallucinations and delusions may cause this functional impairment, but other causes are possible as well.

The dichotomy between psychosis and neurosis persisted throughout DSM-I and DSM-II. However, from DSM-III onwards, this dichotomy was discarded, and etiological concepts were replaced by descriptive criteria based on empirical evidence (Bayer and Spitzer 1985). In DSM-III, the definition of psychosis focussed on the presence of certain symptoms, rather than on personality disintegration or functional impairment. More specifically, the word psychosis was omitted and replaced by the adjectival form psychotic, defined as: "gross impairment in reality testing" (American Psychiatric Association 1980). Psychotic symptoms could be present in both psychotic and nonpsychotic mental disorders. This trend was continued in the DSM-IV, in which the term 'psychotic' referred to the presence of delusions and hallucinations (American Psychiatric Association 2000).

Further advancements were made in the DSM-5, in which a dimensional approach for psychosis was introduced (American Psychiatric Association 2013). In this approach, psychosis is no longer conceptualized as a dichotomous entity, but psychotic symptoms are considered to be on a continuum with normal mental states (Allardyce et al. 2007). This new conceptualization of psychosis reflected the results of numerous studies in recent years, that showed that psychotic symptoms were not only present in individuals with psychotic disorders, but were also prevalent among individuals in the general population (van Os et al. 2009). These findings have led to the suggestion of an extended psychosis phenotype (van Os and Linscott 2012).

\section{The extended psychosis phenotype}

The historical overview of the definition of psychosis presented in the previous paragraph shows that since the first introduction of the concept of psychosis, the definition has gradually shifted. During a large part of the $20^{\text {th }}$ century, psychosis was considered a severe mental disorder with impaired reality testing, leading to severe, functional impairment. Moreover, psychosis was considered as a discrete disease, probably of organic origin, which was, therefore, not on a continuum with health. In modern psychiatry, the definition of schizophrenia, the prototype of psychosis, written by the National Institute of Mental Health (NIMH), still reflects this old conceptualization of psychosis: "Schizophrenia is a chronic and severe mental disorder that affects how a person thinks, feels, and behaves. People with schizophrenia may seem like they have lost touch with reality. Although schizophrenia is not as common as other mental disorders, the symp- 
toms can be very disabling" (National Institute of Mental Health 2017). The prevalence of schizophrenia is approximately $1 \%$ in the general population; the prevalence of broadly defined psychotic disorder is 3.5\% (Perala et al. 2007). However, hallucinations and delusions, the clinical hallmarks of schizophrenia, are not exclusive to schizophrenia or other psychotic disorders. Already in 1969, Strauss reported that hallucinations and delusions were prevalent in clinical, non-psychotic patients. In addition, he suggested an alternative to the at that time common conceptualization of psychosis as a discrete, discontinuous concept. According to Strauss (1969), the evaluation of patients with psychosis could be improved by "conceptualizing hallucinations and delusions as points on continua, rather than discontinuous entities", because he thought that the conceptualization of psychosis as a discontinuous concept led to the conception that individuals with psychosis were qualitatively different from people without psychosis. However, only in the past two decades, studies started examining this alternative conceptualization of psychosis.

In the literature, hallucinations and delusions falling under the threshold of a diagnosable psychotic disorder are often labelled as psychotic experiences. A meta-analysis has shown that the prevalence of psychotic experiences is $7.2 \%$ in the adult, general population (Linscott and van Os 2013). In children and adolescents, the prevalence is even higher (Kelleher et al. 2012a). Thus, the prevalence of psychotic experiences is much higher than the population prevalence of broadly defined psychotic disorder. This finding has led to the suggestion of an extended psychosis phenotype in the general population, i.e. a phenotype ranging from psychotic experiences on the one hand, to full-blown psychosis on the other, while sharing demographical, environmental, familial and psychopathological correlates (van Os and Linscott 2012; van Os and Reininghaus 2016; van Os et al. 2000; van Os et al. 2009). Psychotic experiences are often transitory. It has been shown that psychotic experiences persist in approximately $20 \%$ of individuals with psychotic experiences, while $80 \%$ remits over time; $7 \%$ of the individuals with baseline psychotic experiences continues to develop a psychotic disorder (Linscott and van Os 2013). Therefore, schizophrenia and other psychotic disorders have been conceptualized as the poor outcome of a common phenotype characterized by the persistence of psychotic experiences (Dominguez et al. 2011), while psychotic experiences likely represent the softest behavioural expression of distributed population risk of psychosis (Lataster et al. 2009; van Nierop et al. 2012; van Os and Reininghaus 2016).

Evidence suggests that psychotic experiences are not only phenotypically continuous with psychotic disorder, but also aetiologically. Non-genetic risk factors associated with schizophrenia and other psychotic disorders, including exposure to cannabis, urbanicity and traumatic experiences, have been similarly associated with psychotic experiences (van Os et al. 2009). Moreover, reasoning biases that are common in psychotic disorders, for example the tendency to jump to conclusions, have been associated with psychotic experiences as well (Bentall et al. 2009; Broome et al. 2007; Freeman et al. 2008). Furthermore, studies suggest that aberrant salience, which is suggested to play 
an important role in schizophrenia (Kapur 2003), is also present in individuals with psychotic experiences (Galdos et al. 2011). Neurocognitive alterations which are common in psychotic disorder, for example reduced processing speed and working memory, are also more prevalent in individuals with psychotic experiences than in those without these experiences (Barnett et al. 2012; Blanchard et al. 2010; Cullen et al. 2010; Kelleher et al. 2013a; Kelleher et al. 2015). Thus, evidence suggests an aetiological continuity between psychotic experiences and psychotic disorder

Most studies until now focussed on the subclinical experience of positive psychotic symptoms (i.e. delusions and hallucinations) when studying the extended psychosis phenotype, although negative and disorganized symptoms are also prevalent in schizophrenia and other psychotic disorders. However, available evidence shows that subclinical experiences of negative and disorganized symptoms are as prevalent as subclinical experiences of positive symptoms in the general population. Moreover, the cooccurrence of positive symptoms with negative and disorganized symptoms in individuals without psychotic disorder was associated with an increased risk of future psychotic disorder, functional impairment and help-seeking behaviour (Dominguez et al. 2010; Werbeloff et al. 2015). Thus, there is evidence that both the positive and the negative symptom dimensions of psychosis are present in the suggested extended psychosis phenotype.

Recently, researchers suggested that psychotic experiences represent not only a specific extended psychosis phenotype, but also a transdiagnostic, extended phenotype (van Os and Reininghaus 2016). Studies show that the majority of the individuals with psychotic experiences have at least one non-psychotic mental disorder, and that individuals with psychotic experiences in absence of a non-psychotic mental disorder are at increased risk to develop one (DeVylder et al. 2014; Kelleher et al. 2012b). Moreover, the presence of psychotic experiences in individuals with affective and anxiety disorders has been associated with poorer treatment response and greater levels of nonpsychotic illness severity (Kelleher et al. 2012b). Therefore, psychotic experiences have been identified as an indicator of severity in non-psychotic psychopathology (Wigman et al. 2012; Wigman et al. 2014; Perlis et al. 2011; Guloksuz et al. 2015; Kelleher et al. 2012b). These findings suggest that psychosis can be conceptualized as a transdiagnostic symptom dimension. However, studies on psychosis as a transdiagnostic dimension are scarce, and more research is required in order to identify transdiagnostically shared environmental and genetic factors (van Os and Reininghaus 2016).

In conclusion, this section has shown that the concept of psychosis has not only changed during the $19^{\text {th }}$ and $20^{\text {th }}$ century, but also in recent years. Since the first introduction of the word psychosis in 1841, the psychosis concept has developed from a broad, heterogeneous group including almost all severe mental disorders in the $19^{\text {th }}$ century, to a complex, transdiagnostic, extended psychosis phenotype. There is now accumulating evidence that psychotic experiences are phenotypically, temporally and 
aetiologically continuous with psychotic disorder. Therefore, psychotic experience can be used to study mechanisms of psychosis in the general population.

\section{Multidirectional violence and psychosis}

Violence is a broad concept that is defined by the World Health Organization as "the intentional use of physical force or power, threatened or actual, against oneself, another person, or against a group or community, that either results in or has a high likelihood of resulting in injury, death, psychological harm, maldevelopment, or deprivation" (Krug et al. 2002). According to this definition, violence can be multidirectional, i.e. it can be self-directed or directed towards others. In this paragraph, background information about self-directed violence, interpersonal violence perpetration and interpersonal violence victimisation is given. Furthermore, an overview of the literature about psychosis and multidirectional violence is provided.

\section{Self-directed violence}

Self-directed violence comprises various forms of self-injurious behaviour, that can be divided into two groups based on the presence or absence of suicidal intent.

Self-injurious behaviour with suicidal intent is usually referred to as suicidality and includes suicidal ideation (i.e. having thoughts about killing oneself), suicidal plans (i.e. considering a specific method through which one intends to die), suicide attempts (i.e. engagement in potentially self-injurious behaviour in which there is some intent to die) and completed suicides (Sveticic and De Leo 2012; Nock 2010), with twelve months prevalences ranging from $3.3 \%$ for suicidal ideation to $0.01 \%$ for completed suicide (Kessler et al. 2005). Every year, more than 800.000 individuals commit suicide and more than 16 million individuals attempt suicide, thus making suicide a major global health burden and the fifteenth leading cause of death worldwide in 2012. In individuals aged fifteen to twenty-nine years old, suicide is second leading cause of death (World Health Organization 2014). Mental disorders are an important risk factor for suicide. Evidence shows that a mental disorder is present in $91 \%$ of the individuals who commit suicide, most often an affective disorder (Cavanagh et al. 2003). The link between schizophrenia and suicide is well-established in the literature as well (Hor and Taylor 2010). However, the association between psychotic experiences and suicidal ideation and behaviour is less clear. Various cross-sectional and longitudinal studies examined the association between psychotic experiences and these outcomes, but results were contradictory (Capra et al. 2015; DeVylder et al. 2015; Jang et al. 2014; Kelleher et al. 2012c; Nishida et al. 2010; Saha et al. 2011; Temmingh et al. 2011; Fisher et al. 2013; Kelleher et al. 2013b; Sharifi et al. 2015; Martin et al. 2015; Sullivan et al. 2015). Moreo- 
ver, studies suffered from various methodological limitations, thus making it difficult to interpret the results of these studies. Therefore, more research is required in order to gain insight into the association between psychotic experiences and suicidal ideation and suicidal behaviour.

In addition to self-directed violence with suicidal intent, forms of self-directed violence without suicidal intent exist as well. In the literature, there is a great variety of definitions and terminology used to refer to self-injurious behaviour without suicidal intent, including parasuicide, self-mutilation and deliberate self-harm (Nock 2010). In the DSM-5, non-suicidal self-injury has been proposed as a new diagnosis for selfinjurious behaviour without suicidal intent (American Psychiatric Association 2013). Non-suicidal self-injury has been identified as a risk factor for suicidal ideation and behaviour (Hawton et al. 2012; Hawton and van Heeringen 2009). However, it is unclear whether self-directed violence with and without suicidal intent are part of the same continuum, as suggested by some authors (Hamza et al. 2012). Similar to suicidality, mental disorders are prevalent among individuals with non-suicidal self-injurious behaviour, including depressive disorder (41.6\%), substance use disorder (59.6\%) and borderline personality disorder (51.7\%) (Nock et al. 2006). The prevalence of non-suicidal selfinjury among individuals with psychotic disorder is unknown. However, psychotic experiences have been associated with non-suicidal self-injury (Koyanagi et al. 2015; Martin et al. 2015), although results were contradictory.

\section{Interpersonal violence}

According to the World Report on Violence and Health, published by the World Health Organisation, interpersonal violence can be directed towards family or towards community. Interpersonal violence is subdivided into four different categories of violence based on the nature of the violent act, namely physical violence, sexual violence, psychological violence and deprivation or neglect (Krug et al. 2002). Since interpersonal violence involves at least two individuals, it can be studied from two perspectives, i.e. the perspective of the perpetrator and the perspective of the victim of the violence.

Violence victimisation may occur in childhood or during adulthood. Evidence shows that interpersonal violence victimisation is widespread. According to a large, epidemiological survey, the prevalence of physical abuse during childhood is $8 \%$. Moreover, $1.6 \%$ of the children in the survey had experiences of sexual abuse (Kessler et al. 2010). Childhood trauma (i.e. victimisation during childhood) has been associated with various mental disorders (Kessler et al. 2010), including depression (Nanni et al. 2012), social anxiety (Kuo et al. 2011) and bipolar disorder (Etain et al. 2008). In addition, childhood trauma has been associated with an increased risk of both psychotic experiences (Kelleher et al. 2013c) and full-blown psychosis (Varese et al. 2012), thus covering the complete extended psychosis phenotype. 
Violence victimisation during adulthood is prevalent as well: $35 \%$ of the women worldwide have experienced physical or sexual violence, most often perpetrated by an intimate partner (World Health Organization 2013). Similar to childhood trauma, adult victimisation has been associated with an increased risk of various mental disorders, including depression, anxiety disorders and suicidal ideation (Palm et al. 2016; Lagdon et al. 2014). Recent studies have shown that victimisation is highly prevalent among individuals with severe mental disorders, including psychotic disorder (de Mooij et al. 2015; Hart et al. 2012; Kamperman et al. 2014). In individuals with psychosis and other severe mental illness, adult victimisation has been associated with several adverse consequences, including impaired community and psychosocial functioning (Hodgins et al. 2009; Lysaker et al. 2001), increased symptom severity, illness chronicity (Newman et al. 2010) and more service use (Mueser et al. 2002). However, it is unclear whether psychosis increases the risk of victimisation, or whether victimisation increases the risk of psychosis, since most studies to date used cross-sectional study designs, thus inferring causal inferences (de Mooij et al. 2015; Hart et al. 2012; Kamperman et al. 2014). Therefore, longitudinal research is required in order to gain insight in the nature of the mechanism between psychosis and victimisation.

The prevalence of interpersonal violence perpetration appears to be lower than the prevalence of violence victimisation. In the general population, the lifetime prevalence of physical and psychological violence perpetration against an intimate partner was $5.9 \%$ and $24.3 \%$, respectively. The three-year prevalence of physical and psychological violence perpetration against any person in general was $2.4 \%$ and $9.2 \%$, respectively (ten Have et al. 2014). Various mental disorders, including psychotic disorder (Coid et al. 2016; Douglas et al. 2009; Fazel et al. 2009; Fazel and Yu 2011; Large and Nielssen 2008; Large et al. 2009; Large and Nielssen 2011; Nielssen and Large 2010; Reagu et al. 2013), bipolar disorder (Fazel et al. 2010), personality disorders (Yu et al. 2012; Kennealy et al. 2010; Hanson and Morton-Bourgon 2005; Andrews et al. 2012), depression (Fazel et al. 2015) and substance abuse (Dowden and Brown 2002; Andrews et al. 2012) have been associated with an increased risk of violence perpetration and arrest. Although numerous studies and meta-analyses examined the association between full-blown psychotic disorders and violence perpetration, few studies examined the association between psychotic experiences and violence perpetration. Available studies show that psychotic experiences are associated with interpersonal violence perpetration and arrest (Kinoshita et al. 2011; Mojtabai 2006; Swanson et al. 1996; Nederlof et al. 2012; Coid et al. 2016). However, as the association between psychotic experiences and violence perpetration has only been examined in cross-sectional studies, longitudinal research is required to investigate these associations further and determine the temporal sequence. 


\section{Aims and outline of the thesis}

In this thesis, longitudinal associations between psychosis and multidirectional violence are examined in the general population. The aim of the thesis is to gain insight into the associations between psychosis and self-directed violence, interpersonal violence perpetration and violence victimisation, and to study whether associations between psychotic experiences and multidirectional violence are analogous to the associations between full-blown psychosis and multidirectional violence.

The association between psychosis and self-directed violence is examined in chapters two and three. In chapter 2, a systematic review and meta-analysis of the studies on psychotic experiences and self-injurious behaviour is presented in order to summarize available evidence and to identify directions for further research. In chapter 3 , the associations between psychotic experiences on the one hand and suicidal ideation and suicidal behaviour on the other are further examined in three prospective general population samples, while addressing methodological limitations identified in previously published studies on these associations.

The associations between psychosis and interpersonal violence are examined in chapters four and five. Chapter 4 reports on the association between psychotic experiences and interpersonal violence perpetration in the general population. More specifically, the association between both self-reported and clinically validated psychotic experiences and interpersonal violence perpetration are examined. Violence perpetration included physical violence towards an intimate partner, psychological violence towards an intimate partner, and arrest. In chapter 5, the association between psychosis and violence victimisation is examined. More specifically, the bidirectional associations between psychotic experiences and adult victimisation are examined, while simultaneously assessing the moderating effect of childhood victimisation.

Finally, in chapter 6 , results from previous chapters are summarized and discussed in a broader perspective. In addition, directions for future research are given. 


\section{References}

Allardyce, J., Gaebel, W., Zielasek, J. \& Van Os, J. 2007. Deconstructing Psychosis conference February 2006: the validity of schizophrenia and alternative approaches to the classification of psychosis. Schizophr Bull, 33, 863-7.

American Psychiatric Association 1952. Diagnostic and statistical manual of mental disorders, Washington, DC, American Psychiatric Association.

American Psychiatric Association 1968. Diagnostic and statistical manual of mental disorders, Washington, DC, American Psychiatric Association.

American Psychiatric Association 1980. Diagnostic and statistical manual of mental disorders, Washington, DC, American Psychiatric Association.

American Psychiatric Association 2000. Diagnostic and statistical manual of mental disorders, Washington, DC, American Psychiatric Association.

American Psychiatric Association 2013. Diagnostic and statistical manual of mental disorders, Washington, DC, American Psychiatric Association.

Andrews, D. A., Guzzo, L., Raynor, P., Rowe, R. C., Rettinger, L. J., Brews, A. \& Wormith, J. S. 2012. Are the major risk/need factors predictive of both female and male reoffending?: a test with the eight domains of the level of service/case management inventory. Int J Offender Ther Comp Criminol, 56, 113-33.

Barnett, J. H., Mcdougall, F., Xu, M. K., Croudace, T. J., Richards, M. \& Jones, P. B. 2012. Childhood cognitive function and adult psychopathology: associations with psychotic and non-psychotic symptoms in the general population. Br J Psychiatry, 201, 124-30.

Bayer, R. \& Spitzer, R. L. 1985. Neurosis, psychodynamics, and DSM-III. A history of the controversy. Arch Gen Psychiatry, 42, 187-96.

Bentall, R. P., Rowse, G., Shryane, N., Kinderman, P., Howard, R., Blackwood, N., Moore, R. \& Corcoran, R. 2009. The cognitive and affective structure of paranoid delusions: a transdiagnostic investigation of patients with schizophrenia spectrum disorders and depression. Arch Gen Psychiatry, 66, 236-47.

Berrios, G. E. 1987. Historical aspects of psychoses: 19th century issues. Br Med Bull, 43, 484-98.

Blanchard, M. M., Jacobson, S., Clarke, M. C., Connor, D., Kelleher, I., Garavan, H., Harley, M. \& Cannon, M. 2010. Language, motor and speed of processing deficits in adolescents with subclinical psychotic symptoms. Schizophr Res, 123, 71-6.

Brekke, J. S., Prindle, C., Bae, S. W. \& Long, J. D. 2001. Risks for individuals with schizophrenia who are living in the community. Psychiatr Serv, 52, 1358-66.

Broome, M. R., Johns, L. C., Valli, I., Woolley, J. B., Tabraham, P., Brett, C., Valmaggia, L., Peters, E., Garety, P. A. \& Mcguire, P. K. 2007. Delusion formation and reasoning biases in those at clinical high risk for psychosis. Br J Psychiatry Suppl, 51, s38-42.

Burgy, M. 2008. The concept of psychosis: historical and phenomenological aspects. Schizophr Bull, 34, 120010.

Canstatt, C. 1841. Handbuch der Medizinischen Klinik, Stuttgart, Enke.

Capra, C., Kavanagh, D. J., Hides, L. \& Scott, J. G. 2015. Subtypes of psychotic-like experiences are differentially associated with suicidal ideation, plans and attempts in young adults. Psychiatry Res, 228, 894-8.

Cavanagh, J. T., Carson, A. J., Sharpe, M. \& Lawrie, S. M. 2003. Psychological autopsy studies of suicide: a systematic review. Psychol Med, 33, 395-405.

Choe, J. Y., Teplin, L. A. \& Abram, K. M. 2008. Perpetration of violence, violent victimization, and severe mental illness: balancing public health concerns. Psychiatr Serv, 59, 153-64.

Coid, J. W., Ullrich, S., Bebbington, P., Fazel, S. \& Keers, R. 2016. Paranoid Ideation and Violence: Metaanalysis of Individual Subject Data of 7 Population Surveys. Schizophr Bull, 42, 907-15.

Cullen, A. E., Dickson, H., West, S. A., Morris, R. G., Mould, G. L., Hodgins, S., Murray, R. M. \& Laurens, K. R. 2010. Neurocognitive performance in children aged 9-12 years who present putative antecedents of schizophrenia. Schizophr Res, 121, 15-23. 
De Mooij, L. D., Kikkert, M., Lommerse, N. M., Peen, J., Meijwaard, S. C., Theunissen, J., Duurkoop, P. W., Goudriaan, A. E., Van, H. L., Beekman, A. T. \& Dekker, J. J. 2015. Victimisation in adults with severe mental illness: prevalence and risk factors. Br J Psychiatry, 207, 515-22.

Devylder, J. E., Burnette, D. \& Yang, L. H. 2014. Co-occurrence of psychotic experiences and common mental health conditions across four racially and ethnically diverse population samples. Psychol Med, 44, 350313.

Devylder, J. E., Lukens, E. P., Link, B. G. \& Lieberman, J. A. 2015. Suicidal ideation and suicide attempts among adults with psychotic experiences: data from the Collaborative Psychiatric Epidemiology Surveys. JAMA Psychiatry, 72, 219-25.

Dominguez, M. D., Saka, M. C., Lieb, R., Wittchen, H. U. \& Van Os, J. 2010. Early expression of negative/disorganized symptoms predicting psychotic experiences and subsequent clinical psychosis: a 10year study. Am J Psychiatry, 167, 1075-82.

Dominguez, M. D., Wichers, M., Lieb, R., Wittchen, H. U. \& Van Os, J. 2011. Evidence that onset of clinical psychosis is an outcome of progressively more persistent subclinical psychotic experiences: an 8-year cohort study. Schizophr Bull, 37, 84-93.

Douglas, K. S., Guy, L. S. \& Hart, S. D. 2009. Psychosis as a risk factor for violence to others: a meta-analysis. Psychol Bull, 135, 679-706.

Dowden, C. \& Brown, S. L. 2002. The role of substance abuse factors in predicting recidivism: A meta-analysis. Psychology Crime \& Law, 8, 243-264.

Etain, B., Henry, C., Bellivier, F., Mathieu, F. \& Leboyer, M. 2008. Beyond genetics: childhood affective trauma in bipolar disorder. Bipolar Disord, 10, 867-76.

Fazel, S., Gulati, G., Linsell, L., Geddes, J. R. \& Grann, M. 2009. Schizophrenia and violence: systematic review and meta-analysis. PLoS Med, 6, e1000120.

Fazel, S., Lichtenstein, P., Grann, M., Goodwin, G. M. \& Langstrom, N. 2010. Bipolar disorder and violent crime: new evidence from population-based longitudinal studies and systematic review. Arch Gen Psychiatry, 67, 931-8.

Fazel, S. \& Yu, R. 2011. Psychotic disorders and repeat offending: systematic review and meta-analysis. Schizophr Bull, 37, 800-10.

Fazel, S., Wolf, A., Chang, Z., Larsson, H., Goodwin, G. M. \& Lichtenstein, P. 2015. Depression and violence: a Swedish population study. Lancet Psychiatry, 2, 224-32.

Fisher, H. L., Caspi, A., Poulton, R., Meier, M. H., Houts, R., Harrington, H., Arseneault, L. \& Moffitt, T. E. 2013. Specificity of childhood psychotic symptoms for predicting schizophrenia by 38 years of age: a birth cohort study. Psychol Med, 43, 2077-86.

Foucault, M. 2013. History of Madness, Taylor \& Francis.

Freeman, D., Pugh, K., Antley, A., Slater, M., Bebbington, P., Gittins, M., Dunn, G., Kuipers, E., Fowler, D. \& Garety, P. 2008. Virtual reality study of paranoid thinking in the general population. Br J Psychiatry, 192, 258-63.

Galdos, M., Simons, C., Fernandez-Rivas, A., Wichers, M., Peralta, C., Lataster, T., Amer, G., Myin-Germeys, I., Allardyce, J., Gonzalez-Torres, M. A. \& Van Os, J. 2011. Affectively salient meaning in random noise: a task sensitive to psychosis liability. Schizophr Bull, 37, 1179-86.

Guloksuz, S., Van Nierop, M., Lieb, R., Van Winkel, R., Wittchen, H. U. \& Van Os, J. 2015. Evidence that the presence of psychosis in non-psychotic disorder is environment-dependent and mediated by severity of non-psychotic psychopathology. Psychol Med, 45, 2389-401.

Hamza, C. A., Stewart, S. L. \& Willoughby, T. 2012. Examining the link between nonsuicidal self-injury and suicidal behavior: a review of the literature and an integrated model. Clin Psychol Rev, 32, 482-95.

Hanson, R. K. \& Morton-Bourgon, K. E. 2005. The characteristics of persistent sexual offenders: a metaanalysis of recidivism studies. J Consult Clin Psychol, 73, 1154-63.

Hart, C., De Vet, R., Moran, P., Hatch, S. L. \& Dean, K. 2012. A UK population-based study of the relationship between mental disorder and victimisation. Soc Psychiatry Psychiatr Epidemiol, 47, 1581-90.

Hawton, K. \& Van Heeringen, K. 2009. Suicide. Lancet, 373, 1372-81. 


\section{Chapter 1}

Hawton, K., Saunders, K. E. \& O'connor, R. C. 2012. Self-harm and suicide in adolescents. Lancet, 379, 237382.

Hodgins, S., Lincoln, T. \& Mak, T. 2009. Experiences of victimisation and depression are associated with community functioning among men with schizophrenia. Soc Psychiatry Psychiatr Epidemiol, 44, 448-57.

Hor, K. \& Taylor, M. 2010. Suicide and schizophrenia: a systematic review of rates and risk factors. J Psychopharmacol, 24, 81-90.

Jang, J. H., Lee, Y. J., Cho, S. J., Cho, I. H., Shin, N. Y. \& Kim, S. J. 2014. Psychotic-like experiences and their relationship to suicidal ideation in adolescents. Psychiatry Res, 215, 641-5.

Jaspers, K. 1913. Allgemeine Psychopathologie Berlin, Springer.

Kamperman, A. M., Henrichs, J., Bogaerts, S., Lesaffre, E. M., Wierdsma, A. I., Ghauharali, R. R., Swildens, W., Nijssen, Y., Van Der Gaag, M., Theunissen, J. R., Delespaul, P. A., Van Weeghel, J., Van Busschbach, J. T., Kroon, H., Teplin, L. A., Van De Mheen, D. \& Mulder, C. L. 2014. Criminal victimisation in people with severe mental illness: a multi-site prevalence and incidence survey in the Netherlands. PLoS One, 9, e91029.

Kapur, S. 2003. Psychosis as a state of aberrant salience: a framework linking biology, phenomenology, and pharmacology in schizophrenia. Am J Psychiatry, 160, 13-23.

Kelleher, I., Connor, D., Clarke, M. C., Devlin, N., Harley, M. \& Cannon, M. 2012a. Prevalence of psychotic symptoms in childhood and adolescence: a systematic review and meta-analysis of population-based studies. Psychol Med, 42, 1857-63.

Kelleher, I., Keeley, H., Corcoran, P., Lynch, F., Fitzpatrick, C., Devlin, N., Molloy, C., Roddy, S., Clarke, M. C., Harley, M., Arseneault, L., Wasserman, C., Carli, V., Sarchiapone, M., Hoven, C., Wasserman, D. \& Cannon, M. 2012b. Clinicopathological significance of psychotic experiences in non-psychotic young people: evidence from four population-based studies. Br J Psychiatry, 201, 26-32.

Kelleher, I., Lynch, F., Harley, M., Molloy, C., Roddy, S., Fitzpatrick, C. \& Cannon, M. 2012c. Psychotic symptoms in adolescence index risk for suicidal behavior: findings from 2 population-based case-control clinical interview studies. Arch Gen Psychiatry, 69, 1277-83.

Kelleher, I., Clarke, M. C., Rawdon, C., Murphy, J. \& Cannon, M. 2013a. Neurocognition in the extended psychosis phenotype: performance of a community sample of adolescents with psychotic symptoms on the MATRICS neurocognitive battery. Schizophr Bull, 39, 1018-26.

Kelleher, I., Corcoran, P., Keeley, H., Wigman, J. T., Devlin, N., Ramsay, H., Wasserman, C., Carli, V., Sarchiapone, M., Hoven, C., Wasserman, D. \& Cannon, M. 2013b. Psychotic symptoms and population risk for suicide attempt: a prospective cohort study. JAMA Psychiatry, 70, 940-8.

Kelleher, I., Keeley, H., Corcoran, P., Ramsay, H., Wasserman, C., Carli, V., Sarchiapone, M., Hoven, C., Wasserman, D. \& Cannon, M. 2013c. Childhood trauma and psychosis in a prospective cohort study: cause, effect, and directionality. Am J Psychiatry, 170, 734-41.

Kelleher, I., Wigman, J. T., Harley, M., O'hanlon, E., Coughlan, H., Rawdon, C., Murphy, J., Power, E., Higgins, N. M. \& Cannon, M. 2015. Psychotic experiences in the population: Association with functioning and mental distress. Schizophr Res, 165, 9-14.

Kennealy, P. J., Skeem, J. L., Walters, G. D. \& Camp, J. 2010. Do core interpersonal and affective traits of PCL-R psychopathy interact with antisocial behavior and disinhibition to predict violence? Psychol Assess, 22, 569-80.

Kessler, R. C., Berglund, P., Borges, G., Nock, M. \& Wang, P. S. 2005. Trends in suicide ideation, plans, gestures, and attempts in the United States, 1990-1992 to 2001-2003. JAMA, 293, 2487-95.

Kessler, R. C., Mclaughlin, K. A., Green, J. G., Gruber, M. J., Sampson, N. A., Zaslavsky, A. M., Aguilar-Gaxiola, S., Alhamzawi, A. O., Alonso, J., Angermeyer, M., Benjet, C., Bromet, E., Chatterji, S., De Girolamo, G., Demyttenaere, K., Fayyad, J., Florescu, S., Gal, G., Gureje, O., Haro, J. M., Hu, C. Y., Karam, E. G., Kawakami, N., Lee, S., Lepine, J. P., Ormel, J., Posada-Villa, J., Sagar, R., Tsang, A., Ustun, T. B., Vassilev, S., Viana, M. C. \& Williams, D. R. 2010. Childhood adversities and adult psychopathology in the WHO World Mental Health Surveys. Br J Psychiatry, 197, 378-85.

Kinoshita, Y., Shimodera, S., Nishida, A., Kinoshita, K., Watanabe, N., Oshima, N., Akechi, T., Sasaki, T., Inoue, S., Furukawa, T. A. \& Okazaki, Y. 2011. Psychotic-like experiences are associated with violent behavior in adolescents. Schizophr Res, 126, 245-51. 
Knoff, W. F. 1970. A history of the concept of neurosis, with a memoir of William Cullen. Am J Psychiatry, 127, 80-4.

Koyanagi, A., Stickley, A. \& Haro, J. M. 2015. Subclinical psychosis and suicidal behavior in England: Findings from the 2007 Adult Psychiatric Morbidity Survey. Schizophr Res, 168, 62-7.

Kraepelin, E. 1899. Psychiatrie. Ein Lehrbuch für Studierende und Aerzte, Leipzig, Barth.

Krug, E. G., Mercy, J. A., Dahlberg, L. L. \& Zwi, A. B. 2002. The world report on violence and health. Lancet, 360, 1083-8.

Kuo, J. R., Goldin, P. R., Werner, K., Heimberg, R. G. \& Gross, J. J. 2011. Childhood trauma and current psychological functioning in adults with social anxiety disorder. J Anxiety Disord, 25, 467-73.

Lagdon, S., Armour, C. \& Stringer, M. 2014. Adult experience of mental health outcomes as a result of intimate partner violence victimisation: a systematic review. Eur J Psychotraumatol, 5.

Large, M. \& Nielssen, O. 2008. Evidence for a relationship between the duration of untreated psychosis and the proportion of psychotic homicides prior to treatment. Soc Psychiatry Psychiatr Epidemiol, 43, 37-44.

Large, M., Smith, G. \& Nielssen, O. 2009. The relationship between the rate of homicide by those with schizophrenia and the overall homicide rate: a systematic review and meta-analysis. Schizophr Res, 112, 123-9.

Large, M. M. \& Nielssen, O. 2011. Violence in first-episode psychosis: a systematic review and meta-analysis. Schizophr Res, 125, 209-20.

Lataster, T., Myin-Germeys, I., Derom, C., Thiery, E. \& Van Os, J. 2009. Evidence that self-reported psychotic experiences represent the transitory developmental expression of genetic liability to psychosis in the general population. Am J Med Genet B Neuropsychiatr Genet, 150B, 1078-84.

Linscott, R. J. \& Van Os, J. 2013. An updated and conservative systematic review and meta-analysis of epidemiological evidence on psychotic experiences in children and adults: on the pathway from proneness to persistence to dimensional expression across mental disorders. Psychol Med, 43, 1133-49.

Lysaker, P. H., Meyer, P. S., Evans, J. D., Clements, C. A. \& Marks, K. A. 2001. Childhood sexual trauma and psychosocial functioning in adults with schizophrenia. Psychiatr Serv, 52, 1485-8.

Martin, G., Thomas, H., Andrews, T., Hasking, P. \& Scott, J. G. 2015. Psychotic experiences and psychological distress predict contemporaneous and future non-suicidal self-injury and suicide attempts in a sample of Australian school-based adolescents. Psychol Med, 45, 429-37.

Mojtabai, R. 2006. Psychotic-like experiences and interpersonal violence in the general population. Soc Psychiatry Psychiatr Epidemiol, 41, 183-90.

Morgan, V. A., Morgan, F., Galletly, C., Valuri, G., Shah, S. \& Jablensky, A. 2016. Sociodemographic, clinical and childhood correlates of adult violent victimisation in a large, national survey sample of people with psychotic disorders. Soc Psychiatry Psychiatr Epidemiol, 51, 269-79.

Mueser, K. T., Rosenberg, S. D., Goodman, L. A. \& Trumbetta, S. L. 2002. Trauma, PTSD, and the course of severe mental illness: an interactive model. Schizophr Res, 53, 123-43.

Nanni, V., Uher, R. \& Danese, A. 2012. Childhood maltreatment predicts unfavorable course of illness and treatment outcome in depression: a meta-analysis. Am J Psychiatry, 169, 141-51.

National Institute of Mental Health. 2017. Schizophrenia [Online]. Available: https://www.nimh.nih.gov/ health/topics/schizophrenia/index.shtml [Accessed 12-1-2017 2017].

Nederlof, A. F., Muris, P. \& Hovens, J. E. 2012. Psychotic-like experiences and aggressive behavior in a nonclinical sample. Personality and Individual Differences, 53, 33-37.

Newman, J. M., Turnbull, A., Berman, B. A., Rodrigues, S. \& Serper, M. R. 2010. Impact of traumatic and violent victimization experiences in individuals with schizophrenia and schizoaffective disorder. $J$ Nerv Ment Dis, 198, 708-14.

Nielssen, O. \& Large, M. 2010. Rates of homicide during the first episode of psychosis and after treatment: a systematic review and meta-analysis. Schizophr Bull, 36, 702-12.

Nishida, A., Sasaki, T., Nishimura, Y., Tanii, H., Hara, N., Inoue, K., Yamada, T., Takami, T., Shimodera, S., Itokawa, M., Asukai, N. \& Okazaki, Y. 2010. Psychotic-like experiences are associated with suicidal feelings and deliberate self-harm behaviors in adolescents aged 12-15 years. Acta Psychiatr Scand, 121, 301-7.

Nock, M. K., Joiner, T. E., Jr., Gordon, K. H., Lloyd-Richardson, E. \& Prinstein, M. J. 2006. Non-suicidal self-injury among adolescents: diagnostic correlates and relation to suicide attempts. Psychiatry Res, 144, 65-72. 
Nock, M. K. 2010. Self-injury. Annu Rev Clin Psychol, 6, 339-63.

Owen, P. R. 2012. Portrayals of schizophrenia by entertainment media: a content analysis of contemporary movies. Psychiatr Serv, 63, 655-9.

Palm, A., Danielsson, I., Skalkidou, A., Olofsson, N. \& Hogberg, U. 2016. Violence victimisation-a watershed for young women's mental and physical health. Eur J Public Health, 26, 861-867.

Perala, J., Suvisaari, J., Saarni, S. I., Kuoppasalmi, K., Isometsa, E., Pirkola, S., Partonen, T., Tuulio-Henriksson, A., Hintikka, J., Kieseppa, T., Harkanen, T., Koskinen, S. \& Lonnqvist, J. 2007. Lifetime prevalence of psychotic and bipolar I disorders in a general population. Arch Gen Psychiatry, 64, 19-28.

Perlis, R. H., Uher, R., Ostacher, M., Goldberg, J. F., Trivedi, M. H., Rush, A. J. \& Fava, M. 2011. Association between bipolar spectrum features and treatment outcomes in outpatients with major depressive disorder. Arch Gen Psychiatry, 68, 351-60.

Reagu, S., Jones, R., Kumari, V. \& Taylor, P. J. 2013. Angry affect and violence in the context of a psychotic illness: a systematic review and meta-analysis of the literature. Schizophr Res, 146, 46-52.

Saha, S., Scott, J. G., Johnston, A. K., Slade, T. N., Varghese, D., Carter, G. L. \& Mcgrath, J. J. 2011. The association between delusional-like experiences and suicidal thoughts and behaviour. Schizophr Res, 132, 197202.

Sharifi, V., Eaton, W. W., Wu, L. T., Roth, K. B., Burchett, B. M. \& Mojtabai, R. 2015. Psychotic experiences and risk of death in the general population: 24-27 year follow-up of the Epidemiologic Catchment Area study. Br J Psychiatry, 207, 30-6.

Strauss, J. S. 1969. Hallucinations and delusions as points on continua function. Rating scale evidence. Arch Gen Psychiatry, 21, 581-6.

Sullivan, S. A., Lewis, G., Gunnell, D., Cannon, M., Mars, B. \& Zammit, S. 2015. The longitudinal association between psychotic experiences, depression and suicidal behaviour in a population sample of adolescents. Soc Psychiatry Psychiatr Epidemiol, 50, 1809-17.

Sveticic, J. \& De Leo, D. 2012. The hypothesis of a continuum in suicidality: a discussion on its validity and practical implications. Ment IIIn, 4, e15.

Swanson, J. M. 1994. Mental disorder, substance abuse and community violence: an epidemiological approach. Violence and mental disorder. Chicago: University of Chicago Press.

Swanson, J. W., Borum, R., Swartz, M. S. \& Monahan, J. 1996. Psychotic symptoms and disorders and the risk of violent behaviour in the community. Criminal Behaviour and Mental Health, 6, 309-329.

Temmingh, H., Stein, D. J., Seedat, S. \& Williams, D. R. 2011. The prevalence and correlates of hallucinations in a general population sample: findings from the South African Stress and Health Study. Afr J Psychiatry (Johannesbg), 14, 211-7.

Ten Have, M., De Graaf, R., Van Weeghel, J. \& Van Dorsselaer, S. 2014. The association between common mental disorders and violence: to what extent is it influenced by prior victimization, negative life events and low levels of social support? Psychol Med, 44, 1485-98.

Van Nierop, M., Van Os, J., Gunther, N., Myin-Germeys, I., De Graaf, R., Ten Have, M., Van Dorsselaer, S., Bak, M. \& Van Winkel, R. 2012. Phenotypically continuous with clinical psychosis, discontinuous in need for care: evidence for an extended psychosis phenotype. Schizophr Bull, 38, 231-8.

Van Os, J., Hanssen, M., Bijl, R. \& Ravelli, A. 2000. Straus (1969) revisited: A psychosis continuum in the general population? Schizophrenia Research, 45, 11-20.

Van Os, J., Linscott, R. J., Myin-Germeys, I., Delespaul, P. \& Krabbendam, L. 2009. A systematic review and meta-analysis of the psychosis continuum: evidence for a psychosis proneness-persistence-impairment model of psychotic disorder. Psychol Med, 39, 179-95.

Van Os, J. \& Linscott, R. J. 2012. Introduction: The extended psychosis phenotype--relationship with schizophrenia and with ultrahigh risk status for psychosis. Schizophr Bull, 38, 227-30.

Van Os, J. \& Reininghaus, U. 2016. Psychosis as a transdiagnostic and extended phenotype in the general population. World Psychiatry, 15, 118-24.

Varese, F., Smeets, F., Drukker, M., Lieverse, R., Lataster, T., Viechtbauer, W., Read, J., Van Os, J. \& Bentall, R. P. 2012. Childhood adversities increase the risk of psychosis: a meta-analysis of patient-control, prospective- and cross-sectional cohort studies. Schizophr Bull, 38, 661-71. 
Werbeloff, N., Dohrenwend, B. P., Yoffe, R., Van Os, J., Davidson, M. \& Weiser, M. 2015. The association between negative symptoms, psychotic experiences and later schizophrenia: a population-based longitudinal study. PLoS One, 10, e0119852.

Wigman, J. T., Van Nierop, M., Vollebergh, W. A., Lieb, R., Beesdo-Baum, K., Wittchen, H. U. \& Van Os, J. 2012. Evidence that psychotic symptoms are prevalent in disorders of anxiety and depression, impacting on illness onset, risk, and severity--implications for diagnosis and ultra-high risk research. Schizophr Bull, 38, 247-57.

Wigman, J. T., Van Os, J., Abidi, L., Huibers, M. J., Roelofs, J., Arntz, A., Kelleher, I. \& Peeters, F. P. 2014. Subclinical psychotic experiences and bipolar spectrum features in depression: association with outcome of psychotherapy. Psychol Med, 44, 325-36.

World Health Organization 2013. Global and regional estimates of violence against women: prevalence and health effects of intimate partner violence and non-partner sexual violence, Geneva, World Health Organization.

World Health Organization 2014. Preventing suicide: A global imperative, Geneva, World Health Organization.

Yu, R., Geddes, J. R. \& Fazel, S. 2012. Personality disorders, violence, and antisocial behavior: a systematic review and meta-regression analysis. J Pers Disord, 26, 775-92. 



\section{Chapter}

\section{Psychotic experiences and risk of self- injurious behaviour in the general population: a systematic review and meta-analysis}

Steven Honings ${ }^{1}$, Marjan Drukker ${ }^{1}$, Robin Groen ${ }^{2}$, Jim van Os ${ }^{1,3,4}$

1. Department of Psychiatry and Psychology, South Limburg Mental Health Research and Teaching Network, Maastricht University Medical Centre, Maastricht, the Netherlands.

2. Student, Faculty of Psychology and Neuroscience, Maastricht University, the Netherlands

3. King's College London, King's Health Partners, Department of Psychosis Studies, Institute of Psychiatry, London, UK

4. Department of Psychiatry, Brain Center Rudolf Magnus Institute, University Medical Center Utrecht, Utrecht, The Netherlands.

Published as:

Honings, S., Drukker, M., Groen, R. \& Van Os, J. 2016. Psychotic experiences and risk of self-injurious behaviour in the general population: a systematic review and metaanalysis. Psychol Med, 46, 237-51. 


\begin{abstract}
Background: Recent studies suggest that psychotic experiences (PE) in the general population are associated with an increased risk of self-injurious behaviour. Both the magnitude of this association and the level of adjustment for confounders vary among studies. A meta-analysis was performed to integrate the available evidence. The influence of possible confounders, including variably defined depression, was assessed.
\end{abstract}

Method: A systematic review and meta-analysis was conducted including general population studies reporting on the risk of self-injurious behaviour in individuals with PE. Studies were identified by a systematic search strategy in Pubmed, PsycINFO and Embase. Reported effect sizes were extracted and meta-analytically pooled.

Results: The risk of self-injurious behaviour was 3.20 times higher in individuals with PE compared to those without. Sub-analyses showed that PE were associated with selfharm, suicidal ideation as well as suicidal attempts. All studies had scope for considerable residual confounding; effect sizes adjusted for depression were significantly smaller than effect sizes unadjusted for depression. In the longitudinal studies, adjustment for psychopathology resulted in a 74\% reduction in excess risk.

Conclusion: PE are associated with self-injurious behaviour, suggesting they have potential as passive markers of suicidality. However, the association is confounded and several methodological issues remain, particularly how to separate PE from the full range of connected psychopathology in determining any specific association with self-injurious behaviour. Given evidence that PE represent an indicator of severity of non-psychotic psychopathology, the association between PE and self-injurious behaviour likely reflects greater likelihood of self-injurious behaviour in more severe states of mental distress. 


\section{Introduction}

Self-injurious behaviour comprises various phenomena, including non-suicidal selfinjury, suicidal ideation, suicide attempts and completed suicide (Nock 2010). Recently, several studies found that psychotic experiences (PE) are associated with an increased risk of self-injurious behaviour (DeVylder et al. 2015a; Jang et al. 2014; Kelleher et al. 2013b; Martin et al. 2015; Nishida et al. 2014; Saha et al. 2011).

PE represent delusional or hallucinatory experiences that fall below the threshold of a diagnosable psychotic disorder, in absence of significant psychological distress or need for help (Linscott and van Os 2013; Kelleher et al. 2012a). Reliable PE estimates can best be obtained with clinical interviews containing sufficient PE items. Results from selfreports (including lay interviewers writing down self-reports) and interviews with fewer than 10 items are less reliable (Linscott and van Os 2013). Significant variation across ethnicities in both PE endorsement and in self-reported attributions and understandings for these experiences has been reported, which may result from culturally sanctioned beliefs and idioms of distress (Earl et al. 2015). Evidence suggests that PE are part of an extended psychosis phenotype ranging from psychotic experiences in the general population to full-blown psychosis, sharing the same demographic, etiological, familial and psychopathological correlates (van Os et al. 2009). The concept of a psychosis continuum is supported by fact that the PE population prevalence (7.2\%) (Linscott and van Os 2013 ) is much higher than the population prevalence of broadly defined psychotic disorders (3.48\%) (Perala et al. 2007).

Population-based research indicates that the majority of individuals with PE either have a diagnosable DSM-IV-mental disorder or significant mental health difficulties in the absence of a diagnosis (Jeppesen et al. 2015; van Os et al. 2000; DeVylder et al. 2014; Kelleher et al. 2012a). Prospective research indicates that PE are a risk factor for several mental health outcomes including psychotic disorder (Linscott and van Os 2013) and other mental disorders (Kelleher et al. 2012a; Kaymaz et al. 2012), while the presence of PE in non-psychotic mental disorders has been identified as an indicator of illness severity and poor outcome (Wigman et al. 2012; Wigman et al. 2014; Perlis et al. 2011; Guloksuz et al. 2015; Kelleher et al. 2012a).

With more than 800.000 completed suicides and more than sixteen million suicide attempts each year worldwide, suicidality constitutes a major global health burden (World Health Organization 2014). Suicidality has been conceptualized as a continuum developing from suicidal ideation (i.e. having thoughts about killing oneself) to suicide plans (i.e. considering a specific method through which one intends to die), to suicide attempts (i.e. engagement in potentially self-injurious behaviour in which there is some intent to die), and completed suicide (Sveticic and De Leo 2012; Nock 2010). The construct of a continuum of suicidality is supported by epidemiological data showing a decreasing twelve month prevalence when moving from one end of the spectrum to the other, ranging from $3.3 \%$ for suicidal ideation to $0.01 \%$ for completed suicide (Kessler et al. 2005). 
Forms of self-injurious behaviour without suicidal intent also exist. An obstacle in the literature is the inconsistent use of definitions and terminology when referring to self-injurious behaviour without suicidal intent, including self-mutilation, deliberate selfharm and parasuicide (Nock 2010). In the fifth edition of the Diagnostic and Statistical Manual of Mental Disorders (DSM-5), non-suicidal self-injury (NSSI) has been proposed as a new diagnosis for self-injurious behaviour without suicidal intent (American Psychiatric Association 2013). Since suicidal intent as a dimensional phenomenon may be difficult to assess, researchers often classify self-injurious behaviour as suicidal when suicidal intent cannot be excluded to prevent underestimation of the severity (Nock 2010). Although non-suicidal self-harm is an important risk factor for future suicidal behaviour (Hawton et al. 2012; Hawton and van Heeringen 2009), it remains unclear whether non-suicidal self-harm and suicide are phenomena on the same continuum, as some authors suggest (Hamza et al. 2012).

PE have been associated with self-injurious behaviour, but most studies were crosssectional (Capra et al. 2015; DeVylder et al. 2015a; Jang et al. 2014; Kelleher et al. 2012b; Nishida et al. 2010; Saha et al. 2011; Temmingh et al. 2011). Five longitudinal studies with variable length of follow-up (Fisher et al. 2013; Kelleher et al. 2013b; Sharifi et al. 2015; Martin et al. 2015; Sullivan et al. 2015) showed contradictory results, ranging from absence of an association (Martin et al. 2015; Sharifi et al. 2015) to an odds ratio of 11.27 (95\% Cl 4.44 - 28.62) (Kelleher et al. 2013b). Martin et al. (2015) conducted the only longitudinal study to date assessing PE in relation to NSSI and found no association in absence of psychological distress.

All phenomena of self-injurious behaviour are strongly associated with mental disorders. Of all adolescents engaging in NSSI, 87.6\% met criteria for a DSM-IV diagnosis and $41.6 \%$ for a major depressive disorder (Nock et al. 2006). Furthermore, a mental disorder was present in $91 \%$ of the individuals that committed suicide; most common diagnoses were depressive disorders, substance abuse and schizophrenia (Cavanagh et al. 2003). This means that both depression and depressive symptoms are associated with self-injurious behaviour. PE are also prevalent across a range of mental disorders, including depression. Therefore, any association between PE and self-injurious behaviour is likely confounded by the presence of depressive symptoms and other mental disorders. Various studies reported that the specific association between PE and selfinjurious behaviour remained even after including depression in the regression model (Jang et al. 2014; Calkins et al. 2014; DeVylder et al. 2015a; Lewis-Fernandez et al. 2009; Nishida et al. 2014; Olfson et al. 2002; Polanczyk et al. 2010; Saha et al. 2011). However, controlling for a variable (i.e. depression) that is strongly associated with the outcome (i.e. self-injurious behaviour), although often carried out, is methodologically challenging in cross-sectional designs (Miller and Chapman 2001). For example, if suicidality forms a fundamental part of the depression syndrome, it is conceptually impossible, and therefore methodologically questionable, to examine how suicidality varies in relation to PE whilst keeping depression constant. Longitudinal studies have more possibili- 
ties to examine the association between PE and self-injurious behaviour, whilst controlling for depression, although similar methodological challenges exist.

Other methodological issues may require attention. First, depression is best represented as a dimensional phenotype (Anderson et al. 1993; Kendler and Gardner 1998), and correcting for a narrow dichotomous measure of depression at the level of the clinical phenotype, although often done, leaves more room for residual confounding than correcting for a broad dimensional measure of depression. Second, while correction for depression may be required, many more features of psychopathology may either confound or mediate the association between PE and suicidality. Furthermore, given that PE are an indicator of underlying severity of non-psychotic psychopathology (Wigman et al. 2012; Wigman et al. 2014; Perlis et al. 2011; Guloksuz et al. 2015; Kelleher et al. 2012a), the association between PE and suicidality may simply reflect greater likelihood of suicidality as a function of greater psychopathology severity.

Despite the wide variety of available studies in unselected general population samples, no systematic review or meta-analysis has been performed. A systematic review and meta-analysis of all previous studies is required, given the fact that reported effect sizes in both cross-sectional and longitudinal studies show marked variation and issues with confounding remain. The aim of the present meta-analysis is to integrate the available evidence on the association between PE in the general population and selfinjurious behaviour from both cross-sectional and longitudinal studies. Moreover, it will assess to what degree this association can be considered confounded by depression and other measures of psychopathology.

\section{Methods}

\section{Definitions}

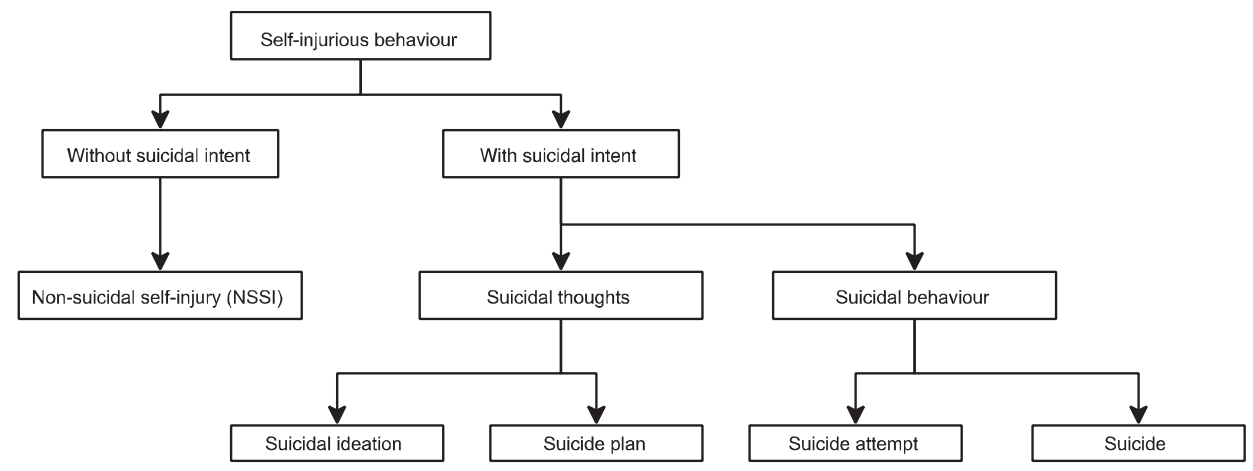

Figure 1: Classification of self-injurious behaviour.

Self-injurious behaviour was categorised in terms of presence or absence of suicidal intent, in agreement with previous work (Fig. 1) (Nock 2010). Self-injurious behaviour 
with suicidal intent was further divided into suicidal thoughts and suicidal behaviour. Suicidal thoughts included all cognitions about suicide without engagement in selfharming behaviour, including suicidal ideation, suicidal feelings and suicide plans. Suicidal behaviour included all outcomes in which an individual physically engaged in selfinjurious behaviour with suicidal intent, including suicidal attempts and completed suicide. Self-injurious behaviour was only classified as non-suicidal self-injury if the absence of suicidal intent was specified.

\section{Search strategy and selection criteria}

The Meta-analysis of Observational Studies in Epidemiology (MOOSE) guidelines were followed. A literature search without restriction by publication date or any other filters was conducted in Pubmed, PsycINFO and Embase using the following terms: (Psychotic exp* OR subclinical psych* OR subthreshold psych* OR attenuated psych* OR psychotic-like OR psychotic sympt* OR delusion* OR hallucinat* OR schizotyp*) AND (suic* OR parasuic* OR self-harm OR selfharm OR self-injur* OR self injur*). This resulted in 4362 studies after removal of duplicates. The literature searches were handled using bibliographic citation management software (EndNote $\mathrm{X} 7$ ). Papers were first screened by reading the title and subsequently by reading abstracts and full texts. All steps of this process were done by two independent reviewers $(\mathrm{SH}, \mathrm{RG})$. In case of conference abstracts without available full articles, authors were contacted to retrieve preliminary data, unpublished or submitted articles. The literature search was complemented by hand searching reference lists of obtained articles.

Papers were included in the meta-analysis if they: a) reported the association between PE and self-injurious behaviour; b) were conducted in a general population sample; c) were written in the Dutch, English or German language. Studies were excluded if they: a) were conducted in clinical or mental health populations (including Ultra High Risk populations); b) did not contain quantitative data (i.e. qualitative studies).

\section{Data-extraction}

The data-extraction was performed by two independent reviewers ( $\mathrm{SH}, \mathrm{RG}$ ) using a standardized data extraction form. Relevant exposure variables, outcome variables, associated effect sizes and their 95\% confidence intervals ( $\mathrm{Cl}$ ) or the standard errors of the effect sizes were recorded. If a study reported multiple effect sizes for the same exposure and outcome combination but with varying degrees of adjustment, all effect sizes were recorded, together with information on the level of adjustment. If a study reported on multiple relevant outcomes, all outcomes and effect sizes were recorded. When the association between PE and self-injurious behaviour was not reported but could be derived from the available data, both reviewers calculated the odds ratios. In 
case of disagreement after data-extraction, consensus was reached between the two reviewers and when necessary a third, senior, reviewer was consulted (MD).

\section{Statistical analysis}

All analyses were performed using Stata 13 using the METAN command to calculate metaanalytic odds ratios and $95 \% \mathrm{Cl}$. Since most studies reported odds ratios as effect sizes, these were used in the analyses. If instead of odds ratios, hazard ratios or relative risks were reported, we used these as substitutes for the odds ratio since the prevalence of the outcome of interest is low. A main analysis was performed including all outcomes with regard to self-injurious behaviour. For this analysis, one effect size per study was selected. Thus, if studies reported effect sizes for multiple outcomes, the association with the most severe outcome variable was selected for the main analysis in the following order of severity: a) completed suicide; b) attempted suicide; c) suicide plans; d) suicidal ideation; e) non-suicidal self-harm. When a study reported multiple effect sizes for identical exposures and outcomes, the most completely adjusted effect size was selected for the main analysis. Studies reporting odds ratios for continuous exposure and/or outcome measures were excluded from the main analysis and analysed separately.

Meta-analytic sub-analyses were conducted for NSSI, suicidal thoughts (i.e. suicidal ideation or suicide plans) and suicidal behaviour (i.e. suicide attempts or suicide). Moreover, a separate sub-analysis for studies reporting effect sizes for continuous exposure and/or outcome measures was carried out. For this purpose, the reported regression coefficient and standard errors were used. If studies reported odds ratios instead of regression coefficients, the odds ratios and their corresponding $95 \% \mathrm{Cl}$ were converted to regression coefficients. The regression coefficients were standardized using the standard deviation (SD) of the exposure and outcome with the following formula: $\beta=B *\left(S D_{\text {exposure }} / S D_{\text {outcome }}\right)$, in which $B$ is the unstandardized regression coefficient and $\beta$ is the standardized regression coefficient. If the association represented a continuous exposure variable and a dichotomous outcome variable, $\mathrm{SD}_{\text {outcome }}$ was replaced by the standard deviation of the standard logistic distribution ( $\pi / \sqrt{ } 3$ ) (Katz 2006; Menard 2004; Weiner et al. 2003).

To check the potential for bias arising from the method of selecting one result per study rather than any of the other reported results in that same study, a sensitivity analysis was conducted using permutations to test robustness of the analyses. One effect size per study was randomly selected from all available rows in the database and a metaanalysis was performed. This process was repeated a thousand times, thus generating thousand meta-analytical results per analysis. Finally, these thousand regression coefficients were averaged and converted into one overall odds ratio and confidence interval.

To assess whether the association between PE and self-injurious behaviour remained after controlling for depression, meta-analyses were performed using only studies that controlled for depression. To further assess the influence of adjustment for depression 
and other confounders on the reported effect sizes, meta-regression analysis was performed using the METAREG routine in Stata. Since the association between PE and selfinjurious behaviour and the influence of adjustment for confounding can be estimated most accurately from longitudinal studies, separate analyses were done for the longitudinal studies before and after adjustment. Finally, in order to identify possible publication bias, funnel plots, Egger's tests and trim-and-fill procedures were applied.

\section{Results}

\section{Characteristics of included studies}

The systematic search strategy resulted in twenty-five studies (Fig. 2). Characteristics of these studies are shown in Table 1. Most of the included studies used a cross-sectional study design ( $n=20)$; some used a longitudinal design $(n=5)$. A great variety of questionnaires and tests was used to examine PE and self-injurious behaviour. The level of adjustment varied among studies. Most studies controlled for demographic characteristics. Fewer studies adjusted their analyses for the presence of one or more mental disorders including depressive disorder. Only two studies (Jang et al. 2014; Polanczyk et al. 2010) controlled for broader measures of depressive symptoms.

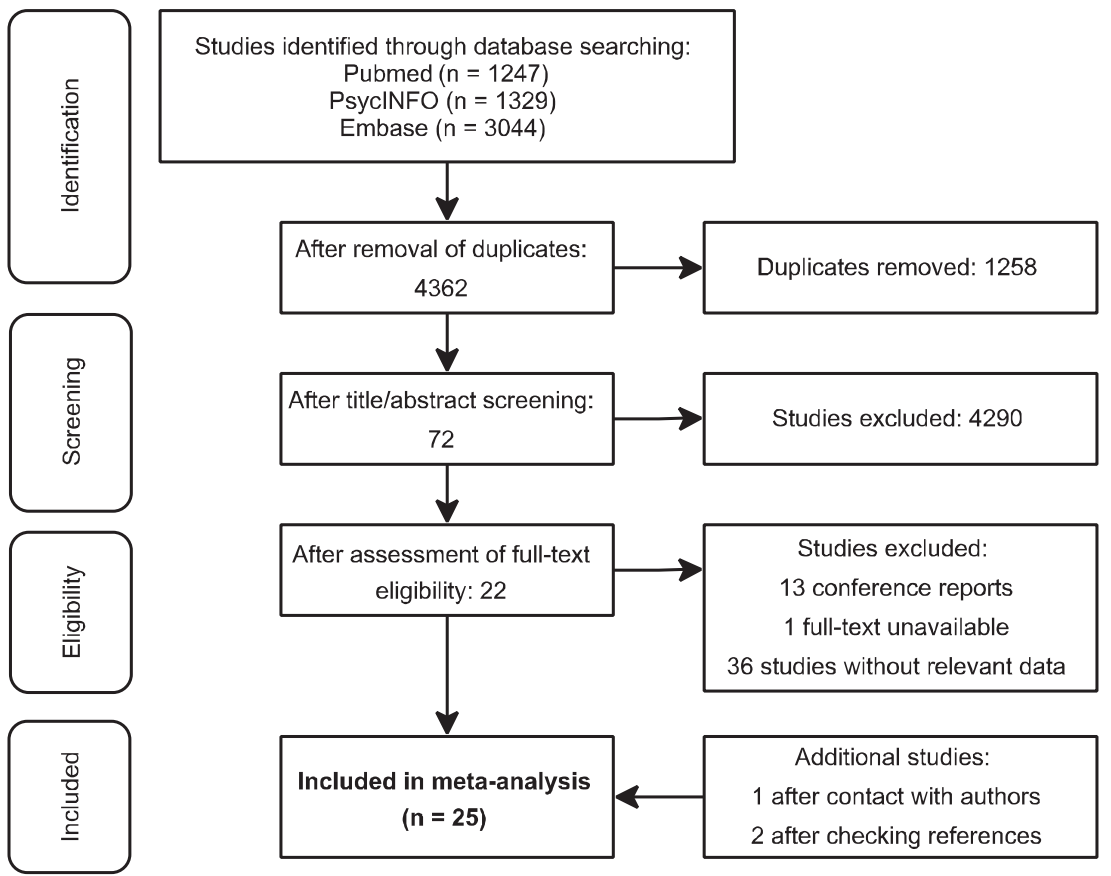

Figure 2: Flowchart of literature search 
Psychotic experiences and risk of self-injurious behaviour

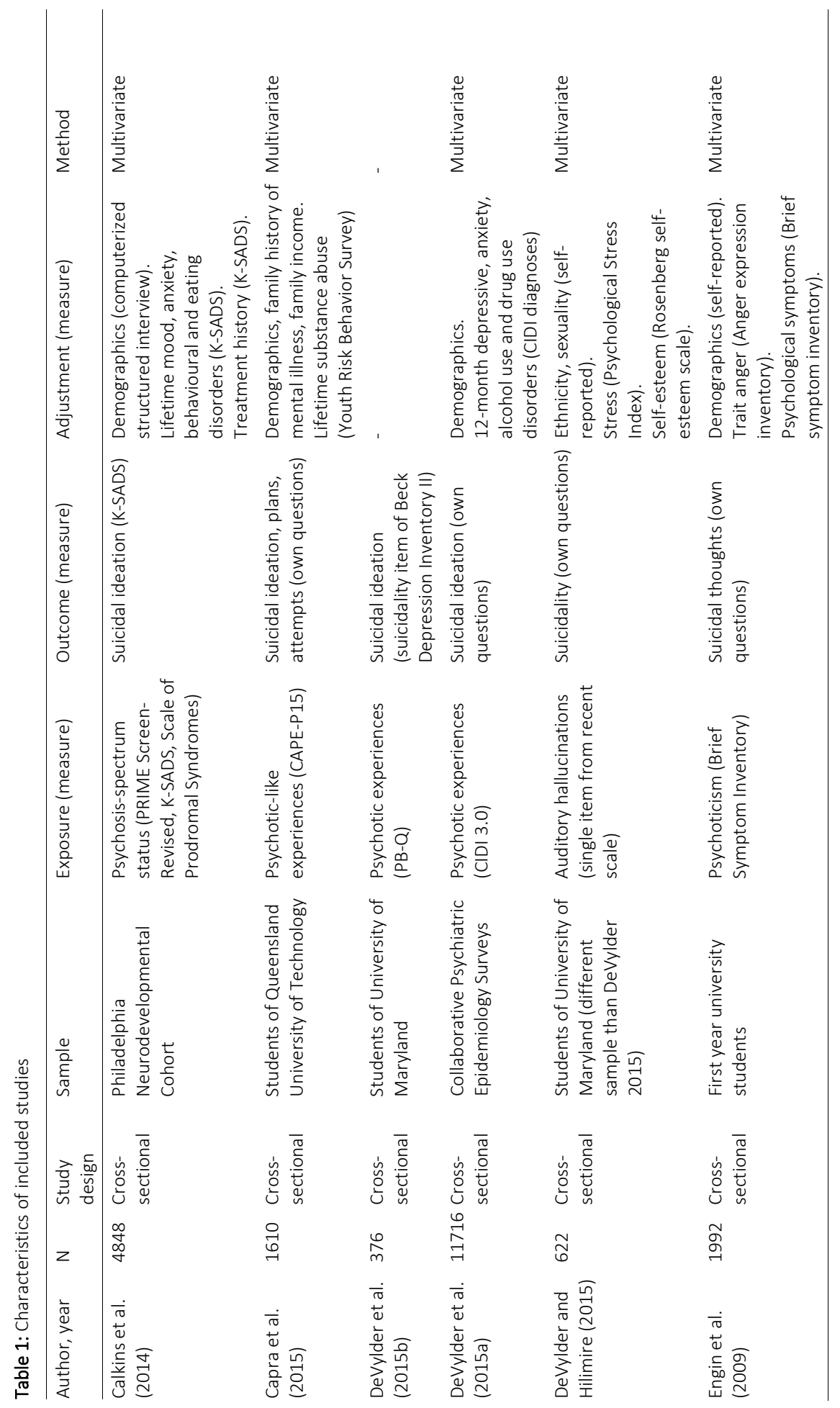




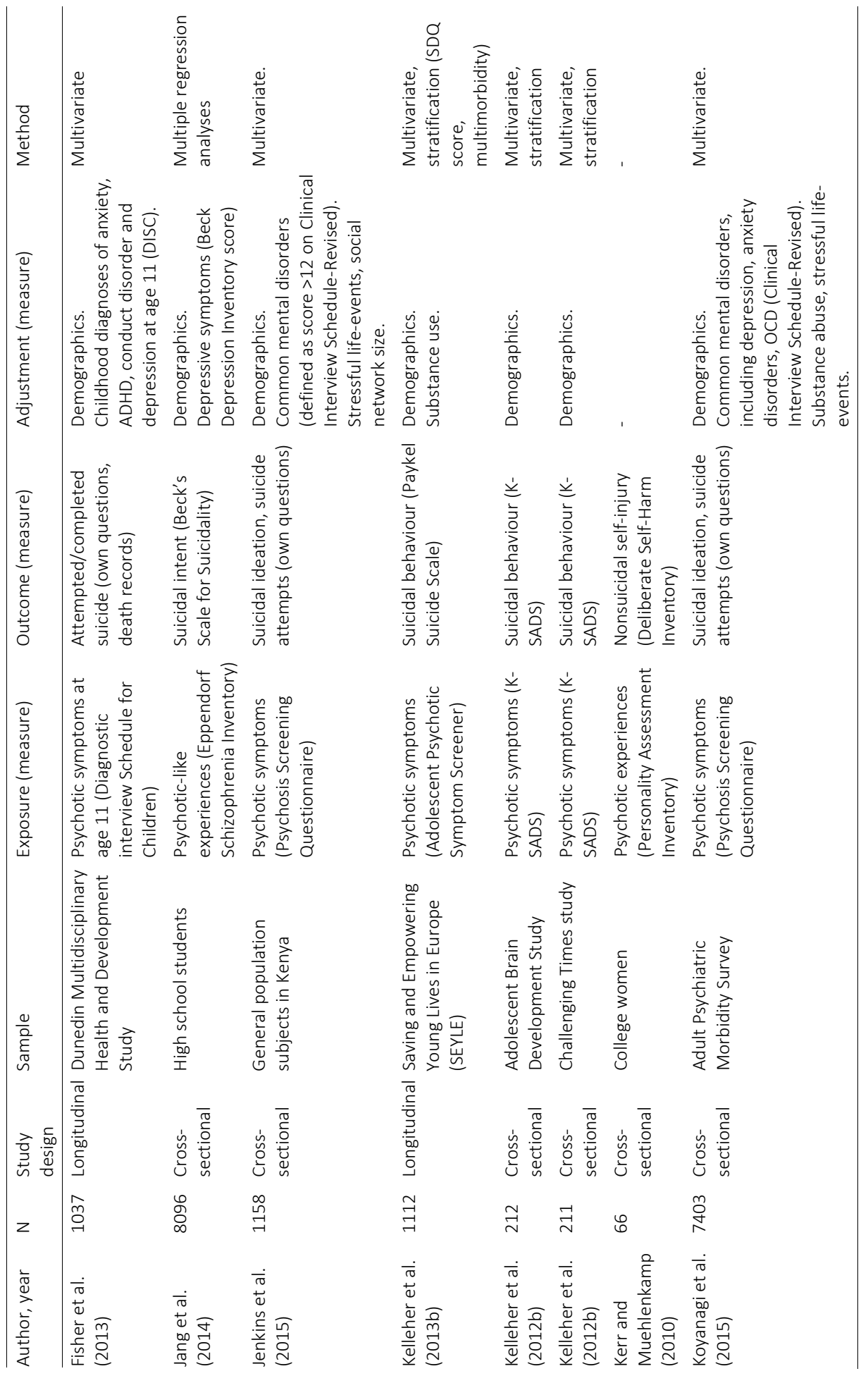


Psychotic experiences and risk of self-injurious behaviour

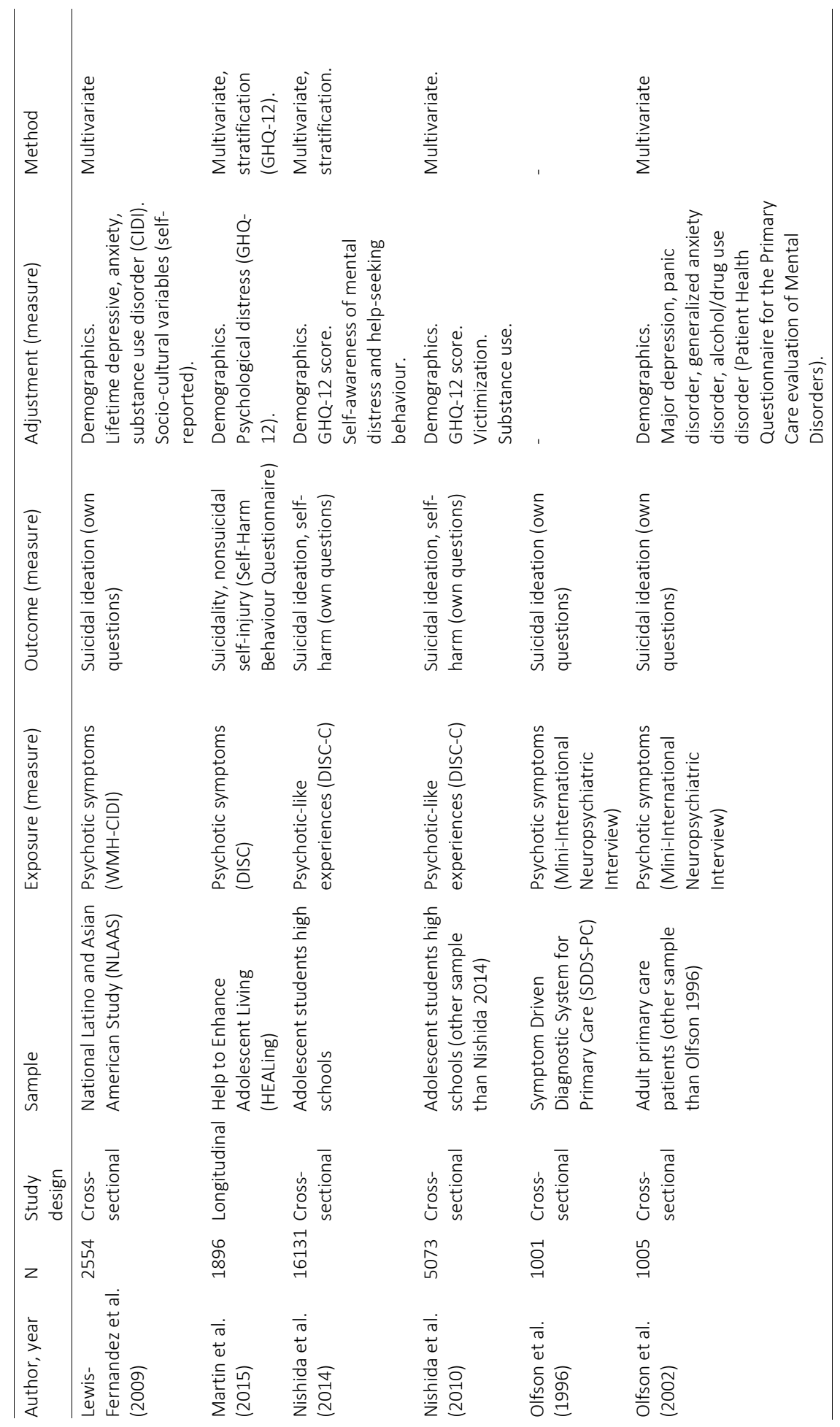


Chapter 2

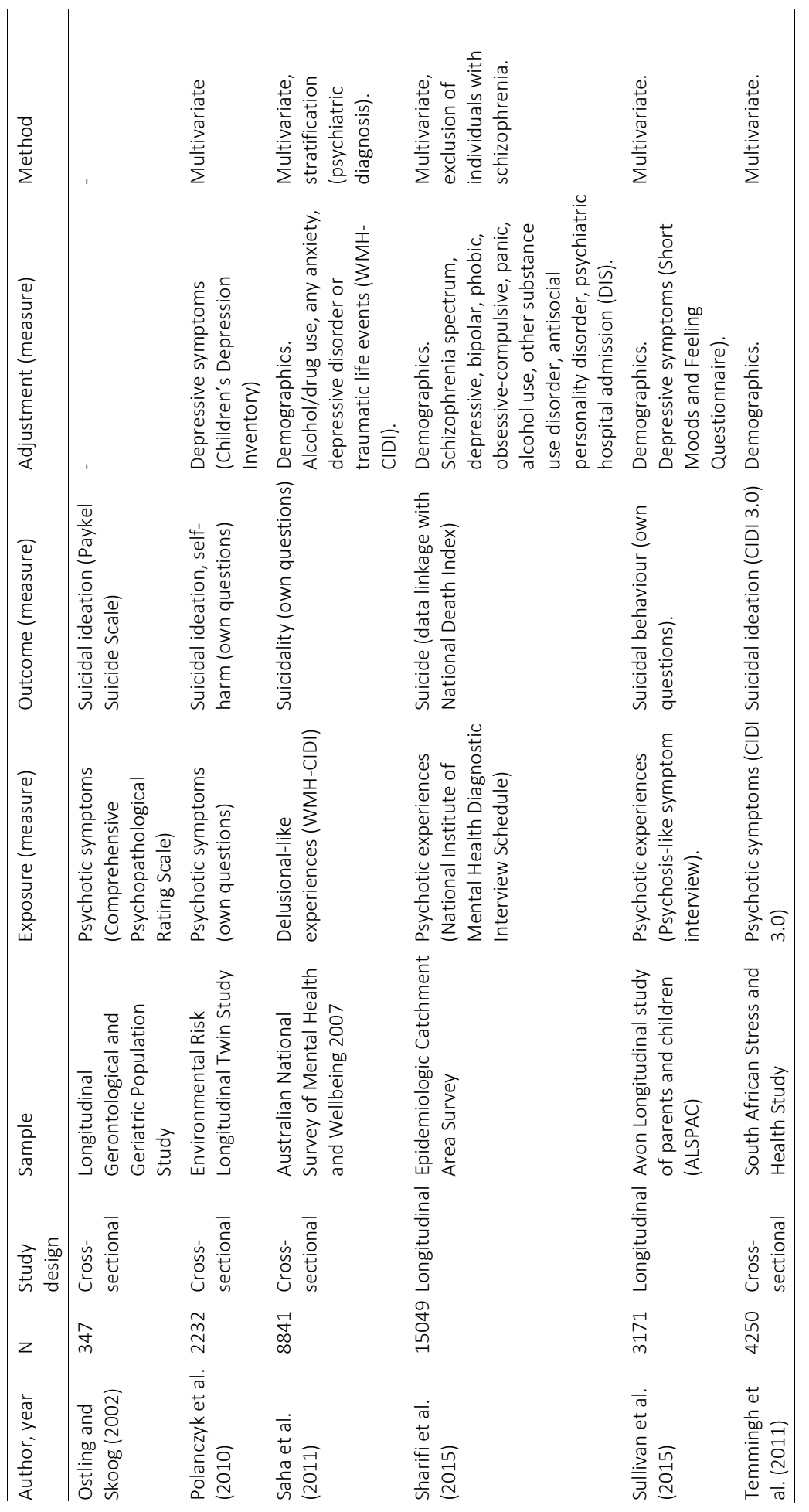




\section{Self-injurious behaviour}

Twenty of the included studies reported effect sizes for the association between PE and a form of self-injurious behaviour (Fig. 3). The pooled odds ratio of this association was $3.20(95 \% \mathrm{Cl} 2.33-4.40)$, indicating that experiencing PE is associated with significantly increased odds of self-injurious behaviour. The funnel plot (Fig. 4), Egger's test and the trim-and-fill procedure showed no evidence of publication bias.

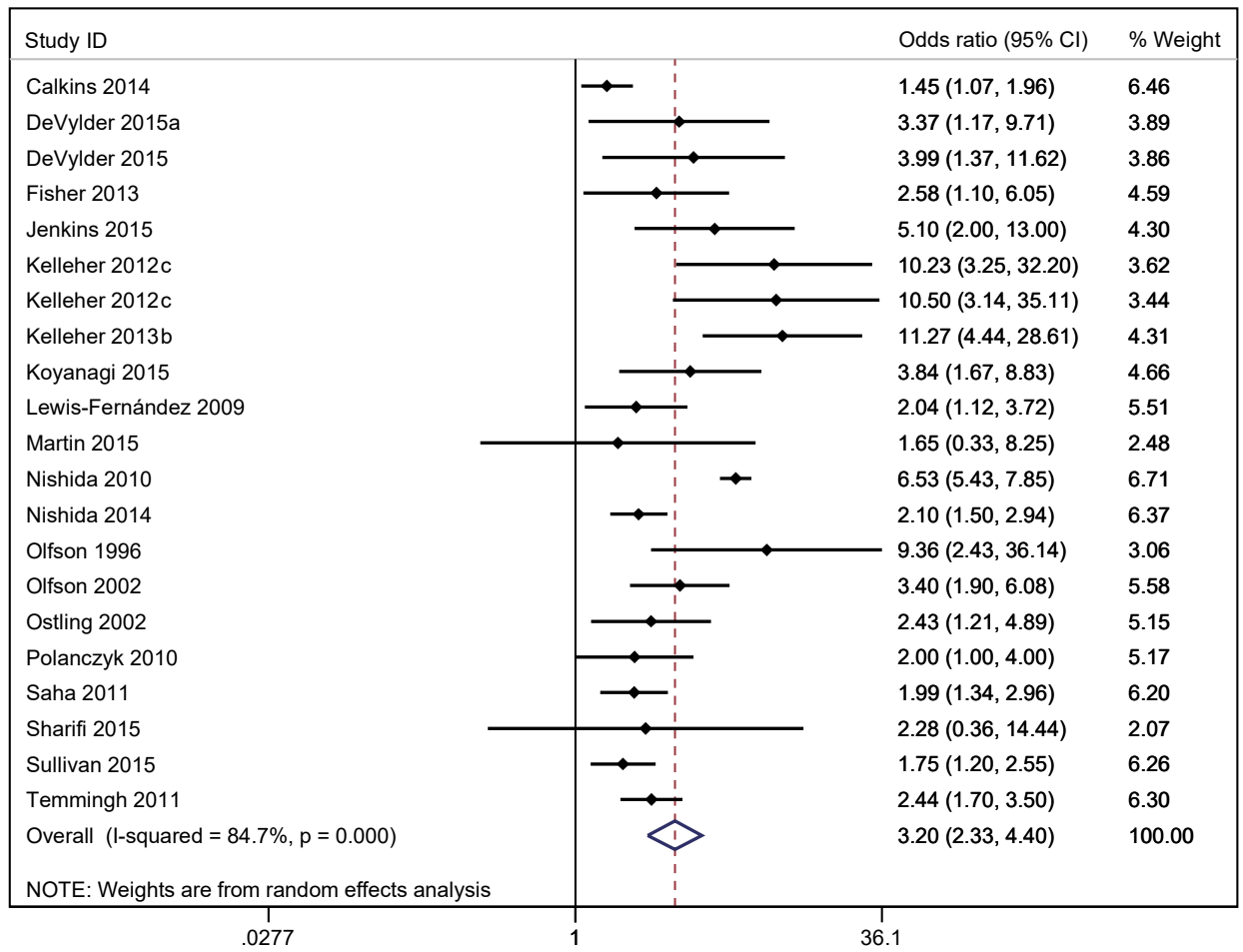

Figure 3: Meta-analysis of studies reporting on the association between psychotic experiences and selfinjurious behaviour.

\section{Non-suicidal self-injurious behaviour}

Two studies specifically examined the association between PE and NSSI (Martin et al. 2015; Kerr and Muehlenkamp 2010). Because one of these studies (Kerr and Muehlenkamp 2010) used a case-control design with a continuous measure for PE, meta-analysis could not be performed. Kerr and Muehlenkamp (2010) compared women with NSSI to women without NSSI and found that those with NSSI scored significantly higher on a scale measuring PE, although PE did not predict NSSI group membership in logistic regression analysis. Martin et al. (2015) assessed the association between PE and NSSI using both cross-sectional and longitudinal analyses. The cross-sectional exam- 
ination showed that experiencing PE increased the risk of NSSI, even after adjustment for age and gender ( $\mathrm{OR}=2.88,95 \% \mathrm{Cl} 1.51-5.49)$. When individuals reported psychological distress alongside PE, the association between PE and NSSI was stronger (OR=17.81, 95\% Cl 10.00 - 31.71). In a longitudinal design, experiencing PE without psychological distress was not associated with an increased risk of NSSI one year later. Exposure to both PE and psychological distress remained significantly associated with an increased NSSI risk in the longitudinal design (OR=11.45, 95\% Cl 5.70-23.00).

Funnel plot with pseudo $95 \%$ confidence limits

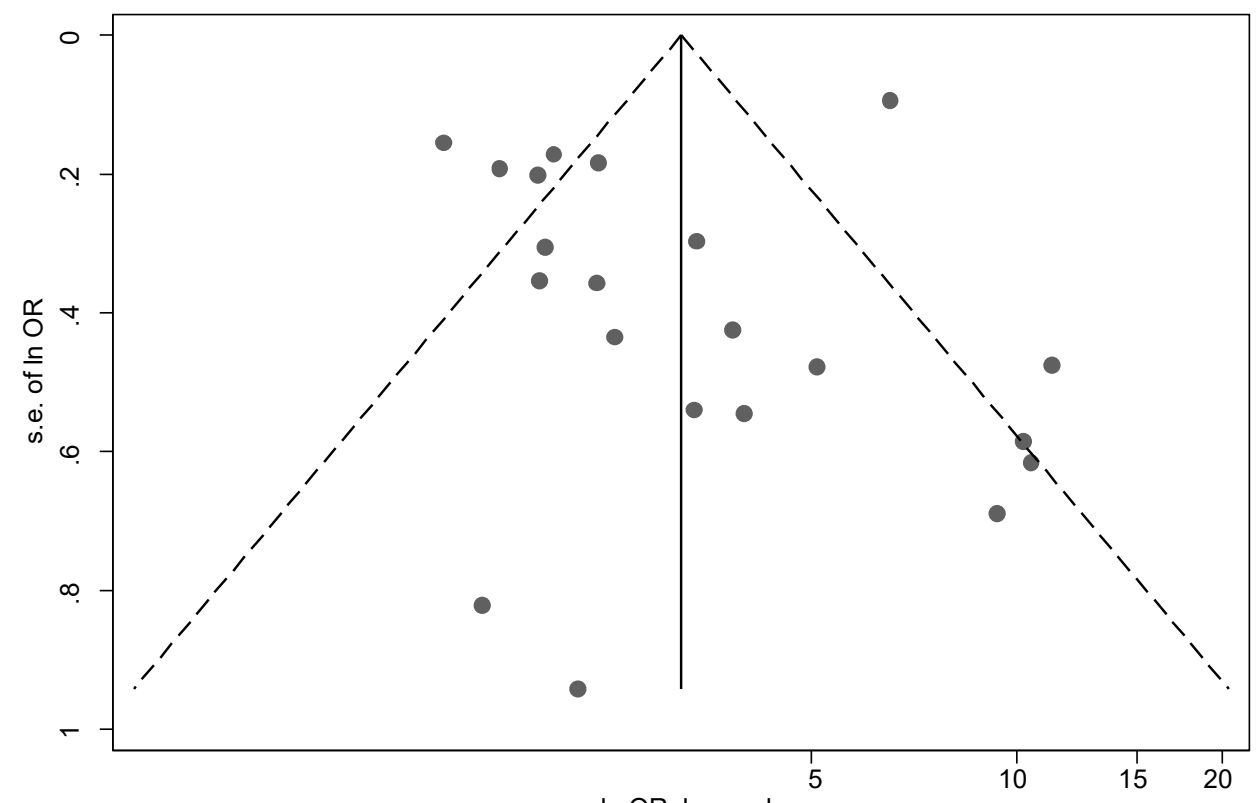

In OR, log scale

Figure 4: Funnel plot of meta-analysis on the association between psychotic experiences and self-injurious behaviour.

Two other cross-sectional studies reported on the association between PE and selfharm, but did not specify whether there was suicidal intent or not. A meta-analysis including all three studies (Martin et al. 2015; Nishida et al. 2010; Nishida et al. 2014) resulted in a pooled odds ratio of $2.36(95 \% \mathrm{Cl} 1.13-4.95)$ (Fig. 5), indicating that experiencing PE is associated with significantly increased odds of self-harm. Removing an outlier from the study (Nishida et al. 2010) lowered the pooled odds ratio to 1.61 (95\% $\mathrm{Cl} 0.95-2.73$ ) and rendered it statistically non-significant. Egger's test and the trimand-fill procedure showed no evidence of possible publication bias. 


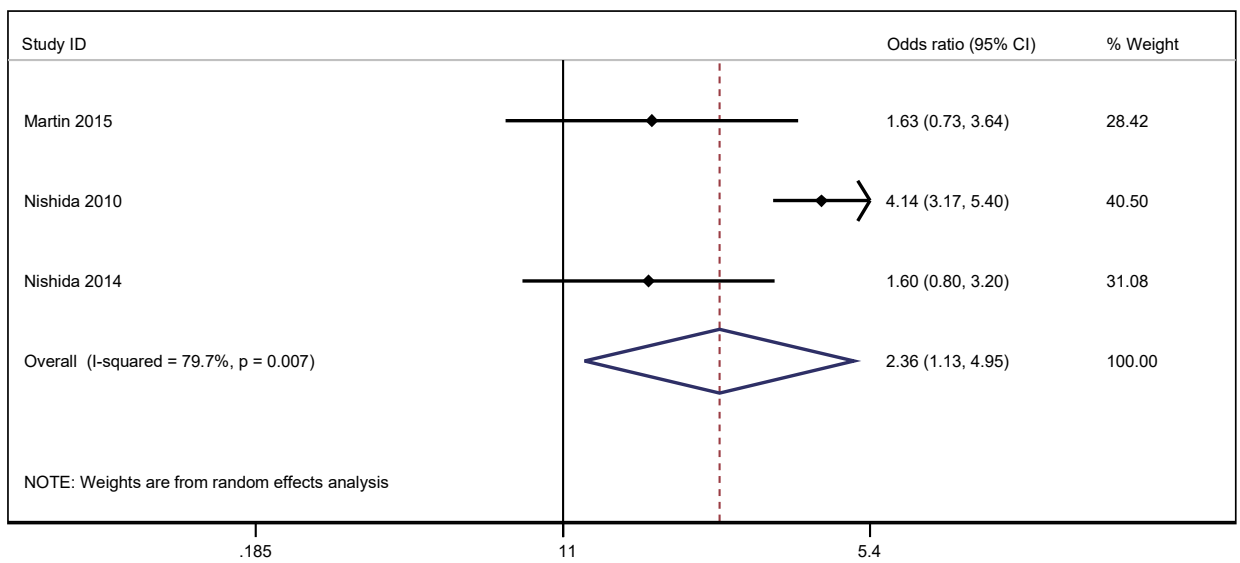

Figure 5: Meta-analysis of studies reporting on the association between psychotic experiences and self-harm.

\section{Suicidal self-injurious thoughts and behaviour}

When suicidal thoughts (i.e. suicidal ideation or suicidal plans) was analysed separately, the pooled odds ratio was 2.47 (95\% Cl $1.71-3.59)$ (Fig. 6). After removal of two outliers the pooled odds ratio decreased to $2.06(95 \% \mathrm{Cl} 1.69-2.52)$, but remained statistically significant. When the association between PE and suicidal behaviour (i.e. suicidal attempts or completed suicides) was analysed, the pooled odds ratio was $3.03(95 \% \mathrm{Cl}$ 2.08 - 4.41) (Fig. 7). Although Egger's test was statistically non-significant for both analyses, the trim-and-fill procedure decreased both pooled odds ratios, indicating possible publication bias. However, results remained statistically significant.

Three studies reported on the association between PE and suicidal behaviour using a continuous exposure measure (score on scale measuring psychotic experiences) and a dichotomous outcome measure (presence/absence of suicidal self-injurious behaviour). The pooled odds ratio was $1.34(1.24-1.45)$ (Fig. 8), indicating that an increase of one standard deviation on a scale measuring psychotic experiences is associated with $34 \%$ increase in the odds of suicidal self-injurious behaviour. 


\section{Chapter 2}

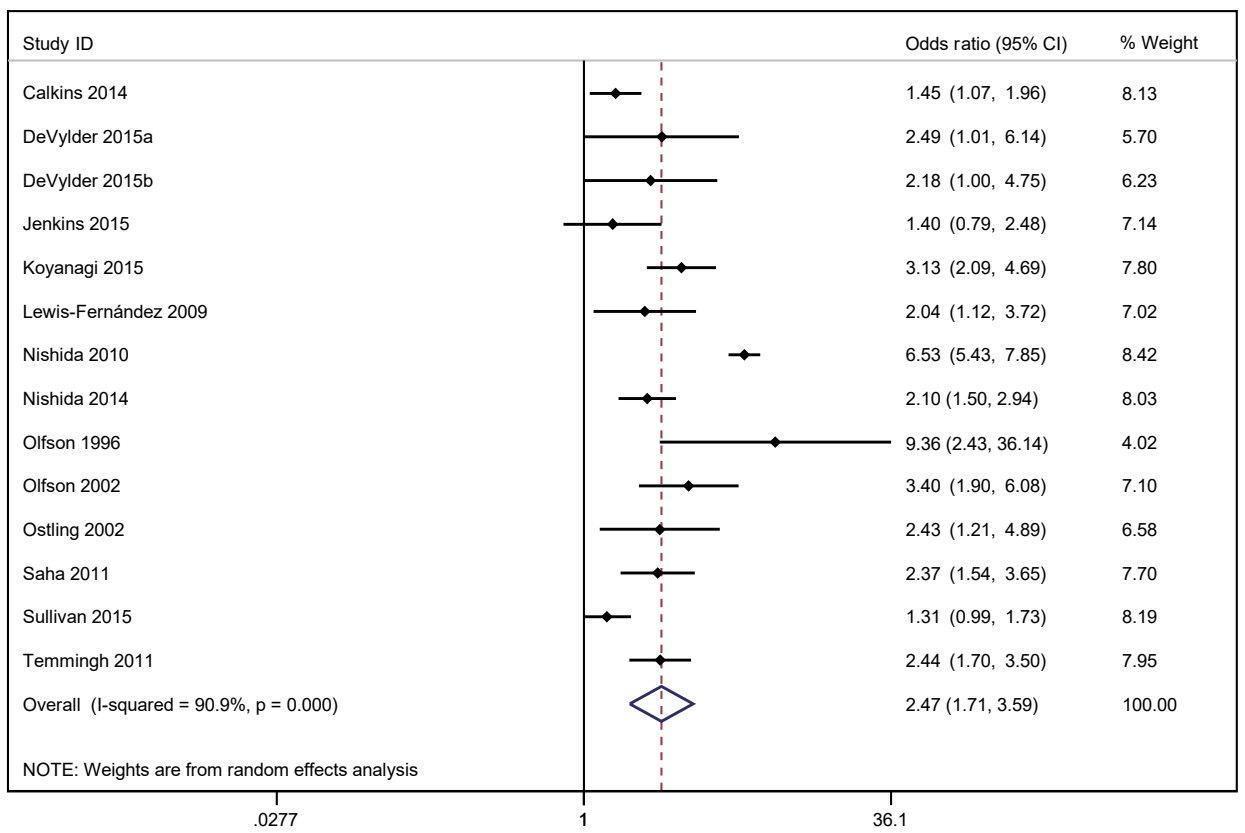

Figure 6: Meta-analysis of the association between psychotic experiences and suicidal ideation.

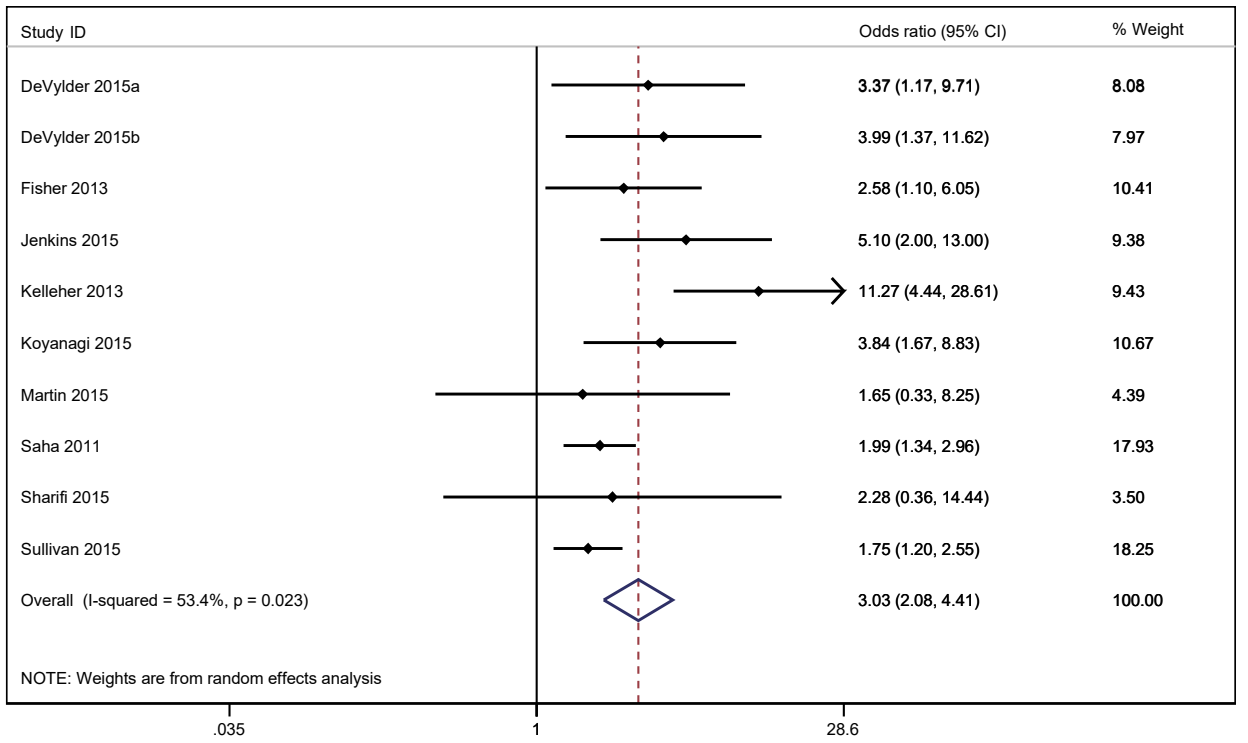

Figure 7: Meta-analysis of the association between psychotic experiences and suicidal behaviour. 


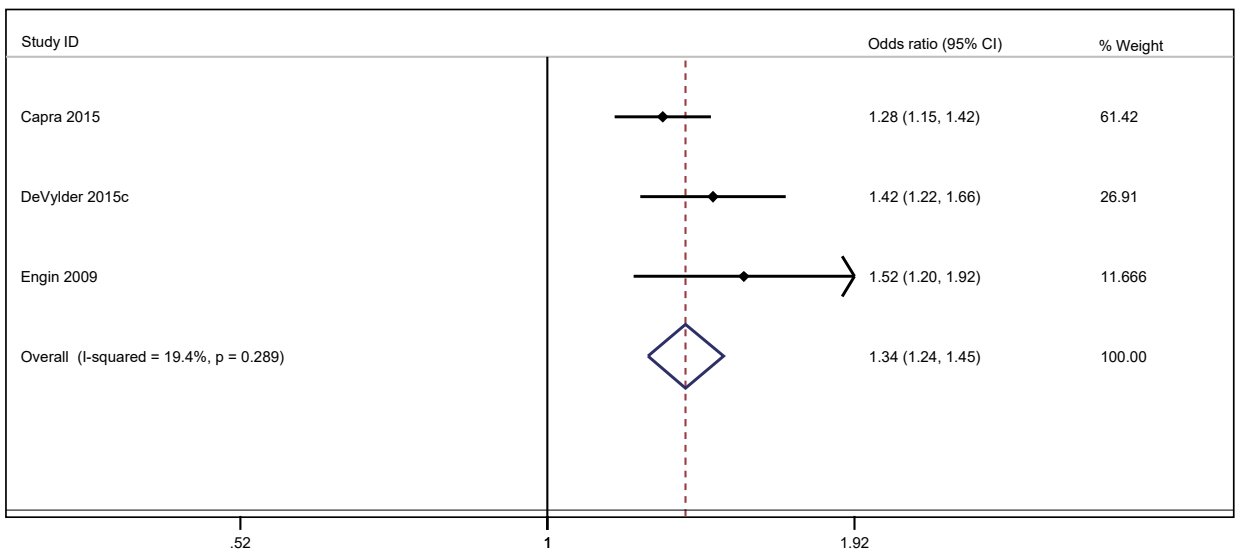

Figure 8: Meta-analysis of the association between psychotic experiences and suicidal behaviour in studies with continuous exposure variables and dichotomous outcome variables.

\section{Adjustment for depression}

The pooled odds ratios for the association between PE and the various outcomes in studies controlling for depression were reduced as compared to non-adjusted metaanalytic estimates, but remained statistically significant for all outcomes except selfharm (Table 2). Only one study assessed the association between PE and self-harm while controlling for depression and found that PE in absence of self-awareness of mental distress and help-seeking behaviours were not associated with self-harm. The pooled odds ratio for the adjusted association between PE and self-injurious behaviour was 2.02 (1.71 - 2.39). Jang et al. (2014) assessed the association between PE and suicidal ideation using a continuous exposure (score on Eppendorf Schizophrenia inventory) and a continuous outcome measure (score on Beck's Scale for Suicidal Ideation) and found a statistically significant association $(\beta=0.44, p<0.0001)$ that greatly decreased in magnitude, but remained statistically significant after controlling for depression $(\beta=0.13$, $\mathrm{p}<0.0001)$. Meta-regression analysis confirmed that effect sizes adjusted for depression were smaller than effect sizes unadjusted for depression (OR=0.51 95\% Cl 0.38 - 0.69). Trim-and-fill procedure decreased the pooled odds ratios of the meta-analyses including studies controlling for depression for all outcomes, indicating possible publication bias, although the results remained statistically significant.

\section{Sensitivity analyses and adjustment}

Odds ratios in the permutation analyses were slightly larger than in original analyses (Table 2). Meta-regression analyses showed that reported adjusted effect sizes are smaller than unadjusted effect sizes ( $O R=0.59,95 \% \mathrm{Cl} 0.44-0.78)$. Reported effect sizes from longitudinal studies were smaller than effect sizes from cross-sectional studies, but this difference was not statistically significant ( $O R=0.94,95 \% \mathrm{Cl} 0.60-1.46)$. A separate 
meta-analysis of the effect sizes unadjusted for depression or other mental disorders from longitudinal studies showed a pooled odds ratio of $4.39(95 \% \mathrm{Cl} 2.14-9.00)$ for the association between PE and suicidal behaviour. The pooled odds ratio decreased to 1.88 (95\% Cl $1.34-2.64)$ after only including longitudinal studies controlling for depression and other mental disorders, representing a reduction in excess risk of $74 \%$.

Table 2: Results of main meta-analyses, adjustment for depression and permutation sensitivity analyses.

\begin{tabular}{llll}
\hline Outcome & $\begin{array}{l}\text { Regular meta-analyses } \\
\text { Pooled OR's }(95 \% \mathrm{Cl}), \mathrm{N}\end{array}$ & $\begin{array}{l}\text { Studies adjusted for depression Permutation } \\
\text { Pooled OR's }(95 \% \mathrm{Cl}), \mathrm{N}\end{array}$ & $\begin{array}{l}\text { } \\
\text { sensitivity analyses } \\
\text { Pooled OR's }(95 \% \mathrm{Cl})\end{array}$ \\
\hline Self-injurious behaviour & $3.20(2.33-4.40), 89869$ & $2.02(2.33-4.40), 73987$ & $3.91(2.64-5.46)$ \\
Self-harm & $2.36(1.13-4.95), 23100$ & $1.60(0.80-3.10), 16131$ & $4.30(1.70-10.17)$ \\
Suicidal thoughts & $2.47(1.71-3.59), 76216$ & $2.00(1.57-2.55), 56827$ & $3.88(2.59-5.54)$ \\
Suicidal behaviour & $3.03(2.08-4.41), 52005$ & $2.10(1.65-2.66), 47217$ & $4.61(2.85-7.09)$ \\
\hline
\end{tabular}

\section{Discussion}

\section{Summary of results}

The present meta-analysis assessed the association between PE and self-injurious behaviour in general population samples using different outcome measures: a) Selfinjurious behaviour (summary outcome measure); b) NSSI; c) suicidal thoughts and d) suicidal behaviour. The main result of the meta-analysis was that experiencing PE is associated with an increased risk of self-injurious behaviour (OR=3.20 95\% Cl $2.33-$ 4.40). This association was confirmed in both cross-sectional as well as longitudinal studies. The pooled odds ratios increased as the severity of the outcome measure increased, ranging from $2.36(95 \% \mathrm{Cl} 1.13-4.95)$ for self-harm to $3.03(95 \% \mathrm{Cl} 2.08-$ 4.41) for suicidal behaviour. Results remained statistically significant after adjusting for various confounders including depression, although adjustment significantly decreased the meta-analytical effect sizes for all outcome measures and the excess risk in longitudinal studies was reduced by $74 \%$. This indicates significant confounding and possibility of residual confounding. Meta-analytical effect sizes from the permutation analyses showed slightly larger meta-analytical effect sizes, demonstrating that the method of selecting the most adjusted effect size for the analyses was conservative.

\section{Interpretation of results}

Even though our results confirmed that PE are strong correlates of self-injurious behaviour, the mechanism behind this association remains unclear. Several possible explanations have been suggested. Studies have identified shared risk factors for PE and selfinjurious behaviour; individuals endorsing PE show a more emotion-oriented coping 
style (Lin et al. 2011), an increased emotional reactivity to stress (Lataster et al. 2009) and deficits in processing speed (Kelleher et al. 2013a), which may also increase the risk of self-injurious behaviour (LeGris and van Reekum 2006). Exposure to traumatic experiences and adverse life events is also associated with an increased risk of both PE and self-injurious behaviour (Wilcox et al. 2012; Iga et al. 2007). Moreover, neuroimaging studies have identified alterations in brain regions that are associated with both PE and suicidality (Jacobson et al. 2010; Koolschijn et al. 2009). It is possible, however, that shared risk factors increase the risk of psychopathology in general, making PE a risk marker between various mental disorders and self-injurious behaviour. The results of the present meta-analysis support this hypothesis.

The finding that adjusted effect sizes were significantly smaller than unadjusted effect sizes, and the finding of substantial reduction of excess risk after adjustment in longitudinal studies, indicates that the association between PE and self-injurious behaviour may be confounded. There is considerable scope for unaccounted residual confounding, as examination of the adjustment method used in the individual studies showed several methodological limitations. Most of the included studies used a crosssectional study design. Besides the fact that causality cannot be inferred from crosssectional studies, it is difficult and conceptually challenging to control for depressive symptoms and other psychopathology using a cross-sectional design, given strong fundamental associations between all psychopathology and increased risk of suicide or selfharm (Cavanagh et al. 2003; Nock et al. 2006). Moreover, several general population surveys have confirmed that approximately $50 \%$ of all individuals with PE have at least one diagnosis for a mental disorder (Jeppesen et al. 2015; van Os et al. 2000; DeVylder et al. 2014; Kelleher et al. 2012a), including depressive or anxiety disorders (van Nierop et al. 2012; Wigman et al. 2012), eating disorder (van Os et al. 2000), substance abuse (Johns et al. 2004), autistic traits (Bevan Jones et al. 2012) and PTSD (Fisher et al. 2013). Indeed, Calkins et al. (2014) showed that experiencing PE is associated with increased odds of a wide variety of psychopathology. This means that any association between PE and self-injurious behaviour is likely to be confounded or mediated by other psychopathology. Controlling for this kind of confounding is methodologically challenging in cross-sectional designs, notwithstanding attempts to do so in several of the included cross-sectional studies, as discussed above. However, others identified other psychopathology as a possible mediator (Kelleher et al. 2012a; Linscott and van Os 2013), while including mediators in a regression model as if they were confounders is not considered valid (Rothman et al. 2008). In sum, because all associations between PE and any included outcome remained statistically significant, the conclusion that PE are associated with self-injurious behaviour including suicidality outcomes is warranted. However, because of suspected residual confounding results should be interpreted tentatively.

Longitudinal study designs have more options to validly control for possible confounders, although it remains similarly difficult to examine how self-injurious behaviour varies in relation to PE whilst keeping other psychopathology constant, when suicidality 
is fundamentally associated with the psychopathology that is being adjusted for. In longitudinal studies, controlling for psychopathology using a dichotomous measure at the most severe level (i.e. presence or absence of disease) similarly leaves room for residual confounding. This kind of confounding can be reduced by using a dimensional, continuous measure of the psychopathology, while none of the longitudinal studies used such an approach. Separate analysis of the longitudinal studies showed that the pooled odds ratio of effect sizes adjusted for psychopathology with a dichotomous measure decreased the magnitude of excess risk by $74 \%$, suggesting confounded associations. Future research using broad dimensional measures of confounding by psychopathology is required.

Several authors suggested that PE should be considered a manifestation of general psychological distress (Saha et al. 2011; Capra et al. 2015; DeVylder et al. 2015a; Martin et al. 2015). In a cross-sectional study, PE without self-awareness of mental distress was still associated with an increased risk of suicidal ideation, but not of self-harm. However, a longitudinal study not adjusted for other psychopathology showed that PE in the absence of psychological distress were not associated with an increased risk of suicide or NSSI (Martin et al. 2015), while PE with psychological distress were associated with an increased risk of NSSI and suicide attempts. This suggests that the association between $P E$ and self-injurious behaviour can be explained by psychological distress. However, the difference between PE with and without distress may also simply reflect differences in PE severity along a several dimensions, e.g. conviction, impact and frequency. Psychological distress without PE was also associated with an increased risk of NSSI and suicide attempts, although effect sizes were smaller.

Various studies have shown that PE can be considered as an indicator of severity of non-psychotic psychopathology (Wigman et al. 2012; Wigman et al. 2014; Perlis et al. 2011; Guloksuz et al. 2015; Kelleher et al. 2012a). It is therefore likely that the association between PE and self-injurious behaviour reflects the greater likelihood of selfinjurious behaviour in more severe states of psychopathology. Therefore, even if there is no direct link between PE and self-injurious behaviour, routine assessment of PE in individuals with non-psychotic psychopathology has potential to reveal passive risk markers of self-injurious behaviour.

\section{Methodological issues}

Several methodological issues were encountered while conducting the present metaanalysis. First, not all of the included studies separated their outcome measures into suicidal ideation and suicide attempts. A study by Kelleher et al. (2012b) assessed the association between PE and suicidal behaviour in two general population samples using a single outcome measure for the whole range of suicidality measures including suicidal ideation and suicide attempts. This study could only be included in the main analysis but not in the sub-analyses. Because this study reported larger effect sizes than most other 
studies, the pooled odds ratio for the summary outcome of self-injurious behaviour was larger than the pooled odds ratios of the sub-analyses. A sensitivity analysis excluding the results of the study by Kelleher and colleagues resulted in an odds ratio of 2.93 (95\% Cl $2.12-4.06)$.

Second, five of the included studies used a continuous exposure and/or outcome measure (Capra et al. 2015; Jang et al. 2014; Kerr and Muehlenkamp 2010; DeVylder et al. 2015b; Engin et al. 2009). Two of these studies could not be included in the quantitative analyses because of their study design (Kerr and Muehlenkamp 2010) or because they were the only study to use a continuous outcome measure (Jang et al. 2014). The other three studies were separately analysed after standardisation of the effect sizes. However, only partial standardisation was possible given the fact that empirical variation of the dependent variable is unknown when meta-analytically pooling results of logistic regression. Results of this sub-analysis pointed in the same direction as the main analysis and other sub-analyses.

Finally, several of the included studies simultaneously adjusted for multiple possible confounders, making it difficult to assess the influence of a single confounder, for example depression. Therefore, the most adjusted effect size was selected for the regular meta-analyses, and separate analyses were done for studies adjusting for depression.

\section{Future research}

Longitudinal research is required to determine to what degree any association between PE and self-injurious behaviour exists that is specific and unconfounded, and to examine to what degree the association reflects severity of psychopathology, of which PE are an indicator. A general population sample stratified by the presence or absence of mental disorder and suicidality at baseline may form a valid initial starting point of analysis. In order to minimize residual confounding, analyses should be adjusted for dimensional psychopathology. 


\section{References}

American Psychiatric Association 2013. Diagnostic and Statistical Manual of Mental Disorders (DSM-5), Washington, American Psychiatric Association.

Anderson, J., Huppert, F. \& Rose, G. 1993. Normality, deviance and minor psychiatric morbidity in the community. A population-based approach to General Health Questionnaire data in the Health and Lifestyle Survey. Psychol Med, 23, 475-85.

Bevan Jones, R., Thapar, A., Lewis, G. \& Zammit, S. 2012. The association between early autistic traits and psychotic experiences in adolescence. Schizophr Res, 135, 164-9.

Calkins, M. E., Moore, T. M., Merikangas, K. R., Burstein, M., Satterthwaite, T. D., Bilker, W. B., Ruparel, K., Chiavacci, R., Wolf, D. H., Mentch, F., Qiu, H., Connolly, J. J., Sleiman, P. A., Hakonarson, H., Gur, R. C. \& Gur, R. E. 2014. The psychosis spectrum in a young U.S. community sample: findings from the Philadelphia Neurodevelopmental Cohort. World Psychiatry, 13, 296-305.

Capra, C., Kavanagh, D. J., Hides, L. \& Scott, J. G. 2015. Subtypes of psychotic-like experiences are differentially associated with suicidal ideation, plans and attempts in young adults. Psychiatry Res, 228, 894-8.

Cavanagh, J. T., Carson, A. J., Sharpe, M. \& Lawrie, S. M. 2003. Psychological autopsy studies of suicide: a systematic review. Psychol Med, 33, 395-405.

Devylder, J. E., Burnette, D. \& Yang, L. H. 2014. Co-occurrence of psychotic experiences and common mental health conditions across four racially and ethnically diverse population samples. Psychol Med, 44, 3503-13.

Devylder, J. E. \& Hilimire, M. R. 2015. Suicide Risk, Stress Sensitivity, and Self-Esteem among Young Adults Reporting Auditory Hallucinations. Health Soc Work, 40, 175-81.

Devylder, J. E., Lukens, E. P., Link, B. G. \& Lieberman, J. A. 2015a. Suicidal ideation and suicide attempts among adults with psychotic experiences: data from the Collaborative Psychiatric Epidemiology Surveys. JAMA Psychiatry, 72, 219-25.

Devylder, J. E., Thompson, E., Reeves, G. \& Schiffman, J. 2015b. Psychotic experiences as indicators of suicidal ideation in a non-clinical college sample. Psychiatry Res, 226, 489-93.

Earl, T. R., Fortuna, L. R., Gao, S., Williams, D. R., Neighbors, H., Takeuchi, D. \& Alegria, M. 2015. An exploration of how psychotic-like symptoms are experienced, endorsed, and understood from the National Latino and Asian American Study and National Survey of American Life. Ethn Health, 20, 273-92.

Engin, E., Gurkan, A., Dulgerler, S. \& Arabaci, L. B. 2009. University students' suicidal thoughts and influencing factors. J Psychiatr Ment Health Nurs, 16, 343-54.

Fisher, H. L., Caspi, A., Poulton, R., Meier, M. H., Houts, R., Harrington, H., Arseneault, L. \& Moffitt, T. E. 2013. Specificity of childhood psychotic symptoms for predicting schizophrenia by 38 years of age: a birth cohort study. Psychol Med, 43, 2077-86.

Guloksuz, S., Van Nierop, M., Lieb, R., Van Winkel, R., Wittchen, H. U. \& Van Os, J. 2015. Evidence that the presence of psychosis in non-psychotic disorder is environment-dependent and mediated by severity of non-psychotic psychopathology. Psychol Med, 45, 2389-401.

Hamza, C. A., Stewart, S. L. \& Willoughby, T. 2012. Examining the link between nonsuicidal self-injury and suicidal behavior: a review of the literature and an integrated model. Clin Psychol Rev, 32, 482-95.

Hawton, K. \& Van Heeringen, K. 2009. Suicide. Lancet, 373, 1372-81.

Hawton, K., Saunders, K. E. \& O'connor, R. C. 2012. Self-harm and suicide in adolescents. Lancet, 379, $2373-82$.

Iga, J., Ueno, S., Yamauchi, K., Numata, S., Tayoshi-Shibuya, S., Kinouchi, S., Nakataki, M., Song, H., Hokoishi, K., Tanabe, H., Sano, A. \& Ohmori, T. 2007. The Val66Met polymorphism of the brain-derived neurotrophic factor gene is associated with psychotic feature and suicidal behavior in Japanese major depressive patients. Am J Med Genet B Neuropsychiatr Genet, 144B, 1003-6.

Jacobson, S., Kelleher, I., Harley, M., Murtagh, A., Clarke, M., Blanchard, M., Connolly, C., O'hanlon, E., Garavan, H. \& Cannon, M. 2010. Structural and functional brain correlates of subclinical psychotic symptoms in 11-13 year old schoolchildren. Neuroimage, 49, 1875-85.

Jang, J. H., Lee, Y. J., Cho, S. J., Cho, I. H., Shin, N. Y. \& Kim, S. J. 2014. Psychotic-like experiences and their relationship to suicidal ideation in adolescents. Psychiatry Res, 215, 641-5. 
Jenkins, R., Othieno, C., Omollo, R., Ongeri, L., Sifuna, P., Ongecha, M., Mboroki, J. K., Kiima, D. \& Ogutu, B. 2015. Tedium vitae, death wishes, suicidal ideation and attempts in Kenya-prevalence and risk factors. BMC Public Health, 15, 759.

Jeppesen, P., Clemmensen, L., Munkholm, A., Rimvall, M. K., Rask, C. U., Jorgensen, T., Larsen, J. T., Petersen, L., Van Os, J. \& Skovgaard, A. M. 2015. Psychotic experiences co-occur with sleep problems, negative affect and mental disorders in preadolescence. J Child Psychol Psychiatry, 56, 558-65.

Johns, L. C., Cannon, M., Singleton, N., Murray, R. M., Farrell, M., Brugha, T., Bebbington, P., Jenkins, R. \& Meltzer, H. 2004. Prevalence and correlates of self-reported psychotic symptoms in the British population. Br J Psychiatry, 185, 298-305.

Katz, M. H. 2006. Multivariable Analysis: A Practical Guide for Clinicians, Cambridge University Press.

Kaymaz, N., Drukker, M., Lieb, R., Wittchen, H. U., Werbeloff, N., Weiser, M., Lataster, T. \& Van Os, J. 2012. Do subthreshold psychotic experiences predict clinical outcomes in unselected non-help-seeking populationbased samples? A systematic review and meta-analysis, enriched with new results. Psychol Med, 42, 2239-53.

Kelleher, I., Keeley, H., Corcoran, P., Lynch, F., Fitzpatrick, C., Devlin, N., Molloy, C., Roddy, S., Clarke, M. C., Harley, M., Arseneault, L., Wasserman, C., Carli, V., Sarchiapone, M., Hoven, C., Wasserman, D. \& Cannon, M. 2012a. Clinicopathological significance of psychotic experiences in non-psychotic young people: evidence from four population-based studies. Br J Psychiatry, 201, 26-32.

Kelleher, I., Lynch, F., Harley, M., Molloy, C., Roddy, S., Fitzpatrick, C. \& Cannon, M. 2012b. Psychotic symptoms in adolescence index risk for suicidal behavior: findings from 2 population-based case-control clinical interview studies. Arch Gen Psychiatry, 69, 1277-83.

Kelleher, I., Clarke, M. C., Rawdon, C., Murphy, J. \& Cannon, M. 2013a. Neurocognition in the extended psychosis phenotype: performance of a community sample of adolescents with psychotic symptoms on the MATRICS neurocognitive battery. Schizophr Bull, 39, 1018-26.

Kelleher, I., Corcoran, P., Keeley, H., Wigman, J. T., Devlin, N., Ramsay, H., Wasserman, C., Carli, V., Sarchiapone, M., Hoven, C., Wasserman, D. \& Cannon, M. 2013b. Psychotic symptoms and population risk for suicide attempt: a prospective cohort study. JAMA Psychiatry, 70, 940-8.

Kendler, K. S. \& Gardner, C. O., Jr. 1998. Boundaries of major depression: an evaluation of DSM-IV criteria. Am J Psychiatry, 155, 172-7.

Kerr, P. L. \& Muehlenkamp, J. J. 2010. Features of psychopathology in self-injuring female college students. Journal of Mental Health Counseling, 32, 290-308.

Kessler, R. C., Berglund, P., Borges, G., Nock, M. \& Wang, P. S. 2005. Trends in suicide ideation, plans, gestures, and attempts in the United States, 1990-1992 to 2001-2003. JAMA, 293, 2487-95.

Koolschijn, P. C., Van Haren, N. E., Lensvelt-Mulders, G. J., Hulshoff Pol, H. E. \& Kahn, R. S. 2009. Brain volume abnormalities in major depressive disorder: a meta-analysis of magnetic resonance imaging studies. Hum Brain Mapp, 30, 3719-35.

Koyanagi, A., Stickley, A. \& Haro, J. M. 2015. Subclinical psychosis and suicidal behavior in England: Findings from the 2007 Adult Psychiatric Morbidity Survey. Schizophr Res, 168, 62-7.

Lataster, T., Wichers, M., Jacobs, N., Mengelers, R., Derom, C., Thiery, E., Van Os, J. \& Myin-Germeys, I. 2009. Does reactivity to stress cosegregate with subclinical psychosis? A general population twin study. Acta Psychiatr Scand, 119, 45-53.

Legris, J. \& Van Reekum, R. 2006. The neuropsychological correlates of borderline personality disorder and suicidal behaviour. Can J Psychiatry, 51, 131-42.

Lewis-Fernandez, R., Horvitz-Lennon, M., Blanco, C., Guarnaccia, P. J., Cao, Z. \& Alegria, M. 2009. Significance of endorsement of psychotic symptoms by US Latinos. J Nerv Ment Dis, 197, 337-47.

Lin, A., Wigman, J. T., Nelson, B., Vollebergh, W. A., Van Os, J., Baksheev, G., Ryan, J., Raaijmakers, Q. A., Thompson, A. \& Yung, A. R. 2011. The relationship between coping and subclinical psychotic experiences in adolescents from the general population--a longitudinal study. Psychol Med, 41, 2535-46.

Linscott, R. J. \& Van Os, J. 2013. An updated and conservative systematic review and meta-analysis of epidemiological evidence on psychotic experiences in children and adults: on the pathway from proneness to persistence to dimensional expression across mental disorders. Psychol Med, 43, 1133-49. 
Martin, G., Thomas, H., Andrews, T., Hasking, P. \& Scott, J. G. 2015. Psychotic experiences and psychological distress predict contemporaneous and future non-suicidal self-injury and suicide attempts in a sample of Australian school-based adolescents. Psychol Med, 45, 429-37.

Menard, S. 2004. Six Approaches to Calculating Standardized Logistic Regression Coefficients. The American Statistician, 58, 218-223.

Miller, G. A. \& Chapman, J. P. 2001. Misunderstanding analysis of covariance. J Abnorm Psychol, 110, 40-8.

Nishida, A., Sasaki, T., Nishimura, Y., Tanii, H., Hara, N., Inoue, K., Yamada, T., Takami, T., Shimodera, S., Itokawa, M., Asukai, N. \& Okazaki, Y. 2010. Psychotic-like experiences are associated with suicidal feelings and deliberate self-harm behaviors in adolescents aged 12-15 years. Acta Psychiatr Scand, 121, 301-7.

Nishida, A., Shimodera, S., Sasaki, T., Richards, M., Hatch, S. L., Yamasaki, S., Usami, S., Ando, S., Asukai, N. \& Okazaki, Y. 2014. Risk for suicidal problems in poor-help-seeking adolescents with psychotic-like experiences: findings from a cross-sectional survey of 16,131 adolescents. Schizophr Res, 159, 257-62.

Nock, M. K., Joiner, T. E., Jr., Gordon, K. H., Lloyd-Richardson, E. \& Prinstein, M. J. 2006. Non-suicidal self-injury among adolescents: diagnostic correlates and relation to suicide attempts. Psychiatry Res, 144, 65-72.

Nock, M. K. 2010. Self-injury. Annu Rev Clin Psychol, 6, 339-63.

Olfson, M., Weissman, M. M., Leon, A. C., Farber, L. \& Sheehan, D. V. 1996. Psychotic symptoms in primary care. J Fam Pract, 43, 481-8.

Olfson, M., Lewis-Fernandez, R., Weissman, M. M., Feder, A., Gameroff, M. J., Pilowsky, D. \& Fuentes, M. 2002. Psychotic symptoms in an urban general medicine practice. Am J Psychiatry, 159, 1412-9.

Ostling, S. \& Skoog, I. 2002. Psychotic symptoms and paranoid ideation in a nondemented population-based sample of the very old. Arch Gen Psychiatry, 59, 53-9.

Perala, J., Suvisaari, J., Saarni, S. I., Kuoppasalmi, K., Isometsa, E., Pirkola, S., Partonen, T., Tuulio-Henriksson, A., Hintikka, J., Kieseppa, T., Harkanen, T., Koskinen, S. \& Lonnqvist, J. 2007. Lifetime prevalence of psychotic and bipolar I disorders in a general population. Arch Gen Psychiatry, 64, 19-28.

Perlis, R. H., Uher, R., Ostacher, M., Goldberg, J. F., Trivedi, M. H., Rush, A. J. \& Fava, M. 2011. Association between bipolar spectrum features and treatment outcomes in outpatients with major depressive disorder. Arch Gen Psychiatry, 68, 351-60.

Polanczyk, G., Moffitt, T. E., Arseneault, L., Cannon, M., Ambler, A., Keefe, R. S., Houts, R., Odgers, C. L. \& Caspi, A. 2010. Etiological and clinical features of childhood psychotic symptoms: results from a birth cohort. Arch Gen Psychiatry, 67, 328-38.

Rothman, K. J., Greenland, S. \& Lash, T. L. 2008. Modern Epidemiology, Wolters Kluwer Health/Lippincott Williams \& Wilkins.

Saha, S., Scott, J. G., Johnston, A. K., Slade, T. N., Varghese, D., Carter, G. L. \& Mcgrath, J. J. 2011. The association between delusional-like experiences and suicidal thoughts and behaviour. Schizophr Res, 132, 197-202.

Sharifi, V., Eaton, W. W., Wu, L. T., Roth, K. B., Burchett, B. M. \& Mojtabai, R. 2015. Psychotic experiences and risk of death in the general population: 24-27 year follow-up of the Epidemiologic Catchment Area study. Br J Psychiatry, 207, 30-6.

Sullivan, S. A., Lewis, G., Gunnell, D., Cannon, M., Mars, B. \& Zammit, S. 2015. The longitudinal association between psychotic experiences, depression and suicidal behaviour in a population sample of adolescents. Soc Psychiatry Psychiatr Epidemiol, 50, 1809-17.

Sveticic, J. \& De Leo, D. 2012. The hypothesis of a continuum in suicidality: a discussion on its validity and practical implications. Ment IIIn, 4, e15.

Temmingh, H., Stein, D. J., Seedat, S. \& Williams, D. R. 2011. The prevalence and correlates of hallucinations in a general population sample: findings from the South African Stress and Health Study. Afr J Psychiatry (Johannesbg), 14, 211-7.

Van Nierop, M., Van Os, J., Gunther, N., Myin-Germeys, I., De Graaf, R., Ten Have, M., Van Dorsselaer, S., Bak, M. \& Van Winkel, R. 2012. Phenotypically continuous with clinical psychosis, discontinuous in need for care: evidence for an extended psychosis phenotype. Schizophr Bull, 38, 231-8.

Van Os, J., Hanssen, M., Bijl, R. \& Ravelli, A. 2000. Straus (1969) revisited: A psychosis continuum in the general population? Schizophrenia Research, 45, 11-20. 
Van Os, J., Linscott, R. J., Myin-Germeys, I., Delespaul, P. \& Krabbendam, L. 2009. A systematic review and meta-analysis of the psychosis continuum: evidence for a psychosis proneness-persistence-impairment model of psychotic disorder. Psychol Med, 39, 179-95.

Weiner, I. B., Schinka, J. A. \& Velicer, W. F. 2003. Handbook of Psychology, John Wiley \& Sons.

Wigman, J. T., Van Nierop, M., Vollebergh, W. A., Lieb, R., Beesdo-Baum, K., Wittchen, H. U. \& Van Os, J. 2012. Evidence that psychotic symptoms are prevalent in disorders of anxiety and depression, impacting on illness onset, risk, and severity--implications for diagnosis and ultra-high risk research. Schizophr Bull, 38, 247-57.

Wigman, J. T., Van Os, J., Abidi, L., Huibers, M. J., Roelofs, J., Arntz, A., Kelleher, I. \& Peeters, F. P. 2014. Subclinical psychotic experiences and bipolar spectrum features in depression: association with outcome of psychotherapy. Psychol Med, 44, 325-36.

Wilcox, H. C., Arria, A. M., Caldeira, K. M., Vincent, K. B., Pinchevsky, G. M. \& O'grady, K. E. 2012. Longitudinal predictors of past-year non-suicidal self-injury and motives among college students. Psychol Med, 42, 717-26.

World Health Organization 2014. Preventing suicide: A global imperative, Geneva, World Health Organization. 



\section{Chapter}

\section{Psychotic experiences and incident suicidal ideation and behaviour: disentangling the longitudinal associations from connected psychopathology}

Steven Honings ${ }^{1}$, Marjan Drukker ${ }^{1}$, Martine van Nierop ${ }^{2}$, Ruud van Winkel ${ }^{2,3}$, HansUlrich Wittchen ${ }^{4,5}$, Roselind Lieb ${ }^{5,6}$, Margreet ten Have ${ }^{7}$, Ron de Graaf ${ }^{7}$, Saskia van Dorsselaer ${ }^{7}$, Jim van Os ${ }^{1,8,9}$

1. Department of Psychiatry and Psychology, South Limburg Mental Health Research and Teaching Network, Maastricht University Medical Centre, Maastricht, the Netherlands.

2. Katholieke Universiteit Leuven, Department of Neuroscience, Research Group Psychiatry, Centre for Contextual Psychiatry, Leuven, Belgium.

3. University Psychiatric Center Katholieke Universiteit Leuven, Campus Kortenberg, Leuvensesteenweg, Kortenberg, Belgium.

4. Institute of Clinical Psychology and Psychotherapy, Technische Universität Dresden, Dresden, Germany.

5. Max Planck Institute of Psychiatry, Munich, Germany.

6. Department of Psychology, Division of Clinical Psychology and Epidemiology, University of Basel, Basel, Switzerland.

7. Netherlands Institute of Mental Health and Addiction, Utrecht, the Netherlands

8. King's College London, King's Health Partners, Department of Psychosis Studies, Institute of Psychiatry, London, UK

9. Department of Psychiatry, Brain Center Rudolf Magnus Institute, University Medical Center Utrecht, Utrecht, The Netherlands.

Published as:

Honings, S., Drukker, M., Van Nierop, M., Van Winkel, R., Wittchen, H. U., Lieb, R., Ten Have, M., De Graaf, R., Van Dorsselaer, S. \& Van Os, J. 2016. Psychotic experiences and incident suicidal ideation and behaviour: Disentangling the longitudinal associations from connected psychopathology. Psychiatry Res, 245, 267-275. 


\begin{abstract}
Background: This study examines the longitudinal associations between psychotic experiences (PE) and incident suicidal ideation and behaviour in the general population, and to what degree the association may be confounded by non-psychotic psychopathology. Method: Data from three prospective, general population cohorts were combined into one dataset $(n=15837)$ and analysed using logistic regression, controlling for continuous measures of depression, anxiety and mania symptoms. Analyses were conducted in the entire sample, and in subsamples stratified by presence or absence of mental disorders. Results: The presence of PE at baseline increased the risk of incident suicidal ideation and behaviour. However, adjustment for dimensional measures of psychopathology reduced effect sizes, although PE remained significantly associated with suicide attempts. Further examination of the associations revealed that PE were only associated with suicide attempts in individuals with at least one mental disorder. Similarly, in individuals without mental disorders, the risk of suicidal ideation increased as PE cooccurred with more symptom domains.

Conclusion: The results of this study confirm that individuals with PE are at increased risk of suicidal ideation and behaviour. However, these associations are not specific, but reflect the increased risk of suicidal ideation in individuals with subthreshold multidimensional psychopathology and suicide attempts in individuals with co-occurring mental disorders.
\end{abstract}




\section{Introduction}

The presence of low grade psychotic experiences (PE) in the general population has been associated with an increased risk of suicidal ideation and behaviour (Honings et al. 2016). It remains unclear, however, how to isolate this specific association from the association between non-psychotic psychopathology and suicidal ideation and behaviour, since non-psychotic psychopathology is strongly connected to both PE (Jeppesen et al. 2015; van Os et al. 2000; DeVylder et al. 2014; Kelleher et al. 2012b; Guloksuz et al. 2015; Kelleher et al. 2012a), and suicidal behaviour (Cavanagh et al. 2003; ten Have et al. 2013; ten Have et al. 2009).

Suicidality comprises various phenomena on a continuum ranging from suicidal ideation, suicide plans and suicide attempts to completed suicide (Sveticic and De Leo 2012; Nock 2010). Since suicide and suicide attempts are highly prevalent, identification of causal risk factors is urgently required, as knowledge in this area may assist in reducing the number of suicidal acts (World Health Organization 2014; Hawton and van Heeringen 2009; Nock et al. 2008). Even though the mechanisms underlying suicidal behaviour remain poorly understood, research has shown that it is likely determined by a complex interplay of biological, psychological, environmental and cultural factors (World Health Organization 2014; O'Connor et al. 2011). Mental disorders are an important risk factor for suicidal behaviour (Palmer et al. 2005; Nordentoft et al. 2011; Bostwick and Pankratz 2000): of the individuals who committed suicide, $91 \%$ had a mental disorder, most commonly a depressive disorder, substance abuse or schizophrenia (Cavanagh et al. 2003; ten Have et al. 2013; ten Have et al. 2009). Recently, PE have been identified as a risk factor for suicidal ideation and behaviour in general population individuals (Honings et al. 2016). A similar association has been reported for individuals at risk for psychosis (Taylor et al. 2015).

PE refer to delusional or hallucinatory experiences below the threshold of a diagnosable psychotic disorder, often in absence of significant psychological distress (Linscott and van Os 2013; Kelleher et al. 2012a). General population surveys have shown that PE are highly prevalent in the general population, with a meta-analytical prevalence of 7.2\% (Linscott and van Os 2013; Kelleher et al. 2012a). PE were identified as a risk factor for several mental health outcomes, including both psychotic (Linscott and van Os 2013; Kaymaz et al. 2012) and non-psychotic psychopathology (Kelleher et al. 2012b), and are associated with increased treatment use and need for care in general population samples (Murphy et al. 2012). Moreover, PE serve as an indicator of illness severity and poor outcome in persons with non-psychotic psychopathology (Wigman et al. 2012; Wigman et al. 2014; Perlis et al. 2011; Guloksuz et al. 2015; Kelleher et al. 2012b)

A recent meta-analysis reported that general population individuals with PE had an increased risk of both suicidal thoughts (i.e. suicidal ideation or suicidal plans) and suicidal behaviour (i.e. suicidal attempts or completed suicide) compared with individuals 
without PE. Pooled odds ratios (OR) were 2.47 (95\% Cl 1.71 - 3.59; suicidal thoughts) and 3.03 (95\% Cl 2.08 - 4.41; suicidal behaviour), respectively (Honings et al. 2016). However, the association between PE and suicidal ideation and behaviour is confounded, since adjustment for non-psychotic psychopathology in longitudinal studies resulted in a $74 \%$ excess risk reduction (Honings et al. 2016).

At least half of all individuals with PE have a diagnosable mental disorder (Jeppesen et al. 2015; van Os et al. 2000; DeVylder et al. 2014; Kelleher et al. 2012b). Moreover, individuals with PE without a mental disorder are at increased risk to develop one (Linscott and van Os 2013; Kelleher et al. 2012b; Kaymaz et al. 2012). Therefore, it remains unclear whether there is a specific association between PE and suicidal behaviour, or whether the association reflects the increased risk of suicidal behaviour in more severe states of mental distress associated with multidimensional psychopathology (van Nierop et al. 2015b; Guloksuz et al. 2015). In a previous study, PE were only associated with suicide attempts in individuals with associated psychological distress, while in the absence of psychological distress there was no association (Martin et al. 2015). Similarly, PE were particularly predictive of suicidal behaviour in the context of a mental disorder (DeVylder et al. 2015b; Kelleher et al. 2013). Recent cross-sectional studies similarly provided evidence that the link between PE and suicidal behaviour is not specific, and can be explained by psychosocial factors and shared common causes that contribute to cumulative stress (DeVylder et al. 2015a; Jahn et al. 2016).

Previous studies had some methodological limitations. First, the association between PE and suicidal ideation and behaviour has been investigated mostly in crosssectional studies (Capra et al. 2015; DeVylder et al. 2015b; Jang et al. 2014; Kelleher et al. 2012c; Nishida et al. 2010; Saha et al. 2011; Temmingh et al. 2011), disabling causal inferences. Second, several studies included psychiatric diagnoses in the regression models to control for confounding (Calkins et al. 2014; DeVylder et al. 2015b; Fisher et al. 2013; Koyanagi et al. 2015; Lewis-Fernandez et al. 2009; Olfson et al. 2002; Saha et al. 2011; Sharifi et al. 2015). However, it is conceptually impossible to examine a confounder in the association between PE and suicidal behaviour when that confounder, for example depression, is fundamentally associated with the outcome (Miller and Chapman 2001). Moreover, the addition of a dichotomous confounder to a regression model (i.e. presence or absence of a diagnosable disorder) leaves room for residual confounding, while the use of a continuous, dimensional estimate of psychopathology can prevent that.

The present study aims to examine the longitudinal associations between PE and suicidal ideation and behaviour in a dataset combining three different prospective, general population cohorts. In addition, it was attempted to disentangle the associations between PE and suicidal ideation and behaviour from the associations between cooccurring non-psychotic psychopathology and suicidality, both at the level of mental disorders and at the level of dimensional psychopathology. Furthermore, methodologi- 
cal limitations associated with earlier analyses were addressed by using three prospective cohort studies, stratified by presence or absence of various mental disorders.

The hypothesis was that the associations between PE and suicidal ideation and behaviour reflect the increased risk of these outcomes in participants with more severe mental distress in the context of mental disorders or multidimensional psychopathology falling under the threshold of a mental disorder. If this is the case, the associations between PE and suicidal ideation and behaviour would only exist in participants with a mental disorder or with high levels of psychopathology. Thus, PE in the absence of either a mental disorder or high levels of sub-disorder psychopathology were hypothesized to not display any associations with suicidal ideation and behaviour.

\section{Methods}

\section{Samples and design}

This study uses data from three different prospective-longitudinal community studies: the Early Developmental Stages of Psychopathology study (EDSP) and the first and second Netherlands Mental Health Survey and Incidence Studies (NEMESIS and NEMESIS-2).

The EDSP is a prospective study in the German general population. A random sample of all residents was drawn from the population registers to mirror the distribution of 14 to 24-year-olds in Munich. Detailed information about the study characteristics including the instruments and procedures used can be found elsewhere (Lieb et al. 2000; Wittchen et al. 1998). The study consisted of a baseline survey $(\mathrm{TO}, n=3021)$ and three follow-up assessments (T1, T2, T3). Because PE was first enquired at T2, only waves T2 and T3 were used. At T2, the average age of participants was 21.7 years ( $S D=3.4)$. The mean follow-up time between $\mathrm{T} 2$ and $\mathrm{T} 3$ was 4.8 years $(\mathrm{SD}=0.7)$. Response rates were $84 \%$ at T2 $(n=2548)$ and $73 \%$ at T3 $(n=2210)$.

NEMESIS, and its successor NEMESIS-2, are longitudinal cohort studies of the prevalence, incidence, course and consequences of mental disorders in the Dutch general population. Both studies applied multistage, random sampling procedures of municipalities and households. Detailed information about the study characteristics was published elsewhere (Bijl et al. 1998; de Graaf et al. 2010). NEMESIS included 7076 participants aged 18-64 years (average age at baseline 41.2 years, $S D=12.2$ ) and consisted of two follow-up surveys, respectively one (T1) and three (T2) years after the baseline measurement (T0). At T2, a total of 4796 persons participated (response rate 68\%). NEMESIS-2 included 6646 participants aged 18-65 years (average age at baseline 44.2 years, SD=12.5). Three years after baseline (T0), 5303 persons participated in the follow-up assessment (T1, response rate 80\%). For the present analysis, waves T0, T1 and T2 from NEMESIS and waves TO and T1 from NEMESIS-2 were used, both covering a time period of three years. 
A flowchart visualizing the inclusion, exclusion and attrition of participants is shown in Figure 1.

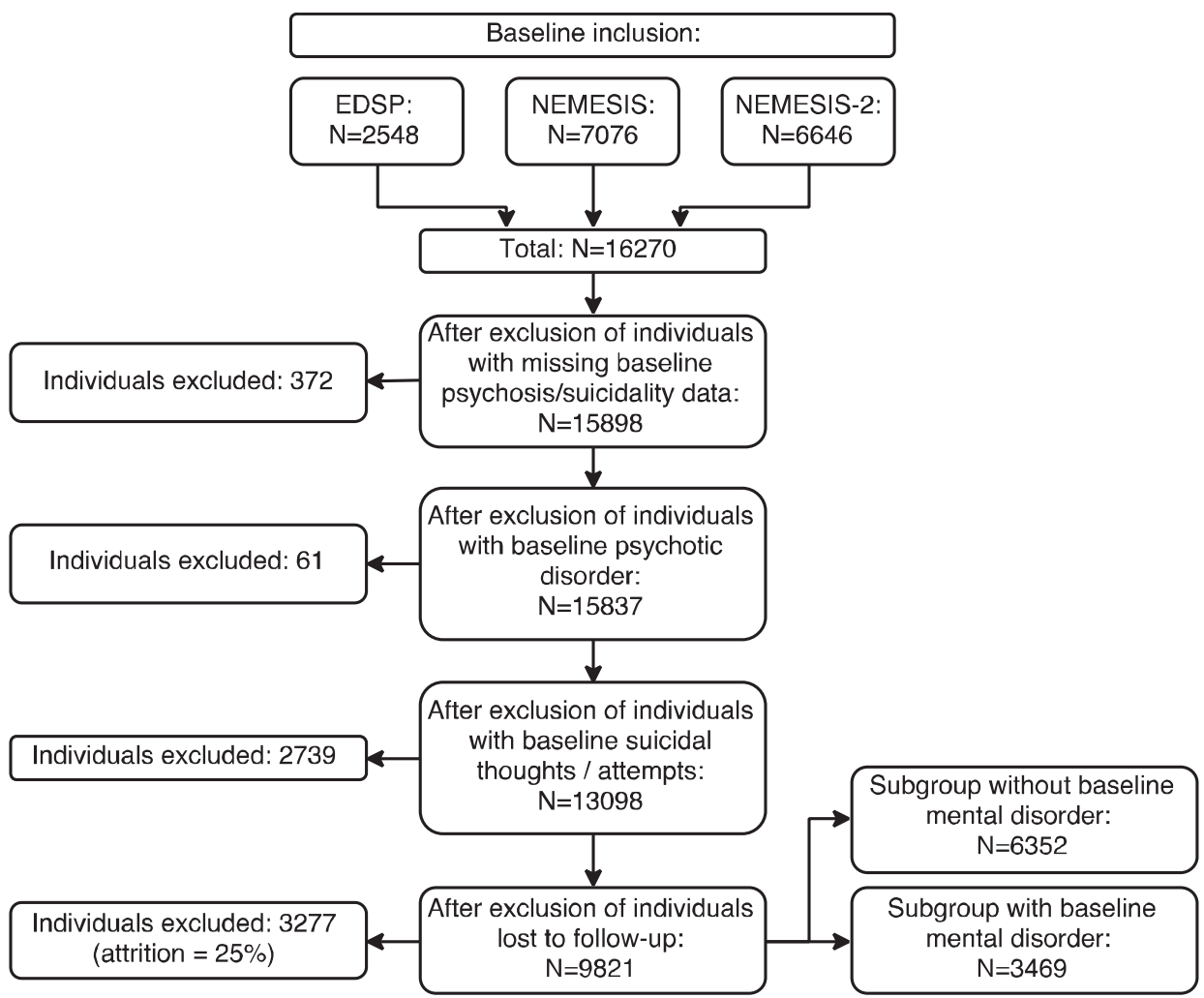

Figure 1: Flowchart of inclusion, exclusion and attrition of participants.

\section{Diagnostic instruments}

In all three studies, participants were interviewed using various versions of the Composite International Diagnostic Interview (CIDI) (World Health Organization 1990). This is a comprehensive and standardized diagnostic interview assessing symptoms, syndromes and diagnoses of mental disorders according to the diagnostic criteria of a version of the Diagnostic and Statistical Manual of Mental disorders (DSM). The instrument is designed to be used by trained lay interviewers, who read questions in a standardized way and record participants' answers. Therefore, the CIDI is essentially a self-report instrument (Eaton et al. 2000). Both the validity (Reed et al. 1998; Haro et al. 2006) and the test-retest reliability have been established, showing that the CIDI provides valid diagnoses for almost all non-psychotic disorders with good to excellent kappa coefficients for most diagnostic sections (Wittchen 1994). 
In the EDSP, a computerized version of the Munich-Composite International Diagnostic Interview (M-CIDI) (Wittchen and Pfister 1997) was used. This instrument is based on the CIDI version 1.2 (World Health Organization 1990), and assesses symptoms and diagnoses according to the third revised edition of the Diagnostic and Statistical Manual of Mental disorders (DSM-III-R). Interviews were conducted by trained psychologists. In NEMESIS, interviews were performed using the CIDI version 1.1 by trained lay interviewers (Smeets and Dingemans 1993). Like the M-CIDI, this version of the CIDI generated DSM-III-R diagnoses. Participants of the NEMESIS-2 were interviewed at home by trained interviewers using the CIDI version 3.0 (Alonso et al. 2004; de Graaf et al. 2008). This version of the CIDI generated DSM-IV diagnoses, and contained a screening section with key questions for most mental disorders (de Graaf et al. 2010). Only participants answering positively on a key question were administered the complete disorder section.

For reasons of consistency across studies, only baseline lifetime diagnoses of any anxiety disorder, major depressive episode, dysthymia, any substance use disorder and bipolar disorder were used in the analyses.

\section{Assessment of psychotic experiences}

In the EDSP, PE were first assessed at T2 using the G section of the M-CIDI. This section included 14 items on delusions and 5 items on hallucinations corresponding to classic psychotic symptoms like persecution, various hallucinations and thought interference. The interviews were conducted by trained psychologists who were allowed to probe with follow-up clinical questions. For the present analysis, T2 was used as baseline. In NEMESIS, the G section of the CIDI version 1.1 was used to assess PE. This section consists of 13 items on delusions and 4 items on hallucinations, corresponding with the EDSP items. In NEMESIS-2, a psychosis add-on instrument based on the $\mathrm{G}$ section of the previous CIDI versions was included. This add-on instrument consists of 20 psychotic symptoms corresponding to the symptoms assessed in the NEMESIS and EDSP. Detailed descriptions of the specific PE items can be found in previous work using EDSP (van Rossum et al. 2011), NEMESIS (Smeets et al. 2013) and NEMESIS-2 (van Nierop et al. 2012).

In both the NEMESIS and NEMESIS-2, an experienced clinician did a follow-up telephone interview when participants reported a psychotic symptom to assess whether this symptom was a true PE, using questions from the Structured Clinical Interview for DSM-III-R or DSM-IV, respectively. In NEMESIS, the proportion of participants eligible for a telephone interview that was actually re-interviewed was $47 \%$. The CIDI rating was corrected to match clinical follow-up interview where possible. Re-interview resulted in a change in $6.9 \%$ of the ratings (van Os et al. 2001). In NEMESIS-2, 74\% of all eligible participants was re-assessed. From the NEMESIS-2 data, only clinically validated PE were used in the analyses. 
For the present analysis, PE were dichotomised. Thus, presence of PE was defined as at least one CIDI psychotic symptom, consistent with previous work in EDSP, NEMESIS and NEMESIS-2 (Gevonden et al. 2014; Cougnard et al. 2007; van Os et al. 2000; van Os et al. 2001). At baseline, lifetime prevalence of PE was assessed.

\section{Assessment of suicidal ideation and behaviour}

The outcome measures in the present study were incident suicidal ideation (hereafter suicidal ideation) and incident suicide attempts (hereafter suicide attempts). In EDSP and NEMESIS, suicidal ideation and suicide attempts were assessed using the following questions from the CIDI depression section: a) Have you had a period of two weeks or more during which you wanted to be dead? b) Have you been so down that you thought of committing suicide? c) Have you attempted suicide? In NEMESIS-2, the suicidality questions in the depression section were slightly different: a) Have you thought of committing suicide? b) Have you made plans to end your life? c) Have you attempted suicide? In NEMESIS-2, these questions were only asked to participants who answered positively to the stem questions of the depression section. Therefore, to prevent false negatives in participants with suicidal ideation or suicide attempts outside the context of depression, three additional items from the suicidality section of the CIDI version 3.0 were used. In this section, all participants are asked about the following three events: a) Have you seriously thought about committing suicide? b) Have you made a plan for committing suicide? c) Have you attempted suicide? In all three studies, questions referred to any incident of suicidal ideation or suicide attempt since the baseline assessment.

For the present analysis, incident suicidal ideation was defined as a positive answer to question (a) or (b) at one of the follow-up measurements; incident suicide attempt was defined as a positive answer to question (c) at follow-up. Individuals with either suicidal ideation or suicide attempts at baseline were excluded from the analyses.

\section{Symptom domains}

In each prospective cohort, a continuous symptom scale was constructed for depression, anxiety and mania symptoms at baseline by summing the subject's binary scores of the individual items of the respective CIDI sections, thus generating a continuous scale for each symptom domain (van Nierop et al. 2015a). The section on depression included 27 symptoms in EDSP, 19 symptoms in NEMESIS and 24 symptoms in NEMESIS-2. Items on suicidal ideation and suicide attempts were excluded from the continuous scale for depression symptoms. The section on mania included 11 symptoms in both EDSP and NEMESIS, and 17 symptoms in NEMESIS-2. Finally, the section on anxiety included 40 symptoms in EDSP, 54 symptoms in NEMESIS and 43 items in NEMESIS-2. Since the number of depressive, anxiety and mania symptoms varied among the CIDI-versions that were used in the different studies, the symptom scales were standardized in each 
cohort by dividing the sum scores by their standard deviations before merging the three databases. In addition, quadratic terms were generated for the various symptom scales, since the association between symptom domains and suicidal ideation and behaviour may be nonlinear (Schiepek et al. 2011).

\section{Statistical analyses}

Statistical analyses were performed in Stata version 13.0 (StataCorp. 2013). The three prospective datasets were first prepared by identifying and renaming variables and by generating identification variables. Then, databases were merged using the append command in Stata. As the research question focussed on association rather than incidence or prevalence, sampling weights were not applied. In order to adjust for clustering of data within studies, all regression models included two dummies for the study (study-ID) (Snijders and Bosker 2011). Only participants with complete data were analysed. Participants with psychotic disorder at baseline were excluded from all analyses. The outcome variables were incident suicidal ideation and incident suicide attempt.

First, the association between baseline PE and suicidal ideation or suicide attempts at follow-up was tested in a logistic regression model including baseline PE, study-ID, age and sex as the independent variables and suicidal ideation or suicide attempts as the dependent variable. To sensitively correct for the influence of depression, anxiety and mania symptoms, adjusted regression models included the continuous measures of these symptom domains as covariates. To assess whether the association between the various symptom domains and suicidal ideation and suicide attempts was linear, both the linear and the quadratic term of the continuous symptom variables were added to the regression model. If the quadratic term did not reach significance, only the linear term was used. If the quadratic term reached statistical significance, both the quadratic term and the linear term were used. In the final model, demographic variables and all three symptom scales were added simultaneously.

Second, all analyses were repeated in the following subgroups: (1) individuals without a diagnosis of any mental disorder (i.e. any anxiety disorder, major depressive episode, dysthymia, any substance use disorder, bipolar disorder or psychotic disorder) at baseline; (2) individuals with any baseline mental disorder (as defined for first group).

Third, to further assess the influence of baseline non-psychotic mental disorders on the association between PE and suicidal ideation and behaviour, and to examine doseresponse relationships between the co-occurrence of mental disorders and $\mathrm{PE}$, an additional logistic regression was performed with a categorical variable as main independent variable including the following categories: (1) No PE and no baseline mental disorder, (2) PE but no baseline mental disorder, (3) PE and one baseline mental disorder, (4) $\mathrm{PE}$ and two or more baseline mental disorders.

Finally, to assess whether the co-occurrence of various symptom domains next to PE increased the risk of suicidal ideation and behaviour in a dose-response fashion in par- 
ticipants without baseline mental disorders, a logistic regression model was used with a categorical predictor variable containing the following categories (van Nierop et al. 2015b; van Nierop et al. 2015a): (1) No PE and no anxiety, depression or mania symptoms, (2) PE but no anxiety, depression or mania symptoms, (3) PE and one symptom dimension (anxiety, depression or mania) present, (4) PE and two symptom dimensions present, (5) PE and three symptom dimensions present.

\section{Results}

\section{Baseline characteristics}

Of the included participants, $13.0 \%(n=2065)$ reported lifetime PE at baseline. The group with PE was significantly younger, and the proportion of males was lower than in the group without PE (Table 1). The lifetime prevalence of all baseline mental disorders, suicidal ideation and suicide attempts was significantly higher in participants with PE compared to participants without PE. Furthermore, the mean number of baseline depression, anxiety or mania symptoms was significantly higher in the PE group. 


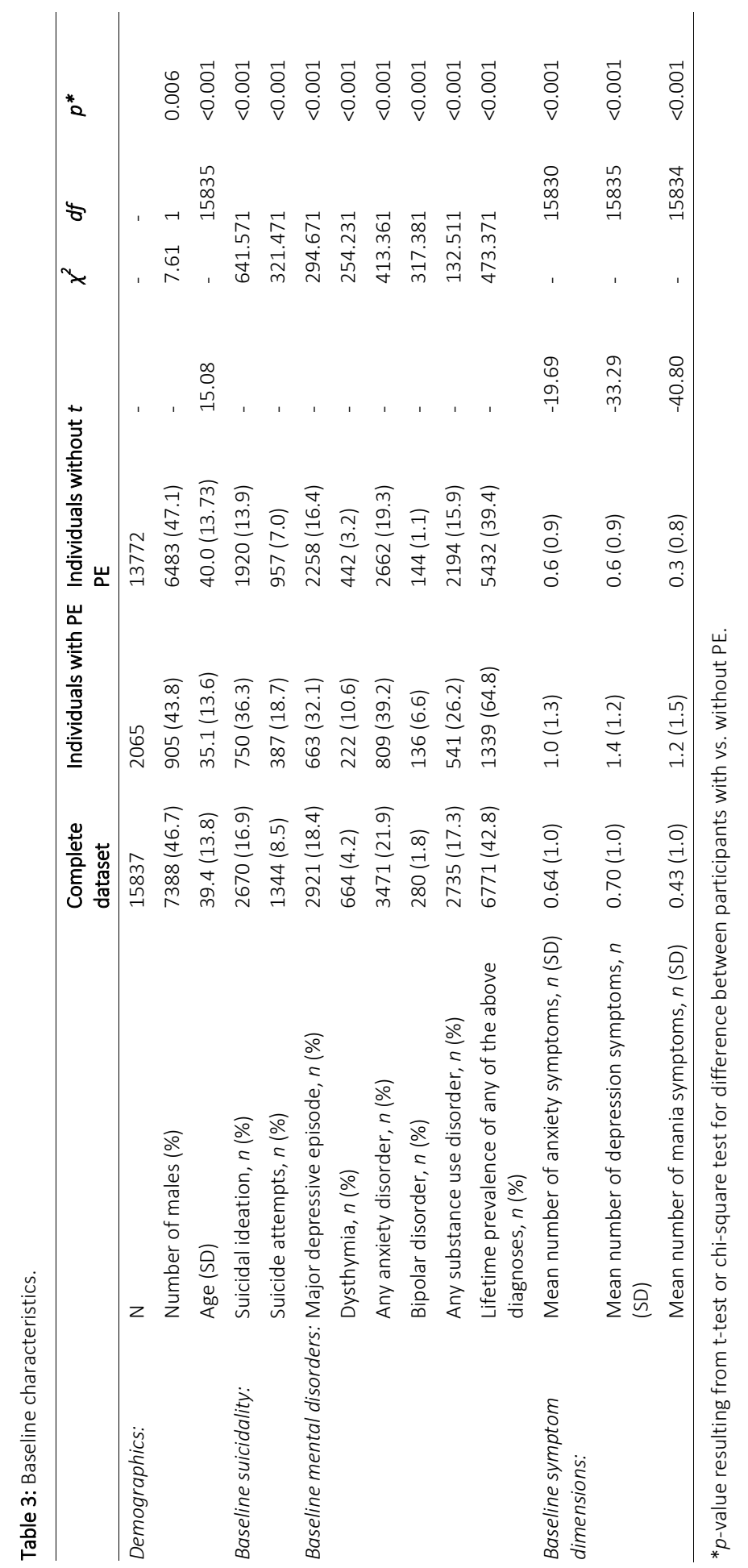


Associations between psychotic experiences and suicidal ideation and suicide attempts

\section{General population}

The OR of the association between PE and suicidal ideation was 2.07 (95\% Cl $1.50-$ 2.87; Table 2). Including continuous measures of depression, mania or anxiety symptoms in the model reduced the association considerably. Adding all three symptom scales simultaneously resulted in a $70 \%$ reduction of the excess risk associated with $\mathrm{PE}$ ( $O R=1.32 ; 95 \% \mathrm{Cl} 0.93-1.87$ ). The OR of the association between PE and suicide attempts was 3.95 (95\% Cl 1.85 - 8.43; Table 2). Adjustment for baseline depression, mania and anxiety symptoms slightly decreased the $O R$, but the association remained statistically significant (OR=3.44, 95\% Cl $1.54-7.69)$.

\section{Subgroup without baseline mental disorders}

In the subsample without a lifetime history of any mental disorder, the OR of the association between PE and suicidal ideation was 1.87 (95\% Cl 1.10 - 3.17; Table 2). Adjusting the analysis for depression, anxiety and mania symptoms resulted in a statistically non-significant association $(\mathrm{OR}=1.20,95 \% \mathrm{Cl} 0.68-2.10)$. There was no association between $\mathrm{PE}$ and suicide attempts in this subsample.

Subgroup with any baseline mental disorders

In the subsample with any baseline mental disorder, the OR of the association between PE and suicidal ideation was 1.79 (95\% Cl 1.18 - 2.71; Table 2). Including continuous measures of non-psychotic symptomatology decreased ORs, resulting in a nonsignificant OR of 1.37 ( $95 \% \mathrm{Cl} 0.89-2.13)$. The OR of the association between PE and suicide attempts was 8.90 ( $95 \% \mathrm{Cl} 2.91-27.21$; Table 2). Adjustment for baseline depression, mania and anxiety symptoms slightly increased the OR (OR=9.16, 95\% $\mathrm{Cl} 2.84$ $-29.52)$. 
Psychotic experiences and incident suicidal ideation and behaviour

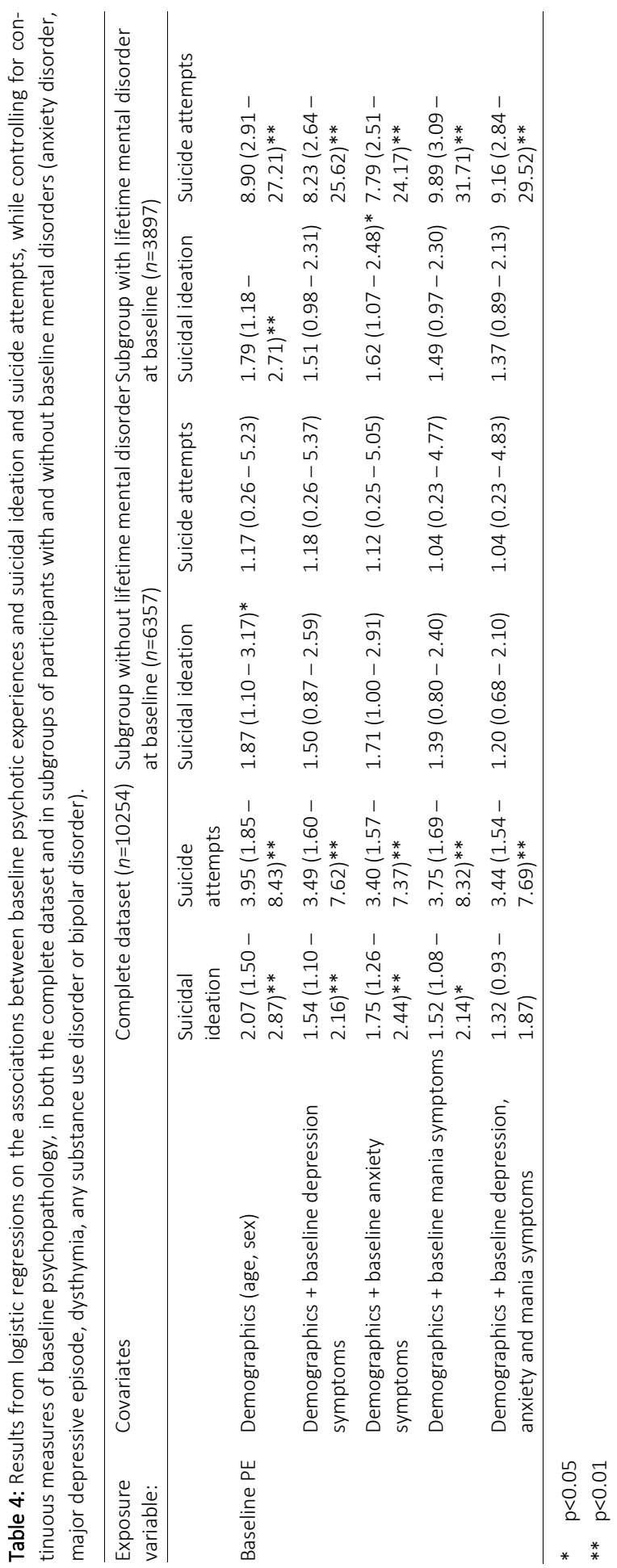




\section{Dose-response relationships with connected psychopathology}

\section{General population}

Participants with PE at baseline but without a lifetime comorbid mental disorder were at increased risk of suicidal ideation, compared to participants without PE and comorbid mental disorders (OR=1.95, 95\% Cl 1.15 - 3.29; Table 3). The risk of suicidal ideation increased in a dose-response fashion as the number of comorbid mental disorders at baseline increased, resulting in an OR of $2.73(95 \% \mathrm{Cl} 1.54-4.84)$ for participants with $\mathrm{PE}$ and one mental disorder and an OR of 5.50 (95\% Cl $3.20-9.45)$ for individuals with $\mathrm{PE}$ and two or more comorbid mental disorders. In the absence of a co-occurring mental disorder there was no association between PE and suicide attempts. However, PE were associated with suicide attempts when co-occurring with at least one mental disorder.

Table 3: Results from logistic regression in the complete dataset on the associations between psychotic experiences and suicidal ideation and suicide attempts in presence and absence of one or more mental disorders.

\begin{tabular}{lll}
\hline Baseline (complete sample, $n=6656)$ & Suicidal ideation, OR $(95 \% \mathrm{Cl})$ & Suicide attempt, OR $(95 \% \mathrm{Cl})$ \\
\hline No PE + no mental disorder & 1.00 (reference) & 1.00 (reference) \\
PE present + no mental disorder & $1.95(1.15-3.29)^{*}$ & $1.29(0.29-5.76)$ \\
PE present + 1 mental disorder & $2.73(1.54-4.84)^{* *}$ & $6.72(2.50-18.07)^{* *}$ \\
PE present + at least 2 mental disorders & $5.50(3.20-9.45)^{* *}$ & $5.20(1.46-18.50)^{*}$ \\
\hline
\end{tabular}

* $\quad \mathrm{p}<0.05$

** $\quad \mathrm{p}<0.01$

\section{Subgroup without baseline mental disorders}

The risk of suicidal ideation increased in participants with PE but without baseline mental disorders as the number of endorsed non-psychotic symptom domains increased (Table 4), ranging from 0.89 (95\% $\mathrm{Cl} 0.12-6.81)$ for participants with $\mathrm{PE}$ and no other symptom domain to $13.98(5.18$ - 37.71) for participants with PE and three other symptom domains. In the subgroup without baseline mental disorder there were only two cases of incident suicide attempts, thus making it impossible to validly examine doseresponse associations in this group. 
Table 4: Results from logistic regression on the associations between psychotic experiences and suicidal ideation and suicide attempts in presence or absence of one or more symptom domains, in a subgroup of patients without mental disorders at baseline.

\begin{tabular}{lll}
\hline $\begin{array}{l}\text { Baseline (subgroup without mental disorders, } \\
n=2918)\end{array}$ & Suicidal ideation, OR $(95 \% \mathrm{Cl})$ & Suicide attempt, OR $(05 \% \mathrm{Cl})$ \\
\hline No PE + 0 symptom domains & 1.00 (reference) & 1.00 (reference) \\
PE present + 0 symptom domains & $0.89(0.12-6.81)$ & N.A. \\
PE present + 1 symptom domains & $2.12(0.59-7.64)$ & N.A. \\
PE present + 2 symptom domains & $5.36(2.08-13.82)^{* *}$ & $1.58(0.18-13.74)$ \\
PE present + 3 symptom domains & $13.98(5.18-37.71)^{* *}$ & $3.57(0.40-31.48)$ \\
\hline
\end{tabular}

* $\quad \mathrm{p}<0.05$

$* * \quad p<0.01$

\section{Discussion}

\section{Main findings}

The present study is the first to analyse the longitudinal association between PE and suicidal ideation and behaviour merging multiple prospective, general population cohorts. In the complete sample, individuals with baseline PE were at increased risk of suicidal ideation and suicide attempts at follow-up. After adjustment for baseline anxiety, depression and mania symptoms, PE were no longer associated with suicidal ideation. However, PE remained significantly associated with suicide attempts. Further examination of these associations in subgroups of individuals with and without baseline mental disorders revealed that PE were only associated with suicide attempts in the subgroup with any baseline mental disorder. In the subgroup without baseline mental disorder, the risk of suicidal ideation increased as the number of co-occurring symptom domains increased.

\section{Interpretation}

A recent meta-analysis concluded that general population individuals with PE are at increased risk of suicidal ideation and behaviour, but that this association is confounded by the presence of co-occurring non-psychotic psychopathology (Honings et al. 2016). The reported association between PE and suicidal ideation and behaviour was hypothesized to reflect the increased risk of these outcomes in distressed participants with comorbid mental disorders or high levels of psychopathology. The present study supports this hypothesis.

After adjustment for depression, anxiety and mania symptoms, PE were no longer associated with suicidal ideation, both in the complete sample and in subgroups with and without baseline mental disorders, indicating that the association is confounded by 
non-psychotic psychopathology. Similarly, in the group without baseline mental disorders, the risk of suicidal ideation increased as the number of co-endorsed symptom domains increased. Previous research showed that the earliest expressions of psychopathology are nonspecific and contain a mixture of psychotic, affective, and anxiety symptoms that dynamically affect each other, and gradually differentiate into more specific syndromes over time, thus resulting in a 'general distress syndrome' (Wigman et al. 2013; McGorry and van Os 2013; van Os 2013). Therefore, the finding that the cooccurrence of PE with other symptom domains is associated with an increased risk of suicidal ideation, likely reflects the increased risk of suicidal ideation in participants who do not yet meet criteria for a diagnosis, but possibly display early expressions of psychopathology.

In the complete sample, individuals with PE were at increased risk of suicide attempts, even after adjustment for depression, anxiety and mania symptoms. However, examination of this association in subgroups stratified by presence or absence of baseline mental disorder revealed that PE were only associated with suicide attempts in presence of at least one mental disorder. This finding supports the hypothesis of the present study that the association between PE and suicide attempts reflects the increased risk of suicide attempts in participants with more severe mental distress in the context of mental disorders. Previous cross-sectional (DeVylder et al. 2015b) and longitudinal (Kelleher et al. 2013) studies reported similar results. In the subgroup without baseline mental disorders, there was no association between PE and suicide attempts.

The present study shows that PE are a risk factor for suicide attempts in individuals with mental disorders. However, the mechanism behind this association remains unclear. Previous work identified shared common causes for PE and suicide attempts, including psychological and social factors (DeVylder et al. 2015a), increased emotional reactivity to stress (Lataster et al. 2009), emotion oriented coping styles (Lin et al. 2011) and exposure to traumatic life events (Iga et al. 2007). However, more research on underlying mechanisms is required. The results of the present study show that PE serve as an indicator of illness severity and poor outcome in persons with non-psychotic psychopathology, in line with previous research (Wigman et al. 2012; Wigman et al. 2014; Perlis et al. 2011; Guloksuz et al. 2015; Kelleher et al. 2012b). Therefore, assessment of PE in individuals with non-psychotic psychopathology should be incorporated both in clinical practice and in future research, as previously suggested (Kelleher et al. 2013), in order to identify individuals at risk of suicidal behaviour. In addition, future research should examine the role of PE in the transition of suicidal ideation to suicide attempts, since evidence shows that most risk factors for suicidal ideation do not distinguish between ideators and attempters (Klonsky and May 2014; May and Klonsky 2016). 


\section{Strengths and limitations}

Strength of the present study is the large sample size, including both adults and adolescents. Moreover, a three year follow-up is longer than follow-up periods in most previous studies (Kelleher et al. 2013; Martin et al. 2015; Sullivan et al. 2015), thus increasing the likelihood of suicidal ideation or suicide attempts to occur, and enhancing statistical power. The present study is also the first longitudinal study that included dimensional symptom scales in the statistical analyses, and used general population samples stratified by the presence or absence of mental disorders in an attempt to disentangle longitudinal associations between PE and suicidal ideation and suicide attempts from connected psychopathology, while minimizing residual confounding.

The results of the present study should be interpreted in the light of some methodological limitations. First, the outcome of incident suicide attempt was rare. Even though three general population cohorts were merged into one database, the statistical power was low in some analyses, resulting in wide $95 \%$ confidence intervals.

Second, the individual studies that were merged into one dataset for this analysis differed with regard to study design and assessment of PE. NEMESIS and NEMESIS-2 had three year follow-up periods, while the EDSP study had a five year follow-up. In order to obtain sufficient power, the data sets were merged and analysed as if the follow-up periods were identical, based on the assumption that the three-year risk of suicidal ideation and suicide attempts is not substantially different from the five-year risk of suicidal behaviour (Nock et al. 2012). Moreover, the present study included both selfreported PE (EDSP data, albeit elicited by interviewers who were allowed to probe with clinical follow-up questions), clinically validated PE (NEMESIS-2 data) and partially clinically validated PE (NEMESIS). To examine whether these methodological differences between the individual studies influenced the results, sensitivity analyses were conducted in the individual datasets (results not shown). Although analyses had low statistical power and 95\% confidence intervals were wide, ORs in EDSP and NEMESIS data, separately, were similar. However, ORs in NEMESIS-2 were larger than in EDSP and NEMESIS. A possible explanation for this difference is that in NEMESIS-2 only clinically validated PE were used. Previous work showed that individuals with clinically validated PE have more non-psychotic psychopathology and seek help for their PE more often, compared with individuals with self-reported PE (van Nierop et al. 2012). This might explain why clinically validated PE are associated more strongly with suicidal ideation and suicide attempts than self-reported PE or partially clinically validated PE. Nevertheless, evidence suggests that self-reported PE are also predictive of several poor mental health outcomes and measure the same underlying construct as clinically validated PE (Bak et al. 2003; Kaymaz et al. 2012; Poulton et al. 2000; van Nierop et al. 2012). Therefore, both assessments of PE can be validly combined into one variable. Moreover, all regression models included two dummies for the studies to adjust for clustering of data within studies. 
Third, the mental disorders that were diagnosed in the three individual studies were

not fully congruent. To reach conformity among the studies, it was necessary to restrict the included mental disorders to the ones that were measured across all three studies. Inclusion of more mental disorders might have resulted in stronger evidence that PE are mostly predictive of suicide attempts in participants with co-occurring mental disorders.

Fourth, the three year follow-up period can be considered a limitation as well, since suicidality may fluctuate over a longer follow-up period (Gabennesch 1988; Noble 1996). Moreover, from a suicide prevention perspective, a shorter follow-up period could be useful, since it provides information on impending acts. However, previous longitudinal work assessed the association between PE and suicide attempts at three and twelve months after baseline and found similar results as the present study (Kelleher et al. 2013).

Fifth, follow-up data was missing for a substantial proportion of the participants (25\%). Attrition occurred more often in individuals with PE $(O R=1.23,95 \% \mathrm{Cl} 1.11$ 1.37). However, further examination of attrition in the individual datasets showed that PE were only associated with attrition in NEMESIS. Previous work showed that attrition in NEMESIS was higher in respondents with one or more mental disorders (de Graaf et al. 2000). Therefore, the association between PE and attrition found in the present study, reflects the increased risk of attrition in individuals with mental disorders in NEMESIS; in individuals without mental disorders PE were not associated with attrition. Since individuals with PE and mental disorders are also at risk of suicidal ideation and behaviour, the reported associations might have been stronger in absence of attrition.

Finally, the CIDI version used in NEMESIS-2 included a screening section with key questions for most mental disorders. Therefore, not all participants answered all questions about depression, anxiety or mania symptoms. This may have caused an underestimation of the symptom scores in this study.

In conclusion, the present study showed that PE in the general population are associated with an increased risk of suicidal ideation and behaviour. However, these associations are not specific, but reflect the increased risk of suicidal ideation in individuals with subthreshold multidimensional psychopathology and suicide attempts in individuals with co-occurring mental disorders.

\section{Acknowledgment}

Financial support for NEMESIS-1 and NEMESIS-2 was received from the Ministry of Health, Welfare and Sport, with supplement support from The Netherlands Organization for Health Research and Development (ZonMw), the Genetic Risk and Outcome of Psychosis (GROUP) Investigators, and the National Institute for Public Health and Environment (RIVM). This work is part of the EDSP Study and is funded by the German Federal Ministry of Education and Research (BMBF) project no. 01EB9405/6, 01EB9910/6, 
EB10106200, 01EB0140 and 01EB0440. Part of the fieldwork and analyses were also additionally supported by grants of the Deutsche Forschungsgemeinschaft (DFG) LA1148/1-1, WI2246/7-1 and WI709/8-1. Principal investigators of the EDSP Study are H.U. Wittchen and R. Lieb. Core staff members of the EDSP group are: K. von Sydow, G. Lachner, A. Perkonigg, P. Schuster, M. Höfler, H. Sonntag, T. Brückl, E. Garczynski, B. Isensee, A. Nocon,

C. Nelson, H. Pfister, V. Reed, B. Spiegel, A. Schreier, U. Wunderlich, P. Zimmermann, D. Beesdo and A. Bitner. Scientific advisors are J. Angst (Zurich), J. Margraf (Basel), G. Esser (Potsdam), K. Merikangas (National Institute of Mental Health, Bethesda), R. Kessler (Harvard, Boston) and J. van Os (Maastricht). 


\section{References}

Alonso, J., Angermeyer, M. C., Bernert, S., Bruffaerts, R., Brugha, T. S., Bryson, H., De Girolamo, G., Graaf, R., Demyttenaere, K., Gasquet, I., Haro, J. M., Katz, S. J., Kessler, R. C., Kovess, V., Lepine, J. P., Ormel, J., Polidori, G., Russo, L. J., Vilagut, G., Almansa, J., Arbabzadeh-Bouchez, S., Autonell, J., Bernal, M., BuistBouwman, M. A., Codony, M., Domingo-Salvany, A., Ferrer, M., Joo, S. S., Martinez-Alonso, M., Matschinger, H., Mazzi, F., Morgan, Z., Morosini, P., Palacin, C., Romera, B., Taub, N., Vollebergh, W. A. \& Esemed/Mhedea Investigators, E. S. O. T. E. O. M. D. P. 2004. Sampling and methods of the European Study of the Epidemiology of Mental Disorders (ESEMeD) project. Acta Psychiatr Scand Suppl, 8-20.

Bak, M., Delespaul, P., Hanssen, M., De Graaf, R., Vollebergh, W. \& Van Os, J. 2003. How false are "false" positive psychotic symptoms? Schizophr Res, 62, 187-9.

Bijl, R. V., Van Zessen, G., Ravelli, A., De Rijk, C. \& Langendoen, Y. 1998. The Netherlands Mental Health Survey and Incidence Study (NEMESIS): objectives and design. Soc Psychiatry Psychiatr Epidemiol, 33, 581-6.

Bostwick, J. M. \& Pankratz, V. S. 2000. Affective disorders and suicide risk: a reexamination. Am J Psychiatry, 157, 1925-32.

Calkins, M. E., Moore, T. M., Merikangas, K. R., Burstein, M., Satterthwaite, T. D., Bilker, W. B., Ruparel, K., Chiavacci, R., Wolf, D. H., Mentch, F., Qiu, H., Connolly, J. J., Sleiman, P. A., Hakonarson, H., Gur, R. C. \& Gur, R. E. 2014. The psychosis spectrum in a young U.S. community sample: findings from the Philadelphia Neurodevelopmental Cohort. World Psychiatry, 13, 296-305.

Capra, C., Kavanagh, D. J., Hides, L. \& Scott, J. G. 2015. Subtypes of psychotic-like experiences are differentially associated with suicidal ideation, plans and attempts in young adults. Psychiatry Res, 228, 894-8.

Cavanagh, J. T., Carson, A. J., Sharpe, M. \& Lawrie, S. M. 2003. Psychological autopsy studies of suicide: a systematic review. Psychol Med, 33, 395-405.

Cougnard, A., Marcelis, M., Myin-Germeys, I., De Graaf, R., Vollebergh, W., Krabbendam, L., Lieb, R., Wittchen, H. U., Henquet, C., Spauwen, J. \& Van Os, J. 2007. Does normal developmental expression of psychosis combine with environmental risk to cause persistence of psychosis? A psychosis proneness-persistence model. Psychol Med, 37, 513-27.

De Graaf, R., Bijl, R. V., Smit, F., Ravelli, A. \& Vollebergh, W. A. 2000. Psychiatric and sociodemographic predictors of attrition in a longitudinal study: The Netherlands Mental Health Survey and Incidence Study (NEMESIS). Am J Epidemiol, 152, 1039-47.

De Graaf, R., Ormel, J., Ten Have, M., Burger, H. \& Buist - Bouwman, M. 2008. Mental disorders and service use in the Netherlands: Results from the European Study of the Epidemiology of Mental Disorders (ESEMeD). The WHO World Mental Health Surveys: Global Perspectives on the Epidemiology of Mental Disorders. New York: Cambridge University Press.

De Graaf, R., Ten Have, M. \& Van Dorsselaer, S. 2010. The Netherlands Mental Health Survey and Incidence Study-2 (NEMESIS-2): design and methods. Int J Methods Psychiatr Res, 19, 125-41.

Devylder, J. E., Burnette, D. \& Yang, L. H. 2014. Co-occurrence of psychotic experiences and common mental health conditions across four racially and ethnically diverse population samples. Psychol Med, 44, 3503-13.

Devylder, J. E., Jahn, D. R., Doherty, T., Wilson, C. S., Wilcox, H. C., Schiffman, J. \& Hilimire, M. R. 2015a. Social and psychological contributions to the co-occurrence of sub-threshold psychotic experiences and suicidal behavior. Soc Psychiatry Psychiatr Epidemiol, 50, 1819-30.

Devylder, J. E., Lukens, E. P., Link, B. G. \& Lieberman, J. A. 2015b. Suicidal ideation and suicide attempts among adults with psychotic experiences: data from the Collaborative Psychiatric Epidemiology Surveys. JAMA Psychiatry, 72, 219-25.

Eaton, W. W., Neufeld, K., Chen, L. S. \& Cai, G. 2000. A comparison of self-report and clinical diagnostic interviews for depression: diagnostic interview schedule and schedules for clinical assessment in neuropsychiatry in the Baltimore epidemiologic catchment area follow-up. Arch Gen Psychiatry, 57, 217-22.

Fisher, H. L., Caspi, A., Poulton, R., Meier, M. H., Houts, R., Harrington, H., Arseneault, L. \& Moffitt, T. E. 2013. Specificity of childhood psychotic symptoms for predicting schizophrenia by 38 years of age: a birth cohort study. Psychol Med, 43, 2077-86. 
Gabennesch, H. 1988. When Promises Fail: A Theory of Temporal Fluctuations in Suicide*. Social Forces, 67, 129-145.

Gevonden, M. J., Selten, J. P., Myin-Germeys, I., De Graaf, R., Ten Have, M., Van Dorsselaer, S., Van Os, J. \& Veling, W. 2014. Sexual minority status and psychotic symptoms: findings from the Netherlands Mental Health Survey and Incidence Studies (NEMESIS). Psychol Med, 44, 421-33.

Guloksuz, S., Van Nierop, M., Lieb, R., Van Winkel, R., Wittchen, H. U. \& Van Os, J. 2015. Evidence that the presence of psychosis in non-psychotic disorder is environment-dependent and mediated by severity of non-psychotic psychopathology. Psychol Med, 45, 2389-401.

Haro, J. M., Arbabzadeh-Bouchez, S., Brugha, T. S., De Girolamo, G., Guyer, M. E., Jin, R., Lepine, J. P., Mazzi, F., Reneses, B., Vilagut, G., Sampson, N. A. \& Kessler, R. C. 2006. Concordance of the Composite International Diagnostic Interview Version 3.0 (CIDI 3.0) with standardized clinical assessments in the WHO World Mental Health surveys. Int J Methods Psychiatr Res, 15, 167-80.

Hawton, K. \& Van Heeringen, K. 2009. Suicide. Lancet, 373, 1372-81.

Honings, S., Drukker, M., Groen, R. \& Van Os, J. 2016. Psychotic experiences and risk of self-injurious behaviour in the general population: a systematic review and meta-analysis. Psychol Med, 46, 237-51.

Iga, J., Ueno, S., Yamauchi, K., Numata, S., Tayoshi-Shibuya, S., Kinouchi, S., Nakataki, M., Song, H., Hokoishi, K., Tanabe, H., Sano, A. \& Ohmori, T. 2007. The Val66Met polymorphism of the brain-derived neurotrophic factor gene is associated with psychotic feature and suicidal behavior in Japanese major depressive patients. Am J Med Genet B Neuropsychiatr Genet, 144B, 1003-6.

Jahn, D. R., Devylder, J. E. \& Hilimire, M. R. 2016. Explanatory risk factors in the relations between schizotypy and indicators of suicide risk. Psychiatry Res, 238, 68-73.

Jang, J. H., Lee, Y. J., Cho, S. J., Cho, I. H., Shin, N. Y. \& Kim, S. J. 2014. Psychotic-like experiences and their relationship to suicidal ideation in adolescents. Psychiatry Res, 215, 641-5.

Jeppesen, P., Clemmensen, L., Munkholm, A., Rimvall, M. K., Rask, C. U., Jorgensen, T., Larsen, J. T., Petersen, L., Van Os, J. \& Skovgaard, A. M. 2015. Psychotic experiences co-occur with sleep problems, negative affect and mental disorders in preadolescence. J Child Psychol Psychiatry, 56, 558-65.

Kaymaz, N., Drukker, M., Lieb, R., Wittchen, H. U., Werbeloff, N., Weiser, M., Lataster, T. \& Van Os, J. 2012. Do subthreshold psychotic experiences predict clinical outcomes in unselected non-help-seeking populationbased samples? A systematic review and meta-analysis, enriched with new results. Psychol Med, 42, 2239-53.

Kelleher, I., Connor, D., Clarke, M. C., Devlin, N., Harley, M. \& Cannon, M. 2012a. Prevalence of psychotic symptoms in childhood and adolescence: a systematic review and meta-analysis of population-based studies. Psychol Med, 42, 1857-63.

Kelleher, I., Keeley, H., Corcoran, P., Lynch, F., Fitzpatrick, C., Devlin, N., Molloy, C., Roddy, S., Clarke, M. C., Harley, M., Arseneault, L., Wasserman, C., Carli, V., Sarchiapone, M., Hoven, C., Wasserman, D. \& Cannon, M. 2012b. Clinicopathological significance of psychotic experiences in non-psychotic young people: evidence from four population-based studies. Br J Psychiatry, 201, 26-32.

Kelleher, I., Lynch, F., Harley, M., Molloy, C., Roddy, S., Fitzpatrick, C. \& Cannon, M. 2012c. Psychotic symptoms in adolescence index risk for suicidal behavior: findings from 2 population-based case-control clinical interview studies. Arch Gen Psychiatry, 69, 1277-83.

Kelleher, I., Corcoran, P., Keeley, H., Wigman, J. T., Devlin, N., Ramsay, H., Wasserman, C., Carli, V., Sarchiapone, M., Hoven, C., Wasserman, D. \& Cannon, M. 2013. Psychotic symptoms and population risk for suicide attempt: a prospective cohort study. JAMA Psychiatry, 70, 940-8.

Klonsky, E. D. \& May, A. M. 2014. Differentiating suicide attempters from suicide ideators: a critical frontier for suicidology research. Suicide Life Threat Behav, 44, 1-5.

Koyanagi, A., Stickley, A. \& Haro, J. M. 2015. Subclinical psychosis and suicidal behavior in England: Findings from the 2007 Adult Psychiatric Morbidity Survey. Schizophr Res, 168, 62-7.

Lataster, T., Wichers, M., Jacobs, N., Mengelers, R., Derom, C., Thiery, E., Van Os, J. \& Myin-Germeys, I. 2009. Does reactivity to stress cosegregate with subclinical psychosis? A general population twin study. Acta Psychiatr Scand, 119, 45-53. 
Lewis-Fernandez, R., Horvitz-Lennon, M., Blanco, C., Guarnaccia, P. J., Cao, Z. \& Alegria, M. 2009. Significance of endorsement of psychotic symptoms by US Latinos. J Nerv Ment Dis, 197, 337-47.

Lieb, R., Isensee, B., Von Sydow, K. \& Wittchen, H. U. 2000. The Early Developmental Stages of Psychopathology Study (EDSP): a methodological update. Eur Addict Res, 6, 170-82.

Lin, A., Wigman, J. T., Nelson, B., Vollebergh, W. A., Van Os, J., Baksheev, G., Ryan, J., Raaijmakers, Q. A., Thompson, A. \& Yung, A. R. 2011. The relationship between coping and subclinical psychotic experiences in adolescents from the general population--a longitudinal study. Psychol Med, 41, 2535-46.

Linscott, R. J. \& Van Os, J. 2013. An updated and conservative systematic review and meta-analysis of epidemiological evidence on psychotic experiences in children and adults: on the pathway from proneness to persistence to dimensional expression across mental disorders. Psychol Med, 43, 1133-49.

Martin, G., Thomas, H., Andrews, T., Hasking, P. \& Scott, J. G. 2015. Psychotic experiences and psychological distress predict contemporaneous and future non-suicidal self-injury and suicide attempts in a sample of Australian school-based adolescents. Psychol Med, 45, 429-37.

May, A. M. \& Klonsky, E. D. 2016. What Distinguishes Suicide Attempters From Suicide Ideators? A MetaAnalysis of Potential Factors. Clinical Psychology: Science and Practice, 23, 5-20.

Mcgorry, P. \& Van Os, J. 2013. Redeeming diagnosis in psychiatry: timing versus specificity. Lancet, 381, 343-5. Miller, G. A. \& Chapman, J. P. 2001. Misunderstanding analysis of covariance. J Abnorm Psychol, 110, 40-8.

Murphy, J., Shevlin, M., Houston, J. \& Adamson, G. 2012. A population based analysis of subclinical psychosis and help-seeking behavior. Schizophr Bull, 38, 360-7.

Nishida, A., Sasaki, T., Nishimura, Y., Tanii, H., Hara, N., Inoue, K., Yamada, T., Takami, T., Shimodera, S., Itokawa, M., Asukai, N. \& Okazaki, Y. 2010. Psychotic-like experiences are associated with suicidal feelings and deliberate self-harm behaviors in adolescents aged 12-15 years. Acta Psychiatr Scand, 121, 301-7.

Noble, R. E. S. 1996. Temporal Fluctuations in Suicide Calls to a Crisis Intervention Service. Suicide and LifeThreatening Behavior, 26, 415-423.

Nock, M., Borges, G. \& Ono, Y. 2012. Suicide: Global Perspectives from the WHO World Mental Health Surveys, Cambridge University Press.

Nock, M. K., Borges, G., Bromet, E. J., Cha, C. B., Kessler, R. C. \& Lee, S. 2008. Suicide and suicidal behavior. Epidemiol Rev, 30, 133-54.

Nock, M. K. 2010. Self-injury. Annu Rev Clin Psychol, 6, 339-63.

Nordentoft, M., Mortensen, P. B. \& Pedersen, C. B. 2011. Absolute risk of suicide after first hospital contact in mental disorder. Arch Gen Psychiatry, 68, 1058-64.

O'connor, R. C., Platt, S. \& Gordon, J. 2011. Achievements and Challenges in Suicidology: Conclusions and Future Directions. International Handbook of Suicide Prevention. John Wiley \& Sons, Ltd.

Olfson, M., Lewis-Fernandez, R., Weissman, M. M., Feder, A., Gameroff, M. J., Pilowsky, D. \& Fuentes, M. 2002. Psychotic symptoms in an urban general medicine practice. Am J Psychiatry, 159, 1412-9.

Palmer, B. A., Pankratz, V. S. \& Bostwick, J. M. 2005. The lifetime risk of suicide in schizophrenia: a reexamination. Arch Gen Psychiatry, 62, 247-53.

Perlis, R. H., Uher, R., Ostacher, M., Goldberg, J. F., Trivedi, M. H., Rush, A. J. \& Fava, M. 2011. Association between bipolar spectrum features and treatment outcomes in outpatients with major depressive disorder. Arch Gen Psychiatry, 68, 351-60.

Poulton, R., Caspi, A., Moffitt, T. E., Cannon, M., Murray, R. \& Harrington, H. 2000. Children's self-reported psychotic symptoms and adult schizophreniform disorder: a 15-year longitudinal study. Arch Gen Psychiatry, 57, 1053-8.

Reed, V., Gander, F., Pfister, H., Steiger, A., Sonntag, H., Trenkwalder, C., Sonntag, A., Hundt, W. \& Wittchen, H.-U. 1998. To what degree does the Composite International Diagnostic Interview (CIDI) correctly identify DSM-IV disorders? Testing validity issues in a clinical sample. International Journal of Methods in Psychiatric Research, 7, 142-155.

Saha, S., Scott, J. G., Johnston, A. K., Slade, T. N., Varghese, D., Carter, G. L. \& Mcgrath, J. J. 2011. The association between delusional-like experiences and suicidal thoughts and behaviour. Schizophr Res, 132, 197202. 
Schiepek, G., Fartacek, C., Sturm, J., Kralovec, K., Fartacek, R. \& Ploderl, M. 2011. Nonlinear dynamics: theoretical perspectives and application to suicidology. Suicide Life Threat Behav, 41, 661-75.

Sharifi, V., Eaton, W. W., Wu, L. T., Roth, K. B., Burchett, B. M. \& Mojtabai, R. 2015. Psychotic experiences and risk of death in the general population: 24-27 year follow-up of the Epidemiologic Catchment Area study. Br J Psychiatry, 207, 30-6.

Smeets, F., Lataster, T., Van Winkel, R., De Graaf, R., Ten Have, M. \& Van Os, J. 2013. Testing the hypothesis that psychotic illness begins when subthreshold hallucinations combine with delusional ideation. Acta Psychiatr Scand, 127, 34-47.

Smeets, R. M. W. \& Dingemans, P. M. a. J. 1993. Composite International Diagnostic Interview (CIDI) Versie 1.1, Amerstam/Geneva, World Health Organisation.

Snijders, T. a. B. \& Bosker, R. J. 2011. Multilevel Analysis: An Introduction to Basic and Advanced Multilevel Modeling, SAGE Publications.

Statacorp. 2013. Stata Statistical Software: Release 13. College Station, TX: StataCorp LP.

Sullivan, S. A., Lewis, G., Gunnell, D., Cannon, M., Mars, B. \& Zammit, S. 2015. The longitudinal association between psychotic experiences, depression and suicidal behaviour in a population sample of adolescents. Soc Psychiatry Psychiatr Epidemiol, 50, 1809-17.

Sveticic, J. \& De Leo, D. 2012. The hypothesis of a continuum in suicidality: a discussion on its validity and practical implications. Ment IIIn, 4, e15.

Taylor, P. J., Hutton, P. \& Wood, L. 2015. Are people at risk of psychosis also at risk of suicide and self-harm? A systematic review and meta-analysis. Psychol Med, 45, 911-26.

Temmingh, H., Stein, D. J., Seedat, S. \& Williams, D. R. 2011. The prevalence and correlates of hallucinations in a general population sample: findings from the South African Stress and Health Study. Afr J Psychiatry (Johannesbg), 14, 211-7.

Ten Have, M., De Graaf, R., Van Dorsselaer, S., Verdurmen, J., Van 'T Land, H., Vollebergh, W. \& Beekman, A. 2009. Incidence and course of suicidal ideation and suicide attempts in the general population. Can J Psychiatry, 54, 824-33.

Ten Have, M., Van Dorsselaer, S. \& De Graaf, R. 2013. Prevalence and risk factors for first onset of suicidal behaviors in the Netherlands Mental Health Survey and Incidence Study-2. J Affect Disord, 147, 205-11.

Van Nierop, M., Van Os, J., Gunther, N., Myin-Germeys, I., De Graaf, R., Ten Have, M., Van Dorsselaer, S., Bak, M. \& Van Winkel, R. 2012. Phenotypically continuous with clinical psychosis, discontinuous in need for care: evidence for an extended psychosis phenotype. Schizophr Bull, 38, 231-8.

Van Nierop, M., Bak, M., De Graaf, R., Ten Have, M., Van Dorsselaer, S., Genetic, R., Outcome of Psychosis, I., Van Winkel, R., Genetic, R. \& Outcome of Psychosis, G. I. 2015a. The functional and clinical relevance of childhood trauma-related admixture of affective, anxious and psychosis symptoms. Acta Psychiatr Scand.

Van Nierop, M., Viechtbauer, W., Gunther, N., Van Zelst, C., De Graaf, R., Ten Have, M., Van Dorsselaer, S., Bak, M., Genetic, R., Investigators, O. U. O. P. \& Van Winkel, R. 2015b. Childhood trauma is associated with a specific admixture of affective, anxiety, and psychosis symptoms cutting across traditional diagnostic boundaries. Psychol Med, 45, 1277-88.

Van Os, J., Hanssen, M., Bijl, R. \& Ravelli, A. 2000. Straus (1969) revisited: A psychosis continuum in the general population? Schizophrenia Research, 45, 11-20.

Van Os, J., Hanssen, M., Bijl, R. V. \& Vollebergh, W. 2001. Prevalence of psychotic disorder and community level of psychotic symptoms: an urban-rural comparison. Arch Gen Psychiatry, 58, 663-8.

Van Os, J. 2013. The dynamics of subthreshold psychopathology: implications for diagnosis and treatment. Am J Psychiatry, 170, 695-8.

Van Rossum, I., Dominguez, M. D., Lieb, R., Wittchen, H. U. \& Van Os, J. 2011. Affective dysregulation and reality distortion: a 10-year prospective study of their association and clinical relevance. Schizophr Bull, 37, 561-71.

Wigman, J. T., Van Nierop, M., Vollebergh, W. A., Lieb, R., Beesdo-Baum, K., Wittchen, H. U. \& Van Os, J. 2012. Evidence that psychotic symptoms are prevalent in disorders of anxiety and depression, impacting on illness onset, risk, and severity--implications for diagnosis and ultra-high risk research. Schizophr Bull, 38, 247-57. 


\section{Chapter 3}

Wigman, J. T., Van Os, J., Thiery, E., Derom, C., Collip, D., Jacobs, N. \& Wichers, M. 2013. Psychiatric diagnosis revisited: towards a system of staging and profiling combining nomothetic and idiographic parameters of momentary mental states. PLoS One, 8, e59559.

Wigman, J. T., Van Os, J., Abidi, L., Huibers, M. J., Roelofs, J., Arntz, A., Kelleher, I. \& Peeters, F. P. 2014. Subclinical psychotic experiences and bipolar spectrum features in depression: association with outcome of psychotherapy. Psychol Med, 44, 325-36.

Wittchen, H. U. 1994. Reliability and validity studies of the WHO--Composite International Diagnostic Interview (CIDI): a critical review. J Psychiatr Res, 28, 57-84.

Wittchen, H. U. \& Pfister, H. 1997. DIA-X-Interviews: Manual für Screening-Verfahren und Interview; Interviewheft., Frankfurt, Swets \& Zeitlinger.

Wittchen, H. U., Perkonigg, A., Lachner, G. \& Nelson, C. B. 1998. Early developmental stages of psychopathology study (EDSP): objectives and design. Eur Addict Res, 4, 18-27.

World Health Organization 1990. Composite International Diagnostic Interview (CIDI), Geneva, World Health Organization.

World Health Organization 2014. Preventing suicide: A global imperative, Geneva, World Health Organization. 


\section{Chapter}

\section{Psychotic experiences and risk of violence perpetration and arrest in the general population: a prospective study}

Steven Honings ${ }^{1}$, Marjan Drukker ${ }^{1}$, Margreet ten Have ${ }^{2}$, Ron de Graaf ${ }^{2}$, Saskia van Dorsselaer ${ }^{2}, \mathrm{Jim}$ van $\mathrm{Os}^{1,3,4}$

1. Department of Psychiatry and Psychology, South Limburg Mental Health Research and Teaching Network, Maastricht University Medical Centre, Maastricht, the Netherlands.

2. Netherlands Institute of Mental Health and Addiction, Utrecht, the Netherlands

3. King's College London, King's Health Partners, Department of Psychosis Studies, Institute of Psychiatry, London, UK

4. Department of Psychiatry, Brain Center Rudolf Magnus Institute, University Medical Center Utrecht, Utrecht, The Netherlands.

Published as:

Honings, S., Drukker, M., Ten Have, M., De Graaf, R., Van Dorsselaer, S. \& Van Os, J. 2016. Psychotic Experiences and Risk of Violence Perpetration and Arrest in the General Population: A Prospective Study. PLoS One, 11, e0159023. 


\begin{abstract}
Background: In cross-sectional, general population studies, psychotic experiences have been associated with an increased risk of physical violence perpetration and arrest. However, longitudinal research on this topic is lacking. Moreover, it remains unclear whether subjects with psychotic experiences are also at risk of displaying psychological violence. The present study aims to investigate these associations.

Method: The longitudinal association between baseline psychotic experiences and sixyear incidence of violence perpetration and three-year incidence of arrest was studied in a prospective cohort of 6646 general population adults. Logistic regression analyses with varying levels of adjustment were performed in the complete sample and in subsamples stratified by presence or absence of baseline mental disorders.

Results: The presence of psychotic experiences at baseline increased the risk of physical violence, psychological violence and arrest at follow-up. However, adjustment for dimensional measures of psychopathology and contextual confounders reduced all associations considerably. After adjustment, both clinically validated (OR=3.59, 95\% Cl 1.09 - 11.81) and self-reported hallucinations (OR=2.83, 95\% $\mathrm{Cl} 1.057 .65)$ remained significantly associated with physical violence perpetration. Self-reported (OR=3.06, 95\% Cl $1.55-6.03$ ) and clinically validated delusions ( $\mathrm{OR}=3.24,95 \% \mathrm{Cl} 1.47-7.13$ ) were associated with an increased risk of arrest. There was no significant association between psychotic experiences and incident psychological violence in the fully adjusted model.

Conclusion: Specific psychotic experiences may differentially predict physical violence perpetration and arrest, even after adjustment for demographics, dimensional measures of psychopathology and contextual confounders. However, more longitudinal research with larger sample sizes is required to confirm these findings.
\end{abstract}




\section{Introduction}

Psychotic experiences (PE) are hallucinatory or delusional experiences falling below the threshold of a diagnosable psychotic disorder (Linscott and van Os 2013; Kelleher et al. 2012a). In the general population, PE have been associated with an increased risk of violence perpetration (Nederlof et al. 2012; Mojtabai 2006; Kinoshita et al. 2011; Swanson et al. 1996; Coid et al. 2016) and arrest (Mojtabai 2006). However, the temporal sequence remains unclear since longitudinal studies on this topic are lacking. Moreover, it remains unclear whether individuals with PE also have an increased risk of displaying psychological violence.

For many years, researchers have studied the association between mental disorders and the risk of violent behaviour (Monahan 1992; Link and Stueve 1995; Joyal et al. 2007). Studies show that individuals with various major mental disorders including psychotic disorders are at increased risk of violence perpetration (Douglas et al. 2009; Fazel et al. 2009; Large and Nielssen 2011; Fazel et al. 2010; Dowden and Brown 2002; Yu et al. 2012; Fazel et al. 2015). This link has been shown in both offender populations (Fazel and Danesh 2002) and general population samples (Swanson 1994; ten Have et al. 2014). However, the association between mental disorders and violence is likely to be confounded by several factors, including comorbid substance use (van Dorn et al. 2012) and contextual confounders like negative life events and low social support (ten Have et al. 2014). Moreover, the proportion of community violence related to mental disorders varies between $3 \%$ and $20 \%$, indicating that the vast majority of community violence cannot be attributed to mental disorders (Swanson 1994; Hodgins and Janson 2002; Brennan et al. 2000).

In contrast to the literature on violence and full-blown psychosis (Douglas et al. 2009; Fazel et al. 2009; Large and Nielssen 2011), studies on the association between low grade PE and violence are scarce. Cross-sectional studies have shown that PE in the general population are associated with an increased risk of interpersonal violence (Kinoshita et al. 2011; Mojtabai 2006; Swanson et al. 1996; Nederlof et al. 2012; Coid et al. 2016), violence towards objects (Kinoshita et al. 2011) and arrest (Mojtabai 2006). Moreover, studies in detained youth showed that PE were more prevalent among incarcerated adolescents than among adolescents in the general population (Vreugdenhil et al. 2004). Similarly, violent incarcerated boys showed significantly more psychotic symptomatology than less violent incarcerated boys (Lewis et al. 1979).

Previous studies on the association between PE and violent behaviour, while instructive, had some methodological limitations. First, all studies used cross-sectional study designs to investigate the association (Nederlof et al. 2012; Mojtabai 2006; Kinoshita et al. 2011; Swanson et al. 1996; Coid et al. 2016), thus making it impossible to infer the temporal sequence, which is required to verify the hypothesis that PE induce violence perpetration, rather than vice versa. Second, no studies adjusted the analyses for dimensional measures of psychopathology. Instead, adjustments were made for various dichotomously defined risk factors associated with violent behaviour, including sub- 
stance use and other mental disorders, thus leaving room for residual confounding (Nederlof et al. 2012; Mojtabai 2006; Kinoshita et al. 2011; Swanson et al. 1996; Coid et al. 2016). Third, studies used self-reported PE as their independent variable rather than clinically validated PE (Nederlof et al. 2012; Mojtabai 2006; Kinoshita et al. 2011; Swanson et al. 1996; Coid et al. 2016). Although studies have shown that self-report questionnaires can detect PE with high accuracy (Kelleher et al. 2011), clinical interviews with sufficient PE items provide the most reliable estimate (Linscott and van Os 2013). Finally, no previous study investigated the associations between PE and forms of violent behaviour other than physical violence, for example psychological violence, which has been similarly linked to various mental disorders (ten Have et al. 2014).

The present study aimed to examine the longitudinal association between both selfreported and clinically validated PE on the one hand, and incident physical violence perpetration, psychological violence perpetration and arrest on the other, using data from a prospective, general population cohort. Moreover, it assessed to what extent the association may be confounded by non-psychotic psychopathology, both at the level of dimensional symptom scores and at the level of mental disorders as defined in the fourth edition of the Diagnostic and Statistical Manual of Mental Disorders (DSMIV). Confounding by categorical mental disorder was examined using the method of stratification by presence or absence of baseline mental disorders. The influence of other demographic and contextual confounders identified in the literature was also examined. Finally, the present study analysed to what degree self-reported PE were similarly predictive of violent behaviour as clinically validated PE.

It was hypothesized that (i) PE would be longitudinally associated with an increased risk of incident physical violence, psychological violence and arrest; (ii) this association would be confounded by co-occurring non-psychotic psychopathology that is highly prevalent among subjects with PE (Kelleher et al. 2012b; van Os et al. 2000; DeVylder et al. 2014); (iii) a degree of association between PE and violence and arrest would remain even after taking into account confounding (ten Have et al. 2014); (iv) self-reported PE would be equally predictive of violence and arrest as clinically validated PE.

\section{Methods}

\section{Sample}

This study uses data of the second Netherlands Mental Health Survey and Incidence Study (NEMESIS-2), a longitudinal study of the prevalence, incidence, course and consequences of mental disorders in the Dutch general population (de Graaf et al. 2010). NEMESIS-2 was approved by the Medical Ethics Review Committee for Institutions on Mental Health Care (METIGG). Participants provided written informed consent to participate in the interview, after full written and verbal information about the study was given before and at the start of 
the baseline assessment. Participants were selected based on a multistage random sampling procedure. In the first wave (T0), a total of 6646 persons aged 18-64 years were included. Participants were approached for two follow-up surveys, respectively three years (T1) and six years (T2) after baseline. At T1, 5503 persons were interviewed again (response rate 83\%). At T2, 4618 persons were interviewed (response rate 69\%). The mean period between the baseline interview and second follow-up interview was 6 years and 6 days. A more comprehensive description of the design can be found elsewhere (de Graaf et al. 2010).

\section{Diagnostic instrument}

Participants were interviewed using the Composite International Diagnostic Interview Version 3.0 (CIDI 3.0), a fully structured lay-administered diagnostic interview generating DSMIV diagnoses (Kessler and Ustun 2004). An important difference between the CIDI 3.0 and earlier versions is that the CIDI 3.0 contains a screening section consisting of key questions for most mental disorders. Only when a participant answers positively to a key question of the screening section, the complete CIDI section for that mental disorder is administered. The disorders considered in this study included mood disorders (major depression, dysthymia, bipolar disorder), anxiety disorders (panic disorder, agora-phobia without panic disorder, social phobia, specific phobia, generalized anxiety disorder), substance use disorders (alcohol/drug abuse and dependence) and impulse-control disorders (attention deficit hyperactivity disorder, conduct disorder, oppositional defiant disorder). Furthermore, antisocial personality disorder was measured using questions from the International Personality Disorder Examination (Loranger et al. 1994), which are part of CIDI 3.0 (Lenzenweger et al. 2007; Huang et al. 2009). Impulse-control disorders were only assessed in subjects aged 18-44 years because of the possibility of recall bias in older participants (Kessler et al. 2007). All other mental disorders were assessed in all participants. Clinical calibration studies in various countries reported that the CIDI 3.0 assesses mood disorders (area under ROC curve (AUC) ranging between $0.75-0.93$ ), anxiety disorders (AUC=0.73) and substance use disorders (AUC 0.62 - 0.88) with good validity when clinical reappraisal interviews are used as the gold standard (Haro et al. 2006). In addition, a clinical reappraisal interview carried out in a subsample of the National Comorbidity Survey Replication performed in the USA also found a valid assessment of ADHD in the CIDI 3.0 (Fayyad et al. 2007). For the present analyses, lifetime diagnoses at baseline were used.

\section{Psychotic experiences at baseline}

Baseline psychotic experiences were assessed using a psychosis add-on instrument based on the section of psychotic symptoms in CIDI 1.1. The instrument consisted of 20 questions regarding lifetime psychotic experiences. The 20 items consisted of 15 delusional experiences and 5 hallucinatory experiences, described in detail elsewhere (Wigman et al. 2012). Individuals who reported at least one lifetime psychotic experience were contacted for clini- 
cal re-interview over the telephone by an experienced clinician at the level of psychologist or psychiatrist within 8 weeks after the initial interview. Re-interview took place with questions from the Structured Clinical Interview for DSM-IV, which assesses psychotic disorders with good reliability (Williams et al. 1992; Skre et al. 1991) . Findings from all re-interviews were discussed with a second clinician. Diagnoses of psychotic disorder were based on the data from the clinical re-interviews. Clinically validated PE were considered present when the psychotic nature of the self-reported psychotic experiences was confirmed at the clinical reinterview. Of all participants eligible for re-interview, 74\% was actually re-interviewed. There were no large or significant differences between individuals who participated in the clinical re-interview and those who did not with regard to demographics, baseline mental disorders (van Nierop et al. 2012), violence perpetration or arrest. For the present study, both selfreported PE and clinically validated PE were used. Self-reported or clinically validated PE were considered present if individuals reported any self-reported or clinically validated CIDI psychotic symptom, respectively. In addition, continuous scales of both self-reported and clinically validated PE were computed. Individuals with psychotic disorder at baseline were excluded in order to ensure analyses reflected effects of subclinical PE.

\section{Violence perpetration and arrest at baseline and follow-up}

Perpetration of both physical violence and psychological violence was assessed at baseline by asking participants about lifetime physical or psychological violence towards one of their current or former intimate partners or towards any of their children during upbringing. To increase the likelihood of these acts being reported, they were not mentioned as such but were listed in a booklet (lists A and B) and referred to by number. The items on list A referred to psychological violence and included name-calling, offending, belittling, punishing unjustly, blackmailing and threatening. The items on list B concerned physical violence and included kicking, biting, hitting, trying to wound with an object (gun, knife, piece of wood, pair of scissors, other object) or hot water. An additional question from the CIDI screener section was used to assess baseline physical violence by asking: "Have you ever had attacks of anger when all of a sudden you lost control and hit or tried to hurt someone?" At both follow-up interviews (T1, T2), participants were asked about physical and psychological violence between waves by using the same lists as at baseline. In contrast to baseline, violence at follow-up included acts against any person in general. To reduce the effects of this discrepancy, participants without a partner or children at baseline were excluded when analysing physical or psychological violence. Consistent with previous studies, psychological violence was defined as present if it occurred on two or more occasions and physical violence on one or more occasions (ten Have et al. 2014; Kessler et al. 2001; Miller et al. 2011). Incident psychological violence perpetration (hereafter: psychological violence) and incident physical violence perpetration (hereafter: physical violence) were defined as present if the participant displayed the respective violent behaviour at any of the follow-up surveys, while subjects with the respective violent behaviour at baseline were excluded. 
Self-reported arrest was obtained at baseline (T0) and at the first follow-up survey (T1) by asking participants if they had been arrested. Incident arrest was defined as arrest at follow-up, while subjects with lifetime arrest at baseline were excluded from the analyses.

\section{Baseline dimensional symptom scales}

Symptoms of all baseline mental disorders were assessed at baseline using the respective CIDI sections. For each mental disorder, including substance use disorders (32 items), bipolar disorder (17 items), major depression/dysthymia (28 items), anxiety disorders (43 items), attention deficit hyperactivity disorder (9 items), oppositional defiant disorder (11 items) and conduct disorder (11 items), dimensional symptom scales were constructed by summing the participant's binary scores of the individual items of the respective CIDI sections. In addition, a dimensional scale was constructed for antisocial personality by summing the individual items regarding antisocial personality using questions from the International Personality Disorder Examination (7 items). Thus, eight continuous symptom scales were generated.

\section{Childhood trauma}

Childhood trauma was assessed at baseline by asking participants about physical, psychological or sexual abuse before the age of 16 years. Conform previous analyses (ten Have et al. 2014; de Graaf et al. 2004), childhood trauma was defined present if a participant had experienced psychological abuse on two or more occasions, or physical abuse / sexual abuse on one or more occasion.

\section{Social support}

Social support from three resources (partner, family or friends, neighbours) was assessed at T1 with two questions on instrumental and emotional support from each of these resources in the direct network. These referred to the extent participants could rely on them for help if they had a problem and could open up to them if they needed to talk about worries. The four response categories ranged from 'not at all' to 'a lot'. The mean score on both questions was used to generate individual scores of perceived social support score for each individual resource, taking the participant's evaluation of it into account. A social support variable was then calculated as the mean of these three scores (or two if a participant did not have a partner at the time of the interview).

\section{Negative life events}

The presence of negative life events in the previous 12 months was measured at baseline. Negative life events were based on the Brugha life events categories (Brugha et al. 1985) and included 10 different events, for example death of a relative, divorce and 
major financial difficulties. The numbers of events were categorized as 0, 1 and more than 1 , in order to construct three approximately equal groups.

\section{Statistical analyses}

Statistical analyses were performed using Stata version 13 (StataCorp. 2013). Only participants with complete data were analysed. First, baseline characteristics of all dependent and independent variables were assessed for the complete sample. Moreover, subjects with clinically validated PE at baseline were compared with subjects without clinically validated PE using chi-square tests and independent sample t-tests.

Second, logistic regression analyses were performed in the complete sample using self-reported or clinically validated PE as the independent variable and the different outcomes (i.e. physical violence, psychological violence or arrest) as the dependent variables. The independent variables were divided into hallucinations and delusions in separate analyses. Logistic regression analyses were performed in four different models with varying levels of adjustment. In the first model, the unadjusted associations between the various exposures and outcome measures were examined. The second model added sociodemographic characteristics as independent variables. In the third model, associations were adjusted for sociodemographic characteristics and dimensional symptom scales for substance abuse, bipolar disorder, major depression/dysthymia, anxiety disorders, ADHD, ODD, conduct disorder, antisocial personality. Before adding the symptom scores, linearity of the associations was assessed by adding both the linear and the quadratic term of the symptoms scale in separate logistic regression analyses. If the quadratic term reached statistical significance, both the quadratic and the linear term were used; if the quadratic term did not reach significance, only the linear terms were used. In the fourth and final model, contextual factors including childhood trauma, negative life events and social support were added to the regression models.

Third, dose-response associations between PE and various outcomes were examined by repeating analyses using a continuous scale of PE as the independent variable. Finally, confounding by presence of categorical mental disorder was examined by repeating all analyses stratified by presence or absence of any baseline mental disorder.

\section{Results}

\section{Baseline characteristics}

Of the included participants, $10.4 \%(\mathrm{~N}=630)$ had self-reported PE and 6.2\% ( $N=362)$ had clinically validated $P E$, after exclusion of individuals with psychotic disorder $(n=43)$. Compared with the group without clinically validated PE, the group with clinically validated PE was significantly younger, had a smaller proportion of males and higher scores 
on all symptom scales (Table 1). Moreover, at baseline, lifetime prevalence of both physical and psychological violence perpetration towards intimate partners was higher among individuals with PE; physical violence perpetration towards children was more prevalent as well. In addition, individuals with PE had significantly more negative life events, including childhood trauma, and experienced less social support.

\section{Physical violence}

Incidence of physical violence was $1.3 \%(\mathrm{~N}=36)$. Individuals with any self-reported or clinically validated PE were at increased risk of physical violence with odds ratios (OR) of 2.63 (95\% Cl 1.15 - 6.03; self-reported PE) and 2.80 (95\% Cl 1.08 - 7.30; clinically validated PE), respectively (Table 2). Dividing PE into hallucinations and delusions revealed that delusions were not associated with an increased risk of violence. On the other hand, both self-reported and clinically validated hallucinations at baseline were associated with physical violence at follow-up. Adjusting the analyses for demographic characteristics (model 2) increased the ORs. Further adjustment for dimensional symptom scales (model 3) slightly decreased the associations, but hallucinations remained associated with an increased risk of physical violence. In the final model, associations further decreased. However, self-reported $(\mathrm{OR}=2.83,95 \% \mathrm{Cl} 1.05-7.65)$ and clinically validated hallucinations $(\mathrm{OR}=3.59,95 \% \mathrm{Cl} 1.09$ - 11.81) remained significantly associated with physical violence perpetration.

Continuously defined PE were similarly associated with physical violence perpetration as dichotomously defined PE. In the fully adjusted model, the risk of physical violence perpetration increased in a dose-response fashion as the number of clinically validated hallucinations increased $(\mathrm{OR}=2.71,95 \% \mathrm{Cl} 1.39-5.28)$, but there was no association between number of delusions and physical violence perpetration.

In the subsample without baseline mental disorder, there was no association between PE and physical violence perpetration (Table 2). However, in the subgroup of individuals with any baseline mental disorder, individuals with PE were at increased risk of physical violence perpetration. Analysing hallucinations and delusions separately revealed that both self-reported hallucinations (OR=4.49, 95\% Cl $1.37-17.73$ ) and clinically validated hallucinations $(\mathrm{OR}=5.22,95 \% \mathrm{Cl} 1.22-22.37)$ were associated with physical violence perpetration, even after adjustment for demographics, dimensional psychopathology and contextual confounders (Table 2). Similar to the results in the complete sample, there was no association between delusions and physical violence perpetration in the subgroup of individuals with any baseline mental disorder. 


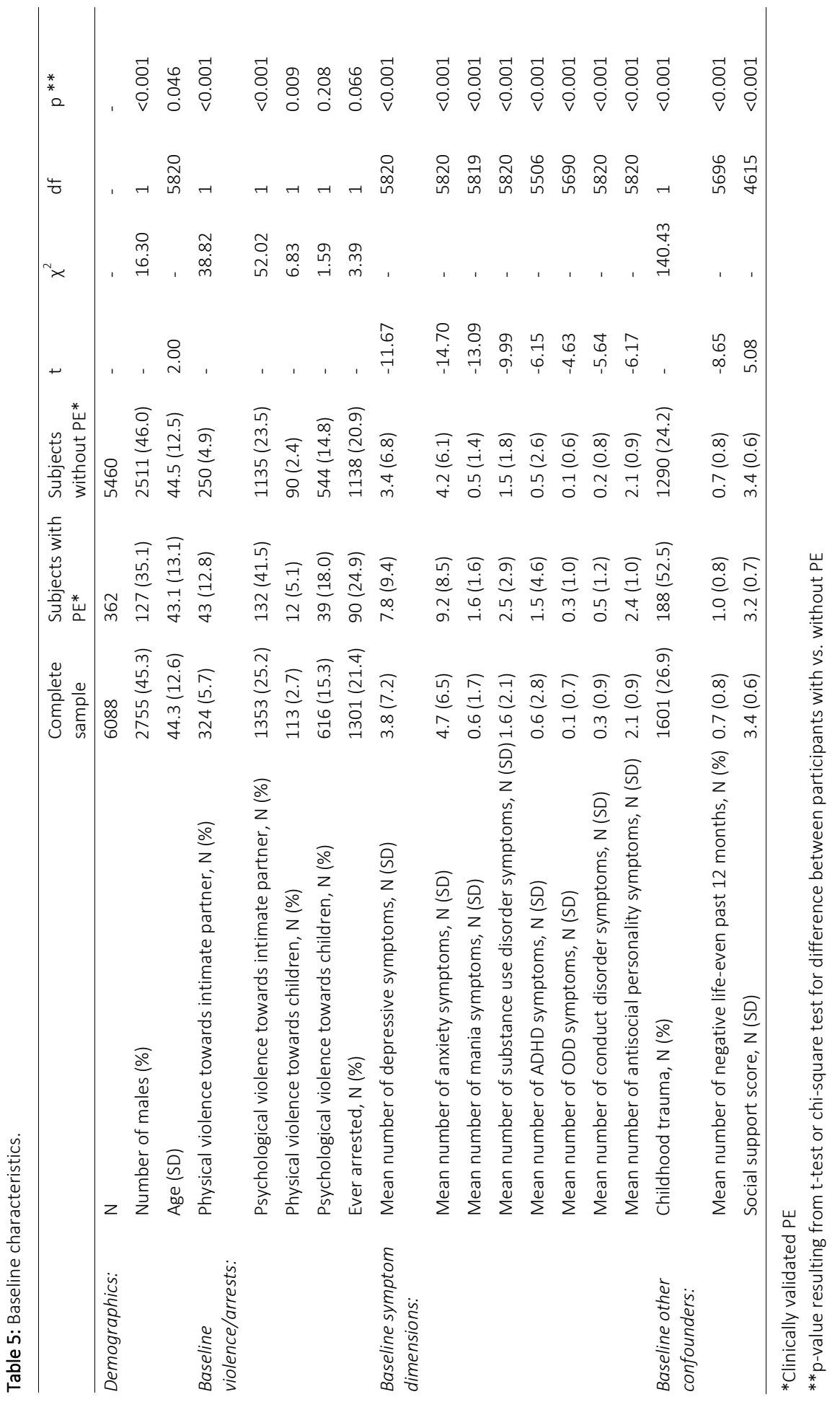


Table 6: Results (odds ratios and 95\% Cl) from logistic regression analyses on the association between selfreported (SR) and clinically validated (CV) psychotic experiences and six-year incidence of physical violence perpetration in a general population sample and two subsamples.

\begin{tabular}{|c|c|c|c|c|}
\hline & \multicolumn{4}{|c|}{ Incident physical violence } \\
\hline & Model $1^{\mathrm{a}}$ & Model $2^{b}$ & Model $3^{\mathrm{c}}$ & Model $4^{d}$ \\
\hline & \multicolumn{4}{|c|}{ Complete sample (excluding baseline psychotic disorder; $\mathrm{N}=3283$ ) } \\
\hline$S R$ Any PE & $2.63(1.15-6.03)^{*}$ & $2.27(0.91-5.65)$ & $1.83(0.67-5.04)$ & $1.54(0.55-4.37)$ \\
\hline Any delusion & $2.34(0.82-6.70)$ & $1.64(0.48-5.55)$ & $1.06(0.27-4.23)$ & $0.93-0.23-3.74)$ \\
\hline Any hallucination & $3.50(1.44-8.50)^{* *}$ & $3.75(1.49-9.47)^{* *}$ & $3.37(1.28-8.90)^{*}$ & $2.83(1.05-7.65)^{*}$ \\
\hline \multirow[t]{4}{*}{ CV Any PE } & $2.80(1.08-7.30)^{*}$ & $2.96(1.11-7.91)^{*}$ & $2.44(0.83-7.19)$ & $2.13(0.71-6.41)$ \\
\hline & $1.51(0.36-6.37)$ & $1.59(0.37-6.87)$ & $1.14(0.23-5.59)$ & $1.04(0.21-5.27)$ \\
\hline & $3.76(1.30-10.86)^{*}$ & $4.42(1.48-13.20)^{* *}$ & $4.34(1.36-13.86)^{*}$ & $3.59(1.09-11.81)^{*}$ \\
\hline & \multicolumn{4}{|c|}{ Subsample of individuals without any baseline mental disorder $(N=2014)$} \\
\hline$S R$ Any PE & $1.11(0.15-8.46)$ & $1.61(0.20-12.81)$ & $1.25(0.13-11.64)$ & $1.00(0.10-10.15)$ \\
\hline Any delusion & N.A. & N.A. & N.A. & N.A. \\
\hline Any hallucination & $1.73(0.23-13.29)$ & $2.27(0.28-18.48)$ & $1.72(0.17-17.30)$ & $1.34(0.12-14.98)$ \\
\hline \multirow{3}{*}{$\begin{array}{l}\text { CV Any PE } \\
\text { Any delusion } \\
\text { Any hallucination }\end{array}$} & $1.76(0.23-13.49)$ & $2.96(0.36-23.99)$ & $1.88(0.19-19.07)$ & $1.55(0.14-17.58)$ \\
\hline & N.A. & N.A. & N.A. & N.A. \\
\hline & $2.83(0.37-21.89)$ & $4.90(0.59-40.97)$ & $3.08(0.26-36.80)$ & $2.37(0.18-32.11)$ \\
\hline
\end{tabular}
Subsample of individuals with any baseline mental disorder (excluding baseline psychotic disorder, $\mathrm{N}=1269$ )

\begin{tabular}{lllll} 
SR Any PE & $2.78(1.07-7.25)^{*}$ & $2.57(0.88-7.48)$ & $2.84(0.89-9.00)$ & $2.36(0.72-7.75)$ \\
$\quad \begin{array}{l}\text { Any delusion } \\
\text { Any hallucination }\end{array}$ & $2.57(0.85-7.77)$ & $2.00(0.56-7.22)$ & $2.06(0.52-8.27)$ & $1.75(0.43-7.12)$ \\
CV Any PE & $2.64(0.86-8.07)$ & $2.99(0.94-9.54)$ & $3.48(0.99-12.29)$ & $3.03(0.84-11.00)$ \\
$\quad$ Any delusion & $1.63(0.37-7.20)$ & $1.84(0.41-8.38)$ & $1.82(0.35-9.39)$ & $1.58(0.29-8.42)$ \\
$\quad$ Any hallucination & $3.44(0.97-12.16)$ & $4.61(1.22-17.38)^{*}$ & $6.15(1.48-25.60)^{*}$ & $5.22(1.22-22.37)^{*}$ \\
\hline
\end{tabular}

$* \mathrm{p}<0.05, * * \mathrm{p}<0.01$

a Model 1: Unadjusted

${ }^{b}$ Model 2: Adjusted for demographics (gender, age, ethnicity, education, employment, socioeconomic status)

${ }^{c}$ Model 3: Adjusted for demographics and dimensional psychopathology (substance abuse, bipolar disorder, major depression, dysthymia, anxiety disorders, ADHD, ODD, conduct disorder, antisocial personality)

${ }^{d}$ Model 4: Adjusted for demographics, dimensional psychopathology, contextual factors (childhood trauma, social support, negative life events)

\section{Psychological violence}

Incidence of psychological violence was $20.1 \%$ ( $N=265)$. In the complete sample, both delusions and hallucinations at baseline were associated with an increased risk of psychological violence perpetration at follow-up (Table 3). Adjusting the analyses for demographical characteristics did not change the results (model 2). However, correcting for dimensional psychopathology (model 3 ) decreased the associations and only clinically validated hallucinations remained significantly associated with psychological violence perpetration. However, inclusion of contextual confounders in the final model further decreased the ORs and no association remained. Results of the analyses using a contin- 
uous scale of PE were similar to the results using dichotomously defined PE as the independent variable.

In the subsample of individuals without any mental disorder at baseline, selfreported delusions were associated with an increased risk of psychological violence perpetration (Table 3). However, in the fully adjusted model, self-reported delusions were no longer associated with an increased risk of psychological violence perpetration, neither was there an association between clinically validated delusions and psychological violence perpetration. Results of the analyses in the subsample of individuals with any baseline mental disorder were similar to the results in the complete sample.

Table 3: Results (odds ratios and 95\% Cl) from logistic regression analyses on the association between selfreported (SR) and clinically validated (CV) psychotic experiences and six-year incidence of psychological violence in a general population sample and two subsamples.

\begin{tabular}{|c|c|c|c|c|}
\hline & \multicolumn{4}{|c|}{ Incident psychological violence } \\
\hline & Model $1^{\text {a }}$ & Model $2^{b}$ & Model $3^{\mathrm{c}}$ & Model $4^{d}$ \\
\hline & \multicolumn{4}{|c|}{ Complete sample (excluding baseline psychotic disorder; $N=3283$ ) } \\
\hline$S R$ Any PE & $1.91(1.33-2.73)^{* *}$ & $2.18(1.47-3.23)^{* *}$ & $1.51(0.94-2.41)$ & $1.21(0.74-1.97)$ \\
\hline Any delusion & $2.13(1.38-3.29)^{* *}$ & $2.35(1.47-3.74)^{* *}$ & $1.61(0.92-2.81)$ & $1.34(0.76-2.38)$ \\
\hline Any hallucination & $1.97(1.29-2.99)^{* *}$ & $2.13(1.34-3.39)^{* *}$ & $1.52(0.88-2.60)$ & $1.17(0.67-2.06)$ \\
\hline CV Any PE & $1.91(1.25-2.91)^{* *}$ & $2.37(1.50-3.76)^{* *}$ & $1.74(1.01-3.00)^{*}$ & $1.40(0.79-2.48)$ \\
\hline delusion & $1.63(0.96-2.77)$ & $2.00(1.13-3.55)^{*}$ & $1.39(0.69-2.80)$ & $1.20(0.59-2.47)$ \\
\hline \multirow[t]{2}{*}{ Any hallucination } & $2.32(1.40-3.84)^{* *}$ & $2.70(1.55-4.69)^{* *}$ & $2.02(1.06-3.85)^{*}$ & $1.56(0.79-3.06)$ \\
\hline & \multicolumn{4}{|c|}{ Subsample of individuals without any baseline mental disorder $(\mathrm{N}=2014)$} \\
\hline$S R$ Any PE & $1.49(0.72-3.12)$ & $1.84(0.83-4.09)$ & $1.96(0.79-4.84)$ & $1.54(0.59-4.01)$ \\
\hline Any delusion & $2.56(1.01-6.49)^{*}$ & $3.20(1.18-8.65)^{*}$ & $3.50(1.09-11.28)^{*}$ & $3.18(0.96-10.60$ \\
\hline Any hallucination & $1.03(0.38-2.75)$ & $1.17(0.41-3.33)$ & $1.21(0.38-3.89)$ & $0.79(0.22-2.92)$ \\
\hline CV Any PE & $1.10(0.41-2.97)$ & $1.54(0.53-4.48)$ & $2.06(0.65-6$ & $1.56(0.44-5.58)$ \\
\hline Any delusion & $0.85(0.19-3.85)$ & $1.17(0.24-5.75)$ & $1.71(0.32-9.14)$ & $1.76(0.31-9.85)$ \\
\hline \multirow[t]{2}{*}{ Any hallucination } & $1.14(0.32-4.06)$ & $1.65(0.42-6.47)$ & $2.04(0.47-8.89)$ & $1.13(0.19-6.63)$ \\
\hline & $\begin{array}{l}\text { Subsample of individ } \\
\text { disorder, } \mathrm{N}=1269 \text { ) }\end{array}$ & & & \\
\hline SR Any PE & $1.76(1.15-2.69)^{* *}$ & $1.98(1.23-3.18)^{* *}$ & $1.48(0.84-2.61)$ & $1.23(0.68-2.21)$ \\
\hline Any delusion & $1.68(1.02-2.76)^{*}$ & $1.78(1.04-3.05)^{*}$ & $1.47(0.78-2.79)$ & $1.21(0.62-2.33)$ \\
\hline Any hallucination & $2.01(1.24-3.27)^{* *}$ & $2.14(1.25-3.68)^{* *}$ & $1.53(0.81-2.91)$ & $1.31(0.68-2.52)$ \\
\hline CV Any PE & $1.86(1.14-3.02)^{*}$ & $2.26(1.32-3.85)^{* *}$ & $1.77(0.92-3.40)$ & $1.55(0.80-2.98)$ \\
\hline Any delusion & $1.50(0.84-2.68)$ & $1.83(0.97-3.45)$ & $1.57(0.72-3.44)$ & $1.28(0.58-2.83)$ \\
\hline Any hallucination & $2.32(1.30-4.11)^{* *}$ & $2.50(1.34-4.68)^{* *}$ & $1.87(0.88-3.99)$ & $1.74(0.81-3.71)$ \\
\hline \multicolumn{5}{|l|}{$* p<0.05, * * p<0.01$} \\
\hline \multicolumn{5}{|l|}{${ }^{a}$ Model 1: Unadjusted } \\
\hline \multicolumn{5}{|c|}{${ }^{\mathrm{b}}$ Model 2: Adjusted for demographics (gender, age, ethnicity, education, employment, socioeconomic status) } \\
\hline \multicolumn{5}{|c|}{$\begin{array}{l}\text { 'Model 3: Adjusted for demographics and dimensional psychopathology (substance abuse, bipolar disorder, } \\
\text { major depression, dysthymia, anxiety disorders, ADHD, ODD, conduct disorder, antisocial personality) }\end{array}$} \\
\hline
\end{tabular}




\section{Arrest}

Incidence of arrest was $2.6 \%$ ( $\mathrm{N}=99)$. Individuals with self-reported or clinically validated PE at baseline were at increased risk of incident arrest (Table 4). Further assessment of this association showed that delusions were associated with an increased risk of arrest at follow-up, while hallucinations were not. Adjustment for demographics, dimensional psychopathology and contextual confounders slightly decreased the effect sizes, but both self-reported delusions ( $\mathrm{OR}=3.06,95 \% \mathrm{Cl} 1.55-6.03)$ and clinically validated delusions $(\mathrm{OR}=3.24,95 \% \mathrm{Cl} 1.47-7.13)$ remained significantly associated with incident arrest.

Associations between continuously defined PE and arrest were similar to the associations between dichotomously defined PE and arrest. In the fully adjusted model, the risk of arrest increased in a dose-response fashion as the number of clinically validated delusions increased $(\mathrm{OR}=1.57,95 \% \mathrm{Cl} 1.00-2.47)$. There was no association between the number of hallucinations and risk of arrest.

Analyses in the subsample of individuals without any baseline mental disorder showed that both self-reported delusions (OR=6.25, 95\% Cl $2.01-19.44)$ and clinically validated delusions ( $\mathrm{OR}=5.37,95 \% \mathrm{Cl} 1.45-19.79)$ were strongly associated with incident arrest in the fully adjusted model (Table 4). Analyses in the sample with any baseline mental disorder showed similar results, although results were no longer statistically significant in the final model (Table 4).

Table 4: Results (odds ratios and 95\% Cl) from logistic regression analyses on the association between selfreported (SR) and clinically validated (CV) psychotic experiences and three-year incidence of arrest in a general population sample and two subsamples.

\begin{tabular}{|c|c|c|c|c|}
\hline & Incident arrest & & & \\
\hline & Model $1^{\mathrm{a}}$ & Model $2^{b}$ & Model $3^{c}$ & Model $4^{d}$ \\
\hline & \multicolumn{4}{|c|}{ Complete sample (excluding baseline psychotic disorder; $\mathrm{N}=4218$ ) } \\
\hline SR Any PE & $3.16\left(1.96-5.11^{* *}\right.$ & $3.27(1.94-5.51)^{* *}$ & $2.40(1.28-4.49)^{* *}$ & $2.12(1.12-4.01)^{*}$ \\
\hline Any delusion & $4.50(2.70-7.49)^{* *}$ & $4.49(2.57-7.85)^{* *}$ & $3.52(1.79-6.90)^{* *}$ & $3.06(1.55-6.03)^{* *}$ \\
\hline Any hallucination & $1.92(0.98-3.74)$ & $2.01(1.00-4.07)$ & $1.44(0.63-3.29)$ & $1.22(0.52-2.84)$ \\
\hline CV Any PE & $3.07(1.74-5.44)^{* *}$ & $3.65(1.98-6.71)^{* *}$ & $2.47(1.17-5.20)^{*}$ & $2.18(1.03-4.64)^{*}$ \\
\hline Any delusion & $4.04(2.20-7.44)^{* *}$ & $4.84(2.51-9.33)^{* *}$ & $3.71(1.70-8.14)^{* *}$ & $3.24(1.47-7.13)^{* *}$ \\
\hline \multirow[t]{2}{*}{ Any hallucination } & $1.75(0.75-4.07)$ & $2.06(0.85-4.99)$ & $1.03(0.33-3.21)$ & $0.86(0.27-2.73)$ \\
\hline & \multicolumn{4}{|c|}{ Subsample of individuals without any baseline mental disorder $(\mathrm{N}=2536)$} \\
\hline SR Any PE & $3.47(1.51-7.98)^{* *}$ & $3.62(1.37-9.54)^{* *}$ & $3.43(1.18-9.98)^{*}$ & $2.76(0.93-8.23)$ \\
\hline Any delusion & $6.59(2.82-15.38)^{* *}$ & $8.06(2.96-21.99) * *$ & $7.48(2.46-22.78)^{* *}$ & *6.25 (2.01 - 19.44)** \\
\hline Any hallucination & $0.68(0.09-4.98)$ & $0.69(0.09-5.44)$ & $0.65(0.07-5.76)$ & $0.43(0.04-4.20)$ \\
\hline CV Any PE & $3.23(1.12-9.31)^{*}$ & $4.31(1.35-13.76)^{*}$ & $4.28(1.25-14.67)^{*}$ & $3.30(0.92-11.81)$ \\
\hline Any delusion & $5.62(1.91-16.48)^{* *}$ & $8.23(2.48-27.34)^{* *}$ & $6.96(1.98-24.54)^{* *}$ & *5.37 (1.45 - 19.79)* \\
\hline Any hallucination & $1.10(0.15-8.15)$ & $1.28(0.15-10.62)$ & $1.15(0.11-11.87)$ & $0.79(0.07-9.15)$ \\
\hline
\end{tabular}




\begin{tabular}{|c|c|c|c|c|}
\hline & \multicolumn{4}{|l|}{ Incident arrest } \\
\hline & Model $1^{\mathrm{a}}$ & Model $2^{b}$ & Model $3^{c}$ & Model $4^{d}$ \\
\hline & \multicolumn{4}{|c|}{$\begin{array}{l}\text { Subsample of individuals with any baseline mental disorder (excluding baseline psychotic } \\
\text { disorder, } \mathrm{N}=1682 \text { ) }\end{array}$} \\
\hline$S R$ Any PE & $2.48(1.36-4.49)^{* *}$ & $2.37(1.25-4.49)^{* *}$ & $1.98(0.89-4.38)$ & $1.81(0.80-4.08)$ \\
\hline Any delusion & $3.04(1.60-5.78)^{* *}$ & $2.70(1.36-5.35)^{* *}$ & $2.50(1.07-5.87)^{*}$ & $2.25(0.94-5.36)$ \\
\hline Any hallucination & $2.00(0.96-4.18)$ & $1.97(0.90-4.28)$ & $1.72(0.68-4.37)$ & $1.55(0.60-3.99)$ \\
\hline CV Any PE & $2.42(1.22-4.81)^{*}$ & $2.59(1.25-5.37)^{*}$ & $1.95(0.77-4.96)$ & $1.86(0.72-4.77)$ \\
\hline Any delusion & $2.80(1.33-5.91)^{* *}$ & $3.09(1.39-6.83)^{* *}$ & $2.88(1.06-7.80)^{*}$ & $2.72(1.00-7.44)$ \\
\hline Any hallucination & $1.63(0.63-4.20)$ & $1.73(0.64-4.67)$ & $0.92(0.24-3.54)$ & $0.84(0.21-3.28)$ \\
\hline \multicolumn{5}{|l|}{$* p<0.05, * * p<0.01$} \\
\hline \multicolumn{5}{|c|}{ a'Model 1: Unadjusted } \\
\hline \multicolumn{5}{|c|}{ bodel 2: Adjusted for demographics (gender, age, ethnicity, education, employment, socioeconomic status) } \\
\hline \multicolumn{5}{|c|}{$\begin{array}{l}\text { 'Model 3: Adjusted for demographics and dimensional psychopathology (substance abuse, bipolar disorder, } \\
\text { major depression, dysthymia, anxiety disorders, ADHD, ODD, conduct disorder, antisocial personality) }\end{array}$} \\
\hline
\end{tabular}

\section{Discussion}

\section{Overview of results}

The present study is the first to investigate the longitudinal association between both self-reported and clinically validated PE on the one hand, and incident physical violence, psychological violence and arrest on the other, in a prospective, general population cohort. Participants with hallucinations at baseline were at increased risk of perpetrating physical violence over the follow-up period of six years, even after adjustment for demographics, dimensional psychopathology and contextual confounders. Delusions were not associated with an increased risk of physical violence in the complete sample. On the other hand, both delusions and hallucinations were associated with an increased risk of psychological violence in the unadjusted analyses. However, adjustment for dimensional psychopathology decreased all effect sizes and rendered them statistically nonsignificant, indicating that the association between PE and psychological violence can be explained by the presence of non-psychotic psychopathology. The finding that PE in the subsample without any mental disorder at baseline did not predict psychological violence, even in the unadjusted analyses, supports this explanation. Finally, the presence of delusions at baseline was associated with an increased risk of reporting arrest, after controlling for demographics, dimensional psychopathology and contextual risk factors. Hallucinations were not associated with an increased risk of arrest. Analyses using continuous scales of PE showed similar associations as the analyses using dichotomously defined PE. The risk of physical violence perpetration increased as the number of hallucinations increased; the risk of arrest increased as the number of delusions increased. 


\section{Psychotic experiences and physical violence perpetration}

Previous research suggested that a specific subset of delusional symptoms called 'threat/control-override' (TCO) symptoms is associated with violence perpetration, because these symptoms cause the person to believe that people are out to harm them or that outside forces are controlling their minds (Link et al. 1998; Rueve and Welton 2008; Swanson et al. 1996). The present study cannot be considered as a replication, as the results showed that hallucinations were associated with an increased risk of physical violence perpetration, while delusions were not associated with violence perpetration. This finding is consistent with previous studies in the general population that also reported associations between hallucinations and physical violence perpetration (Nederlof et al. 2012; Mojtabai 2006; Kinoshita et al. 2011). However, a recent metaanalysis of seven cross-sectional population surveys found no association between hallucinations and violence; only paranoid ideation was associated with an increased risk of violence perpetration (Coid et al. 2016). In the present study, delusions predicted violence perpetration only in the subsample with any baseline mental disorder, although results were statistically non-significant. Thus, PE in the context of a mental disorder may be differently associated with physical violence than PE in absence of a mental disorder. However, more research is required to replicate this finding. Moreover, a previous prospective study showed that delusions among psychiatric inpatients after discharge did not predict future violence, unless there was a close temporal relationship between delusional ideation and violence (Ullrich et al. 2014). In the present analyses, this temporal proximity between PE and violence could not be taken into account since at baseline only lifetime PE were assessed and at follow-up only violence in the six years before the interview was assessed. This may explain why delusions were not associated with future physical violence in the analyses in the complete sample.

Although the association between PE and physical violence perpetration has been confirmed in various cross-sectional studies, the mechanism behind this association remains unclear. The association between PE and violence may be the result of confounding by other variables. Previous research showed that the association between PE and auto-aggressive behaviour (i.e. suicidal behaviour and self-injury) was confounded by co-occurring non-psychotic psychopathology (Honings et al. 2016), thus making it likely that this also applies to hetero-aggression (i.e. interpersonal violence), as confirmed in the study by Coid et al. (2016). The present analyses were adjusted for demographics, dimensional non-psychotic psychopathology and several contextual confounders identified in previous studies (ten Have et al. 2014), which included childhood trauma, negative life events and social support. Consistent with previous research (ten Have et al. 2014), adjustment for these variables decreased effect sizes. This indicates that the associations are at least in part confounded. Inclusion of other variables that are associated with both PE and violence perpetration in the regression models, for 
example stress reactivity (Myin-Germeys et al. 2001), anger (Nederlof et al. 2012) or impulsivity (Krakowski and Czobor 2014) might have further decreased effect sizes.

Moreover, the present analyses were only adjusted for baseline non-psychotic psychopathology. However, individuals with PE are at increased risk to develop a mental disorder (Linscott and van Os 2013; Kelleher et al. 2012b; Kaymaz et al. 2012) and, thus, the reported association between hallucinations and violence perpetration can be explained by non-psychotic psychopathology after baseline. In other words, PE and nonpsychotic psychopathology may lie on the causal pathway towards the outcome of violence or arrest. To examine this, sensitivity analyses were conducted, which showed that adjustment for incident mental disorders did not decrease ORs. On the other hand, ORs increased when a sensitivity analysis was conducted in the subsample of individuals with incident mental disorders. This finding is consistent with the main results in table 2 that showed that hallucinations were particularly predictive of violence perpetration in the context of a baseline mental disorder. Thus, the association between hallucinations and violence perpetration possibly reflects the increased risk of violence perpetration in individuals in distress in the context of a mental disorder. This is in agreement with previous studies that demonstrated that PE serve as an indicator of illness severity and poor outcome in persons with non-psychotic psychopathology (Wigman et al. 2012; Wigman et al. 2014; Perlis et al. 2011; Guloksuz et al. 2015; Kelleher et al. 2012b).

In addition, it is possible that the association between hallucinations and violence perpetration reflects the association between one specific PE, for example auditory hallucinations, and violence perpetration, since previous, cross-sectional work found an association between command hallucinations and violence perpetration (McNiel et al. 2000). More longitudinal research with larger sample sizes is required to investigate this question, since statistical power in the current dataset is too low to assess associations between specific PE and violence perpetration.

\section{Psychotic experiences and psychological violence}

The present study provides the first evidence that PE in the general population are prospectively associated with an increased risk of psychological violence perpetration. However, after adjustment for dimensional psychopathology, no association remained, indicating that the association between PE and psychological violence is non-specific and reflects the increased risk of psychological violence in individuals with nonpsychotic psychopathology (ten Have et al. 2014) that is prevalent among individuals with PE (van Os et al. 2000; DeVylder et al. 2014; Kelleher et al. 2012b).

\section{Psychotic experiences and arrest}

Incident arrest was more frequent in individuals experiencing delusions than in individuals without delusions. This result was found in both the complete sample and the sub- 
sample without baseline mental disorders, even in the most adjusted models. Contrary to the association found between hallucinations and physical violence perpetration, there was no association between hallucinations and arrest. As far as we are aware, only one previous study assessed the association between PE and arrest, and showed that PE were associated with an increased risk of arrest for both aggravated assault and other illegal behaviours (Mojtabai 2006). On the other hand, previous studies showed that the prevalence of psychotic disorder is much higher among jail detainees than among general population samples in the same age group (Teplin 1990), indicating that there is an association between full blown psychosis and arrest. Similarly, Vreugdenhil et al. (2004) showed that $34 \%$ of a sample of incarcerated boys reported any psychotic symptom. Our results indicate that low grade PE, as part of the extended psychosis phenotype (van Os et al. 2000), are also associated with an increased risk of arrest. The finding that delusions predict arrest is consistent with previous work that demonstrated that paranoid ideation is associated with violent incidents involving the police (Coid et al. 2016).

Sensitivity analyses were conducted to examine the mediating effect of incident mental disorders on the association between delusions and arrest. Results showed that inclusion of incident mental disorders in the regression models increased ORs. Moreover, ORs increased further when sensitivity analyses were conducted in the subsample without incident mental disorders. This result is consistent with the main results in table 4 that showed that delusions were particularly predictive of arrest in the absence of a mental disorder. It is unclear why hallucinations are particularly predictive of violence perpetration in the context of a mental disorder, while delusions are particularly predictive of arrest in the absence of a mental disorder. Moreover, it remains unclear why physical violence perpetration is predicted by hallucinations, while arrest is best predicted by delusions. A possible explanation is that the outcome of arrest does not only include arrests for physical violence, but also for other behaviours or criminal offenses that may be associated with delusions instead of hallucinations - for example social conflicts in the context of disordered behaviour arising from paranoid or grandiose delusions. More longitudinal research is needed to replicate the results of the present study and examine underlying mechanisms.

\section{Self-reported versus clinically validated psychotic experiences}

The results of this study show that both self-reported and clinically validated PE are predictive of incident physical violence, psychological violence and arrest. In general, ORs for the associations between clinically validated PE and the various outcomes were slightly larger than effect sizes for self-reported PE. This finding is consistent with previous work that showed that clinically validated PE were stronger associated with various mental health outcomes than self-reported PE (van Nierop et al. 2012). 


\section{Strengths and limitations}

Strength of the present study is the prospective, longitudinal study design that enables the assessment of the temporal sequence of the association between PE and violence, while previous studies were cross-sectional. Another strength is the use of both selfreported and clinically validated PE. Previous studies on the association between PE and violence only included self-reported PE as exposure. A final strength is the use of dimensional symptom scales to adjust the regression models, rather than using a narrow, dichotomous definition of mental disorder that leaves room for residual confounding.

The results of the present study should be interpreted in the light of some methodological limitations. First, the outcome of physical violence was rare. Even though the study included 6646 individuals at baseline, statistical power was low, as shown by the wide 95\% confidence intervals. For that reason, it was impossible to assess the association between individual PE items and the various outcomes. Second, the questions used to assess physical violence and psychological violence at follow-up were not identical to the questions used at baseline, since at baseline only violence towards an intimate partner or children was assessed. To overcome this limitation, individuals who never had a partner or children at baseline were excluded from the analyses for the violence outcomes. However, violence perpetration against anyone other than a partner or child could still be missed at baseline. Fortunately, the assessment of baseline physical violence could be improved by integrating an additional question from the screening section of the CIDI. Moreover, research shows that violence perpetration often targets intimate partners (Garcia-Moreno et al. 2006). It is therefore unlikely that the discrepancy between the baseline and follow-up assessments of violence perpetration influences the results to a great extent. Third, not every dimensional psychopathology scale could be generated for each participant, since impulse-control disorders were only assessed in subjects aged 18-44 years and other mental disorders only if a participant answered positively to a key question for the respective disorder in the screening section. This may have resulted in an overestimation of reported associations. Finally, follow-up data was missing for a proportion of the cohort (31\%). Attrition at follow-up was not associated with baseline mental health status (de Graaf et al. 2013), PE or any violence perpetration. However, baseline arrest was weakly associated with an increased risk of participant attrition $(\mathrm{OR}=1.23,95 \% \mathrm{Cl} 1.09-1.40)$. Since individuals with baseline arrest were also at increased risk for violence perpetration, this may have resulted in more conservative rather than spurious associations.

\section{Future research}

The present study provides fresh evidence that both self-reported and clinically validated PE in general population individuals are associated with an increased risk of future violence perpetration and arrest. However, more longitudinal research with larger sam- 
ples sizes is required to confirm these findings. The use of larger samples offers the opportunity to assess the association between individual PE and violence and arrest and, thus, to examine whether there is any association between the aforementioned TCO symptoms and perpetration of violence in the general population. The results showed that self-reported PE can be used as a valid measure to study these associations. Moreover, future research should take the temporal proximity of exposures and outcomes into account in order to assess whether this changes the associations between PE and physical violence, psychological violence and arrest as reported in this study. 


\section{References}

Brennan, P. A., Mednick, S. A. \& Hodgins, S. 2000. Major mental disorders and criminal violence in a Danish birth cohort. Arch Gen Psychiatry, 57, 494-500.

Brugha, T., Bebbington, P., Tennant, C. \& Hurry, J. 1985. The List of Threatening Experiences: a subset of 12 life event categories with considerable long-term contextual threat. Psychol Med, 15, 189-94.

Coid, J. W., Ullrich, S., Bebbington, P., Fazel, S. \& Keers, R. 2016. Paranoid Ideation and Violence: Metaanalysis of Individual Subject Data of 7 Population Surveys. Schizophr Bull, 42, 907-15.

De Graaf, R., Bijl, R. V., Ten Have, M., Beekman, A. T. \& Vollebergh, W. A. 2004. Rapid onset of comorbidity of common mental disorders: findings from the Netherlands Mental Health Survey and Incidence Study (NEMESIS). Acta Psychiatr Scand, 109, 55-63.

De Graaf, R., Ten Have, M. \& Van Dorsselaer, S. 2010. The Netherlands Mental Health Survey and Incidence Study-2 (NEMESIS-2): design and methods. Int J Methods Psychiatr Res, 19, 125-41.

De Graaf, R., Van Dorsselaer, S., Tuithof, M. \& Ten Have, M. 2013. Sociodemographic and psychiatric predictors of attrition in a prospective psychiatric epidemiological study among the general population. Result of the Netherlands Mental Health Survey and Incidence Study-2. Compr Psychiatry, 54, 1131-9.

Devylder, J. E., Burnette, D. \& Yang, L. H. 2014. Co-occurrence of psychotic experiences and common mental health conditions across four racially and ethnically diverse population samples. Psychol Med, 44, 3503-13.

Douglas, K. S., Guy, L. S. \& Hart, S. D. 2009. Psychosis as a risk factor for violence to others: a meta-analysis. Psychol Bull, 135, 679-706.

Dowden, C. \& Brown, S. L. 2002. The role of substance abuse factors in predicting recidivism: A meta-analysis. Psychology Crime \& Law, 8, 243-264.

Fayyad, J., De Graaf, R., Kessler, R., Alonso, J., Angermeyer, M., Demyttenaere, K., De Girolamo, G., Haro, J. M., Karam, E. G., Lara, C., Lepine, J. P., Ormel, J., Posada-Villa, J., Zaslavsky, A. M. \& Jin, R. 2007. Cross-national prevalence and correlates of adult attention-deficit hyperactivity disorder. Br J Psychiatry, 190, 402-9.

Fazel, S. \& Danesh, J. 2002. Serious mental disorder in 23000 prisoners: a systematic review of 62 surveys. Lancet, 359, 545-50.

Fazel, S., Gulati, G., Linsell, L., Geddes, J. R. \& Grann, M. 2009. Schizophrenia and violence: systematic review and meta-analysis. PLoS Med, 6, e1000120.

Fazel, S., Lichtenstein, P., Grann, M., Goodwin, G. M. \& Langstrom, N. 2010. Bipolar disorder and violent crime: new evidence from population-based longitudinal studies and systematic review. Arch Gen Psychiatry, 67, 931-8.

Fazel, S., Wolf, A., Chang, Z., Larsson, H., Goodwin, G. M. \& Lichtenstein, P. 2015. Depression and violence: a Swedish population study. Lancet Psychiatry, 2, 224-32.

Garcia-Moreno, C., Jansen, H. A., Ellsberg, M., Heise, L., Watts, C. H., Health, W. H. O. M.-C. S. O. W. S. \& Domestic Violence against Women Study, T. 2006. Prevalence of intimate partner violence: findings from the WHO multi-country study on women's health and domestic violence. Lancet, 368, 1260-9.

Guloksuz, S., Van Nierop, M., Lieb, R., Van Winkel, R., Wittchen, H. U. \& Van Os, J. 2015. Evidence that the presence of psychosis in non-psychotic disorder is environment-dependent and mediated by severity of non-psychotic psychopathology. Psychol Med, 45, 2389-401.

Haro, J. M., Arbabzadeh-Bouchez, S., Brugha, T. S., De Girolamo, G., Guyer, M. E., Jin, R., Lepine, J. P., Mazzi, F., Reneses, B., Vilagut, G., Sampson, N. A. \& Kessler, R. C. 2006. Concordance of the Composite International Diagnostic Interview Version 3.0 (CIDI 3.0) with standardized clinical assessments in the WHO World Mental Health surveys. Int J Methods Psychiatr Res, 15, 167-80.

Hodgins, S. \& Janson, C. G. 2002. Criminality and Violence among the Mentally Disordered: The Stockholm Metropolitan Project, Cambridge University Press.

Honings, S., Drukker, M., Groen, R. \& Van Os, J. 2016. Psychotic experiences and risk of self-injurious behaviour in the general population: a systematic review and meta-analysis. Psychol Med, 46, 237-51.

Huang, Y., Kotov, R., De Girolamo, G., Preti, A., Angermeyer, M., Benjet, C., Demyttenaere, K., De Graaf, R., Gureje, O., Karam, A. N., Lee, S., Lepine, J. P., Matschinger, H., Posada-Villa, J., Suliman, S., Vilagut, G. \& 
Kessler, R. C. 2009. DSM-IV personality disorders in the WHO World Mental Health Surveys. Br J Psychiatry, 195, 46-53.

Joyal, C., Dubreucq, J.-L., Gendron, C. \& Millaud, F. 2007. Major Mental Disorders and Violence: A Critical Update. Current Psychiatry Reviews, 3, 33-50.

Kaymaz, N., Drukker, M., Lieb, R., Wittchen, H. U., Werbeloff, N., Weiser, M., Lataster, T. \& Van Os, J. 2012. Do subthreshold psychotic experiences predict clinical outcomes in unselected non-help-seeking populationbased samples? A systematic review and meta-analysis, enriched with new results. Psychol Med, 42, 2239-53.

Kelleher, I., Harley, M., Murtagh, A. \& Cannon, M. 2011. Are screening instruments valid for psychotic-like experiences? A validation study of screening questions for psychotic-like experiences using in-depth clinical interview. Schizophr Bull, 37, 362-9.

Kelleher, I., Connor, D., Clarke, M. C., Devlin, N., Harley, M. \& Cannon, M. 2012a. Prevalence of psychotic symptoms in childhood and adolescence: a systematic review and meta-analysis of population-based studies. Psychol Med, 42, 1857-63.

Kelleher, I., Keeley, H., Corcoran, P., Lynch, F., Fitzpatrick, C., Devlin, N., Molloy, C., Roddy, S., Clarke, M. C., Harley, M., Arseneault, L., Wasserman, C., Carli, V., Sarchiapone, M., Hoven, C., Wasserman, D. \& Cannon, M. 2012b. Clinicopathological significance of psychotic experiences in non-psychotic young people: evidence from four population-based studies. Br J Psychiatry, 201, 26-32.

Kessler, R. C., Molnar, B. E., Feurer, I. D. \& Appelbaum, M. 2001. Patterns and mental health predictors of domestic violence in the United States: results from the National Comorbidity Survey. Int J Law Psychiatry, 24, 487-508.

Kessler, R. C. \& Ustun, T. B. 2004. The World Mental Health (WMH) Survey Initiative Version of the World Health Organization (WHO) Composite International Diagnostic Interview (CIDI). Int J Methods Psychiatr Res, 13, 93-121.

Kessler, R. C., Angermeyer, M., Anthony, J. C., R, D. E. G., Demyttenaere, K., Gasquet, I., G, D. E. G., Gluzman, S., Gureje, O., Haro, J. M., Kawakami, N., Karam, A., Levinson, D., Medina Mora, M. E., Oakley Browne, M. A., Posada-Villa, J., Stein, D. J., Adley Tsang, C. H., Aguilar-Gaxiola, S., Alonso, J., Lee, S., Heeringa, S., Pennell, B. E., Berglund, P., Gruber, M. J., Petukhova, M., Chatterji, S. \& Ustun, T. B. 2007. Lifetime prevalence and age-of-onset distributions of mental disorders in the World Health Organization's World Mental Health Survey Initiative. World Psychiatry, 6, 168-76.

Kinoshita, Y., Shimodera, S., Nishida, A., Kinoshita, K., Watanabe, N., Oshima, N., Akechi, T., Sasaki, T., Inoue, S., Furukawa, T. A. \& Okazaki, Y. 2011. Psychotic-like experiences are associated with violent behavior in adolescents. Schizophr Res, 126, 245-51.

Krakowski, M. I. \& Czobor, P. 2014. Depression and impulsivity as pathways to violence: implications for antiaggressive treatment. Schizophr Bull, 40, 886-94.

Large, M. M. \& Nielssen, O. 2011. Violence in first-episode psychosis: a systematic review and meta-analysis. Schizophr Res, 125, 209-20.

Lenzenweger, M. F., Lane, M. C., Loranger, A. W. \& Kessler, R. C. 2007. DSM-IV personality disorders in the National Comorbidity Survey Replication. Biol Psychiatry, 62, 553-64.

Lewis, D. O., Shanok, S. S., Pincus, J. H. \& Glaser, G. H. 1979. Violent juvenile delinquents: psychiatric, neurological, psychological, and abuse factors. J Am Acad Child Psychiatry, 18, 307-19.

Link, B. G. \& Stueve, A. 1995. Evidence bearing on mental illness as a possible cause of violent behavior. Epidemiol Rev, 17, 172-81.

Link, B. G., Stueve, A. \& Phelan, J. 1998. Psychotic symptoms and violent behaviors: probing the components of "threat/control-override" symptoms. Soc Psychiatry Psychiatr Epidemiol, 33 Suppl 1, S55-60.

Linscott, R. J. \& Van Os, J. 2013. An updated and conservative systematic review and meta-analysis of epidemiological evidence on psychotic experiences in children and adults: on the pathway from proneness to persistence to dimensional expression across mental disorders. Psychol Med, 43, 1133-49.

Loranger, A. W., Sartorius, N., Andreoli, A., Berger, P., Buchheim, P., Channabasavanna, S. M., Coid, B., Dahl, A., Diekstra, R. F., Ferguson, B. \& Et Al. 1994. The International Personality Disorder Examination. The 


\section{Chapter 4}

World Health Organization/Alcohol, Drug Abuse, and Mental Health Administration international pilot study of personality disorders. Arch Gen Psychiatry, 51, 215-24.

Mcniel, D. E., Eisner, J. P. \& Binder, R. L. 2000. The relationship between command hallucinations and violence. Psychiatr Serv, 51, 1288-92.

Miller, E., Breslau, J., Chung, W. J., Green, J. G., Mclaughlin, K. A. \& Kessler, R. C. 2011. Adverse childhood experiences and risk of physical violence in adolescent dating relationships. J Epidemiol Community Health, 65, 1006-13.

Mojtabai, R. 2006. Psychotic-like experiences and interpersonal violence in the general population. Soc Psychiatry Psychiatr Epidemiol, 41, 183-90.

Monahan, J. 1992. Mental disorder and violent behavior. Perceptions and evidence. Am Psychol, 47, 511-21.

Myin-Germeys, I., Van Os, J., Schwartz, J. E., Stone, A. A. \& Delespaul, P. A. 2001. Emotional reactivity to daily life stress in psychosis. Arch Gen Psychiatry, 58, 1137-44.

Nederlof, A. F., Muris, P. \& Hovens, J. E. 2012. Psychotic-like experiences and aggressive behavior in a nonclinical sample. Personality and Individual Differences, 53, 33-37.

Perlis, R. H., Uher, R., Ostacher, M., Goldberg, J. F., Trivedi, M. H., Rush, A. J. \& Fava, M. 2011. Association between bipolar spectrum features and treatment outcomes in outpatients with major depressive disorder. Arch Gen Psychiatry, 68, 351-60.

Rueve, M. E. \& Welton, R. S. 2008. Violence and mental illness. Psychiatry (Edgmont), 5, 34-48.

Skre, I., Onstad, S., Torgersen, S. \& Kringlen, E. 1991. High interrater reliability for the Structured Clinical Interview for DSM-III-R Axis I (SCID-I). Acta Psychiatr Scand, 84, 167-73.

Statacorp. 2013. Stata Statistical Software: Release 13. College Station, TX: StataCorp LP.

Swanson, J. M. 1994. Mental disorder, substance abuse and community violence: an epidemiological approach. Violence and mental disorder. Chicago: University of Chicago Press.

Swanson, J. W., Borum, R., Swartz, M. S. \& Monahan, J. 1996. Psychotic symptoms and disorders and the risk of violent behaviour in the community. Criminal Behaviour and Mental Health, 6, 309-329.

Ten Have, M., De Graaf, R., Van Weeghel, J. \& Van Dorsselaer, S. 2014. The association between common mental disorders and violence: to what extent is it influenced by prior victimization, negative life events and low levels of social support? Psychol Med, 44, 1485-98.

Teplin, L. A. 1990. The Prevalence of Severe Mental Disorder among Male Urban Jail Detainees - Comparison with the Epidemiologic Catchment-Area Program. American Journal of Public Health, 80, 663-669.

Ullrich, S., Keers, R. \& Coid, J. W. 2014. Delusions, anger, and serious violence: new findings from the MacArthur Violence Risk Assessment Study. Schizophr Bull, 40, 1174-81.

Van Dorn, R., Volavka, J. \& Johnson, N. 2012. Mental disorder and violence: is there a relationship beyond substance use? Soc Psychiatry Psychiatr Epidemiol, 47, 487-503.

Van Nierop, M., Van Os, J., Gunther, N., Myin-Germeys, I., De Graaf, R., Ten Have, M., Van Dorsselaer, S., Bak, M. \& Van Winkel, R. 2012. Phenotypically continuous with clinical psychosis, discontinuous in need for care: evidence for an extended psychosis phenotype. Schizophr Bull, 38, 231-8.

Van Os, J., Hanssen, M., Bijl, R. \& Ravelli, A. 2000. Straus (1969) revisited: A psychosis continuum in the general population? Schizophrenia Research, 45, 11-20.

Vreugdenhil, C., Vermeiren, R., Wouters, L. F., Doreleijers, T. A. \& Van Den Brink, W. 2004. Psychotic symptoms among male adolescent detainees in The Netherlands. Schizophr Bull, 30, 73-86.

Wigman, J. T., Van Nierop, M., Vollebergh, W. A., Lieb, R., Beesdo-Baum, K., Wittchen, H. U. \& Van Os, J. 2012. Evidence that psychotic symptoms are prevalent in disorders of anxiety and depression, impacting on illness onset, risk, and severity--implications for diagnosis and ultra-high risk research. Schizophr Bull, 38, 247-57.

Wigman, J. T., Van Os, J., Abidi, L., Huibers, M. J., Roelofs, J., Arntz, A., Kelleher, I. \& Peeters, F. P. 2014. Subclinical psychotic experiences and bipolar spectrum features in depression: association with outcome of psychotherapy. Psychol Med, 44, 325-36.

Williams, J. B., Gibbon, M., First, M. B., Spitzer, R. L., Davies, M., Borus, J., Howes, M. J., Kane, J., Pope, H. G., Jr., Rounsaville, B. \& Et Al. 1992. The Structured Clinical Interview for DSM-III-R (SCID). II. Multisite testretest reliability. Arch Gen Psychiatry, 49, 630-6. 
Psychotic experiences and risk of violence and arrest

Yu, R., Geddes, J. R. \& Fazel, S. 2012. Personality disorders, violence, and antisocial behavior: a systematic review and meta-regression analysis. J Pers Disord, 26, 775-92. 



\section{Chapter}

\section{The interplay of psychosis and victimisation across the life course: a prospective study in the general population}

Steven Honings ${ }^{1}$, Marjan Drukker ${ }^{1}$, Margreet ten Have ${ }^{2}$, Ron de Graaf ${ }^{2}$, Saskia van Dorsselaer ${ }^{2}, \mathrm{Jim}$ van $\mathrm{Os}^{1,3,4}$

1. Department of Psychiatry and Psychology, South Limburg Mental Health Research and Teaching Network, Maastricht University Medical Centre, Maastricht, the Netherlands.

2. Netherlands Institute of Mental Health and Addiction, Utrecht, the Netherlands

3. King's College London, King's Health Partners, Department of Psychosis Studies, Institute of Psychiatry, London, UK

4. Department of Psychiatry, Brain Center Rudolf Magnus Institute, University Medical Center Utrecht, Utrecht, The Netherlands.

Published as:

Honings, S., Drukker, M., Ten Have, M., De Graaf, R., Van Dorsselaer, S. \& Van Os, J. 2017. The interplay of psychosis and victimisation across the life course: a prospective study in the general population. Soc Psychiatry Psychiatr Epidemiol, 52, 1363-1374. 


\begin{abstract}
Background: Psychosis has been associated with adult victimisation. However, it remains unclear whether psychosis predicts incident adult victimisation, or whether adult victimisation predicts incident psychosis. Furthermore, a moderating effect of childhood victimisation on the association between psychosis and adult victimisation has not been investigated.
\end{abstract}

Method: The longitudinal association between baseline psychotic experiences and sixyear incidence of adult victimisation was assessed in a prospective general population cohort of 6646 adults using logistic regression analysis. The association between baseline adult victimisation and six-year incidence of psychotic experiences was examined as well. Furthermore, the moderating effect of childhood victimisation on these bidirectional associations was analysed.

Results: Psychotic experiences and childhood victimisation were both associated with an increased risk of incident adult victimisation. However, this was through competing pathways, as suggested by a negative interaction between psychotic experiences and childhood victimisation. Baseline adult victimisation and childhood victimisation both independently increased the risk of incident psychotic experiences, but there was no interaction between adult victimisation and childhood victimisation.

Conclusion: Psychosis and victimisation are interconnected throughout the life course. Childhood victimisation is connected to psychosis through two pathways: one direct and one indirect through adult victimisation. In individuals without childhood victimisation, psychosis and adult victimisation bidirectionally impact on each other. 


\section{Introduction}

Psychosis has been associated with an increased risk of violence perpetration (Coid et al. 2016; Douglas et al. 2009; Fazel et al. 2009; Fazel and Yu 2011; Large and Nielssen 2011). However, contrary to the common stereotype that individuals with severe mental illness are dangerous (Crisp et al. 2000), evidence shows that these individuals are more likely to be victims of violence than perpetrators of violence (ten Have et al. 2014; Choe et al. 2008). Among individuals with psychosis, victimisation is prevalent (Bebbington et al. 2004), both during childhood (Kelleher et al. 2013; Morgan and Gayer-Anderson 2016; Read et al. 2005) and adulthood (de Mooij et al. 2015; Hart et al. 2012; Kamperman et al. 2014).

Various forms of childhood victimisation, including sexual abuse, physical abuse, emotional abuse (Varese et al. 2012) and being bullied (Wolke et al. 2014), have been associated with psychosis in the literature (Kelleher et al. 2013; Morgan and GayerAnderson 2016; Read et al. 2005). Childhood victimisation has been associated with both psychotic experiences (PE) (Kelleher et al. 2013) and full-blown psychotic disorder (Read et al. 2005; Morgan and Gayer-Anderson 2016), thus covering the complete spectrum of the extended psychosis phenotype (van Os et al. 2000; van Os and Linscott 2012; van Os and Reininghaus 2016). Most research to date has focussed on the hypothesis that childhood victimisation is a risk factor for the development of psychosis (Varese et al. 2012). However, evidence shows that PE increase the risk of incident childhood victimisation as well, thereby showing that the association between childhood victimisation and PE is bidirectional (Kelleher et al. 2013).

Recently, studies found evidence that psychosis is associated with adult victimisation as well. Compared with general population individuals, the prevalence of criminal and violent victimisation among individuals with psychosis and other severe mental illnesses was high (de Mooij et al. 2015; Hart et al. 2012; Kamperman et al. 2014). However, the nature of this association remains unclear, since most studies to date had some methodological limitations. First, most studies to date used cross-sectional study designs to examine the association between psychosis and adult victimisation (de Mooij et al. 2015; Hart et al. 2012; Kamperman et al. 2014). Therefore, it is unclear whether psychosis increases the risk of incident adult victimisation or vice versa. To our knowledge, only one longitudinal study examined the association between adult victimisation and psychosis, showing that adult adversities were associated with an increased risk of incident PE (Lataster et al. 2012). However, no longitudinal study to date examined whether psychosis predicts incident adult victimisation. Thus, it remains unknown whether the association between psychosis and adult victimisation is bidirectional, similar to the association between psychosis and childhood victimisation (Kelleher et al. 2013). Second, few studies examined the influence of childhood victimisation on the association between psychosis and adult victimisation (Khalifeh et al. 2016), while childhood victimisation is associated with an increased risk of both psychosis (Kelleher et al. 2013; Read 
et al. 2005; Morgan and Gayer-Anderson 2016) and adult victimisation (Widom et al. 2008; Barrios et al. 2015; Bensley et al. 2003; Blom et al. 2014; Coid et al. 2001; Mclntyre and Widom 2011; Fry et al. 2012). Previous studies have shown that childhood victimisation and various environmental factors combine synergistically to increase the risk of PE over and above their isolated products (Bebbington et al. 2011; Lataster et al. 2012; Morgan et al. 2014a; Morgan et al. 2014b). However, no previous study examined whether psychosis predicts incident adult victimisation, while simultaneously examining the potential moderating effect of childhood victimisation.

The present study aims to bridge these gaps in the literature. In line with the research on childhood victimisation and PE, we hypothesized that there would be a bidirectional association between $\mathrm{PE}$ and adult victimisation, that is moderated by the presence of childhood victimisation (Fig. 1). More specifically, we hypothesized that: (1) PE are associated with incident adult victimisation; (2) childhood victimisation is associated with incident adult victimisation; (3) the co-occurrence of PE and childhood victimisation predicts a stronger association with incident adult victimisation than the product of their isolated effects; (4) adult victimisation is associated with incident PE; (5) childhood victimisation is associated with incident PE; (6) the co-occurrence of adult victimisation and childhood victimisation predicts a stronger association with incident PE than the product of their isolated effects.

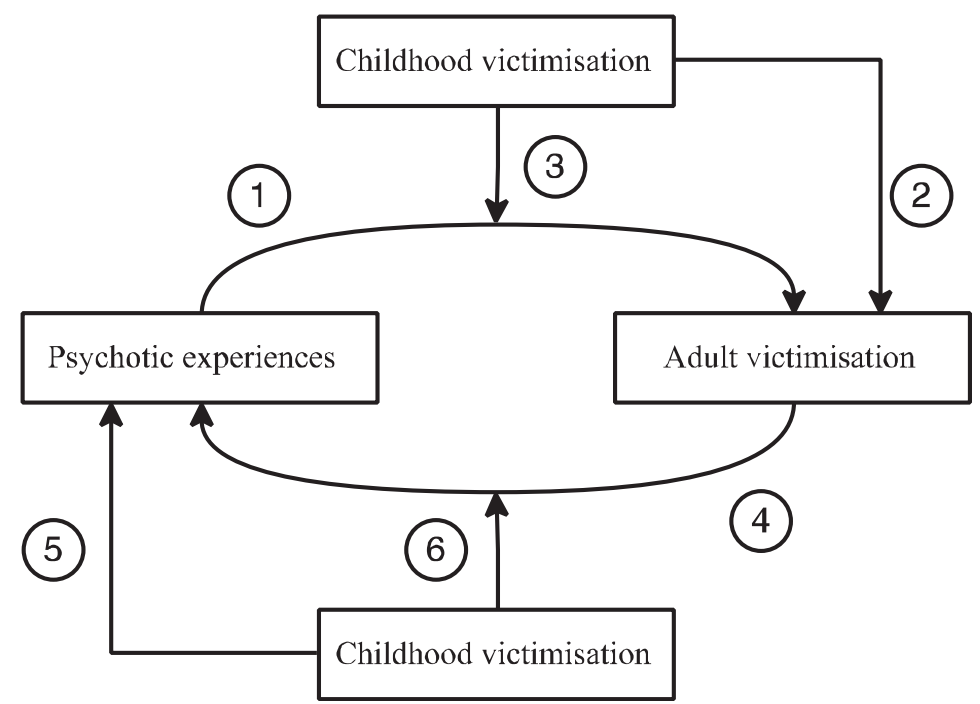

Figure. 1: Hypotheses relating to the bidirectional association between psychotic experiences and victimisation. 


\section{Methods}

\section{Sample}

This study uses data pertaining to the second Netherlands Mental Health Survey and Incidence Study (NEMESIS-2), a longitudinal study of the prevalence, incidence, course and consequences of mental disorders in the Dutch general population (de Graaf et al. 2010). Participants were selected based on a multistage random sampling procedure. At baseline (T0), 6646 persons aged 18-64 years were interviewed with the Composite International Diagnostic Interview (CIDI) version 3.0, a fully structured lay-administered diagnostic interview generating DSM-IV diagnoses (Haro et al. 2006). At follow-up, respectively three $(T 1, N=5503)$ and six years $(T 2, N=4618)$ after baseline, subjects were re-interviewed. A more comprehensive description of the design can be found elsewhere (de Graaf et al. 2010). In the present analyses, only individuals who responded to all three assessments were included.

\section{Psychotic experiences}

PE were assessed at baseline (TO) and both follow-up measurements (T1, T2) using a psychosis add-on instrument based on the sections of psychotic symptoms in CIDI versions 1.0 and 2.0. The instrument consisted of 20 questions regarding lifetime PE. The 20 items included 15 delusional experiences and 5 hallucinatory experiences, described in detail elsewhere (van Nierop et al. 2012). Individuals with at least one lifetime PE were contacted for a re-interview by telephone. Re-interviews were conducted by an experienced clinician at the level of psychologist or psychiatrist within 8 weeks after the initial interview, using questions from the Structured Clinical Interview for DSM-IV. Findings from all re-interviews were discussed with a second clinician (van Nierop et al. 2012). PE were defined as clinically validated when the psychotic nature of the selfreported PE was confirmed at clinical re-interview. The response rates for the reinterviews at the three time points were 73\% (T0), 84\% (T1) and 81\% (T2), respectively. At baseline, lifetime PE were assessed. At both follow-up surveys, participants were asked about PE since the last interview. For the present analyses, only clinically validated PE were used. Individuals with self-reported PE who could not be reached for reinterview were excluded from the analyses. PE were defined present if the participant had at least one clinically validated PE. Incident PE was defined present if a participant reported at least one clinically validated PE at one of the follow-up surveys, but reported no PE at baseline.

\section{Childhood victimisation}

At baseline, childhood victimisation was assessed retrospectively. Participants were asked whether they had experienced emotional abuse, psychological abuse, physical 
abuse or sexual abuse before the age of 16 years. Consistent with previous analyses (ten Have et al. 2014), childhood abuse was defined present if a participant had experienced psychological abuse or emotional abuse on two or more occasions, or physical abuse / sexual abuse on one or more occasion. In addition, being bullied was assessed at baseline by asking participants if they had been bullied regularly before the age of 16 years. For the present study, childhood victimisation was defined present if a participant reported childhood abuse or bullying at baseline. Individuals with missing data on childhood victimisation were excluded from the present analyses $(n=140)$.

\section{Adult victimisation}

At baseline, participants were asked about lifetime violent and psychological victimisation by an intimate partner. In addition, lifetime sexual victimisation by any person in general since the age of 16 years was assessed. To increase the likelihood of these forms of victimisation being reported, the interviewer did not mention any type of victimisation during the interview. Instead, different forms of victimisation were listed and numbered in a booklet. Participants were asked to provide the numbers of the type of victimisation. Psychological victimisation included name-calling, offending, belittling, punishing unjustly, blackmailing and threatening. Physical victimisation included kicking, biting, hitting, trying to wound with an object (gun, knife, piece of wood, pair of scissors, other object) or hot water. Sexual victimisation included unwanted touching, forced undressing and forced sexual activity. Consistent with previous work (ten Have et al. 2014; Honings et al. 2016), psychological victimisation was defined present if it occurred on two or more occasions, and violent/sexual victimisation on one or more occasions.

At both follow-up measurements, participants were asked about violent, psychological and sexual victimisation since the last assessment by any person in general, and if so, by whom (i.e. partner, ex-partner, family member, acquaintance, stranger). To reach consistency with the baseline questions, physical victimisation and psychological victimisation at follow-up were defined present if the respective form of victimisation was perpetrated by an intimate partner. Sexual victimisation at follow-up was defined present if it was perpetrated by any person in general, conform baseline measurement. The frequencies for all victimisation outcomes were similar to the frequencies at baseline.

Incident physical victimisation by a partner (hereafter: physical victimisation), incident psychological victimisation by a partner (hereafter: psychological victimisation) and incident sexual victimisation (hereafter: sexual victimisation) were defined as present if the participant reported the respective type of victimisation at any of the follow-up interviews, while participants with the respective type of victimisation at baseline were excluded. In addition, a summary variable (any incident adult victimisation) was generated to identify participants who experienced any form of adult victimisation at followup, but had no victimisation at baseline. Participants without a partner at baseline and 
any of the follow-up measurements were excluded when analysing physical victimisation, psychological victimisation or the summary variable.

\section{Confounders}

Age, gender, low socio-economic status, past criminal activity and substance use disorders were hypothesized to be confounders in the present analyses (Morgan et al. 2016; de Mooij et al. 2015). Arrest was used as a measure of criminal activity. Self-reported arrest was obtained at baseline by asking participants if they had ever been arrested. In addition, CIDI 3.0 was used to define baseline, lifetime diagnoses of any substance use disorder. Finally, household income was a proxy for socio-economic status. The variable included three strata, based on monthly income: low (<€1500), middle (€1500-€3300) and high (>€3300).

\section{Statistical analyses}

All statistical analyses were performed using Stata version 13 (StataCorp. 2013). Baseline characteristics were assessed for the complete sample. In addition, subjects with clinically validated PE at baseline were compared with subjects without clinically validated PE, using chi-square tests and independent sample t-tests.

Logistic regression analyses were performed to answer the six research questions. All regression models included age, gender, household income (dummies of strata), baseline substance use disorders and arrest as covariates. The numbers of the analyses correspond with the numbers in Fig. 1.

1) Logistic regression analyses were performed to examine the association between baseline PE and incident adult victimisation at follow-up in the complete sample.

2) The association between baseline childhood victimisation and incident adult victimisation was assessed in a separate logistic regression analysis.

3) To assess whether childhood victimisation moderated the association between PE and adult victimisation, a logistic regression analysis was performed using incident adult victimisation as the dependent variable and $\mathrm{PE}$, childhood victimisation and the interaction term $\mathrm{PE}$ * childhood victimisation as the independent variables. In order to explore any interaction effect, a logistic regression analysis was performed using incident adult victimisation as the dependent variable and a categorical variable containing the following categories as the independent variable, modelled as dummies: (1) No PE, no childhood victimisation (reference group); (2) No PE, childhood victimisation present; (3) PE present, no childhood victimisation; (4) PE present, childhood victimisation present. If the interaction term was below alpha $(\alpha=0.10)$, the associations between PE, childhood victimisation and adult victimisation were analysed stratified by presence or absence of PE and childhood victimisation, respectively. 
4) The association between baseline adult victimisation and incident PE at follow-up was examined in a logistic regression analysis in the complete sample.

5) The association between childhood victimisation and incident PE was assessed in a logistic regression analysis in the complete sample.

6) The hypothesized moderating effect of childhood victimisation on the association between baseline adult victimisation and incident PE was examined by conducting a logistic regression analysis using incident PE as the dependent variable and adult victimisation, childhood victimisation and the interaction term adult victimisation * childhood victimisation as the independent variables. A logistic regression analysis was performed using incident PE as the dependent variable and a categorical variable containing the following categories as the independent variable: (1) No childhood victimisation, no adult victimisation (reference category); (2) childhood victimisation present, no adult victimisation; (3) no childhood victimisation, adult victimisation present; (4) childhood victimisation present, adult victimisation present. Again, if the $p$-value of the interaction term was below alpha $(\alpha=0.10)$, the associations between childhood victimisation, adult victimisation and PE were examined in stratified analyses.

\section{Results}

\section{Baseline characteristics}

At baseline, the complete sample included 6359 participants, after exclusion of individuals with self-reported PE who could not be reached for re-interview $(n=287)$. Of these participants, 5.3\% ( $n=340)$ reported clinically validated PE. More women than men reported PE (Table 1). Moreover, the proportion of individuals with PE differed significantly between the strata of household income. Subjects with PE were overrepresented in the low income group and underrepresented in the high-income group. Furthermore, the baseline prevalence of childhood victimisation, adult victimisation, lifetime substance use disorders and arrest was significantly higher in individuals with PE (Table 1). 


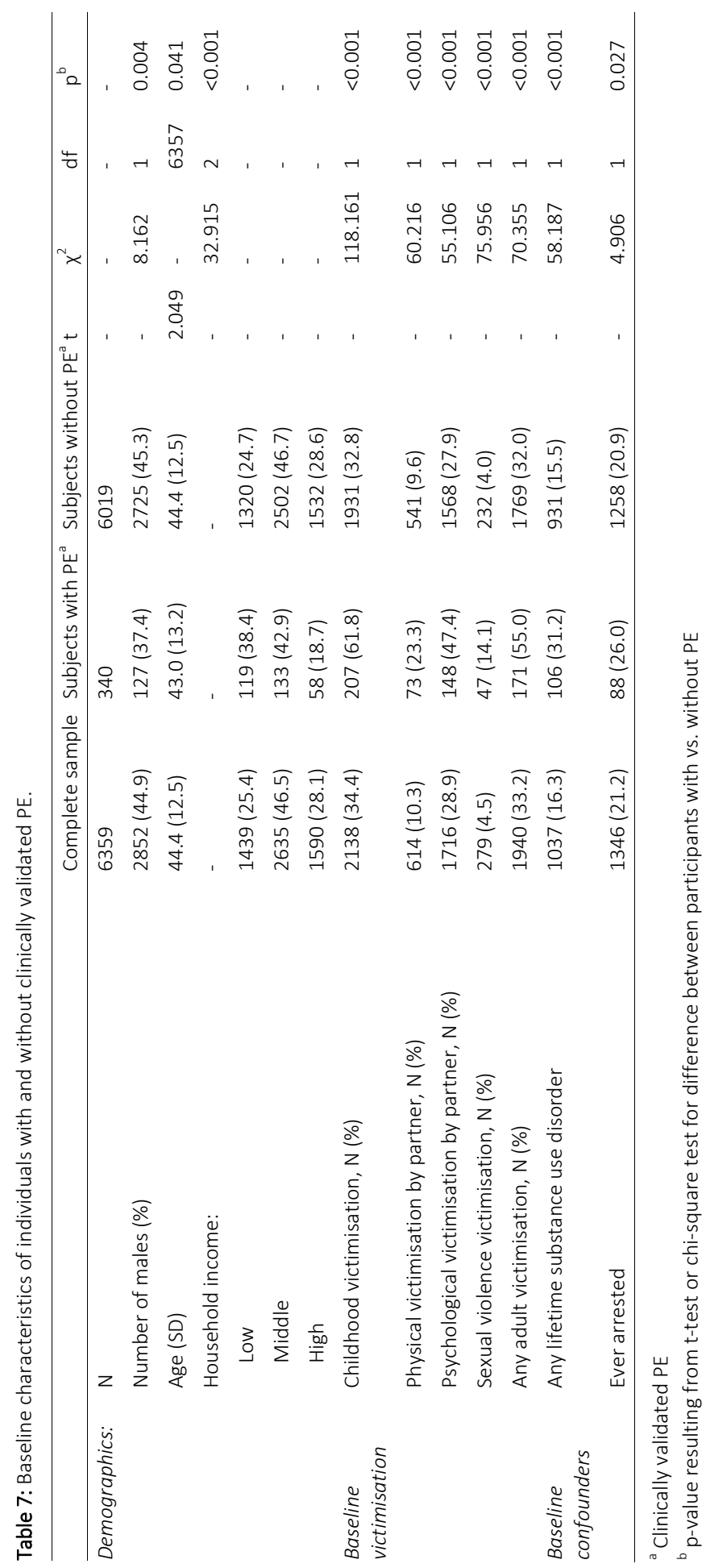


1. The association between baseline psychotic experiences and adult victimisation The odds ratio (OR) of the association between baseline PE and any incident adult victimisation was 2.09 (95\% Cl 0.79 - 5.56; Table 2). PE were associated with all forms of victimisation, but the OR was only statistically significant for the association between $P E$ and sexual victimisation (OR=3.51, 95\% Cl 1.54-7.96).

\section{The association between baseline childhood victimisation and adult victimisation}

Childhood victimisation was associated with all forms of adult victimisation, with ORs ranging from $2.70(95 \% \mathrm{Cl} 1.41$ - 5.16) for sexual victimisation to 5.49 (95\% Cl 2.26 13.34) for physical victimisation (Table 2 ).

\section{The interaction between psychotic experiences and childhood victimisation on the outcome adult victimisation}

The interaction term PE * Childhood victimisation was below alpha for the outcome any adult victimisation (Table 2; $\mathrm{p}=0.08$ ). Follow-up analysis of the interaction between childhood victimisation and PE on the outcome adult victimisation, using a categorical predictor, showed that isolated $\mathrm{PE}(\mathrm{OR}=4.49,95 \% \mathrm{Cl} 1.27-15.90)$ and isolated childhood victimisation $(\mathrm{OR}=3.73,95 \% \mathrm{Cl} 2.16-6.47)$ were associated with an increased risk of any adult victimisation. However, the co-occurrence of PE and childhood victimisation was associated with a lower risk of any adult victimisation than their isolated effects $(\mathrm{OR}=2.88,95 \% \mathrm{Cl} 0.62-13.39)$, thus indicating a negative interaction. Analyses in the subsample stratified by presence or absence of childhood victimisation showed that PE were associated with adult victimisation in the subsample without childhood victimisation (OR=4.81, 95\% Cl $1.34-17.29$ ). However, in the subsample with childhood victimisation there was no association between PE and adult victimisation. Similarly, childhood victimisation was associated with adult victimisation in the subsample without baseline PE $(O R=3.76,95 \% \mathrm{Cl} 2.17-6.53)$, but was not associated with adult victimisation in the subsample with baseline PE. Results for the outcomes physical victimisation and psychological victimisation were similar to the results of any adult victimisation and showed a trend towards a negative interaction as well ( $p=0.16$ for physical victimisation, $p=0.07$ for psychological victimisation). Interaction could not be examined in the model with sexual victimisation as the outcome, because none of the subjects had PE in absence of childhood victimisation. However, the co-occurrence of PE and childhood victimisation $(\mathrm{OR}=8.72,95 \% \mathrm{Cl} 3.40-22.32)$ showed a stronger association with sexual victimisation than isolated childhood victimisation ( $\mathrm{OR}=2.24,95 \% \mathrm{Cl} 1.10-4.57$ ). 


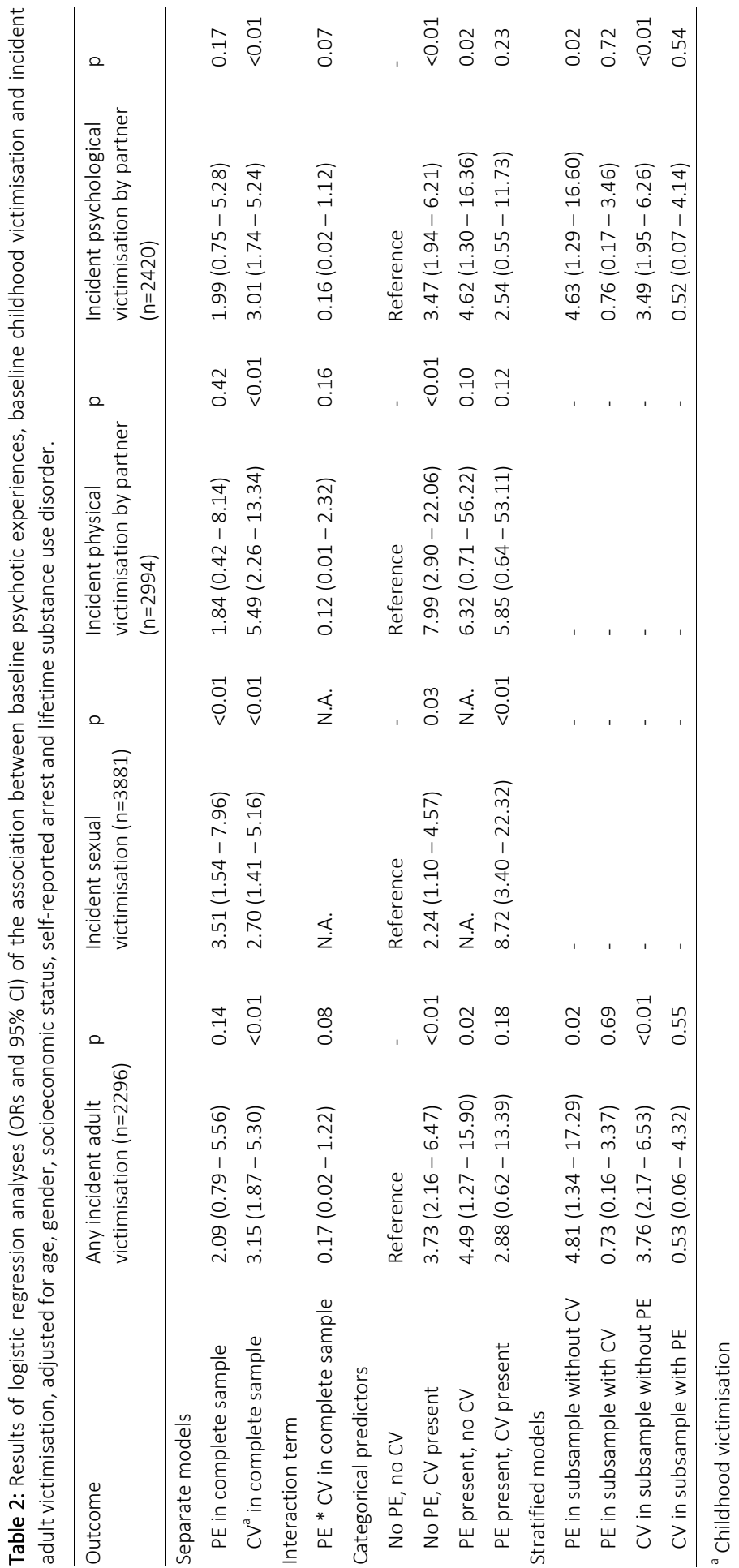


Chapter 5

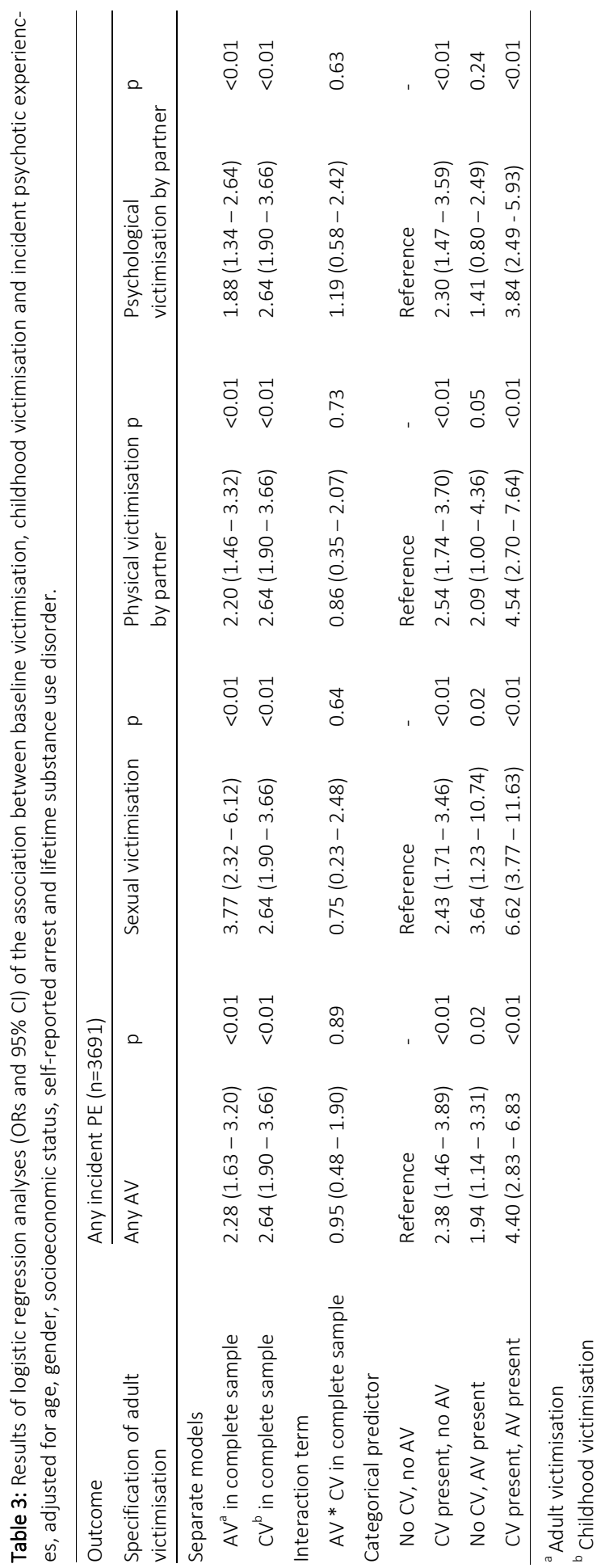


4. The association between baseline adult victimisation and incident psychotic experiences

In the complete sample, all forms of baseline adult victimisation were associated with incident PE, with ORs ranging from $1.88(95 \% \mathrm{Cl} 1.34-2.64)$ for psychological victimisation to $3.77(95 \% \mathrm{Cl} 2.32-6.12)$ for sexual victimisation, after adjustment for confounders (Table 3).

\section{The association between baseline childhood victimisation and incident psychotic} experiences

Childhood victimisation was associated with incident PE in the complete sample (Table 3; OR=2.64, 95\% Cl $1.90-3.66)$.

6. The interaction between childhood victimisation and adult victimisation on the outcome psychotic experiences

The risk of PE in subjects with both childhood victimisation and adult victimisation was not larger than the product of their risks in participants with isolated adult victimisation and participants with isolated childhood victimisation separately, indicating that there was no interaction between adult victimisation and childhood victimisation (Table 3).

\section{Discussion}

\section{Overview of results}

To our knowledge, the present study is the first to examine the bidirectional, longitudinal associations between PE and adult victimisation using a prospective, general population sample, while also assessing the moderating effect of childhood victimisation. It was hypothesized that PE increase the risk of incident adult victimisation, and that adult victimisation increases the risk of incident PE. Moreover, it was hypothesized that childhood victimisation increases the risk of both adult victimisation and PE and that the bidirectional associations between PE and adult victimisation would be increased by the presence of childhood victimisation (Fig. 1). The present results showed evidence for the hypothesized bidirectional association between adult victimisation and PE. However, the hypothesis of a positive interaction between childhood victimisation and both PE and adult victimisation was falsified (Fig. 2). 


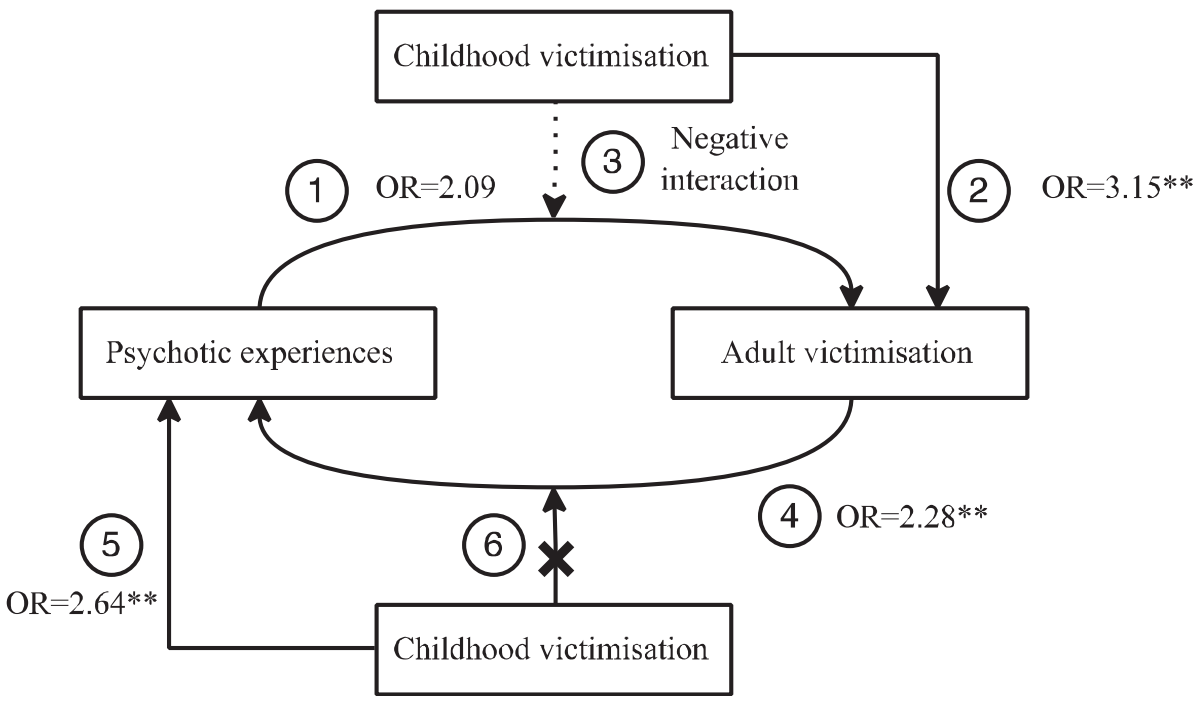

Figure. 2: Results of testing hypotheses on the bidirectional association between psychotic experiences and victimisation.

$* * p<0.01$

The association between baseline psychotic experiences and incident adult victimisation

Previous studies reported an association between psychotic disorder and adult victimisation (Morgan et al. 2016; Latalova et al. 2014; Kamperman et al. 2014; Choe et al. 2008). However, the temporal sequence of the association could not be determined in these studies because all studies used cross-sectional study designs. The present study showed that individuals with isolated PE or isolated childhood victimisation at baseline were at increased risk of any adult victimisation. However, contrary to our hypothesis that PE and childhood victimisation would act synergistically to increase the risk of adult victimisation, results showed that the co-occurrence of PE with childhood victimisation was associated with a lower risk of adult victimisation than isolated PE or isolated childhood victimisation. The results of the stratified analyses revealed that exposure to PE after childhood victimisation was not associated with adult victimisation, while PE increased the risk of adult victimisation in individuals without childhood victimisation. Similarly, exposure to childhood victimisation was not a risk factor for adult victimisation in adults exposed to PE, while childhood victimisation did increase the risk of adult victimisation in individuals not exposed to PE. Therefore, there was a negative interaction effect, pointing towards 'parallelism' instead of the hypothesized 'synergism' (Darroch 1997; Murray et al. 2002), thus suggesting that PE and childhood victimisation act through competing pathways in increasing risk for adult victimisation. In other 
words, any excess risk for adult victimisation would already have been consumed after isolated exposure to either PE or childhood victimisation.

The results for the outcomes physical victimisation and psychological victimisation were similar to the results of the outcome of any adult victimisation, showing a trend towards a negative interaction. However, interaction could not be examined for the outcome of sexual victimisation. As opposed to the results for physical victimisation and psychological victimisation, available results show that PE were associated with incident sexual victimisation in the subgroup with childhood victimisation. However, the interaction could not be analysed. Therefore, it is possible that the association between PE and adult sexual victimisation differs from the associations between PE and other forms of adult victimisation. This finding would be in line with previous research that found that childhood sexual abuse was differentially associated with psychosis compared to other victimising experiences during childhood (Bebbington et al. 2004; Read et al. 2005; Fry et al. 2012).

The mechanisms behind the association between PE and incident adult victimisation remain unclear. One possible explanation is that individuals with PE display disordered behaviour, for example arising from paranoid delusions, leading to social conflict and victimisation. Previous research showed that PE increase the risk of violence perpetration (Honings et al. 2016). Therefore, it is possible that adult victimisation occurs in response to violence perpetration. Another possible explanation is that individuals with $P E$ live in poorer social environments where adult victimisation is more likely to occur. However, the present analyses were adjusted for household income as a proxy for socio-economic status. Finally, it is possible that individuals with PE are more likely to report adult victimisation, for example because of paranoid interpretations of social interactions. To our knowledge, the reliability of adult victimisation reports in individuals with psychosis has not been studied. However, it has been shown that individuals with psychosis are able to provide reliable reports of childhood victimisation (Goodman et al. 1999; Fisher et al. 2011). Therefore, it is unlikely that the association between PE and adult victimisation can be fully attributed to differential reporting of adult victimisation by individuals with PE.

\section{The association between baseline adult victimisation and incident psychotic experiences}

Results of the present study showed that all forms of adult victimisation were associated with the development of incident PE. This confirms our hypothesis that the association between PE and adult victimisation is bidirectional, similar to the previously reported bidirectional association between PE and childhood victimisation (Kelleher et al. 2013). Consistent with previous work (Morgan and Gayer-Anderson 2016; Lataster et al. 2006; Varese et al. 2012; Read et al. 2005; Kelleher et al. 2013), childhood victimisation was associated with incident PE, both in the presence and absence of co-occurring adult 
victimisation. However, contrary to our hypothesis of a positive interaction between childhood victimisation and adult victimisation for the outcome of incident PE, the risk of $P E$ in subjects with both childhood victimisation and adult victimisation was not larger than the product of the isolated risks. This finding is not fully consistent with the previous literature, which showed positive interactions between childhood victimisation and adult victimisation for the outcome PE (Bebbington et al. 2011; Lataster et al. 2012; Morgan et al. 2014b). However, all studies used a wide variety of definitions of childhood victimisation and adult victimisation, thus impeding direct comparison. The present results show that childhood victimisation and adult victimisation are independent, cumulative risk factors for PE. This finding is relevant, since poly-victimisation is prevalent among individuals with severe mental illness (Kamperman et al. 2014; Bebbington et al. 2004). Moreover, this finding shows that there are two pathways from childhood victimisation to psychosis: one direct one and one indirect one through adult victimisation.

Literature on the mechanisms behind the association between adult victimisation and incident psychosis is scarce. However, both adult victimisation and childhood victimisation have been linked to various non-psychotic mental disorders (Lagdon et al. 2014; Burnam et al. 1988; Mueser et al. 2002; Logan 2006; Kessler et al. 2010). Since PE are prevalent among individuals with non-psychotic mental disorders (Kelleher et al. 2012; van Os et al. 2000) and have been identified as an indicator of severity in nonpsychotic psychopathology (Wigman et al. 2012; Wigman et al. 2014; Perlis et al. 2011; Guloksuz et al. 2015; Kelleher et al. 2012), the link between adult victimisation and incident PE is possibly confounded by the presence of non-psychotic psychopathology (Wigman et al. 2012; Wigman et al. 2014; Perlis et al. 2011; Guloksuz et al. 2015; Kelleher et al. 2012). Furthermore, it is likely that the mechanisms underlying the impact of adult victimisation on psychosis are similar to the mechanisms mediating impact of childhood victimisation on psychosis (Galletly et al. 2011; Lataster et al. 2012). Various biological and psychological processes have been associated with both psychosis and childhood victimisation (Ruby et al. 2014; Morgan and Gayer-Anderson 2016; Freeman and Garety 2014). Biological processes that link victimisation with an increase in psychosis risk include hyperactivation and sensitization of the hypothalamic-pituitaryadrenal axis, decreased hippocampal volume, reduced brain-derived neurotrophic factor (Ruby et al. 2014) and increased dopamine release (Morgan and Gayer-Anderson 2016). Furthermore, victimisation may increase the risk of psychosis psychologically by contributing to the development of a worrying thinking style, negative beliefs about the self and reasoning biases such as jumping to conclusions (Freeman and Garety 2014). However, more research to examine the mechanisms underlying the link between psychosis and adult victimisation is needed. 


\section{Strengths and limitations}

Strength of the present study is the prospective, longitudinal study design that enables the bidirectional assessment of psychosis and victimisation in a general population sample, while controlling for various confounders identified in the literature. Another strength is the use of clinically validated PE instead of self-reported PE (Linscott and van Os 2013).

The results of the study should be interpreted in the light of some limitations. First, the definitions of incident physical and psychological victimisation had to be restricted to victimisation by an intimate partner in order to remain consistent with the baseline definitions in the dataset. To overcome this limitation, individuals without a partner at baseline and any of the follow-up measurements were excluded from the analyses when using physical victimisation, psychological victimisation or any adult victimisation as the outcome, resulting in an exclusion of 1066 individuals for the present analysis. To our knowledge, no previous study examined whether victimisation by an intimate partner may be differently associated with psychosis than victimisation by any person. In order to examine this, sensitivity analyses were conducted examining the association between baseline PE and adult victimisation by any person at follow-up, while excluding individuals without a partner at baseline. The results of these sensitivity analyses were similar to the results of the main analyses in terms of effect size. However, statistical significance was greater as a result of increased statistical power. Therefore, the association between PE and adult victimisation perpetrated by any person appears to be similar to the association between PE and adult victimisation perpetrated by an intimate partner. However, more research is required to investigate this issue.

Second, baseline data on household income was missing for 604 individuals. Again, sensitivity analyses were conducted using the missing data as a separate category. Results of these analyses were similar to the main results.

Third, statistical power was low in some analyses, in particular in the interaction analyses. Thus, in order to screen for potential interactions, alpha for interaction effects was set at 0.10 . Raising the alpha to 0.10 increases the risk of false positive results. Therefore, it is possible that some results represent type I error. More studies are required to replicate the interactions identified in this study.

Furthermore, data about baseline victimisation was collected retrospectively. Since participants in this study were aged 18-64 years, it is possible that differences in recall impacted the results. Previous studies showed evidence for age-related differential recall, with individuals underreporting victimisation as age increases (Yoshihama and Gillespie 2002; Widom and Morris 1997; Widom and Shepard 1996). Therefore, it is possible that the associations between victimisation and psychosis identified in this study would be stronger in the absence of age-related differential recall. In addition, the risk of exposure to adult victimisation and PE varies by age, suggesting an interaction effect between age, PE and adult victimisation. To examine this, sensitivity analyses 
were conducted testing for interaction effects between the various predictor variables and five age categories. Results of this analysis showed that there was no evidence for interaction between any of the predictor variables and age when studying incident PE. In addition, the association between baseline PE and incident AV did not differ between the various age groups above 25 years, but there was some evidence for a decreased association in the youngest age group (18-25 years). However, statistical power in the youngest age group was insufficient to further explore this finding. Other studies with more young adults are needed to study this hypothesis.

Finally, follow-up data was missing for 2028 participants (attrition=30.5\%). Attrition was associated with sociodemographic factors, but not with mental health status, making it unlikely that attrition would induce bias (de Graaf et al. 2013). In addition, attrition was not associated with baseline PE or baseline victimisation status. Therefore, selective attrition is unlikely to have biased the results.

\section{Conclusions and implications}

The present study did not find evidence for all hypotheses (Fig. 2). In order to integrate this updated evidence, Fig. 2 was transformed into Fig. 3, removing the connections that were not supported by the present results. PE and childhood victimisation were identified as competing risk factors for adult victimisation. In addition, childhood victimisation and adult victimisation were independent, cumulative risk factors for PE. Thus, PE and adult victimisation are bidirectionally associated in individuals without childhood victimisation. In individuals with childhood victimisation, there are two pathways from childhood victimisation to PE: one direct and one indirect through adult victimisation. In conclusion, psychosis and victimisation are strongly interconnected throughout the life course, resulting in a complex interplay in which childhood victimisation and adult victimisation lead to both PE and re-victimising experiences. Because victimisation across the life course has been associated with several adverse consequences (Palm et al. 2016; Hodgins et al. 2009; Lysaker et al. 2001; Newman et al. 2010; Kessler et al. 2010), prevention strategies against victimisation, both during childhood and during adulthood, are important to prevent individuals from entering a spiral leading to mental illness and re-victimisation. Moreover, intervention programs are needed to prevent further re-victimisation in individuals who have already experienced victimisation. However, the development of adequate prevention and intervention programs requires further understanding of the mechanisms underlying the associations identified in the present study. Therefore, more longitudinal research is required to obtain a deeper understanding of the complex interplay between psychosis and victimisation across the life course. 


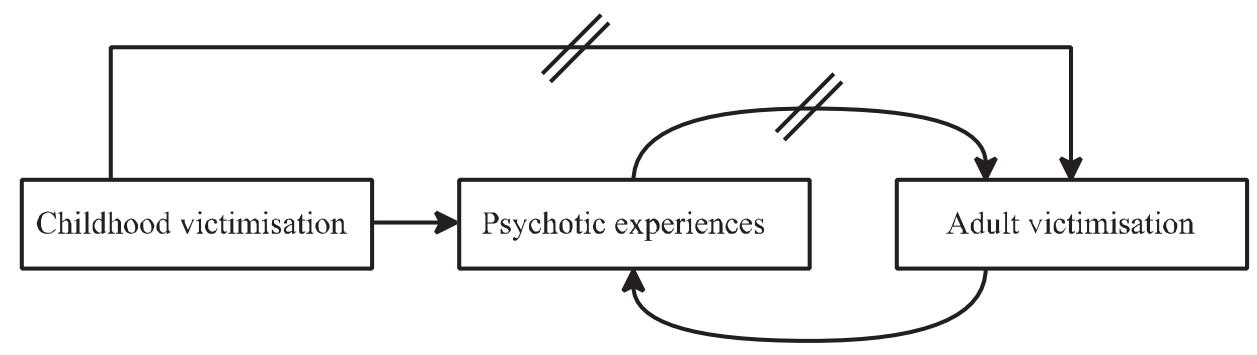

Figure. 3: The complex interplay of psychosis and victimisation across the life course.

// points towards parallelism, i.e. childhood victimisation and psychotic experiences are competing risk factors for adult victimisation.

\section{Acknowledgements}

NEMESIS-2 is conducted by the Netherlands Institute of Mental Health and Addiction (Trimbos Institute) in Utrecht. Financial support has been received from the Ministry of Health, Welfare and Sport, with supplementary support from the Netherlands Organization for Health Research and Development (ZonMw) and the Genetic Risk and Outcome of Psychosis (GROUP) investigators. 


\section{References}

Barrios, Y. V., Gelaye, B., Zhong, Q., Nicolaidis, C., Rondon, M. B., Garcia, P. J., Sanchez, P. A., Sanchez, S. E. \& Williams, M. A. 2015. Association of childhood physical and sexual abuse with intimate partner violence, poor general health and depressive symptoms among pregnant women. PLoS One, 10, e0116609.

Bebbington, P., Jonas, S., Kuipers, E., King, M., Cooper, C., Brugha, T., Meltzer, H., Mcmanus, S. \& Jenkins, R. 2011. Childhood sexual abuse and psychosis: data from a cross-sectional national psychiatric survey in England. Br J Psychiatry, 199, 29-37.

Bebbington, P. E., Bhugra, D., Brugha, T., Singleton, N., Farrell, M., Jenkins, R., Lewis, G. \& Meltzer, H. 2004. Psychosis, victimisation and childhood disadvantage: evidence from the second British National Survey of Psychiatric Morbidity. Br J Psychiatry, 185, 220-6.

Bensley, L., Van Eenwyk, J. \& Wynkoop Simmons, K. 2003. Childhood family violence history and women's risk for intimate partner violence and poor health. Am J Prev Med, 25, 38-44.

Blom, H., Hogberg, U., Olofsson, N. \& Danielsson, I. 2014. Strong association between earlier abuse and revictimization in youth. BMC Public Health, 14, 715 .

Burnam, M. A., Stein, J. A., Golding, J. M., Siegel, J. M., Sorenson, S. B., Forsythe, A. B. \& Telles, C. A. 1988. Sexual assault and mental disorders in a community population. J Consult Clin Psychol, 56, 843-50.

Choe, J. Y., Teplin, L. A. \& Abram, K. M. 2008. Perpetration of violence, violent victimization, and severe mental illness: balancing public health concerns. Psychiatr Serv, 59, 153-64.

Coid, J., Petruckevitch, A., Feder, G., Chung, W., Richardson, J. \& Moorey, S. 2001. Relation between childhood sexual and physical abuse and risk of revictimisation in women: a cross-sectional survey. Lancet, 358, 450-4.

Coid, J. W., Ullrich, S., Bebbington, P., Fazel, S. \& Keers, R. 2016. Paranoid Ideation and Violence: Metaanalysis of Individual Subject Data of 7 Population Surveys. Schizophr Bull, 42, 907-15.

Crisp, A. H., Gelder, M. G., Rix, S., Meltzer, H. I. \& Rowlands, O. J. 2000. Stigmatisation of people with mental illnesses. Br J Psychiatry, 177, 4-7.

Darroch, J. 1997. Biologic synergism and parallelism. Am J Epidemiol, 145, 661-8.

De Graaf, R., Ten Have, M. \& Van Dorsselaer, S. 2010. The Netherlands Mental Health Survey and Incidence Study-2 (NEMESIS-2): design and methods. Int J Methods Psychiatr Res, 19, 125-41.

De Graaf, R., Van Dorsselaer, S., Tuithof, M. \& Ten Have, M. 2013. Sociodemographic and psychiatric predictors of attrition in a prospective psychiatric epidemiological study among the general population. Result of the Netherlands Mental Health Survey and Incidence Study-2. Compr Psychiatry, 54, 1131-9.

De Mooij, L. D., Kikkert, M., Lommerse, N. M., Peen, J., Meijwaard, S. C., Theunissen, J., Duurkoop, P. W., Goudriaan, A. E., Van, H. L., Beekman, A. T. \& Dekker, J. J. 2015. Victimisation in adults with severe mental illness: prevalence and risk factors. Br J Psychiatry, 207, 515-22.

Douglas, K. S., Guy, L. S. \& Hart, S. D. 2009. Psychosis as a risk factor for violence to others: a meta-analysis. Psychol Bull, 135, 679-706.

Fazel, S., Gulati, G., Linsell, L., Geddes, J. R. \& Grann, M. 2009. Schizophrenia and violence: systematic review and meta-analysis. PLoS Med, 6, e1000120.

Fazel, S. \& Yu, R. 2011. Psychotic disorders and repeat offending: systematic review and meta-analysis. Schizophr Bull, 37, 800-10.

Fisher, H. L., Craig, T. K., Fearon, P., Morgan, K., Dazzan, P., Lappin, J., Hutchinson, G., Doody, G. A., Jones, P. B., Mcguffin, P., Murray, R. M., Leff, J. \& Morgan, C. 2011. Reliability and comparability of psychosis patients' retrospective reports of childhood abuse. Schizophr Bull, 37, 546-53.

Freeman, D. \& Garety, P. 2014. Advances in understanding and treating persecutory delusions: a review. Soc Psychiatry Psychiatr Epidemiol, 49, 1179-89.

Fry, D., Mccoy, A. \& Swales, D. 2012. The consequences of maltreatment on children's lives: a systematic review of data from the East Asia and Pacific Region. Trauma Violence Abuse, 13, 209-33.

Galletly, C., Van Hooff, M. \& Mcfarlane, A. 2011. Psychotic symptoms in young adults exposed to childhood trauma--a 20 year follow-up study. Schizophr Res, 127, 76-82. 
Goodman, L. A., Thompson, K. M., Weinfurt, K., Corl, S., Acker, P., Mueser, K. T. \& Rosenberg, S. D. 1999. Reliability of reports of violent victimization and posttraumatic stress disorder among men and women with serious mental illness. J Trauma Stress, 12, 587-99.

Guloksuz, S., Van Nierop, M., Lieb, R., Van Winkel, R., Wittchen, H. U. \& Van Os, J. 2015. Evidence that the presence of psychosis in non-psychotic disorder is environment-dependent and mediated by severity of non-psychotic psychopathology. Psychol Med, 45, 2389-401.

Haro, J. M., Arbabzadeh-Bouchez, S., Brugha, T. S., De Girolamo, G., Guyer, M. E., Jin, R., Lepine, J. P., Mazzi, F., Reneses, B., Vilagut, G., Sampson, N. A. \& Kessler, R. C. 2006. Concordance of the Composite International Diagnostic Interview Version 3.0 (CIDI 3.0) with standardized clinical assessments in the WHO World Mental Health surveys. Int J Methods Psychiatr Res, 15, 167-80.

Hart, C., De Vet, R., Moran, P., Hatch, S. L. \& Dean, K. 2012. A UK population-based study of the relationship between mental disorder and victimisation. Soc Psychiatry Psychiatr Epidemiol, 47, 1581-90.

Hodgins, S., Lincoln, T. \& Mak, T. 2009. Experiences of victimisation and depression are associated with community functioning among men with schizophrenia. Soc Psychiatry Psychiatr Epidemiol, 44, 448-57.

Honings, S., Drukker, M., Ten Have, M., De Graaf, R., Van Dorsselaer, S. \& Van Os, J. 2016. Psychotic Experiences and Risk of Violence Perpetration and Arrest in the General Population: A Prospective Study. PLoS One, 11, e0159023.

Kamperman, A. M., Henrichs, J., Bogaerts, S., Lesaffre, E. M., Wierdsma, A. I., Ghauharali, R. R., Swildens, W., Nijssen, Y., Van Der Gaag, M., Theunissen, J. R., Delespaul, P. A., Van Weeghel, J., Van Busschbach, J. T., Kroon, H., Teplin, L. A., Van De Mheen, D. \& Mulder, C. L. 2014. Criminal victimisation in people with severe mental illness: a multi-site prevalence and incidence survey in the Netherlands. PLoS One, 9, e91029.

Kelleher, I., Keeley, H., Corcoran, P., Lynch, F., Fitzpatrick, C., Devlin, N., Molloy, C., Roddy, S., Clarke, M. C., Harley, M., Arseneault, L., Wasserman, C., Carli, V., Sarchiapone, M., Hoven, C., Wasserman, D. \& Cannon, M. 2012. Clinicopathological significance of psychotic experiences in non-psychotic young people: evidence from four population-based studies. Br J Psychiatry, 201, 26-32.

Kelleher, I., Keeley, H., Corcoran, P., Ramsay, H., Wasserman, C., Carli, V., Sarchiapone, M., Hoven, C., Wasserman, D. \& Cannon, M. 2013. Childhood trauma and psychosis in a prospective cohort study: cause, effect, and directionality. Am J Psychiatry, 170, 734-41.

Kessler, R. C., Mclaughlin, K. A., Green, J. G., Gruber, M. J., Sampson, N. A., Zaslavsky, A. M., Aguilar-Gaxiola, S., Alhamzawi, A. O., Alonso, J., Angermeyer, M., Benjet, C., Bromet, E., Chatterji, S., De Girolamo, G., Demyttenaere, K., Fayyad, J., Florescu, S., Gal, G., Gureje, O., Haro, J. M., Hu, C. Y., Karam, E. G., Kawakami, N., Lee, S., Lepine, J. P., Ormel, J., Posada-Villa, J., Sagar, R., Tsang, A., Ustun, T. B., Vassilev, S., Viana, M. C. \& Williams, D. R. 2010. Childhood adversities and adult psychopathology in the WHO World Mental Health Surveys. Br J Psychiatry, 197, 378-85.

Khalifeh, H., Oram, S., Osborn, D., Howard, L. M. \& Johnson, S. 2016. Recent physical and sexual violence against adults with severe mental illness: a systematic review and meta-analysis. Int Rev Psychiatry, 28, 433-451.

Lagdon, S., Armour, C. \& Stringer, M. 2014. Adult experience of mental health outcomes as a result of intimate partner violence victimisation: a systematic review. Eur J Psychotraumatol, 5.

Large, M. M. \& Nielssen, O. 2011. Violence in first-episode psychosis: a systematic review and meta-analysis. Schizophr Res, 125, 209-20.

Latalova, K., Kamaradova, D. \& Prasko, J. 2014. Violent victimization of adult patients with severe mental illness: a systematic review. Neuropsychiatr Dis Treat, 10, 1925-39.

Lataster, J., Myin-Germeys, I., Lieb, R., Wittchen, H. U. \& Van Os, J. 2012. Adversity and psychosis: a 10-year prospective study investigating synergism between early and recent adversity in psychosis. Acta Psychiatr Scand, 125, 388-99.

Lataster, T., Van Os, J., Drukker, M., Henquet, C., Feron, F., Gunther, N. \& Myin-Germeys, I. 2006. Childhood victimisation and developmental expression of non-clinical delusional ideation and hallucinatory experiences: victimisation and non-clinical psychotic experiences. Soc Psychiatry Psychiatr Epidemiol, 41, 423-8. 


\section{Chapter 5}

Linscott, R. J. \& Van Os, J. 2013. An updated and conservative systematic review and meta-analysis of epidemiological evidence on psychotic experiences in children and adults: on the pathway from proneness to persistence to dimensional expression across mental disorders. Psychol Med, 43, 1133-49.

Logan, T. 2006. Women and Victimization: Contributing Factors, Interventions, and Implications, American Psychological Association.

Lysaker, P. H., Meyer, P. S., Evans, J. D., Clements, C. A. \& Marks, K. A. 2001. Childhood sexual trauma and psychosocial functioning in adults with schizophrenia. Psychiatr Serv, 52, 1485-8.

Mcintyre, J. K. \& Widom, C. S. 2011. Childhood victimization and crime victimization. J Interpers Violence, 26, 640-63.

Morgan, C., Reininghaus, U., Fearon, P., Hutchinson, G., Morgan, K., Dazzan, P., Boydell, J., Kirkbride, J. B., Doody, G. A., Jones, P. B., Murray, R. M. \& Craig, T. 2014a. Modelling the interplay between childhood and adult adversity in pathways to psychosis: initial evidence from the AESOP study. Psychol Med, 44, 407-19.

Morgan, C., Reininghaus, U., Reichenberg, A., Frissa, S., Team, S. E. S., Hotopf, M. \& Hatch, S. L. 2014b. Adversity, cannabis use and psychotic experiences: evidence of cumulative and synergistic effects. Br J Psychiatry, 204, 346-53.

Morgan, C. \& Gayer-Anderson, C. 2016. Childhood adversities and psychosis: evidence, challenges, implications. World Psychiatry, 15, 93-102.

Morgan, V. A., Morgan, F., Galletly, C., Valuri, G., Shah, S. \& Jablensky, A. 2016. Sociodemographic, clinical and childhood correlates of adult violent victimisation in a large, national survey sample of people with psychotic disorders. Soc Psychiatry Psychiatr Epidemiol, 51, 269-79.

Mueser, K. T., Rosenberg, S. D., Goodman, L. A. \& Trumbetta, S. L. 2002. Trauma, PTSD, and the course of severe mental illness: an interactive model. Schizophr Res, 53, 123-43.

Murray, R. M., Jones, P. B., Susser, E., Van Os, J. \& Cannon, M. 2002. The Epidemiology of Schizophrenia, Cambridge University Press.

Newman, J. M., Turnbull, A., Berman, B. A., Rodrigues, S. \& Serper, M. R. 2010. Impact of traumatic and violent victimization experiences in individuals with schizophrenia and schizoaffective disorder. $J$ Nerv Ment Dis, 198, 708-14.

Palm, A., Danielsson, I., Skalkidou, A., Olofsson, N. \& Hogberg, U. 2016. Violence victimisation-a watershed for young women's mental and physical health. Eur J Public Health, 26, 861-867.

Perlis, R. H., Uher, R., Ostacher, M., Goldberg, J. F., Trivedi, M. H., Rush, A. J. \& Fava, M. 2011. Association between bipolar spectrum features and treatment outcomes in outpatients with major depressive disorder. Arch Gen Psychiatry, 68, 351-60.

Read, J., Van Os, J., Morrison, A. P. \& Ross, C. A. 2005. Childhood trauma, psychosis and schizophrenia: a literature review with theoretical and clinical implications. Acta Psychiatr Scand, 112, 330-50.

Ruby, E., Polito, S., Mcmahon, K., Gorovitz, M., Corcoran, C. \& Malaspina, D. 2014. Pathways Associating Childhood Trauma to the Neurobiology of Schizophrenia. Front Psychol Behav Sci, 3, 1-17.

Statacorp. 2013. Stata Statistical Software: Release 13. College Station, TX: StataCorp LP.

Ten Have, M., De Graaf, R., Van Weeghel, J. \& Van Dorsselaer, S. 2014. The association between common mental disorders and violence: to what extent is it influenced by prior victimization, negative life events and low levels of social support? Psychol Med, 44, 1485-98.

Van Nierop, M., Van Os, J., Gunther, N., Myin-Germeys, I., De Graaf, R., Ten Have, M., Van Dorsselaer, S., Bak, M. \& Van Winkel, R. 2012. Phenotypically continuous with clinical psychosis, discontinuous in need for care: evidence for an extended psychosis phenotype. Schizophr Bull, 38, 231-8.

Van Os, J., Hanssen, M., Bijl, R. \& Ravelli, A. 2000. Straus (1969) revisited: A psychosis continuum in the general population? Schizophrenia Research, 45, 11-20.

Van Os, J. \& Linscott, R. J. 2012. Introduction: The extended psychosis phenotype--relationship with schizophrenia and with ultrahigh risk status for psychosis. Schizophr Bull, 38, 227-30.

Van Os, J. \& Reininghaus, U. 2016. Psychosis as a transdiagnostic and extended phenotype in the general population. World Psychiatry, 15, 118-24. 
Varese, F., Smeets, F., Drukker, M., Lieverse, R., Lataster, T., Viechtbauer, W., Read, J., Van Os, J. \& Bentall, R. P. 2012. Childhood adversities increase the risk of psychosis: a meta-analysis of patient-control, prospective- and cross-sectional cohort studies. Schizophr Bull, 38, 661-71.

Widom, C. S. \& Shepard, R. L. 1996. Accuracy of adult recollections of childhood victimization: Part 1. Childhood physical abuse. Psychological Assessment, 8, 412-421.

Widom, C. S. \& Morris, S. 1997. Accuracy of adult recollections of childhood victimization, Part 2: Childhood sexual abuse. Psychological assessment, 9, 34.

Widom, C. S., Czaja, S. J. \& Dutton, M. A. 2008. Childhood victimization and lifetime revictimization. Child Abuse Negl, 32, 785-96.

Wigman, J. T., Van Nierop, M., Vollebergh, W. A., Lieb, R., Beesdo-Baum, K., Wittchen, H. U. \& Van Os, J. 2012. Evidence that psychotic symptoms are prevalent in disorders of anxiety and depression, impacting on illness onset, risk, and severity--implications for diagnosis and ultra-high risk research. Schizophr Bull, 38, 247-57.

Wigman, J. T., Van Os, J., Abidi, L., Huibers, M. J., Roelofs, J., Arntz, A., Kelleher, I. \& Peeters, F. P. 2014. Subclinical psychotic experiences and bipolar spectrum features in depression: association with outcome of psychotherapy. Psychol Med, 44, 325-36.

Wolke, D., Lereya, S. T., Fisher, H. L., Lewis, G. \& Zammit, S. 2014. Bullying in elementary school and psychotic experiences at 18 years: a longitudinal, population-based cohort study. Psychol Med, 44, 2199-211.

Yoshihama, M. \& Gillespie, B. W. 2002. Age adjustment and recall bias in the analysis of domestic violence data: Methodological improvements through the application of survival analysis methods. Journal of Family Violence, 17, 199-221. 

Chapter

Discussion 

The central themes of this thesis were psychosis and multidirectional violence. Although many studies to date examined the link between psychotic disorders and violence, less is known about the link between psychotic experiences and violence. Therefore, using three prospective, general population samples, the associations between psychotic experiences on the one hand and self-directed violence, interpersonal violence perpetration and interpersonal violence victimisation on the other, were examined in this thesis. In this chapter, the studies presented in the previous chapters will be summarized and discussed in a broader context.

\section{Psychotic experiences and self-directed violence}

In chapters 2 and 3, the associations between psychotic experiences and self-directed violence were examined. Although numerous studies on the association between psychotic experiences and self-directed violence were published in recent years, results were often contradictory (Martin et al. 2015; Sharifi et al. 2015; Kelleher et al. 2013a). Therefore, a systematic review and meta-analysis was performed in order to summarize and integrate the evidence. More specifically, the associations between psychotic experiences and non-suicidal self-injury, suicidal ideation and suicidal behaviour were examined in general population studies.

Results of the main meta-analysis showed a strong link between psychotic experiences and any self-directed violence (Honings et al. 2016a). Studies on the association between psychotic experiences and non-suicidal self-injurious behaviour were scarce, but a meta-analysis including all three available studies showed a statistically significant association. Similarly, psychotic experiences were associated with both suicidal ideation and suicidal behaviour, and the pooled odds ratios increased as the severity of the outcome measure increased. Sensitivity analyses and metaregression analyses were conducted in order to examine the influence of study design and adjustment for confounders. Although most included studies used a cross-sectional study design, a sensitivity analysis including only longitudinal studies confirmed the results of the main metaanalysis. In addition, metaregression analysis showed that reported odds ratios in crosssectional studies were not significantly smaller than reported odds ratios in longitudinal study. However, results of the metaregression analyses examining the influence of adjustment for depression, showed that reported adjusted odds ratios were significantly smaller than unadjusted odds ratios. A sensitivity analysis of the adjusted, longitudinal studies confirmed this finding, showing that the excess risk of self-directed violence in individuals with psychotic experiences reduced $74 \%$ after adjustment for depression and other mental disorders. Thus, there was strong evidence that the association between psychotic experiences and self-directed violence was confounded by the presence of co-occurring non-psychotic psychopathology, which is highly prevalent among individuals with psychotic experiences (Jeppesen et al. 2015; van Os et al. 2000; 
DeVylder et al. 2014; Kelleher et al. 2012; van Nierop et al. 2012; Wigman et al. 2012; Johns et al. 2004; Bevan Jones et al. 2012; Fisher et al. 2013; Calkins et al. 2014). Moreover, there was considerable scope for residual confounding in the studies, since most studies adjusted their analyses at the level of the dichotomous, clinical phenotype.

In conclusion, the systematic review and meta-analysis showed an association between psychotic experiences and self-directed violence. However, it remained unclear whether there was a specific association, or whether the reported association reflected the increased risk of self-directed violence in individuals in mental distress, caused by co-occurring non-psychotic psychopathology. Therefore, more longitudinal research was required to answer this question.

In chapter 3 , the results of a large, longitudinal study on the association between psychotic experiences and suicidal ideation and suicidal behaviour were presented (Honings et al. 2016b). It was attempted to disentangle the association between psychotic experiences and suicidal ideation and suicidal behaviour from connected nonpsychotic psychopathology. For this study, three prospective, general population cohorts were merged, resulting in a sample of 15.837 individuals. In accordance with the results of the meta-analysis, it was hypothesized that the previously reported association between psychotic experiences and suicidal ideation and suicidal behaviour, reflects the increased risk of these outcomes in individuals with non-psychotic mental disorders or multidimensional psychopathology under the threshold of a diagnosable mental disorder. In order to test this hypothesis, the association between psychotic experiences and suicidal ideation and suicidal behaviour was tested in both the complete sample and in subsamples stratified by presence or absence of mental disorders, while also adjusting for dimensional psychopathology in order to minimize residual confounding. Results showed that in the complete sample, psychotic experiences increased the risk of both suicidal ideation and suicidal behaviour. However, adjustment for dimensional psychopathology (i.e. depression, mania and anxiety symptoms), decreased the odds ratios and rendered the association between psychotic experiences and suicidal ideation statistically non-significant (Honings et al. 2016c). Consistent with results from the meta-analysis (chapter 2 ), the excess risk of suicidal ideation decreased by $70 \%$ after adjustment for non-psychotic psychopathology. Analyses in the subgroups stratified by mental disorder showed that in the subgroup without mental disorder, psychotic experiences were not associated with suicidal ideation or suicidal behaviour after adjustment for dimensional non-psychotic psychopathology. Similarly, in the subgroup with mental disorder, psychotic experiences were not associated with suicidal ideation after adjustment for non-psychotic psychopathology. However, psychotic experiences were strongly associated with suicide attempts in the subgroup with mental disorder, even in the fully adjusted models.

The results presented in chapters 2 and 3 confirmed the hypothesis that the association between psychotic experiences and suicidal ideation and suicidal behaviour is nonspecific, but reflects the increased risk of these outcomes in individuals with non- 
psychotic mental disorders or non-psychotic psychopathology under the threshold of a mental disorder. This finding is consistent with the study of Jahn et al. (2016), who reported that the relation between schizotypy and suicidal ideation could be explained by depressive symptoms and low self-esteem. On the other hand, the results presented in chapter 2 and 3 were not consistent with the results of the study by Schimanski et al. (2017), who found that the association between schizophrenia liability and suicidal ideation could not be explained by depressed affect. However, the sample used in this study was small ( $n=387)$.

Previously, various studies showed that the occurrence of psychotic experiences in individuals with non-psychotic pathology, for instance mood disorders and anxiety disorders, was associated with various adverse outcomes, including increased symptom severity, poorer illness course, increased service use and poor treatment outcome (Wigman et al. 2012; Wigman et al. 2014; Kelleher et al. 2012). These results led to the suggestion that psychotic experiences operate as an indicator of illness severity in individuals with non-psychotic psychopathology (Wigman et al. 2014). The results of chapter 3 confirm this hypothesis, as psychotic experiences were strongly associated with suicide attempts in individuals with non-psychotic mental disorders.

Several questions remain unanswered after interpreting the results presented in chapters 2 and 3. First, it remains unclear how psychotic experiences and non-psychotic symptoms interact on the pathway to self-directed violence. Research has shown that psychotic and non-psychotic symptoms do not vary in isolation, but make an impact on each other over time (Borsboom and Cramer 2013; van Os 2013). This has led to the suggestion of a transdiagnostic network model of severity (van Os and Reininghaus 2016), in which the connectivity of various symptoms increases as socio-environmental load increases, thus leading to more symptoms and increased illness severity (Guloksuz et al. 2015). This network model of psychopathology will be described in greater detail later in this chapter. To date, no study examined such a transdiagnostic network model of psychopathology for the outcome self-directed violence. Therefore, prospective studies cutting across the boundaries of distinct, categorical, classification systems such as the Diagnostic and Statistical Manual of Mental Disorders (American Psychiatric Association 2013) are needed to gain further understanding of the interplay between psychotic experiences and non-psychotic psychopathology on the pathway to selfdirected violence.

Second, the meta-analysis presented in chapter 2 showed an association between psychotic experiences and non-suicidal self-injurious behaviour. This pooled odds ratio was based on one longitudinal study (Martin et al. 2015) and two cross-sectional studies (Nishida et al. 2010; Nishida et al. 2014). The longitudinal study showed that psychotic experiences were only associated with non-suicidal self-injurious behaviour if the participant reported also psychological distress, while psychotic experiences in absence of psychological distress were not associated with non-suicidal self-injurious behaviour (Martin et al. 2015). This finding supports the hypothesis that the association between 
psychotic experiences and self-directed violence is non-specific and reflects the increased risk of non-suicidal behaviour in individuals with non-psychotic psychopathology. However, the association between psychotic experiences and non-suicidal selfinjurious behaviour could not be examined in the datasets used in chapter 3 , because there was no data included about non-suicidal self-injurious behaviour. Therefore, more longitudinal research is required to examine the association between psychotic experiences and non-suicidal self-injurious behaviour while adjusting for dimensional nonpsychotic psychopathology.

Third, although the results presented in chapter 3 have shown that psychotic experiences are associated with suicide attempts in individuals with a non-psychotic mental disorder, even after adjustment for non-psychotic psychopathology, the role of psychotic experiences in the ideation-to-action framework of suicidality (May and Klonsky 2016) remains unclear. According to the ideation-to-action framework, the development of suicidal ideation and the progression of suicidal ideation to suicidal behaviour are distinct phenomena with different risk factors and underlying mechanisms (Klonsky et al. 2016). Although many studies examined risk factors for suicidal ideation and suicidal behaviour, few examined the differences between those with suicidal ideation and those who attempt suicide (Burke and Alloy 2016). Therefore, risk factors for suicide attempts in individuals with suicidal ideation remain largely unknown (May and Klonsky 2016). Since the results in chapter 3 showed that in individuals with mental disorders, psychotic experiences are a risk factor for suicide attempts, psychotic experiences are a candidate predictor in the ideation-to-action framework of suicidality. However, in the present analyses, this research question was not examined.

In conclusion, chapters 2 and 3 of this thesis showed evidence for a longitudinal association between psychotic experiences and self-directed violence. However, psychotic experiences were only associated with self-directed violence if they co-occurred with non-psychotic psychopathology, and the exact mechanisms through which psychotic experiences and non-psychotic symptoms interact in this model remain unknown.

\section{Psychotic experiences and interpersonal violence perpetration}

After examining the association between psychotic experiences and self-directed violence, chapter 4 examined the association between psychotic experiences and interpersonal violence perpetration. For many years, researchers examined the link between various mental disorders and violence perpetration, showing that various mental disorders (Fazel et al. 2010; Dowden and Brown 2002; Yu et al. 2012; Fazel et al. 2015) including psychotic disorder (Douglas et al. 2009; Fazel et al. 2009; Large and Nielssen 2011) are associated with an increased risk of violence perpetration. In contrast to the numerous studies about psychotic disorder and interpersonal violence perpetration, studies on the association between psychotic experiences and interpersonal violence 
perpetration are scarce. Available studies in the general population showed a crosssectional association between psychotic experiences on the one hand, and interpersonal violence (Kinoshita et al. 2011; Mojtabai 2006; Swanson et al. 1996; Nederlof et al. 2012; Coid et al. 2016) and arrest on the other (Mojtabai 2006). However, since causality cannot be inferred from cross-sectional studies, a longitudinal study on these associations was conducted. More specifically, using a prospective, general population sample (de Graaf et al. 2010), associations between baseline psychotic experiences and incident physical violence, psychological violence and arrest were examined (Honings et al. 2016c).

Results showed that hallucinations and delusions were differentially associated with the various outcomes. Physical violence perpetration was predicted by presence of hallucinations, but not by delusions. This finding is inconsistent with the 'threat/controloverride' hypothesis of violence in psychosis, which explains physical violence perpetration as a response to the threatening content of certain delusions. These delusions cause a person to believe that people are out to harm him or that outside forces are controlling their minds (Link et al. 1998; Rueve and Welton 2008; Swanson et al. 1996). The present study did not find an association between delusions and physical violence perpetration. However, statistical power was insufficient to test associations between specific psychotic experiences, such as the threat/control-override delusions, and physical violence perpetration. Previous literature reported contrasting results. A metaanalysis of seven cross-sectional population surveys found an association between paranoid ideation and violence perpetration, but no association between hallucinations and violence (Coid et al. 2016). Two other studies found that both hallucinations and delusions were associated with violence perpetration (Kinoshita et al. 2011; Mojtabai 2006), while another study found that only hallucinations were associated (Nederlof et al. 2012). Thus, although chapter 4 showed evidence for a link between psychotic experiences and violence perpetration, longitudinal research is scarce, and more research is needed in order to test longitudinal associations between specific psychotic experiences and violence perpetration.

In addition to the association with physical violence perpetration, results presented in chapter 4 showed that psychotic experiences were also associated with psychological victimisation in the unadjusted analyses. However, none of the associations remained statistically significant after adjustment for demographics, dimensional psychopathology and contextual confounders such as childhood trauma and negative life events. Thus, the association between psychotic experiences and psychological violence perpetration is non-specific and is confounded by non-psychotic psychopathology. No previous study examined the link between psychotic experiences and psychological violence perpetration. Therefore, results of the present analyses cannot be compared to previous research.

Finally, the association between psychotic experiences and incident arrest was examined as well in chapter 4 . Results showed that delusions were associated with an 
increased risk of arrest, while hallucinations were not. Only one previous study examined the association between psychotic experiences and arrest, showing that psychotic experiences were associated with arrest for both aggravated assault and other illegal behaviours (Mojtabai 2006). However, psychotic experiences were not specified into delusions and hallucinations in this study, thus impeding comparison of results.

In conclusion, results of the study presented in chapter 4 show that individuals with psychotic experiences are at increased risk of violence perpetration and arrest. However, the underlying mechanisms are unknown. Moreover, it remains unclear why hallucinations and delusions are differentially associated with the various outcomes of this study. It is possible that individuals with psychotic experiences, for example paranoid delusions, are more likely to interpret social interactions negatively. Since stressreactivity is increased in individuals with psychotic experiences (Myin-Germeys et al. 2001), this negative interpretation of social interaction could lead to increased levels of stress and violence perpetration. In addition, it is possible that the association between psychotic experiences and violence perpetration is confounded. A previous study reported that comorbid psychopathology, and in particular antisocial personality disorder and substance abuse, were key to understanding the association between psychotic experiences and violence in the general population (Coid et al. 2016). Similarly, the association between schizophrenia and violence perpetration was strongly attenuated after adjustment for substance abuse (Fazel et al. 2009). In the present analyses, hallucinations and delusions remained associated with physical violence and arrest, respectively, even after adjustment for various confounders, including non-psychotic psychopathology, substance use and antisocial personality traits. However, in agreement with previous research, adjustment for confounders decreased effect sizes. Although the present study provides the first evidence for a longitudinal association between psychotic experiences and violence perpetration, more longitudinal research is required in order to confirm the results. Future research should examine the association between specific psychotic experiences and violence perpetration, since statistical power was insufficient for such analyses in the present study. Moreover, the present analyses should be repeated in other longitudinal, general population datasets to examine whether the finding that hallucinations and delusions are differentially associated with physical violence perpetration, psychological violence perpetration and arrest, can be replicated. Finally, the role of non-psychotic psychopathology in the association between psychotic experiences and violence perpetration needs further clarification.

\section{Psychotic experiences and violence victimisation}

In chapter 5, the associations between psychotic experiences and victimisation across the life course were examined. Although chapter 4 focussed on psychotic experiences and interpersonal violence perpetration, the link between psychotic experiences and 
interpersonal violence victimisation warrants a separate analysis, since violence victimisation is highly prevalent among individuals with psychotic disorders (Bebbington et al. 2004), both during childhood (Kelleher et al. 2013b; Morgan and Gayer-Anderson 2016; Read et al. 2005) and during adulthood (de Mooij et al. 2015; Hart et al. 2012; Kamperman et al. 2014). Moreover, there is evidence that individuals with schizophrenia are approximately fourteen times more likely to be victims of violence than perpetrators (Brekke et al. 2001).

The link between childhood victimisation and psychosis has been well-established in the literature: childhood victimisation increases the risk of both full-blown psychosis (Read et al. 2005; Morgan and Gayer-Anderson 2016) and psychotic experiences (Kelleher et al. 2013b). The association between psychosis and adult victimisation is less clear, although available evidence shows that the prevalence of violence victimisation is high among individuals with psychotic disorder and other severe mental illnesses (de Mooij et al. 2015; Hart et al. 2012; Kamperman et al. 2014). However, the temporal sequence of the association between psychosis and adult victimisation remains unknown, since longitudinal evidence was scarce. Therefore, a longitudinal study on the bidirectional association between psychotic experiences and adult victimisation was conducted, while simultaneously assessing the moderating effect of childhood victimisation.

Results of the study presented in chapter 5 showed that both isolated childhood victimisation and isolated psychotic experiences were associated with an increased risk of adult victimisation (Honings et al. 2017). However, contrary to the hypothesis that childhood victimisation and psychotic experiences would act synergistically to increase the risk of adult victimisation, results showed that the co-occurrence of psychotic experiences with childhood victimisation was associated with a lower risk of adult victimisation than isolated psychotic experiences or childhood victimisation. Thus, there was evidence of a negative interaction effect pointing towards parallelism (Darroch 1997; Murray et al. 2002). This means that childhood victimisation and psychotic experiences are competing risk factors for adult victimisation. In other words, psychotic experiences are no longer a risk factor for adult victimisation if childhood victimisation has caused adult victimisation, and vice versa. No previous, longitudinal study examined the association between psychotic experiences and incident adult victimisation. Therefore, the finding that childhood victimisation and psychotic experiences are competing risk factors for adult victimisation should be replicated in future research.

Furthermore, the results of the study presented in chapter 5 showed that childhood victimisation and adult victimisation were both associated with incident psychotic experiences (Honings et al. 2017). This finding is consistent with previous research (Bebbington et al. 2011; Lataster et al. 2012; Morgan et al. 2014). The results of the present study showed no positive interaction between childhood victimisation and adult victimisation when studying the outcome incident psychotic experiences. This suggests that both forms of victimisation are independent, cumulative risk factors. Furthermore, it suggests that there are two pathways from childhood victimisation to adult psychotic 
experiences: one direct, and one indirect through adult victimisation. Previous studies reported positive interactions between childhood victimisation and adult victimisation for the outcome psychotic experiences (Bebbington et al. 2011; Lataster et al. 2012; Morgan et al. 2014). However, a wide variety of definitions of childhood victimisation and adult victimisation were used in these studies, thus impeding direct comparison.

In conclusion, the study presented in chapter 5 provided evidence that psychotic experiences and victimisation are interconnected throughout the life course, resulting in a complex interplay in which childhood victimisation and adult victimisation lead to both psychotic experiences and re-victimisation. Although chapter 5 presents the first evidence for a bidirectional association between psychotic experiences and adult victimisation in individuals without childhood victimisation, and for two pathways from childhood victimisation to adult psychotic experiences, many questions remain unanswered.

The study presented in chapter 5 examined violence victimisation by an intimate partner. Although violence victimisation often occurs at home (i.e. domestic violence victimisation) (Oram et al. 2013), longitudinal evidence on the association between psychotic experiences and violence victimisation by other persons is scarce. Future research should examine associations between psychotic experiences and violence victimisation by any person in general.

It is unclear why individuals with psychotic experiences are at increased risk of adult victimisation. It could be hypothesized that the increased risk of adult victimisation in individuals with psychotic experiences results from disordered behaviour, for example arising from paranoid delusions, leading to social conflict and victimisation. Another possible explanation is that individuals with psychotic experiences are more likely to report adult victimisation, although evidence shows that individuals with psychosis provide reliable reports of victimisation (Goodman et al. 1999; Fisher et al. 2011). Since non-psychotic mental disorders are prevalent among individuals with psychotic experiences (Kelleher et al. 2012), and adult victimisation is prevalent among several mental disorders (Latalova et al. 2014), future research should also attempt to determine the specificity of the association between psychotic experiences and adult victimisation and unravel the interaction between psychotic and non-psychotic symptomatology on the pathway to adult victimisation. Various explanations have been suggested in the literature why individuals with both psychotic and non-psychotic mental disorders are at increased risk of victimisation. First, it has been suggested that individuals with mental disorders have an impaired ability to manage conflict situations due to their illness, thus resulting in a higher risk of victimisation (Bellack et al. 1992). The impaired ability to manage conflict situations in individuals with mental disorders could also result in an increased risk of violence perpetration; violence victimisation could then occur in reaction to the violence perpetration. Evidence supports this hypothesis by showing a relationship between violence victimisation and violence perpetration in individuals with mental disorders (Latalova et al. 2014). Second, it is possible that individuals with mental disorders experience more violence victimisation, because they more often live in 
socially disadvantaged neighbourhoods characterized by high levels of violence and crime (Logdberg et al. 2004).

The pathway from childhood victimisation to psychotic experiences, either direct or indirect through adult victimisation, needs further examination as well, since previous studies were inconclusive. There is evidence that research using the experience sampling method (Delespaul 1995), which examines moment-to-moment changes in mental states several times a day, offers new perspectives on the interplay between environmental factors and psychosis (Glaser et al. 2006). Therefore, the experience sampling method could be used to further elucidate the mechanisms underlying the association between psychosis and victimisation. This suggestion will be discussed later in this chapter.

\section{An integrated perspective on psychosis and multidirectional violence}

The present thesis demonstrated that individuals with psychotic experiences are at increased risk of self-directed violence, interpersonal violence perpetration and interpersonal violence victimisation. Although these different forms of violence were analysed in the present thesis as distinctive phenomena, in reality they are intricately interconnected. Studies show that individuals who experience victimisation during childhood are at increased risk of adult violence re-victimisation (Desai et al. 2002), violence perpetration (Maas et al. 2008) and suicide attempts (Dube et al. 2001). Similarly, adult victimisation is associated with suicidal behaviour (Palm et al. 2016) and interpersonal violence perpetration (Desmarais et al. 2014). Moreover, individuals who perpetrate interpersonal violence are more likely to display suicidal behaviour (Evans et al. 2001). Since psychotic experiences are associated with all these outcomes, a shared, underlying mechanism could be hypothesized.

A common, traditional conceptualization of the aetiology of various mental disorders including schizophrenia, is the diathesis-stress model (Monroe and Simons 1991). The diathesis-stress model in psychiatry originates from early schizophrenia research (Meehl 1962). According to this theory, all individuals have a certain diathesis or predisposition for psychopathology, both biologically and psychologically. However, psychopathology only becomes manifest if a combination of stressors exceeds a threshold determined by the individual's vulnerability. Thus, the diathesis-stress model is a biopsychosocial model, integrating gene-environment interactions (Ingram and Luxton 2005). There is now accumulating evidence that psychosocial stress is essential to the development of psychotic symptoms (van Winkel et al. 2008). Consistent with the results presented in chapter 5 of this thesis, it has been shown that cumulative exposure to traumatic life events increases the risk of psychosis (Shevlin et al. 2008). Other environmental, psychosocial stressors associated with an increased risk of psychosis include urbanicity (van Os et al. 2004) and 'social defeat' (i.e. outsiders status) (Selten and Cantor-Graae 2007; Selten et al. 2013). However, the mechanism linking stress to psy- 
chosis is not completely clear. Evidence has shown that individuals with psychosis have an increased stress-reactivity, i.e. they report stronger increased emotional reactivity to daily life stress (Myin-Germeys et al. 2001), thus suggesting that the association between psychosis and stress is the consequence of an underlying vulnerability or diathesis (Myin-Germeys et al. 2001). This increase in emotional reactivity in individuals with psychosis can possibly be explained by a process called sensitization (Collip et al. 2008), defined by van Winkel et al. (2008) as "a process in which repeated exposure to a certain event increases the behavioural and biological response to later exposure to a similar event, even if the later exposure is not as severe".

Several biological mechanisms underlying this mechanism of stress-reactivity in psychosis have been hypothesized. An important component in this mechanism is the hypothalamic-pituitary-adrenal (HPA) axis, which is responsible for the biological stress response through the secretion of cortisol. There is evidence of HPA-axis dysfunction in individuals with psychosis, showing that individuals with psychosis have increased baseline levels of cortisol (Bradley and Dinan 2010; Pruessner et al. 2017), attenuated cortisol awakening responses (Berger et al. 2016) and blunted cortisol responses in reaction to stress (Ciufolini et al. 2014). Several environmental risk factors for psychosis, such as childhood victimisation, have been associated with increased HPA-axis activity (Heim et al. 2008). Moreover, multiple genes impacting on the HPA-axis have been identified as moderators for the risk of psychosis (van Winkel et al. 2008). Heightened HPA-axis activity results in increased activity of dopaminergic pathways, which has been associated with the pathophysiology of psychosis (Walker et al. 2008). However, evidence also shows that HPA-axis dysregulation is not only associated with psychosis, but also with various other mental disorders and mental health outcomes, including depression (Lopez-Duran et al. 2009), bipolar disorder (Belvederi Murri et al. 2016), anxiety disorders (Faravelli et al. 2012), suicidal behaviour (Jokinen and Nordstrom 2009) and violence perpetration (Gowin et al. 2013). Thus, HPA-axis dysregulation, a core biological mechanism assumed to mediate the diathesis-stress model of psychosis, is not specific for psychosis. Instead, HPA-axis dysregulation, and therefore impaired stress regulation, is associated with all psychopathology.

In the DSM, mental disorders are depicted as discrete, independent disorders. Although this approach was necessary in order to develop a consistent language and standard criteria for mental disorders, this conceptualization of mental disorders is not in accordance with recent evidence. Research has shown that many mental disorders are longitudinally and phenomenologically continuous. For example, psychotic disorders (Linscott and van Os 2013), depression (Judd et al. 2002), bipolar disorder (Tijssen et al. 2010) and autism (Constantino and Todd 2003) can be traced back to subthreshold experiences in the general population, while sharing genetic and non-genetic causes with the respective full-blown disorder (Lundstrom et al. 2012; Binbay et al. 2012; Kendler and Gardner 1998). Moreover, evidence shows that the core symptoms of various mental disorders, for example psychotic symptoms in individuals with schizo- 
phrenia, are not disorder-specific, but are prevalent in many other mental disorders (Kelleher and DeVylder 2017). Similarly, depression symptoms are common in schizophrenia and other mental disorders (Gozdzik-Zelazny et al. 2011). Thus, it has been suggested that mental disorders do not represent discrete disease entities. Instead, psychopathology occurs transdiagnostically (Nolen-Hoeksema and Watkins 2011), thereby offering an explanation for the extremely high comorbidity of mental disorders (Maj 2005). There is evidence that the earliest expressions of psychopathology are nonspecific, but consist of a mixture of anxiety, depression, psychosis and other symptoms that dynamically affect each other over time, forming a causal network (van Os 2013). Environmental factors, such as childhood victimisation, have also been associated with an admixture of psychosis, anxiety and depression symptoms (van Nierop et al. 2015). Recently, network models have been developed in order to study the way symptoms dynamically affect each other (Borsboom and Cramer 2013). In this conceptualization of mental disorders, psychopathology arises from the interaction between symptoms (Fig. 1) (Isvoranu et al. 2016). Vulnerability is represented in the network as differences in connectivity. Thus, if symptom dimensions are stronger interconnected, symptoms can activate other symptoms more easily, thereby rendering the system less resilient (Isvoranu et al. 2016). Network models have been examined for various mental health outcomes described in this thesis, including psychosis (Isvoranu et al. 2016), trauma (Isvoranu et al. 2017) and suicidal behaviour (de Beurs 2017).

An integrated perspective on psychosis and multidirectional violence can be achieved by combining the traditional diathesis-stress model of psychopathology with the new network approach of psychopathology. Figure 2 shows a visualization of such an integrated perspective. As described earlier, the diathesis-stress model is based on the assumption that every individual has an individual vulnerability, resulting from multiple factors including genetic predisposition, psychological factors and sociodemographic factors. This vulnerability or diathesis is continuous, as shown by the continuous distribution of various symptom dimensions in the general population (Linscott and van Os 2013; Judd et al. 2002; Tijssen et al. 2010) and changes dynamically over time. In this thesis, adult victimisation and child victimisation were identified as cumulative risk factors for psychosis. Thus, victimisation operates as a stressor in the diathesisstress model, leading to general psychopathology symptoms (Isvoranu et al. 2017). However, victimisation also increases the vulnerability for psychopathology by increasing the responsiveness of the HPA-axis through sensitization (Collip et al. 2008; van Winkel et al. 2008). Thus, in individuals with a certain diathesis, victimisation or other stressors may lead to a 'general distress syndrome' (McGorry and van Os 2013; van Os 2013), in which various symptom dimensions affect each other dynamically, resulting in a complex network (Fig. 1). Recent studies on trauma and psychosis using network models showed that childhood trauma was not directly associated with psychotic symptoms, but only via non-psychotic symptoms (Isvoranu et al. 2017), thus supporting the construct of an affective pathway to psychosis (Myin-Germeys and van Os 2007). More- 
over, exposure to environmental factors such as victimisation was associated with a more strong and subsequently more vulnerable psychopathology network (Isvoranu et al. 2016), thereby showing that victimisation operates in the diathesis-stress model as both stressor and modifier of the diathesis. Psychotic experiences have been identified as an indicator of severity in non-psychotic psychopathology (Armando et al. 2013; Guloksuz et al. 2015). Thus, psychotic experiences occur through a complex interaction with non-psychotic psychopathology (van Rossum et al. 2011), caused by environmental stressors such as victimisation.

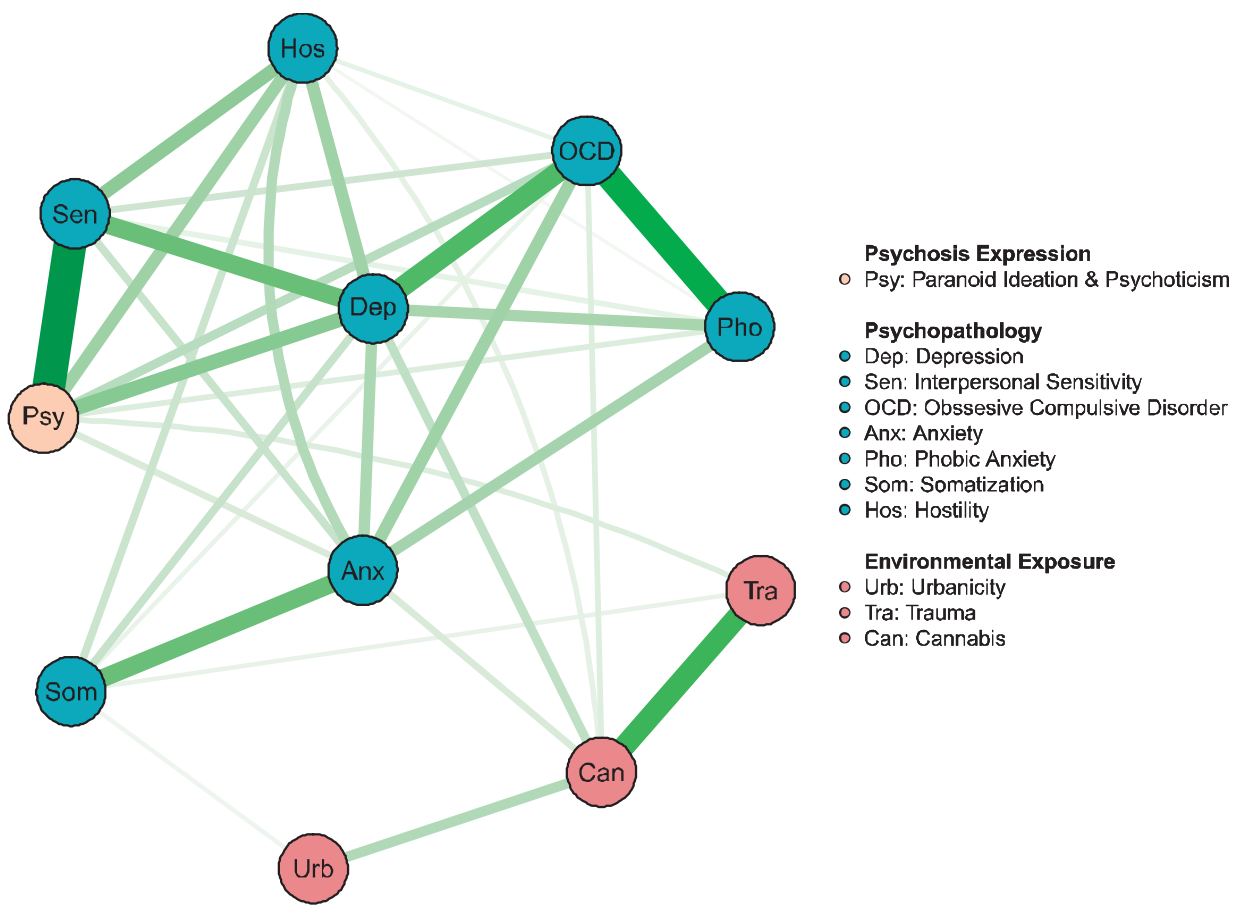

Figure 1: Example of a network visualization of environmental factors and both psychotic and non-psychotic symptoms. Adapted from "A Network Approach to Environmental Impact in Psychotic Disorder: Brief Theoretical Framework", by A.M. Isvoranu, D. Borsboom, J. van Os and S. Guloksuz, 2016, Schizophrenia Bulletin, 42, p. 871. Copyright by the authors. Adapted with permission.

The results of this thesis support this model based on both the diathesis-stress model and the network approach of psychopathology. Results presented in chapters 2 and 3 showed that the link between psychotic experiences and self-directed violence was confounded by non-psychotic psychopathology: after adjustment for mania, depression and anxiety symptoms, there was no longer an association between psychotic experiences and self-directed violence. Thus, psychotic experiences increased the risk of selfdirected violence through interaction with non-psychotic psychopathology. Similarly, the association between psychotic experiences and violence perpetration was also 
confounded by non-psychotic psychopathology (chapter 4). A previous network study found that poor impulse control was associated with psychosis (Isvoranu et al. 2017). Thus, impulsivity could be an important factor in the associations between psychotic experiences and self-directed violence and violence perpetration (Chapman et al. 1984). Results of chapter 5 showed that psychotic experiences and child victimisation were competing risk factors for adult victimisation. It is possible that psychotic experiences and child victimisation both increase the risk of adult victimisation through increasing general non-psychotic psychopathology, thus making them competing risk factors. However, this hypothesis has not been investigated yet. Literature shows that suicidal behaviour, violence perpetration and violence victimisation are interrelated (Evans et al. 2001). This finding is likely the result from the hypothesized, underlying network of both psychotic and non-psychotic psychopathology.

In conclusion, the results presented in this thesis can be integrated by combining the traditional diathesis-stress model with the newer network approach of psychopathology into one model. Although this thesis focussed on the associations between psychosis and multidirectional violence, it is hypothesized that psychosis is connected to multidirectional violence through a complex interplay with non-psychotic psychopathology. However, more research is needed to investigate this hypothesis.

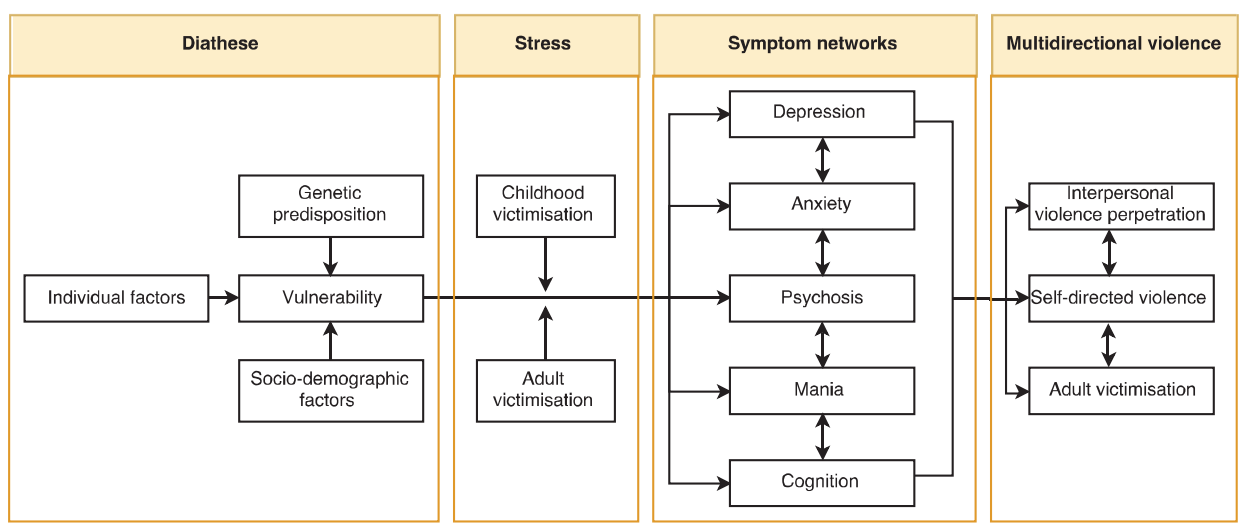

Figure 2: An integrated perspective on psychosis and multidirectional violence combining the diathesis-stress model of psychopathology with the network approach of psychopathology. 


\section{Limitations of the present study}

The studies presented in this thesis had some methodological limitations, which were described in detail in the previous chapters. Two important limitations were present in all studies of this thesis.

First, associations between psychosis and multidirectional violence were only examined for positive symptoms (i.e. delusions and hallucinations). Previous studies showed that negative symptoms are also present at the subthreshold level in the general population, and have been associated with poor mental health outcomes (Dominguez et al. 2010; Werbeloff et al. 2015). Therefore, an association between negative symptoms and multidirectional violence could be hypothesized. Unfortunately, these associations could not be examined in this thesis since the used datasets did not contain information about negative symptoms. Future studies should examine associations between negative psychotic symptoms and multidirectional violence.

Second, the incidence of the outcomes studied in this thesis was low. Suicide attempts, violence perpetration and violence victimisation are, fortunately, rare in general population samples. For the outcome suicide attempts this limitation could be overcome by combining several general population samples into one large dataset. However, for the outcomes violence perpetration and violence victimisation this was not possible. The dataset used for the analyses on violence perpetration and victimisation (i.e. NEMESIS-2), included 6646 individuals at baseline (de Graaf et al. 2010). Nevertheless, statistical power was low in some of the analyses with violence perpetration and victimisation as outcome. Therefore, epidemiological studies in the general population on these outcomes are naturally limited by the low incidence of violence perpetration and victimisation. In order to investigate the associations identified in this thesis further, a different methodology could be applied in future studies.

\section{Directions for future research}

In this chapter, the network approach was introduced as a method to study psychosis in relation to non-psychotic symptom dimensions. Although network models of psychosis have been studied, network models of multidirectional violence have not been examined. Recently, the network approached has been identified as a useful approach to study suicidal behaviour, and researchers are currently re-analysing existing data using network analysis (de Beurs 2017). Thus, network models of suicidal behaviour should emerge soon, hopefully offering new insights into the mechanisms of suicidal behaviour. The role of psychotic experiences could be examined in these models as well.

Although this thesis identified associations between psychotic experiences and multidirectional violence, predicting violence remains difficult. In forensic psychiatry, various risk assessment instruments have been developed in order to identify individuals at 
high risk for violence. However, the predictive accuracy of these instruments is low (Fazel et al. 2012). A recent report on transdiagnostic factors in forensic psychiatry concluded that the dynamic systems theory offers new starting points for research on the prediction of violence (van Os et al. 2015). Models based on the dynamic systems theory are similar to the previously mentioned network models. In these models, critical transitions, for example the occurrence of a depressive episode, were predicted by a critical slowing down of the system (van de Leemput et al. 2014; Wichers et al. 2016). It was hypothesized that dynamic system models could be used to predict violence by observing a critical slowing down (van Os et al. 2015). To date, only one study examined a dynamic system model to predict interpersonal violence perpetration (Odgers et al. 2009). In this study, patients at risk of violence were followed during twenty-six weeks, administering the Brief Symptom Inventory weekly. Next, the symptom scores were used to generate graphs depicting the temporal fluctuations of symptoms. Results of this study showed that patterns of rapid symptom fluctuation and symptom amplification were associated with the occurrence of violence (Odgers et al. 2009). Thus, this study presents evidence that dynamic system models can potentially be used to predict interpersonal violence perpetration. However, no previous study used dynamic system models to predict self-directed violence. Moreover, the accuracy of such models could be improved by increasing the assessment frequency, for example by using momentary assessment techniques such as the experience sampling method (Delespaul 1995).

The experience sampling method has been widely used to study psychosis (MyinGermeys et al. 2003). In addition, experience sampling has been used to study suicidal behaviour as well (Palmier-Claus et al. 2012; Nock et al. 2009; Thompson et al. 2014). One previous study applying the experience sampling method to study intimate partner violence victimisation showed that the experience sampling method is a promising approach (Sullivan et al. 2011). However, to date, no experience sampling studies examining psychotic experiences and multidirectional violence have been published. Although the experience sampling method could offer interesting insights in the interplay of psychotic experiences with other non-psychotic symptom dimensions, studying the association between psychotic experiences and violence using the experience sampling method is difficult, since the outcomes, such as suicidal behaviour and interpersonal violence, are rare. Therefore, case-control study designs could be used to explore differences in network models between individuals with and without suicidal ideation or behaviour. Similarly, studies could be designed to examine differences in network models in individuals exposed and unexposed to childhood or adult victimisation. The role of psychotic experiences in predicting violence could be prospectively examined using the experience sampling method in individuals in forensic psychiatric clinics. Another option to overcome the problem that violence outcomes are rare would be to use proxies for suicidal ideation or violence in experience sampling studies. For example, hopelessness can be measured in experience sampling studies and is strongly associated with suicidal behaviour (Beck et al. 1985) and violence perpetration (Bolland et al. 2001). 
In conclusion, more longitudinal research is required in order to understand the mechanisms underlying the associations between psychotic experiences and multidirectional violence. Network approaches, dynamic system models and experience sampling offer possibilities to gain new insights into these associations.

\section{Conclusions and implications}

The aim of this thesis was to explore the associations between psychosis and multidirectional violence in the general population and to assess whether associations between psychotic experiences and self-directed violence, interpersonal violence perpetration and interpersonal violence victimisation were analogous to the associations between full-blown psychosis and these outcomes. Results showed that individuals were at increased risk of self-directed violence (chapter 2 and 3) and interpersonal violence perpetration (chapter 4). Moreover, there was a bidirectional association between psychotic experiences and adult victimisation (chapter 5). Previous studies reported that individuals with full-blown psychotic disorder were at increased risk of self-directed violence (Hor and Taylor 2010), interpersonal violence perpetration (Fazel et al. 2009) and interpersonal violence victimisation (de Mooij et al. 2015; Hart et al. 2012; Kamperman et al. 2014). Thus, associations between psychotic experiences and multidirectional violence were analogous to the associations between full-blown psychotic disorder and multidirectional violence and, thereby, the results of this thesis support the hypothesis of an extended psychosis phenotype in the general population (van Os and Linscott 2012).

Results of this thesis are in agreement with previous studies that identified psychotic experiences as an indicator of severity in non-psychotic psychopathology (Guloksuz et al. 2015). This finding has several clinical implications. Most importantly, this finding highlights the importance of a careful assessment of psychotic symptoms in all individuals presenting to mental health care, since psychotic experiences are associated with both self-directed and interpersonal violence. Especially in individuals with nonpsychotic psychopathology, thorough assessment of psychotic experiences is important, since psychotic experiences are strongly associated with suicide attempts in these individuals. In addition, the risk of interpersonal violence perpetration should be considered in all individuals presenting themselves with psychotic symptoms in absence of a psychotic disorder, since psychotic experiences were identified as a risk factor for violence perpetration and arrest. Finally, clinicians should be aware that both childhood victimisation and adult victimisation are prevalent in individuals with psychotic experiences and that these factors are associated with poor mental health outcomes. Therefore, screening for victimisation in adults with psychotic experiences is important in order to identify victims of violence and offer adequate treatment. 
The thesis started with an introduction in which a historical overview of psychosis was given. In this overview it was shown that the concept of psychosis has developed throughout the centuries. Whereas psychosis was used as a synonym for insanity in the $19^{\text {th }}$ century, recent evidence has shown that psychosis exists as a prevalent extended phenotype in the general population. However, stigmatisation is still highly prevalent among individuals with mental disorders (van Zelst 2009) and individuals with psychotic disorders are still regularly regarded as dangerous and violent (Owen 2012; Crisp et al. 2000). Studies have shown that individuals with psychotic disorder indeed are at increased risk of self-directed and interpersonal violence perpetration. However, studies have also shown that individuals with schizophrenia are more likely to be victims of violence than perpetrators (Brekke et al. 2001). The present thesis showed that the risk of multidirectional violence in individuals with psychosis can be traced back to the general population. Thus, the common stereotype that individuals with mental illness are dangerous, should be dismissed, since the increased risk of violence is present in a substantial group of general population individuals as well, sharing a common, developmental phenotype characterized by psychotic experiences. 


\section{References}

American Psychiatric Association 2013. Diagnostic and statistical manual of mental disorders, Washington, DC, American Psychiatric Association.

Armando, M., Lin, A., Girardi, P., Righetti, V., Dario, C., Saba, R., Decrescenzo, F., Mazzone, L., Vicari, S., Birchwood, M. \& Fiori Nastro, P. 2013. Prevalence of psychotic-like experiences in young adults with social anxiety disorder and correlation with affective dysregulation. J Nerv Ment Dis, 201, 1053-9.

Bebbington, P., Jonas, S., Kuipers, E., King, M., Cooper, C., Brugha, T., Meltzer, H., Mcmanus, S. \& Jenkins, R. 2011. Childhood sexual abuse and psychosis: data from a cross-sectional national psychiatric survey in England. BrJ Psychiatry, 199, 29-37.

Bebbington, P. E., Bhugra, D., Brugha, T., Singleton, N., Farrell, M., Jenkins, R., Lewis, G. \& Meltzer, H. 2004. Psychosis, victimisation and childhood disadvantage: evidence from the second British National Survey of Psychiatric Morbidity. Br J Psychiatry, 185, 220-6.

Beck, A. T., Steer, R. A., Kovacs, M. \& Garrison, B. 1985. Hopelessness and eventual suicide: a 10-year prospective study of patients hospitalized with suicidal ideation. Am J Psychiatry, 142, 559-63.

Bellack, A. S., Mueser, K. T., Wade, J., Sayers, S. \& Morrison, R. L. 1992. The ability of schizophrenics to perceive and cope with negative affect. Br J Psychiatry, 160, 473-80.

Belvederi Murri, M., Prestia, D., Mondelli, V., Pariante, C., Patti, S., Olivieri, B., Arzani, C., Masotti, M., Respino, M., Antonioli, M., Vassallo, L., Serafini, G., Perna, G., Pompili, M. \& Amore, M. 2016. The HPA axis in bipolar disorder: Systematic review and meta-analysis. Psychoneuroendocrinology, 63, 327-42.

Berger, M., Kraeuter, A. K., Romanik, D., Malouf, P., Amminger, G. P. \& Sarnyai, Z. 2016. Cortisol awakening response in patients with psychosis: Systematic review and meta-analysis. Neurosci Biobehav Rev, 68, 157-166.

Bevan Jones, R., Thapar, A., Lewis, G. \& Zammit, S. 2012. The association between early autistic traits and psychotic experiences in adolescence. Schizophr Res, 135, 164-9.

Binbay, T., Drukker, M., Elbi, H., Tanik, F. A., Ozkinay, F., Onay, H., Zagli, N., Van Os, J. \& Alptekin, K. 2012. Testing the psychosis continuum: differential impact of genetic and nongenetic risk factors and comorbid psychopathology across the entire spectrum of psychosis. Schizophr Bull, 38, 992-1002.

Bolland, J. M., Mccallum, D. M., Lian, B., Bailey, C. J. \& Rowan, P. 2001. Hopelessness and violence among inner-city youths. Matern Child Health J, 5, 237-44.

Borsboom, D. \& Cramer, A. O. 2013. Network analysis: an integrative approach to the structure of psychopathology. Annu Rev Clin Psychol, 9, 91-121.

Bradley, A. J. \& Dinan, T. G. 2010. A systematic review of hypothalamic-pituitary-adrenal axis function in schizophrenia: implications for mortality. J Psychopharmacol, 24, 91-118.

Brekke, J. S., Prindle, C., Bae, S. W. \& Long, J. D. 2001. Risks for individuals with schizophrenia who are living in the community. Psychiatr Serv, 52, 1358-66.

Burke, T. A. \& Alloy, L. B. 2016. Moving Toward an Ideation-to-Action Framework in Suicide Research: A Commentary on. Clin Psychol (New York), 23, 26-30.

Calkins, M. E., Moore, T. M., Merikangas, K. R., Burstein, M., Satterthwaite, T. D., Bilker, W. B., Ruparel, K., Chiavacci, R., Wolf, D. H., Mentch, F., Qiu, H., Connolly, J. J., Sleiman, P. A., Hakonarson, H., Gur, R. C. \& Gur, R. E. 2014. The psychosis spectrum in a young U.S. community sample: findings from the Philadelphia Neurodevelopmental Cohort. World Psychiatry, 13, 296-305.

Chapman, L. J., Chapman, J. P., Numbers, J. S., Edell, W. S., Carpenter, B. N. \& Beckfield, D. 1984. Impulsive nonconformity as a trait contributing to the prediction of psychotic-like and schizotypal symptoms. J Nerv Ment Dis, 172, 681-91.

Ciufolini, S., Dazzan, P., Kempton, M. J., Pariante, C. \& Mondelli, V. 2014. HPA axis response to social stress is attenuated in schizophrenia but normal in depression: evidence from a meta-analysis of existing studies. Neurosci Biobehav Rev, 47, 359-68.

Coid, J. W., Ullrich, S., Bebbington, P., Fazel, S. \& Keers, R. 2016. Paranoid Ideation and Violence: Metaanalysis of Individual Subject Data of 7 Population Surveys. Schizophr Bull, 42, 907-15. 
Collip, D., Myin-Germeys, I. \& Van Os, J. 2008. Does the concept of "sensitization" provide a plausible mechanism for the putative link between the environment and schizophrenia? Schizophr Bull, 34, 220-5.

Constantino, J. N. \& Todd, R. D. 2003. Autistic traits in the general population: a twin study. Arch Gen Psychiatry, 60, 524-30.

Crisp, A. H., Gelder, M. G., Rix, S., Meltzer, H. I. \& Rowlands, O. J. 2000. Stigmatisation of people with mental illnesses. Br J Psychiatry, 177, 4-7.

Darroch, J. 1997. Biologic synergism and parallelism. Am J Epidemiol, 145, 661-8.

De Beurs, D. 2017. Network Analysis: A Novel Approach to Understand Suicidal Behaviour. International Journal of Environmental Research and Public Health, 14, 219.

De Graaf, R., Ten Have, M. \& Van Dorsselaer, S. 2010. The Netherlands Mental Health Survey and Incidence Study-2 (NEMESIS-2): design and methods. Int J Methods Psychiatr Res, 19, 125-41.

De Mooij, L. D., Kikkert, M., Lommerse, N. M., Peen, J., Meijwaard, S. C., Theunissen, J., Duurkoop, P. W., Goudriaan, A. E., Van, H. L., Beekman, A. T. \& Dekker, J. J. 2015. Victimisation in adults with severe mental illness: prevalence and risk factors. Br J Psychiatry, 207, 515-22.

Delespaul, P. a. E. G. 1995. Assessing Schizophrenia in Daily Life: The Experience Sampling Method, UPM.

Desai, S., Arias, I., Thompson, M. P. \& Basile, K. C. 2002. Childhood victimization and subsequent adult revictimization assessed in a nationally representative sample of women and men. Violence Vict, 17, 639-53.

Desmarais, S. L., Van Dorn, R. A., Johnson, K. L., Grimm, K. J., Douglas, K. S. \& Swartz, M. S. 2014. Community violence perpetration and victimization among adults with mental illnesses. Am J Public Health, 104, 2342-9.

Devylder, J. E., Burnette, D. \& Yang, L. H. 2014. Co-occurrence of psychotic experiences and common mental health conditions across four racially and ethnically diverse population samples. Psychol Med, 44, 350313.

Dominguez, M. D., Saka, M. C., Lieb, R., Wittchen, H. U. \& Van Os, J. 2010. Early expression of negative/disorganized symptoms predicting psychotic experiences and subsequent clinical psychosis: a 10year study. Am J Psychiatry, 167, 1075-82.

Douglas, K. S., Guy, L. S. \& Hart, S. D. 2009. Psychosis as a risk factor for violence to others: a meta-analysis. Psychol Bull, 135, 679-706.

Dowden, C. \& Brown, S. L. 2002. The role of substance abuse factors in predicting recidivism: A meta-analysis. Psychology Crime \& Law, 8, 243-264.

Dube, S. R., Anda, R. F., Felitti, V. J., Chapman, D. P., Williamson, D. F. \& Giles, W. H. 2001. Childhood abuse, household dysfunction, and the risk of attempted suicide throughout the life span: findings from the Adverse Childhood Experiences Study. JAMA, 286, 3089-96.

Evans, W. P., Marte, R. M., Betts, S. \& Silliman, B. 2001. Adolescent suicide risk and peer-related violent behaviors and victimization. Journal of Interpersonal Violence, 16, 1330-1348.

Faravelli, C., Lo Sauro, C., Lelli, L., Pietrini, F., Lazzeretti, L., Godini, L., Benni, L., Fioravanti, G., Talamba, G. A., Castellini, G. \& Ricca, V. 2012. The role of life events and HPA axis in anxiety disorders: a review. Curr Pharm Des, 18, 5663-74.

Fazel, S., Gulati, G., Linsell, L., Geddes, J. R. \& Grann, M. 2009. Schizophrenia and violence: systematic review and meta-analysis. PLoS Med, 6, e1000120.

Fazel, S., Lichtenstein, P., Grann, M., Goodwin, G. M. \& Langstrom, N. 2010. Bipolar disorder and violent crime: new evidence from population-based longitudinal studies and systematic review. Arch Gen Psychiatry, 67, 931-8.

Fazel, S., Singh, J. P., Doll, H. \& Grann, M. 2012. Use of risk assessment instruments to predict violence and antisocial behaviour in 73 samples involving 24827 people: systematic review and meta-analysis. BMJ, 345, e4692.

Fazel, S., Wolf, A., Chang, Z., Larsson, H., Goodwin, G. M. \& Lichtenstein, P. 2015. Depression and violence: a Swedish population study. Lancet Psychiatry, 2, 224-32.

Fisher, H. L., Craig, T. K., Fearon, P., Morgan, K., Dazzan, P., Lappin, J., Hutchinson, G., Doody, G. A., Jones, P. B., Mcguffin, P., Murray, R. M., Leff, J. \& Morgan, C. 2011. Reliability and comparability of psychosis patients' retrospective reports of childhood abuse. Schizophr Bull, 37, 546-53. 
Fisher, H. L., Caspi, A., Poulton, R., Meier, M. H., Houts, R., Harrington, H., Arseneault, L. \& Moffitt, T. E. 2013. Specificity of childhood psychotic symptoms for predicting schizophrenia by 38 years of age: a birth cohort study. Psychol Med, 43, 2077-86.

Glaser, J. P., Van Os, J., Portegijs, P. J. \& Myin-Germeys, I. 2006. Childhood trauma and emotional reactivity to daily life stress in adult frequent attenders of general practitioners. J Psychosom Res, 61, 229-36.

Goodman, L. A., Thompson, K. M., Weinfurt, K., Corl, S., Acker, P., Mueser, K. T. \& Rosenberg, S. D. 1999. Reliability of reports of violent victimization and posttraumatic stress disorder among men and women with serious mental illness. J Trauma Stress, 12, 587-99.

Gowin, J. L., Green, C. E., Alcorn, J. L., 3rd, Swann, A. C., Moeller, F. G. \& Lane, S. D. 2013. The role of cortisol and psychopathy in the cycle of violence. Psychopharmacology (Berl), 227, 661-72.

Gozdzik-Zelazny, A., Borecki, L. \& Pokorski, M. 2011. Depressive symptoms in schizophrenic patients. Eur J Med Res, 16, 549-52.

Guloksuz, S., Van Nierop, M., Lieb, R., Van Winkel, R., Wittchen, H. U. \& Van Os, J. 2015. Evidence that the presence of psychosis in non-psychotic disorder is environment-dependent and mediated by severity of non-psychotic psychopathology. Psychol Med, 45, 2389-401.

Hart, C., De Vet, R., Moran, P., Hatch, S. L. \& Dean, K. 2012. A UK population-based study of the relationship between mental disorder and victimisation. Soc Psychiatry Psychiatr Epidemiol, 47, 1581-90.

Heim, C., Newport, D. J., Mletzko, T., Miller, A. H. \& Nemeroff, C. B. 2008. The link between childhood trauma and depression: insights from HPA axis studies in humans. Psychoneuroendocrinology, 33, 693-710.

Honings, S., Drukker, M., Groen, R. \& Van Os, J. 2016a. Psychotic experiences and risk of self-injurious behaviour in the general population: a systematic review and meta-analysis. Psychol Med, 46, 237-51.

Honings, S., Drukker, M., Van Nierop, M., Van Winkel, R., Wittchen, H. U., Lieb, R., Ten Have, M., De Graaf, R., Van Dorsselaer, S. \& Van Os, J. 2016b. Psychotic experiences and incident suicidal ideation and behaviour: Disentangling the longitudinal associations from connected psychopathology. Psychiatry Res, 245, 267275.

Honings, S., Drukker, M., Ten Have, M., De Graaf, R., Van Dorsselaer, S. \& Van Os, J. 2016c. Psychotic Experiences and Risk of Violence Perpetration and Arrest in the General Population: A Prospective Study. PLoS One, 11, e0159023.

Honings, S., Drukker, M., Ten Have, M., De Graaf, R., Van Dorsselaer, S. \& Van Os, J. 2017. The interplay of psychosis and victimisation across the life course: a prospective study in the general population. Soc Psychiatry Psychiatr Epidemiol.

Hor, K. \& Taylor, M. 2010. Suicide and schizophrenia: a systematic review of rates and risk factors. J Psychopharmacol, 24, 81-90.

Ingram, R. E. \& Luxton, D. D. 2005. Vulnerability-stress models. Development of psychopathology: A vulnerability-stress perspective, 32-46.

Isvoranu, A. M., Borsboom, D., Van Os, J. \& Guloksuz, S. 2016. A Network Approach to Environmental Impact in Psychotic Disorder: Brief Theoretical Framework. Schizophr Bull, 42, 870-3.

Isvoranu, A. M., Van Borkulo, C. D., Boyette, L. L., Wigman, J. T., Vinkers, C. H., Borsboom, D. \& Group, I. 2017. A Network Approach to Psychosis: Pathways Between Childhood Trauma and Psychotic Symptoms. Schizophr Bull, 43, 187-196.

Jahn, D. R., Devylder, J. E. \& Hilimire, M. R. 2016. Explanatory risk factors in the relations between schizotypy and indicators of suicide risk. Psychiatry Res, 238, 68-73.

Jeppesen, P., Clemmensen, L., Munkholm, A., Rimvall, M. K., Rask, C. U., Jorgensen, T., Larsen, J. T., Petersen, L., Van Os, J. \& Skovgaard, A. M. 2015. Psychotic experiences co-occur with sleep problems, negative affect and mental disorders in preadolescence. J Child Psychol Psychiatry, 56, 558-65.

Johns, L. C., Cannon, M., Singleton, N., Murray, R. M., Farrell, M., Brugha, T., Bebbington, P., Jenkins, R. \& Meltzer, H. 2004. Prevalence and correlates of self-reported psychotic symptoms in the British population. Br J Psychiatry, 185, 298-305.

Jokinen, J. \& Nordstrom, P. 2009. HPA axis hyperactivity and attempted suicide in young adult mood disorder inpatients. J Affect Disord, 116, 117-20. 
Judd, L. L., Schettler, P. J. \& Akiskal, H. S. 2002. The prevalence, clinical relevance, and public health significance of subthreshold depressions. Psychiatr Clin North Am, 25, 685-98.

Kamperman, A. M., Henrichs, J., Bogaerts, S., Lesaffre, E. M., Wierdsma, A. I., Ghauharali, R. R., Swildens, W., Nijssen, Y., Van Der Gaag, M., Theunissen, J. R., Delespaul, P. A., Van Weeghel, J., Van Busschbach, J. T., Kroon, H., Teplin, L. A., Van De Mheen, D. \& Mulder, C. L. 2014. Criminal victimisation in people with severe mental illness: a multi-site prevalence and incidence survey in the Netherlands. PLoS One, 9, e91029.

Kelleher, I., Keeley, H., Corcoran, P., Lynch, F., Fitzpatrick, C., Devlin, N., Molloy, C., Roddy, S., Clarke, M. C., Harley, M., Arseneault, L., Wasserman, C., Carli, V., Sarchiapone, M., Hoven, C., Wasserman, D. \& Cannon, M. 2012. Clinicopathological significance of psychotic experiences in non-psychotic young people: evidence from four population-based studies. Br J Psychiatry, 201, 26-32.

Kelleher, I., Corcoran, P., Keeley, H., Wigman, J. T., Devlin, N., Ramsay, H., Wasserman, C., Carli, V., Sarchiapone, M., Hoven, C., Wasserman, D. \& Cannon, M. 2013a. Psychotic symptoms and population risk for suicide attempt: a prospective cohort study. JAMA Psychiatry, 70, 940-8.

Kelleher, I., Keeley, H., Corcoran, P., Ramsay, H., Wasserman, C., Carli, V., Sarchiapone, M., Hoven, C., Wasserman, D. \& Cannon, M. 2013b. Childhood trauma and psychosis in a prospective cohort study: cause, effect, and directionality. Am J Psychiatry, 170, 734-41.

Kelleher, I. \& Devylder, J. E. 2017. Hallucinations in borderline personality disorder and common mental disorders. Br J Psychiatry, 210, 230-231.

Kendler, K. S. \& Gardner, C. O., Jr. 1998. Boundaries of major depression: an evaluation of DSM-IV criteria. Am J Psychiatry, 155, 172-7.

Kinoshita, Y., Shimodera, S., Nishida, A., Kinoshita, K., Watanabe, N., Oshima, N., Akechi, T., Sasaki, T., Inoue, S., Furukawa, T. A. \& Okazaki, Y. 2011. Psychotic-like experiences are associated with violent behavior in adolescents. Schizophr Res, 126, 245-51.

Klonsky, E. D., May, A. M. \& Saffer, B. Y. 2016. Suicide, Suicide Attempts, and Suicidal Ideation. Annu Rev Clin Psychol, 12, 307-30.

Large, M. M. \& Nielssen, O. 2011. Violence in first-episode psychosis: a systematic review and meta-analysis. Schizophr Res, 125, 209-20.

Latalova, K., Kamaradova, D. \& Prasko, J. 2014. Violent victimization of adult patients with severe mental illness: a systematic review. Neuropsychiatr Dis Treat, 10, 1925-39.

Lataster, J., Myin-Germeys, I., Lieb, R., Wittchen, H. U. \& Van Os, J. 2012. Adversity and psychosis: a 10-year prospective study investigating synergism between early and recent adversity in psychosis. Acta Psychiatr Scand, 125, 388-99.

Link, B. G., Stueve, A. \& Phelan, J. 1998. Psychotic symptoms and violent behaviors: probing the components of "threat/control-override" symptoms. Soc Psychiatry Psychiatr Epidemiol, 33 Suppl 1, S55-60.

Linscott, R. J. \& Van Os, J. 2013. An updated and conservative systematic review and meta-analysis of epidemiological evidence on psychotic experiences in children and adults: on the pathway from proneness to persistence to dimensional expression across mental disorders. Psychol Med, 43, 1133-49.

Logdberg, B., Nilsson, L. L., Levander, M. T. \& Levander, S. 2004. Schizophrenia, neighbourhood, and crime. Acta Psychiatr Scand, 110, 92-7.

Lopez-Duran, N. L., Kovacs, M. \& George, C. J. 2009. Hypothalamic-pituitary-adrenal axis dysregulation in depressed children and adolescents: a meta-analysis. Psychoneuroendocrinology, 34, 1272-83.

Lundstrom, S., Chang, Z., Rastam, M., Gillberg, C., Larsson, H., Anckarsater, H. \& Lichtenstein, P. 2012. Autism spectrum disorders and autistic like traits: similar etiology in the extreme end and the normal variation. Arch Gen Psychiatry, 69, 46-52.

Maas, C., Herrenkohl, T. I. \& Sousa, C. 2008. Review of research on child maltreatment and violence in youth. Trauma Violence Abuse, 9, 56-67.

Maj, M. 2005. "Psychiatric comorbidity": an artefact of current diagnostic systems? Br J Psychiatry, 186, 182-4.

Martin, G., Thomas, H., Andrews, T., Hasking, P. \& Scott, J. G. 2015. Psychotic experiences and psychological distress predict contemporaneous and future non-suicidal self-injury and suicide attempts in a sample of Australian school-based adolescents. Psychol Med, 45, 429-37. 
May, A. M. \& Klonsky, E. D. 2016. What Distinguishes Suicide Attempters From Suicide Ideators? A MetaAnalysis of Potential Factors. Clinical Psychology: Science and Practice, 23, 5-20.

Mcgorry, P. \& Van Os, J. 2013. Redeeming diagnosis in psychiatry: timing versus specificity. Lancet, 381, 343-5. Meehl, P. E. 1962. Schizotaxia, schizotypy, schizophrenia. American psychologist, 17, 827.

Mojtabai, R. 2006. Psychotic-like experiences and interpersonal violence in the general population. Soc Psychiatry Psychiatr Epidemiol, 41, 183-90.

Monroe, S. M. \& Simons, A. D. 1991. Diathesis-stress theories in the context of life stress research: implications for the depressive disorders. Psychol Bull, 110, 406-25.

Morgan, C., Reininghaus, U., Reichenberg, A., Frissa, S., Team, S. E. S., Hotopf, M. \& Hatch, S. L. 2014. Adversity, cannabis use and psychotic experiences: evidence of cumulative and synergistic effects. Br J Psychiatry, 204, 346-53.

Morgan, C. \& Gayer-Anderson, C. 2016. Childhood adversities and psychosis: evidence, challenges, implications. World Psychiatry, 15, 93-102.

Murray, R. M., Jones, P. B., Susser, E., Van Os, J. \& Cannon, M. 2002. The Epidemiology of Schizophrenia, Cambridge University Press.

Myin-Germeys, I., Van Os, J., Schwartz, J. E., Stone, A. A. \& Delespaul, P. A. 2001. Emotional reactivity to daily life stress in psychosis. Arch Gen Psychiatry, 58, 1137-44.

Myin-Germeys, I., Delespaul, P. \& Van Os, J. 2003. The experience sampling method in psychosis research. Current Opinion in Psychiatry, 16, S33-S38.

Myin-Germeys, I. \& Van Os, J. 2007. Stress-reactivity in psychosis: evidence for an affective pathway to psychosis. Clin Psychol Rev, 27, 409-24.

Nederlof, A. F., Muris, P. \& Hovens, J. E. 2012. Psychotic-like experiences and aggressive behavior in a nonclinical sample. Personality and Individual Differences, 53, 33-37.

Nishida, A., Sasaki, T., Nishimura, Y., Tanii, H., Hara, N., Inoue, K., Yamada, T., Takami, T., Shimodera, S., Itokawa, M., Asukai, N. \& Okazaki, Y. 2010. Psychotic-like experiences are associated with suicidal feelings and deliberate self-harm behaviors in adolescents aged 12-15 years. Acta Psychiatr Scand, 121, 301-7.

Nishida, A., Shimodera, S., Sasaki, T., Richards, M., Hatch, S. L., Yamasaki, S., Usami, S., Ando, S., Asukai, N. \& Okazaki, Y. 2014. Risk for suicidal problems in poor-help-seeking adolescents with psychotic-like experiences: findings from a cross-sectional survey of 16,131 adolescents. Schizophr Res, 159, 257-62.

Nock, M. K., Prinstein, M. J. \& Sterba, S. K. 2009. Revealing the form and function of self-injurious thoughts and behaviors: A real-time ecological assessment study among adolescents and young adults. J Abnorm Psychol, 118, 816-27.

Nolen-Hoeksema, S. \& Watkins, E. R. 2011. A Heuristic for Developing Transdiagnostic Models of Psychopathology: Explaining Multifinality and Divergent Trajectories. Perspect Psychol Sci, 6, 589-609.

Odgers, C. L., Mulvey, E. P., Skeem, J. L., Gardner, W., Lidz, C. W. \& Schubert, C. 2009. Capturing the ebb and flow of psychiatric symptoms with dynamical systems models. Am J Psychiatry, 166, 575-82.

Oram, S., Trevillion, K., Feder, G. \& Howard, L. M. 2013. Prevalence of experiences of domestic violence among psychiatric patients: systematic review. Br J Psychiatry, 202, 94-9.

Owen, P. R. 2012. Portrayals of schizophrenia by entertainment media: a content analysis of contemporary movies. Psychiatr Serv, 63, 655-9.

Palm, A., Danielsson, I., Skalkidou, A., Olofsson, N. \& Hogberg, U. 2016. Violence victimisation-a watershed for young women's mental and physical health. Eur J Public Health, 26, 861-867.

Palmier-Claus, J. E., Taylor, P. J., Gooding, P., Dunn, G. \& Lewis, S. W. 2012. Affective variability predicts suicidal ideation in individuals at ultra-high risk of developing psychosis: an experience sampling study. Br J Clin Psychol, 51, 72-83.

Pruessner, M., Cullen, A. E., Aas, M. \& Walker, E. F. 2017. The neural diathesis-stress model of schizophrenia revisited: An update on recent findings considering illness stage and neurobiological and methodological complexities. Neurosci Biobehav Rev, 73, 191-218.

Read, J., Van Os, J., Morrison, A. P. \& Ross, C. A. 2005. Childhood trauma, psychosis and schizophrenia: a literature review with theoretical and clinical implications. Acta Psychiatr Scand, 112, 330-50.

Rueve, M. E. \& Welton, R. S. 2008. Violence and mental illness. Psychiatry (Edgmont), 5, 34-48. 
Schimanski, I. D., Mouat, K. L., Billinghurst, B. L. \& Linscott, R. J. 2017. Preliminary evidence that schizophrenia liability at age 15 predicts suicidal ideation two years later. Schizophr Res, 181, 60-62.

Selten, J. P. \& Cantor-Graae, E. 2007. Hypothesis: social defeat is a risk factor for schizophrenia? Br J Psychiatry Suppl, 51, s9-12.

Selten, J. P., Van Der Ven, E., Rutten, B. P. \& Cantor-Graae, E. 2013. The social defeat hypothesis of schizophrenia: an update. Schizophr Bull, 39, 1180-6.

Sharifi, V., Eaton, W. W., Wu, L. T., Roth, K. B., Burchett, B. M. \& Mojtabai, R. 2015. Psychotic experiences and risk of death in the general population: 24-27 year follow-up of the Epidemiologic Catchment Area study. Br J Psychiatry, 207, 30-6.

Shevlin, M., Houston, J. E., Dorahy, M. J. \& Adamson, G. 2008. Cumulative traumas and psychosis: an analysis of the national comorbidity survey and the British Psychiatric Morbidity Survey. Schizophr Bull, 34, $193-9$.

Sullivan, T. P., Khondkaryan, E., Dos Santos, N. P. \& Peters, E. N. 2011. Applying experience sampling methods to partner violence research: safety and feasibility in a 90-day study of community women. Violence Against Women, 17, 251-66.

Swanson, J. W., Borum, R., Swartz, M. S. \& Monahan, J. 1996. Psychotic symptoms and disorders and the risk of violent behaviour in the community. Criminal Behaviour and Mental Health, 6, 309-329.

Thompson, W. K., Gershon, A., O'hara, R., Bernert, R. A. \& Depp, C. A. 2014. The prediction of study-emergent suicidal ideation in bipolar disorder: a pilot study using ecological momentary assessment data. Bipolar Disord, 16, 669-77.

Tijssen, M. J., Van Os, J., Wittchen, H. U., Lieb, R., Beesdo, K., Mengelers, R., Krabbendam, L. \& Wichers, M. 2010. Evidence that bipolar disorder is the poor outcome fraction of a common developmental phenotype: an 8-year cohort study in young people. Psychol Med, 40, 289-99.

Van De Leemput, I. A., Wichers, M., Cramer, A. O., Borsboom, D., Tuerlinckx, F., Kuppens, P., Van Nes, E. H., Viechtbauer, W., Giltay, E. J., Aggen, S. H., Derom, C., Jacobs, N., Kendler, K. S., Van Der Maas, H. L., Neale, M. C., Peeters, F., Thiery, E., Zachar, P. \& Scheffer, M. 2014. Critical slowing down as early warning for the onset and termination of depression. Proc Natl Acad Sci U S A, 111, 87-92.

Van Nierop, M., Van Os, J., Gunther, N., Myin-Germeys, I., De Graaf, R., Ten Have, M., Van Dorsselaer, S., Bak, M. \& Van Winkel, R. 2012. Phenotypically continuous with clinical psychosis, discontinuous in need for care: evidence for an extended psychosis phenotype. Schizophr Bull, 38, 231-8.

Van Nierop, M., Viechtbauer, W., Gunther, N., Van Zelst, C., De Graaf, R., Ten Have, M., Van Dorsselaer, S., Bak, M., Genetic, R., Investigators, O. U. O. P. \& Van Winkel, R. 2015. Childhood trauma is associated with a specific admixture of affective, anxiety, and psychosis symptoms cutting across traditional diagnostic boundaries. Psychol Med, 45, 1277-88.

Van Os, J., Hanssen, M., Bijl, R. \& Ravelli, A. 2000. Straus (1969) revisited: A psychosis continuum in the general population? Schizophrenia Research, 45, 11-20.

Van Os, J., Pedersen, C. B. \& Mortensen, P. B. 2004. Confirmation of synergy between urbanicity and familial liability in the causation of psychosis. Am J Psychiatry, 161, 2312-4.

Van Os, J. \& Linscott, R. J. 2012. Introduction: The extended psychosis phenotype--relationship with schizophrenia and with ultrahigh risk status for psychosis. Schizophr Bull, 38, 227-30.

Van Os, J. 2013. The dynamics of subthreshold psychopathology: implications for diagnosis and treatment. Am J Psychiatry, 170, 695-8.

Van Os, J., Tan, N. \& Honings, S. 2015. Functionele diagnostiek in de forensische psychiatrie: een literatuuronderzoek., Maastricht, Maastricht University.

Van Os, J. \& Reininghaus, U. 2016. Psychosis as a transdiagnostic and extended phenotype in the general population. World Psychiatry, 15, 118-24.

Van Rossum, I., Dominguez, M. D., Lieb, R., Wittchen, H. U. \& Van Os, J. 2011. Affective dysregulation and reality distortion: a 10-year prospective study of their association and clinical relevance. Schizophr Bull, 37, 561-71.

Van Winkel, R., Stefanis, N. C. \& Myin-Germeys, I. 2008. Psychosocial stress and psychosis. A review of the neurobiological mechanisms and the evidence for gene-stress interaction. Schizophr Bull, 34, 1095-105. 


\section{Chapter 6}

Van Zelst, C. 2009. Stigmatization as an environmental risk in schizophrenia: a user perspective. Schizophr Bull, 35, 293-6.

Walker, E., Mittal, V. \& Tessner, K. 2008. Stress and the hypothalamic pituitary adrenal axis in the developmental course of schizophrenia. Annu Rev Clin Psychol, 4, 189-216.

Werbeloff, N., Dohrenwend, B. P., Yoffe, R., Van Os, J., Davidson, M. \& Weiser, M. 2015. The association between negative symptoms, psychotic experiences and later schizophrenia: a population-based longitudinal study. PLoS One, 10, e0119852.

Wichers, M., Groot, P. C. \& Psychosystems, E. S. M. G. E. W. S. G. 2016. Critical Slowing Down as a Personalized Early Warning Signal for Depression. Psychother Psychosom, 85, 114-6.

Wigman, J. T., Van Nierop, M., Vollebergh, W. A., Lieb, R., Beesdo-Baum, K., Wittchen, H. U. \& Van Os, J. 2012. Evidence that psychotic symptoms are prevalent in disorders of anxiety and depression, impacting on illness onset, risk, and severity--implications for diagnosis and ultra-high risk research. Schizophr Bull, 38, 247-57.

Wigman, J. T., Van Os, J., Abidi, L., Huibers, M. J., Roelofs, J., Arntz, A., Kelleher, I. \& Peeters, F. P. 2014. Subclinical psychotic experiences and bipolar spectrum features in depression: association with outcome of psychotherapy. Psychol Med, 44, 325-36.

Yu, R., Geddes, J. R. \& Fazel, S. 2012. Personality disorders, violence, and antisocial behavior: a systematic review and meta-regression analysis. J Pers Disord, 26, 775-92. 
Valorisation 
In this thesis, the longitudinal associations between psychosis and multidirectional violence were examined in the general population. More specifically, the aim of the thesis was to gain insight into the associations between psychotic experiences on the one hand, and self-directed violence, interpersonal violence perpetration and interpersonal violence victimisation on the other. Many previous studies examined the associations between full-blown psychotic disorders and multidirectional violence, showing that individuals with psychotic disorders are at increased risk of interpersonal violence perpetration (Fazel et al. 2009), violence victimisation (de Mooij et al. 2015) and selfdirected violence (Palmer et al. 2005). However, evidence has shown that psychotic experiences are not only prevalent in individuals with psychotic disorder, but also in general population individuals with non-psychotic mental disorders and in individuals without any mental disorders (Linscott and van Os 2013; Kelleher et al. 2012). Moreover, psychotic experiences are phenotypically and aetiologically continuous with fullblown psychotic disorder and represent the softest behavioural expression of distributed population risk of psychosis (Lataster et al. 2009; van Nierop et al. 2012; van Os and Reininghaus 2016). Therefore, subclinical psychotic experiences can be used to gain insight into the underlying mechanisms of the frequently reported association between psychotic disorders and violence. Increased insight into psychosis and violence is required, since the societal and economic impact of both phenomena is large.

\section{Relevance}

\section{Scientific relevance:}

The studies presented in this thesis provided evidence that individuals with psychotic experiences are at increased risk of self-directed violence, interpersonal violence perpetration and interpersonal violence victimisation. Thus, the present thesis contributed to the scientific knowledge on the associations between psychotic experiences and multidirectional violence. The results showed that the associations between psychotic experiences and multidirectional violence were analogous to the associations between fullblown psychosis and multidirectional violence. Therefore, the often reported increased risk of multidirectional violence in individuals with psychotic disorders can be extrapolated to subjects with less severe symptoms in the general population. This finding confirms that psychotic experiences are part of the extended psychosis phenotype and thus share demographic, pathophysiological and aetiological correlates with full-blown psychotic disorder. Because psychotic experiences are more prevalent than full-blown psychotic disorder, i.e. 7.2\% (Linscott and van Os 2013) versus 3.1\% (Perala et al. 2007), the epidemiological and statistical possibilities to study psychosis and multidirectional violence in future studies can be increased by using psychotic experiences in general population samples. 


\section{Socioeconomic relevance}

The socioeconomic impact of violence is tremendous. In the United States, the national costs of suicide and suicide attempts were \$58.4 billion in 2013 (Shepard et al. 2016), while the costs associated with interpersonal violence were even higher (Waters et al. 2005). In addition, research has shown that victims of violence are at increased risk of various mental disorders, suicide and other health problems (Krug et al. 2002). Therefore, in order to decrease the socioeconomic burden of violence, it is important to gain insight into the risk factors associated with violence. Schizophrenia and other psychotic disorders have previously been associated with an increased risk of interpersonal violence (Fazel et al. 2009) and suicidal behaviour (Palmer et al. 2005). The studies presented in this thesis identified psychotic experiences as a risk factor for self-directed violence (Honings et al. 2016a; Honings et al. 2016c), interpersonal violence perpetration (Honings et al. 2016b) and interpersonal violence victimisation as well (Honings et al. 2017). Therefore, the incidence of multidirectional violence might be reduced by adequate treatment of psychotic experiences, for example by early intervention programs for psychosis. These early detection programs for psychotic disorders have been developed in the past decades (McGorry et al. 2007) and are proven to be costeffective (Mihalopoulos et al. 2009). Based on the results presented in this study, it is likely that these programs will also decrease the incidence of multidirectional violence, even though psychotic experiences are of course only one of many risk factors on the pathway towards violence.

The socioeconomic burden related to schizophrenia and other psychotic disorders is enormous as well (World Health Organization 1998; Andlin-Sobocki and Rossler 2005). In order to reduce the incidence of psychosis, and thus reduce the socioeconomic impact of psychosis, insight into risk factors for psychosis is required. Many risk factors for psychosis have been identified, including cannabis use (Moore et al. 2007), urbanization (Marcelis et al. 1998) and childhood trauma (Morgan and Gayer-Anderson 2016). The present thesis identified adult victimisation as a new risk factor for psychosis, and showed that adult victimisation, childhood victimisation and psychosis were interrelated throughout the life course. Thus, the incidence of psychosis could be reduced by interventions aimed at reducing victimisation.

\section{Target groups}

\section{Patients and their relatives}

The results of this thesis are of interest to both patients and their relatives. For patients with non-psychotic mental disorders, such as depression or anxiety disorders, the results of this thesis are particularly important. The results of chapter 3 showed that indi- 
viduals with non-psychotic mental disorders who experienced psychotic experiences, were more likely to display suicidal attempts than individuals with non-psychotic mental disorders in absence of psychotic experiences (Honings et al. 2016c). Similarly, individuals with hallucinations in the context of a non-psychotic mental disorder were more likely to perpetrate interpersonal violence than individuals without psychotic experiences (Honings et al. 2016b). Therefore, individuals with non-psychotic mental disorders and their relatives should receive psychoeducation including information on psychotic experiences and the risk of self-directed and interpersonal violence perpetration. Thereby, patients or their relatives can seek professional help early, thus decreasing the risk of self-directed or interpersonal violence.

\section{Health care professionals}

The results of this thesis underline the importance of knowledge of the extended psychosis phenotype in health care professionals. Even though the first studies about the continuous expression of psychosis were published several decades ago (van Os et al. 1999; Strauss 1969), the understanding of this concept is still limited in health care professionals. Clinical practice shows that health care professionals often consider patients to be either 'psychotic' or 'non-psychotic'. However, evidence shows that this dichotomous view of psychosis is incorrect and that subclinical psychotic experiences are prevalent in the general population (Linscott and van Os 2013; van Os and Reininghaus 2016). The presence of these psychotic experiences in general population individuals has been associated with various poor mental health outcomes. The present thesis showed that individuals with psychotic experiences were at increased risk of selfdirected and interpersonal violence perpetration. This increased risk of multidirectional violence perpetration was particularly present in individuals with non-psychotic mental disorders, in which psychotic experiences serve as an indicator of severity of mental distress. Therefore, a thorough assessment of these psychotic experiences should be included in every mental state examination in order to identify individuals at risk of multidirectional violence perpetration early and offer adequate support. In the studies published in this thesis, a list of twenty psychotic symptoms was used to identify individuals with psychotic experiences. This list could be incorporated into clinical practice and serve as a guideline for the assessment of psychotic experiences. Furthermore, the results presented in chapter 5 (Honings et al. 2017) showed that psychotic experiences and victimisation were intricately interconnected throughout the life course, resulting in a complex interplay in which psychotic experiences and victimisation were bidirectionally associated. Thus, prevention strategies against victimisation, both in childhood and adulthood, are needed in order to prevent individuals from entering a malignant spiral leading to mental illness and re-victimisation. The results of this chapter can be used to increase mental health care professionals' knowledge of psychosis and victimisation, leading to an increased awareness of this complex interplay. 
Society

Stigmas about mental illness are highly prevalent in society (Corrigan and Watson 2002). In popular media, for instance, individuals with psychotic disorder are often depicted as violent (Owen 2012). Similarly, a previous study showed that $70 \%$ of adult respondents rated people with schizophrenia as dangerous and unpredictable (Crisp et al. 2000). Stigma in individuals with mental illnesses is associated with various poor outcomes, including lower self-esteem, depressive symptoms and lower quality of life (van Zelst 2009). Thus, interventions to reduce public stigma associated with mental disorders are needed. The study presented in chapter 4 of this thesis reported that individuals with psychotic experiences were more likely to display interpersonal violence perpetration than individuals without these experiences (Honings et al. 2016b). This finding might increase the public stigma associated with psychosis at first sight. However, psychotic experiences are only one of many risk factors on the route to violence and the proportion of violence attributable to individuals with psychosis is small (Walsh et al. 2002). Moreover, the thesis reported that individuals with psychotic experiences were at risk of violent victimisation as well (Honings et al. 2017). This finding is consistent with previous research that showed that individuals with schizophrenia were 14 times more likely to be victims of violence than perpetrators (Brekke et al. 2001). Therefore, the results of this thesis could also contribute to a reduction of stigma associated with psychosis, since they showed that the often reported increased risk of violence perpetration in individuals with schizophrenia can be traced back to the general population. Thus, the common stereotype that individuals with schizophrenia are dangerous should be dismissed, since this increased risk of violence is present in a large part of the general population as well. In conclusion, the results of this thesis are relevant for society at large, since they increase the knowledge of society about the complex associations between psychosis and multidirectional violence, hopefully resulting in a decrease of negative stereotypes and stigmatization of individuals with severe mental disorders.

\section{Summary and conclusion}

The results presented in this thesis have several implications, both for science, clinical mental health care as well as for society at large. On a scientific level, this thesis presented evidence that individuals with psychotic experiences are at increased risk of selfdirected violence, interpersonal violence perpetration and interpersonal violence victimisation. These findings are important for mental health care professionals, since they show that even subclinical psychotic experiences are clinically relevant. Thus, the results underline the importance of screening for psychotic experiences in all patients presenting to mental health care. By incorporating an extensive screening for psychotic experi- 
ences into every mental state examination, individuals at risk of multidirectional violence can be identified and treated early, leading to a decrease of violence associated with mental disorders. This is necessary, since the socioeconomic impact of multidirectional violence is large. Furthermore, the public stigma associated with psychotic disorders could be reduced by informing the general public about the extended psychosis phenotype and its association with multidirectional violence. 


\section{References}

Andlin-Sobocki, P. \& Rossler, W. 2005. Cost of psychotic disorders in Europe. Eur J Neurol, 12 Suppl 1, 74-7.

Brekke, J. S., Prindle, C., Bae, S. W. \& Long, J. D. 2001. Risks for individuals with schizophrenia who are living in the community. Psychiatr Serv, 52, 1358-66.

Corrigan, P. W. \& Watson, A. C. 2002. Understanding the impact of stigma on people with mental illness. World Psychiatry, 1, 16-20.

Crisp, A. H., Gelder, M. G., Rix, S., Meltzer, H. I. \& Rowlands, O. J. 2000. Stigmatisation of people with mental illnesses. Br J Psychiatry, 177, 4-7.

De Mooij, L. D., Kikkert, M., Lommerse, N. M., Peen, J., Meijwaard, S. C., Theunissen, J., Duurkoop, P. W., Goudriaan, A. E., Van, H. L., Beekman, A. T. \& Dekker, J. J. 2015. Victimisation in adults with severe mental illness: prevalence and risk factors. Br J Psychiatry, 207, 515-22.

Fazel, S., Gulati, G., Linsell, L., Geddes, J. R. \& Grann, M. 2009. Schizophrenia and violence: systematic review and meta-analysis. PLoS Med, 6, e1000120.

Honings, S., Drukker, M., Groen, R. \& Van Os, J. 2016a. Psychotic experiences and risk of self-injurious behaviour in the general population: a systematic review and meta-analysis. Psychol Med, 46, 237-51.

Honings, S., Drukker, M., Ten Have, M., De Graaf, R., Van Dorsselaer, S. \& Van Os, J. 2016b. Psychotic Experiences and Risk of Violence Perpetration and Arrest in the General Population: A Prospective Study. PLoS One, 11, e0159023.

Honings, S., Drukker, M., Van Nierop, M., Van Winkel, R., Wittchen, H. U., Lieb, R., Ten Have, M., De Graaf, R., Van Dorsselaer, S. \& Van Os, J. 2016c. Psychotic experiences and incident suicidal ideation and behaviour: Disentangling the longitudinal associations from connected psychopathology. Psychiatry Res, 245, 267275.

Honings, S., Drukker, M., Ten Have, M., De Graaf, R., Van Dorsselaer, S. \& Van Os, J. 2017. The interplay of psychosis and victimisation across the life course: a prospective study in the general population. Soc Psychiatry Psychiatr Epidemiol.

Kelleher, I., Keeley, H., Corcoran, P., Lynch, F., Fitzpatrick, C., Devlin, N., Molloy, C., Roddy, S., Clarke, M. C., Harley, M., Arseneault, L., Wasserman, C., Carli, V., Sarchiapone, M., Hoven, C., Wasserman, D. \& Cannon, M. 2012. Clinicopathological significance of psychotic experiences in non-psychotic young people: evidence from four population-based studies. Br J Psychiatry, 201, 26-32.

Krug, E. G., Mercy, J. A., Dahlberg, L. L. \& Zwi, A. B. 2002. The world report on violence and health. Lancet, 360, 1083-8.

Lataster, T., Myin-Germeys, I., Derom, C., Thiery, E. \& Van Os, J. 2009. Evidence that self-reported psychotic experiences represent the transitory developmental expression of genetic liability to psychosis in the general population. Am J Med Genet B Neuropsychiatr Genet, 150B, 1078-84.

Linscott, R. J. \& Van Os, J. 2013. An updated and conservative systematic review and meta-analysis of epidemiological evidence on psychotic experiences in children and adults: on the pathway from proneness to persistence to dimensional expression across mental disorders. Psychol Med, 43, 1133-49.

Marcelis, M., Navarro-Mateu, F., Murray, R., Selten, J. P. \& Van Os, J. 1998. Urbanization and psychosis: a study of 1942-1978 birth cohorts in The Netherlands. Psychol Med, 28, 871-9.

Mcgorry, P. D., Killackey, E. \& Yung, A. R. 2007. Early intervention in psychotic disorders: detection and treatment of the first episode and the critical early stages. Med J Aust, 187, S8-10.

Mihalopoulos, C., Harris, M., Henry, L., Harrigan, S. \& Mcgorry, P. 2009. Is early intervention in psychosis costeffective over the long term? Schizophr Bull, 35, 909-18.

Moore, T. H., Zammit, S., Lingford-Hughes, A., Barnes, T. R., Jones, P. B., Burke, M. \& Lewis, G. 2007. Cannabis use and risk of psychotic or affective mental health outcomes: a systematic review. Lancet, 370, 319-28.

Morgan, C. \& Gayer-Anderson, C. 2016. Childhood adversities and psychosis: evidence, challenges, implications. World Psychiatry, 15, 93-102.

Owen, P. R. 2012. Portrayals of schizophrenia by entertainment media: a content analysis of contemporary movies. Psychiatr Serv, 63, 655-9. 
Palmer, B. A., Pankratz, V. S. \& Bostwick, J. M. 2005. The lifetime risk of suicide in schizophrenia: a reexamination. Arch Gen Psychiatry, 62, 247-53.

Perala, J., Suvisaari, J., Saarni, S. I., Kuoppasalmi, K., Isometsa, E., Pirkola, S., Partonen, T., Tuulio-Henriksson, A., Hintikka, J., Kieseppa, T., Harkanen, T., Koskinen, S. \& Lonnqvist, J. 2007. Lifetime prevalence of psychotic and bipolar I disorders in a general population. Arch Gen Psychiatry, 64, 19-28.

Shepard, D. S., Gurewich, D., Lwin, A. K., Reed, G. A., Jr. \& Silverman, M. M. 2016. Suicide and Suicidal Attempts in the United States: Costs and Policy Implications. Suicide Life Threat Behav, 46, 352-62.

Strauss, J. S. 1969. Hallucinations and delusions as points on continua function. Rating scale evidence. Arch Gen Psychiatry, 21, 581-6.

Van Nierop, M., Van Os, J., Gunther, N., Myin-Germeys, I., De Graaf, R., Ten Have, M., Van Dorsselaer, S., Bak, M. \& Van Winkel, R. 2012. Phenotypically continuous with clinical psychosis, discontinuous in need for care: evidence for an extended psychosis phenotype. Schizophr Bull, 38, 231-8.

Van Os, J., Verdoux, H., Maurice-Tison, S., Gay, B., Liraud, F., Salamon, R. \& Bourgeois, M. 1999. Self-reported psychosis-like symptoms and the continuum of psychosis. Soc Psychiatry Psychiatr Epidemiol, 34, 459-63.

Van Os, J. \& Reininghaus, U. 2016. Psychosis as a transdiagnostic and extended phenotype in the general population. World Psychiatry, 15, 118-24.

Van Zelst, C. 2009. Stigmatization as an environmental risk in schizophrenia: a user perspective. Schizophr Bull, 35, 293-6.

Walsh, E., Buchanan, A. \& Fahy, T. 2002. Violence and schizophrenia: examining the evidence. Br J Psychiatry, 180, 490-5.

Waters, H. R., Hyder, A. A., Rajkotia, Y., Basu, S. \& Butchart, A. 2005. The costs of interpersonal violence--an international review. Health Policy, 73, 303-15.

World Health Organization 1998. Schizophrenia and public health, Geneva, World Health Organization. 

Summary 
Psychosis and violence are both prevalent in the general population and are often associated with each other, both in popular media and in scientific literature. Different types of violence exist, and violence can be either self-directed or directed towards others. In other words, violence is a multidirectional phenomenon. In the past decades, studies have shown that individuals with schizophrenia and other psychotic disorders are at increased risk of violence perpetration, both self-directed violence (i.e. suicidal behaviour and non-suicidal self-injurious behaviour) and interpersonal violence. In addition, studies have shown that violence victimisation is prevalent among individuals with psychotic disorder. Thus, psychotic disorders are associated with an increased risk of multidirectional violence. However, studies have shown that psychotic experiences, such as hallucinations and delusions, are also prevalent in individuals without schizophrenia or any other psychotic disorder. The presence of these psychotic experiences has been associated with an increased risk of various poor mental health outcomes, including both psychotic and non-psychotic mental disorders. However, it remains unclear whether individuals with psychotic experiences are at increased risk of multidirectional violence as well. The aim of this thesis was to gain insight into this question.

Chapter 1 contains a general introduction, introducing the two main concepts of this thesis, i.e. psychosis and violence. In addition, an overview of the literature on the associations between psychosis and violence is given. The aims and outline of the thesis are described as well.

In chapter 2, a systematic review and meta-analysis on the association between psychotic experiences and self-directed violence in the general population is presented, summarizing and integrating all available evidence on this association. A systematic review and meta-analysis was required, since the published studies showed marked variation. Therefore, a systematic literature search was performed, identifying twentyfive studies. Results of the systematic review and meta-analysis showed that the risk of self-directed violence was three times higher in individuals with psychotic experiences compared to those without. However, the results showed that the reported association between psychotic experiences and self-directed violence was confounded by the presence of depression symptoms. Depression is prevalent among individuals with psychotic experiences and is associated with an increased risk of self-directed violence. In conclusion, the results presented in chapter 2 showed that individuals with psychotic experiences were at increased risk of self-directed violence. However, it remained unclear to what degree the association between psychotic experiences and self-directed violence was specific. Therefore, more research was required to separate psychotic experiences from connected non-psychotic psychopathology and to determine any specific association with self-directed violence.

In chapter 3, the association between psychotic experiences and self-directed violence was examined, while attempting to disentangle the longitudinal association from connected non-psychotic psychopathology. Data from three prospective, general population cohorts (i.e. EDSP, NEMESIS and NEMESIS-2) were combined into one dataset, in- 
cluding 15387 individuals. Results showed that the presence of psychotic experiences increased the risk of incident suicidal thoughts and suicidal behaviour. Further examination of this association in subsamples stratified by presence or absence of any nonpsychotic mental disorder, revealed that there was no association between psychotic experiences and suicidal thoughts or suicidal behaviour in the subgroup without any mental disorder. However, in this subgroup, the risk of suicidal thoughts increased as PE co-occurred with other symptom domains, for instance depression or anxiety symptoms. In the subgroup with any non-psychotic mental disorder, the presence of psychotic experiences increased the risk of suicide attempts, even after adjustment for depression, mania and anxiety symptoms. In chapter 2 , it was hypothesized that the reported association between psychotic experiences and self-directed violence was non-specific, but reflected the increased risk of this outcome in distressed participants with comorbid mental disorders or high levels of psychopathology. The results of chapter 3 support this hypothesis.

In chapter 4, the association between psychotic experiences and interpersonal violence perpetration was examined using data from NEMESIS-2: a prospective cohort of 6646 general population adults with a follow-up of six years. Results showed that the presence of psychotic experiences increased the risk of incident physical violence perpetration and incident psychological violence perpetration. In addition, individuals with psychotic experiences were at increased risk of incident arrest. However, adjustment for dimensional measures of psychopathology reduced all association considerably. After adjustment, hallucinations remained significantly associated with physical violence perpetration. Delusions were associated with an increased risk of arrest. There was no significant association between psychotic experiences and psychological violence perpetration in the fully adjusted model. Thus, the study showed that psychotic experiences may differentially predict physical violence perpetration and arrest. However, it remained unclear why hallucinations were predictive of physical violence perpetration, while delusions were predictive of arrest.

In chapter 5, the association between psychotic experiences and violence victimisation was examined in the general population. Previous studies showed that childhood victimisation increased the risk of psychotic experiences. In addition, studies have shown that adult victimisation is prevalent among individuals with severe mental disorders, for instance schizophrenia. However, it was unclear whether psychosis increased the risk of incident adult victimisation, or vice versa. Moreover, a moderating effect of childhood victimisation on the association between psychotic experiences and adult victimisation had not been investigated. The aim of the study presented in chapter 5 was to investigate these research questions using data from NEMESIS-2. Results showed that individuals with psychotic experiences were at increased risk of incident adult victimisation. Individuals with childhood victimisation were at increased risk of adult victimisation as well. However, this was through competing pathways, as suggested by a negative interaction between psychotic experiences and childhood victimisa- 
tion. In addition, results showed that adult victimisation and childhood victimisation both independently increased the risk of psychotic experiences. However, there was no interaction between adult victimisation and childhood victimisation. In conclusion, the study showed that psychosis and victimisation are interconnected, resulting in a complex interplay between psychosis and victimisation throughout the life course.

In chapter 6, the results presented in the previous chapters are summarized, discussed and integrated. In addition, strengths and weaknesses of the studies presented in this thesis are discussed, providing suggestions for future research. Finally, the implications of the results of this thesis are highlighted. 

Samenvatting 

Psychose en geweld zijn beide veel voorkomende fenomenen, die zowel in wetenschappelijke publicaties als in populaire media vaak met elkaar in verband worden gebracht. Geweld kan zowel gericht zijn tegen de eigen persoon als tegen anderen. Met andere woorden, geweld is een multidirectioneel fenomeen. In de afgelopen jaren hebben diverse onderzoeken aangetoond dat mensen met schizofrenie en andere psychotische stoornissen een verhoogd risico hebben om geweld te vertonen, zowel jegens zichzelf (suïcidale gedragingen en automutilatie) als jegens anderen (interpersoonlijk geweld). Daarnaast is er ook bewijs dat mensen met psychotische stoornissen vaker slachtoffer zijn van geweld. Kortom, psychotische stoornissen zijn geassocieerd met een verhoogd risico op multidirectioneel geweld. Wetenschappelijk onderzoek heeft echter aangetoond dat psychotische verschijnselen, zoals wanen en hallucinaties, niet alleen voorkomen bij personen met psychotische stoornissen zoals schizofrenie, maar ook bij mensen zonder deze stoornissen. Mensen met psychotische verschijnselen in afwezigheid van een psychotische stoornis hebben wel een verhoogd risico om een psychotische stoornis te ontwikkelen. Daarnaast hebben ze ook een verhoogd risico op andere psychiatrische aandoeningen. Het is echter onbekend of mensen met deze psychotische verschijnselen ook een verhoogd risico hebben op multidirectioneel geweld. In dit proefschrift wordt deze vraagstelling onderzocht.

Hoofdstuk 1 bevat een algemene inleiding waarin de twee hoofdthema's van dit proefschrift, te weten psychose en geweld, worden geïntroduceerd en beschreven. Daarnaast wordt er ook een overzicht van de beschikbare literatuur over psychose en geweld gepresenteerd en wordt er een overzicht gegeven van de opbouw en doelstellingen van het proefschrift.

In hoofdstuk 2 wordt een systematische review en meta-analyse beschreven waarin de beschikbare literatuur over de associatie tussen psychotische verschijnselen en zelfgericht geweld in de algemene populatie wordt samengevat en geïntegreerd. De reden om dit literatuuronderzoek uit te voeren was het feit dat er in de literatuur uiteenlopende resultaten werden gepubliceerd over deze associatie. Er werd daarom een systematisch literatuuronderzoek verricht, waarbij er vijfentwintig bruikbare studies werden gevonden en geïncludeerd. De resultaten van deze systematische review en metaanalyse tonen aan dat personen met psychotische verschijnselen ruim driekeer zoveel kans hebben op zelfgericht geweld als personen zonder psychotische verschijnselen. $\mathrm{Er}$ zijn echter sterke aanwijzingen dat de associatie tussen psychotische verschijnselen en zelfgericht geweld gedeeltelijk verklaard kan worden door de aanwezigheid van depressieve symptomen. Depressieve klachten komen namelijk frequent voor bij personen met psychotische verschijnselen en vergroten het risico op zelfgericht geweld. Samenvattend tonen de resultaten van hoofdstuk 2 aan dat aanwezigheid van psychotische verschijnselen het risico op zelfgericht geweld weliswaar vergroot, maar dat er meer onderzoek noodzakelijk is om inzicht te krijgen in de mate waarin dit verhoogd risico verklaard kan worden door gelijktijdig optredende non-psychotische psychopathologie. 
In hoofdstuk 3 wordt dezelfde vraagstelling onderzocht als in hoofdstuk 2, waarbij er wordt getracht om meer duidelijkheid te verkrijgen over de rol van non-psychotische psychopathologie op de longitudinale associatie tussen psychotische verschijnselen en zelfgericht geweld. Er wordt hiervoor gebruik gemaakt van drie verschillende databases, namelijk de EDSP, NEMESIS en NEMESIS-2. In totaal werden er 15387 personen geïncludeerd in het onderzoek. De resultaten tonen aan dat personen met psychotische verschijnselen tijdens de baselinemeting een verhoogd risico hebben op suïcidale gedachten en suïcidepogingen tijdens een van de follow-upmetingen. Wanneer dezelfde analyse echter wordt uitgevoerd in een subgroep van mensen zonder een nonpsychotische psychiatrische stoornis, blijkt dat er geen associatie meer bestaat tussen psychotische verschijnselen en suïcidaliteit. Het risico op suïcidale gedachten neemt binnen deze subgroep wel toe wanneer psychotische verschijnselen optreden met andere psychiatrische symptomen, bijvoorbeeld angst of depressie. Binnen de subgroep van mensen die wel een non-psychotische psychiatrische stoornis hebben, vergroot de aanwezigheid van psychotische verschijnselen het risico op suïcidepogingen wel aanzienlijk. De in hoofdstuk 2 voorgestelde hypothese dat de associatie tussen psychotische verschijnselen en zelfgericht geweld non-specifiek is, maar wordt veroorzaakt door het gelijktijdig optreden van non-psychotische psychopathologie, wordt door de resultaten van hoofdstuk 3 bevestigd.

In hoofdstuk 4 wordt de associatie tussen psychotische verschijnselen en interpersoonlijke geweldspleging onderzocht. Er werd hiervoor gebruikt gemaakt van de NEMESIS-2 database: een prospectieve studie waarin 6646 personen uit de algemene bevolking werden geïncludeerd en opgevolgd gedurende zes jaren. De resultaten toonden aan dat personen met psychotische verschijnselen tijdens de baselinemeting een verhoogd risico hadden op fysieke en psychologische geweldspleging tijdens één van de follow-upmetingen. Daarnaast hadden deze personen ook een verhoogd risico om gearresteerd te worden. Correctie voor de aanwezigheid van dimensionele nonpsychotische psychopathologie zorgde ervoor dat de associaties minder sterk werden. Desalniettemin behielden personen met hallucinaties een verhoogd risico op het vertonen van fysiek geweld. De aanwezigheid van wanen vergrootte het risico op fysieke geweldspleging niet, maar vergrootte wel de kans op arrestatie door de politie. Er werd geen verband gevonden tussen psychotische verschijnselen en psychologische geweldspleging. Het is onduidelijk waarom wanen en hallucinaties verschillend geassocieerd zijn met de diverse uitkomstmaten van deze studie.

Hoofdstuk 5 beschrijft een studie waarin de associatie tussen psychotische verschijnselen en slachtofferschap van geweld werd onderzocht in de algemene populatie. Eerder onderzoek had reeds aangetoond dat trauma's tijdens de kinderleeftijd het risico op psychotische verschijnselen vergrootte. Daarnaast was het bekend dat slachtofferschap van geweld tijdens het volwassen leven vaak voorkomt bij personen met ernstige psychiatrische stoornissen zoals bijvoorbeeld schizofrenie. Het was echter onbekend of het slachtoffer zijn van geweld het risico om psychotische verschijnselen te ontwikkelen 
vergrootte, of dat personen met psychotische verschijnselen meer risico lopen om slachtoffer te worden van geweld. Om deze vraagstelling te onderzoeken werd er opnieuw gebruik gemaakt van de NEMESIS-2 database. De resultaten van het onderzoek toonden aan dat de aanwezigheid van psychotische verschijnselen tijdens de baselinemeting het risico op slachtofferschap tijdens de follow-upmeting vergrootte. De aanwezigheid van trauma's in de kindertijd vergrootte het risico op slachtofferschap op volwassenleeftijd ook. Er was echter geen positieve interactie tussen psychotische verschijnselen en trauma's in de kinderleeftijd voor de uitkomstmaat slachtofferschap tijdens de volwassen leeftijd. Daarnaast toonden de resultaten ook aan dat slachtofferschap van geweld tijdens de baselinemeting het risico op psychotische verschijnselen tijdens de follow-upmetingen vergrootte. Dit gold zowel voor slachtofferschap van geweld tijdens de kinderleeftijd als op volwassen leeftijd. Slachtofferschap tijdens de kindertijd en slachtofferschap op volwassen leeftijd vormden zodoende cumulatieve risicofactoren voor psychotische verschijnselen later in het leven. Samenvattend toonden de resultaten aan dat er sprake is van een bidirectionele associatie tussen psychotische verschijnselen en slachtofferschap van geweld, resulterend in een complex samenspel tussen psychose en slachtofferschap gedurende het leven.

In hoofdstuk 6 worden de resultaten van de voorgaande hoofdstukken samengevat, bediscussieerd en geïntegreerd. Tevens worden de sterkte- en zwaktepunten van de gepresenteerde studies besproken en worden er voorstellen gedaan voor toekomstig onderzoek. Tenslotte worden ook de implicaties van de studies in dit proefschrift beschreven. 

Dankwoord 

Een proefschrift ontstaat niet van vandaag op morgen. Tussen het moment waarop het eerste idee voor het schrijven van een proefschrift ontstaat en de uiteindelijke voltooiing ervan liggen vele jaren, ervaringen en ontmoetingen met mensen. Nu de eindstreep is behaald, is het mogelijk om terug te blikken op deze periode.

Terugkijkend in de tijd kan ik het moment waarop het zaadje voor het idee om een proefschrift te gaan schrijven in mijn gedachten werd geplant zeer nauwkeurig vaststellen: 5 oktober 2011. Op deze datum verdedigde mijn broer Rick namelijk zijn proefschrift aan de universiteit in Leiden. Zoals het een oudere broer betaamt, wierp hij met deze gebeurtenis de schaduw op waarin ik als jongere broer niet graag vertoefde, maar die mij wel motiveerde om net als hij een promotietraject na te streven. De kans om te starten met het onderzoek dat uiteindelijk zou resulteren in mijn promotieonderzoek ontstond echter pas in 2014, toen ik voor mijn wetenschapsstage in het kader van de geneeskundeopleiding in contact kwam met Jim van Os. Gedurende een half jaar werkten we samen aan een literatuurstudie over functionele diagnostiek in de forensische psychiatrie, en gaandeweg raakte ik meer en meer enthousiast over wetenschappelijk onderzoek. Ik ben dan ook dankbaar dat ik de kans heb gekregen om dit onderzoek voort te zetten na mijn afstuderen in de vorm van een promotietraject en wil iedereen die aan de totstandkoming van dit proefschrift heeft bijgedragen bedanken.

Prof. dr. van Os, beste Jim, in de afgelopen jaren heb je als promotor niet alleen bijgedragen aan mijn academische vorming, maar ook aan mijn vorming als toekomstig psychiater. Hoewel we slechts enkele keren samen patiënten hebben gezien op de poli in het Maastrichts Universitair Medisch Centrum, staan deze contacten mij nog helder voor de geest, omdat je vanuit je eigen enthousiasme en positiviteit altijd op zoek ging naar de kracht van de patiënt. Deze bejegening werkt niet alleen goed bij patiënten, maar ook bij promovendi. Na ieder werkoverleg was mijn enthousiasme voor het onderzoek opnieuw aangewakkerd, waardoor ik het proefschrift uiteindelijk binnen drie jaar heb kunnen voltooien. Hartelijk dank voor alles.

Dr. Drukker, beste Marjan, als copromotor heb je een belangrijke rol gespeeld in mijn onderzoek. Met eindeloos geduld heb je mij uitleg gegeven over Stata, statistiek en epidemiologie. Daarnaast was je als geen ander in staat om het enthousiasme van Jim en mij te beteugelen en in goede statistische banen te leiden. Je hebt hierdoor gezorgd voor een goed statistisch en epidemiologisch fundament waarop ik de rest van mijn wetenschappelijke carrière kan bouwen en vertrouwen. Bedankt hiervoor.

Bijzonder erkentelijk ben ik ook Margreet ten Have, Ron de Graaf en Saskia van Dorsselaer voor de NEMESIS-data en voor het actief meedenken en -schrijven als coauteurs van alle artikelen in dit proefschrift. Martine van Nierop heeft mij wegwijs gemaakt in de NEMESIS-data en heeft samen met Ruud van Winkel als co-auteur een bijdrage geleverd aan hoofdstuk 3 van dit proefschrift. Prof. dr. Wittchen en prof. dr. Lieb hebben mij in de gelegenheid gesteld om gebruik te maken van de EDSP-data. Allen bedankt voor jullie bijdragen. 
Ook de leden van de beoordelingscommissie, prof. dr. Philip Delespaul, prof. dr. Robbert-Jan Verkes, prof. dr. Jean-Paul Selten, dr. Wim Veling en dr. Ulrich Reininghaus wil ik bedanken voor het kritisch lezen en beoordelen van dit proefschrift.

Aan alle collega's van divisie 2 van de School for Mental Health and Neuroscience: jullie vormen het wetenschappelijk klimaat dat het ontstaan van dit proefschrift heeft mogelijk gemaakt. In het bijzonder dank ook aan Trees Soute, die mij op praktisch vlak voortdurend heeft ondersteund.

Special thanks to Cleon Peterson, an amazing artist who granted me permission to use his painting Glory (2014) on the book cover of this thesis.

Mijn paranimfen, Henri Boersma en Martijn Dremmen, hebben ieder op hun eigen wijze een belangrijke rol gespeeld gedurende mijn promotietraject. Henri, als mijn beste vriend heb jij gediend als klankbord, discussiepartner, moreel kompas, en nog zoveel meer. Martijn, als drummer vorm jij het kloppend hart van onze band, die mij elke zaterdagochtend de ontspanning bezorgt die onontbeerlijk was tijdens dit promotietraject. Dank voor jullie vriendschap.

Aan mijn ouders heb ik het te danken dat ik hier vandaag sta. Met hun nietaflatende steun hebben zij mij gevormd en aan hen komt daarom de meeste dank toe. 


\section{Curriculum vitae}

Steven Honings was born in Heerlen (the Netherlands) on May $21^{\text {st }}, 1990$. He attended secondary school (gymnasium) at the Bernardinus College in Heerlen, from which he graduated cum laude in 2008. Thereafter, he started studying medicine at Maastricht University. From 2009-2013, he combined his study at Maastricht University with a study at the Schumann Akademie in Utrecht, where he studied classical music theory, including musical history, analysis and counterpoint. From 2013 onwards, he did internships in various hospitals, gaining his first experience in psychiatry while working at the psychiatric wards of the Laurentius Hospital in Roermond and the Maastricht University Medical Centre. During the last year of his study, he wrote his master's thesis about functional diagnosis in forensic psychiatry together with prof. dr. Jim van Os. After his graduation in 2015, he started his PhD program at Maastricht University. At the same time, he started working as a resident in psychiatry (not in training) at the psychiatric outpatient clinic of the Maastricht University Medical Centre, where he worked with patients with somatoform disorders. As of April $1^{\text {st }} 2016$, he started his psychiatric training at the Universitaire Opleiding Psychiatrie Zuid (UOPZ), doing internships in geriatric psychiatry, addiction care and 'flexible assertive community treatment' (FACT). 



\section{List of publications}

\section{Present thesis}

Honings, S., Drukker, M., Groen, R. \& Van Os, J. 2016. Psychotic experiences and risk of self-injurious behaviour in the general population: a systematic review and metaanalysis. Psychol Med, 46, 237-51.

Honings, S., Drukker, M., Van Nierop, M., Van Winkel, R., Wittchen, H. U., Lieb, R., Ten Have, M., De Graaf, R., Van Dorsselaer, S. \& Van Os, J. 2016. Psychotic experiences and incident suicidal ideation and behaviour: Disentangling the longitudinal associations from connected psychopathology. Psychiatry Res, 245, 267-275.

Honings, S., Drukker, M., Ten Have, M., De Graaf, R., Van Dorsselaer, S. \& Van Os, J. 2016. Psychotic Experiences and Risk of Violence Perpetration and Arrest in the General Population: A Prospective Study. PLoS One, 11, e0159023.

Honings, S., Drukker, M., Ten Have, M., De Graaf, R., Van Dorsselaer, S. \& Van Os, J. 2017. The interplay of psychosis and victimisation across the life course: a prospective study in the general population. Soc Psychiatry Psychiatr Epidemiol, , 52, 1363-1374.

\section{Other}

Honings, R. \& Honings, S. 2014. Voer voor psychiaters. Willem Bilderdijk als patiënt. Tijdschrift voor Nederlandse Taal- en Letterkunde 130, 181-203.

Honings, R. \& Honings, S. 2015. The Poet as Patient. The Curious Case of Willem Bilderdijk: A Retrospective Approach. In: Grave J., Honings R. \& B., N. (eds.) Illness and Literature in the Low Countries. From the Middle Ages until the 21th Century. Göttingen: V\&R Unipress.

Van Os, J., Tan, N. \& Honings, S. 2015. Functionele diagnostiek in de forensische psychiatrie: een literatuuronderzoek., Maastricht, Maastricht University.

Ramakers, I. H., Honings, S. T., Ponds, R. W., Aalten, P., Sebastian, K., Verhey, F. R. \& Visser, P. J. 2015. The Effect of Psychological Distress and Personality Traits on Cognitive Performances and the Risk of Dementia in Patients with Mild Cognitive Impairment. J Alzheimers Dis, 46, 805-12.

Honings, S. T., Arts, B. M. \& Van Os, J. 2016. [A case-report on catatonia: a prevalent but under-recognised psychiatric illness]. Tijdschr Psychiatr, 58, 232-6. 


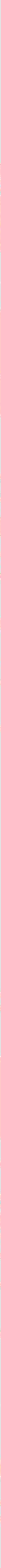

